Article

\title{
Combinatorial Synthesis of Structurally Diverse Triazole-Bridged Flavonoid Dimers and Trimers
}

\author{
Tze Han Sum ${ }^{1}$, Tze Jing Sum ${ }^{1}$, Warren R. J. D. Galloway ${ }^{1}$, Súil Collins ${ }^{1,2}$, David G. Twigg ${ }^{1}$, \\ Florian Hollfelder ${ }^{2}$ and David R. Spring ${ }^{1, *}$ \\ 1 Department of Chemistry, University of Cambridge, Lensfield Road, Cambridge CB2 1EW, UK; \\ ths29@cam.ac.uk (T.H.S.); tjs76@cam.ac.uk (T.J.S.); wrjdg2@cam.ac.uk (W.R.J.D.G.); sc806@cam.ac.uk (S.C.); \\ dgt24@cam.ac.uk (D.G.T.) \\ 2 Department of Biochemistry, University of Cambridge, Tennis Court Road, Cambridge CB2 1GA, UK; \\ fh111@cam.ac.uk \\ * Correspondence: spring@ch.cam.ac.uk; Tel.: +44-1223-336-498
}

Academic Editor: Andrea Trabocchi

Received: 29 July 2016; Accepted: 8 September 2016; Published: 16 September 2016

\begin{abstract}
Flavonoids are a large family of compounds associated with a broad range of biologically useful properties. In recent years, synthetic compounds that contain two flavonoid units linked together have attracted attention in drug discovery and development projects. Numerous flavonoid dimer systems, incorporating a range of monomers attached via different linkers, have been reported to exhibit interesting bioactivities. From a medicinal chemistry perspective, the 1,2,3-triazole ring system has been identified as a particularly attractive linker moiety in dimeric derivatives (owing to several favourable attributes including proven biological relevance and metabolic stability) and triazole-bridged flavonoid dimers possessing anticancer and antimalarial activities have recently been reported. However, there are relatively few examples of libraries of triazole-bridged flavonoid dimers and the diversity of flavonoid subunits present within these is typically limited. Thus, this compound type arguably remains underexplored within drug discovery. Herein, we report a modular strategy for the synthesis of novel and biologically interesting triazole-bridged flavonoid heterodimers and also very rare heterotrimers from readily available starting materials. Application of this strategy has enabled step-efficient and systematic access to a library of structurally diverse compounds of this sort, with a variety of monomer units belonging to six different structural subclasses of flavonoid successfully incorporated.
\end{abstract}

Keywords: flavonoid; triazole; dimer; trimer; hybridization; structural diversity

\section{Introduction}

Flavonoids are a large family of polyphenolic compounds that represent dietary constituents of importance to good health as well as a potentially important new class of pharmaceutical lead substrates [1-3]. There are several subclasses of flavonoids, including aurones, chalcones, coumarins, flavones and isoflavones, which serve as the core structural units of numerous biologically active molecules [4-7]. In recent years, synthetic compounds that contain two such flavonoid units linked together (so-called flavonoid dimers) have garnered attention from the synthetic and medicinal chemistry communities [8-17]. The generation of species that integrate two pharmacophoric entities (both homo- and hetero-dimers) is a common strategy in drug discovery [18,19] and numerous flavonoid dimer systems, incorporating a range of monomers linked in a variety of ways, have been reported to exhibit biologically useful properties [8-17]. From a medicinal chemistry perspective, the 1,2,3-triazole ring system has been identified as a particularly attractive linker moiety owing to various favourable properties including ease of synthesis, proven biological relevance and metabolic 
stability $[11,18,20]$; indeed, triazole-bridged flavonoid dimers possessing anticancer $[10,11]$ and antimalarial [11] activities have recently been reported (Figure 1). However, there are relatively few examples of libraries of triazole-bridged flavonoid dimers, and the diversity of flavonoid subunits present within these is typically limited. Thus, the triazole-bridged flavonoid dimer compound type arguably remains underexplored within drug discovery. We were interested in investigating the biological potential of triazole-bridged flavonoid dimers further and so sought access to a more structurally diverse collection of such compounds incorporating a wide range of flavonoid units. In addition, we were also interested in accessing triazole-bridged trimeric derivatives, which are also expected to have interesting biological properties. Though the synthesis of triazole-bridged flavonoid trimers has previously been reported [10], compounds of this sort are very rare and they have received relatively little attention from synthetic and medicinal chemists. Herein, we report the development of a modular strategy for the synthesis of novel triazole-bridged flavonoid heterodimers and heterotrimers. Application of this strategy has enabled concise and systematic access to a library of 46 structurally diverse compounds of this sort ( 41 dimers and five trimers) from readily available starting materials.

(a)

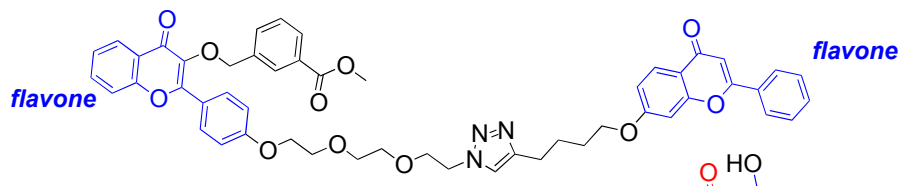

(b)

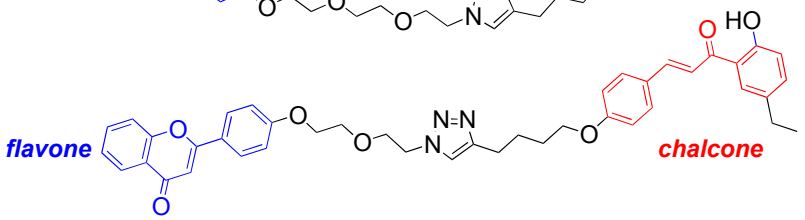

(c)

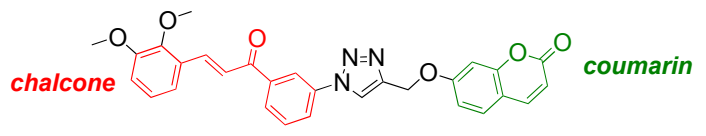

Figure 1. Three examples of biologically active triazole-linked flavonoid dimers. The flavonoid subunits in each example are highlighted in colours. (a) A modulator of multidrug resistance in some cancers [10]; (b) A modulator of multidrug resistance in some cancers [10]; (c) A compound with anticancer activity and antimalarial activity [11].

\section{Results and Discussion}

\subsection{Outline of the Synthetic Strategy}

Inspired by previous studies on the synthesis of triazole-linked flavonoid libraries $[10,11]$ we envisaged a branching-type strategy to access triazole-bridged flavonoid dimers and trimers, based around the use of iterative copper-catalysed "click"-type alkyne-azide 1,3-dipolar cycloadditions (Scheme 1) [21]. It was anticipated that flavonoid monomer units bearing a terminal alkyne group ("alkyne-flavonoid" building blocks) could be reacted with a range of flavonoid monomer units bearing a terminal azide group ("azido-flavonoid" building blocks) to furnish diverse and novel triazole-bridged flavonoid homo- and hetero-dimers (of the general structure $\mathbf{A}$, Scheme 1). The presence of a free hydroxyl functionality in either monomer unit would allow for post-cyclisation introduction of a terminal alkyne group in the dimers, thus providing the necessary synthetic handle for a further cycloaddition with varied alkyne-flavonoids to furnish structurally diverse triflavonoid derivatives of the general forms $\mathbf{B}$ and $\mathbf{C}$ (Scheme 1). The presence of additional synthetic handles in any given monomer unit should also allow for further elaboration of the dimers and trimers. Overall, it was anticipated that this modular strategy would enable step-efficient and facile access to a structurally diverse library of triazole-bridged flavonoid dimers and trimers through the use of a variety of different flavonoid building blocks belonging to different flavonoid structural subclasses. 

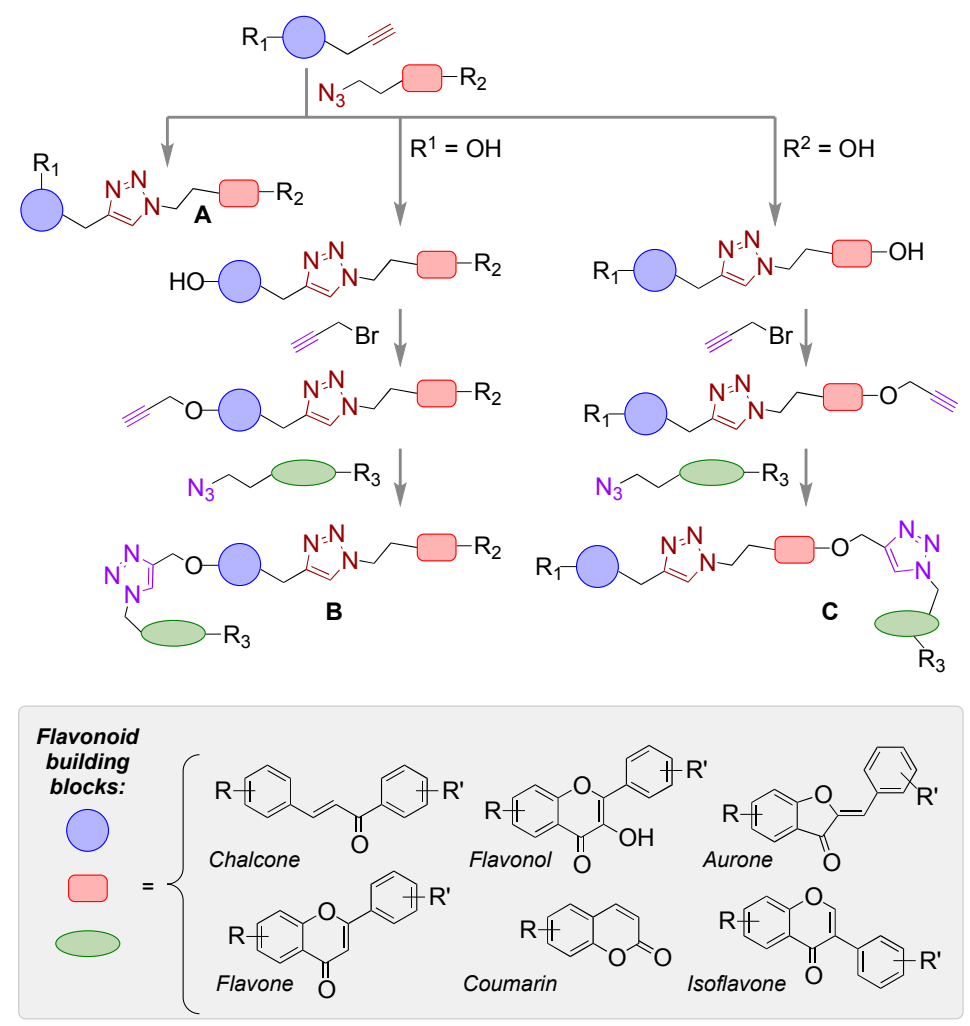

Scheme 1. Overview of the branching-type strategy towards structurally diverse triazole-bridged flavonoid heterodimers and heterotrimers. It was anticipated that homo- dimers and trimers could also be accessed through the use of appropriate building blocks.

\subsection{Building Block Design and Synthesis}

In order to facilitate the generation of structural diversity in the final compound collection, building blocks belonging to a variety of flavonoid structural subclasses (chalcone, coumarin, flavone, aurone, flavonol and isoflavone, see Scheme 1) and derivatives thereof were targeted. Structural diversity, including functional group diversity, within building blocks of some subclasses was also sought in order to further increase the overall structural diversity of the final library (as well as providing a means of introducing additional biomolecular-interacting elements into the library compounds, for example, additional bio-relevant heterocyclic motifs and hydrogen-bonding functionalities). Variation in the position of the key alkyne/azide ligation handles around the flavonoid structures was also envisaged as a strategy to further increase library structural diversity, since this would enable access to different structural isomers of any given dimers/trimers. On the basis of synthetic tractability, various alkyne-chalcones, flavones and isoflavones and azido-chalcone, flavonols and flavones were targeted. Hydroxyl-substituted building blocks were also required in order to allow access to trimeric species (as outlined in Scheme 1). Based on predicted synthetic accessibility, the syntheses of a hydroxyl-substituted alkyne-chalcone, alkyne-flavonol, azido-chalcone and azido-flavonol were targeted.

\subsubsection{Synthesis of the Alkyne-Flavonoid Building-Blocks}

\section{Alkyne-Chalcones}

Hydroxyl-substituted alkyne-chalcone 4 was accessed from phenol 1 via a two-step sequence: alkylation with propargyl bromide proceeded smoothly to yield aldehyde 2 and subsequent Claisen-Schmidt aldol reaction with acetophenone 3 yielded the target compound 4 (Scheme 2) [22]. 


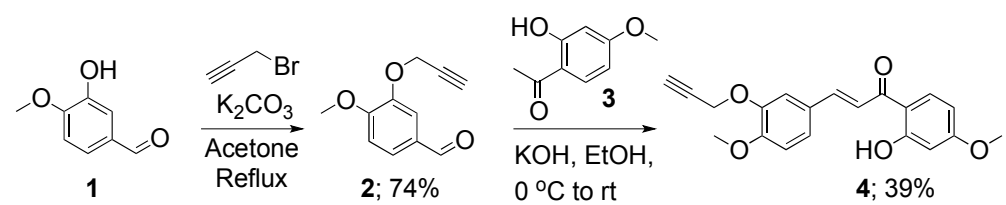

Scheme 2. Synthesis of hydroxyl-substituted alkyne-chalcone building block 4 .

Structurally diverse alkyne-chalcone building blocks 20-26, including chalconoid derivatives incorporating a range of heteroaromatic scaffolds and an unusual ferrocenyl motif, were generated from aldehydes 5-9 respectively by Claisen-Schmidt aldol condensation with various acetophenone derivatives followed by propargylation (Scheme 3).
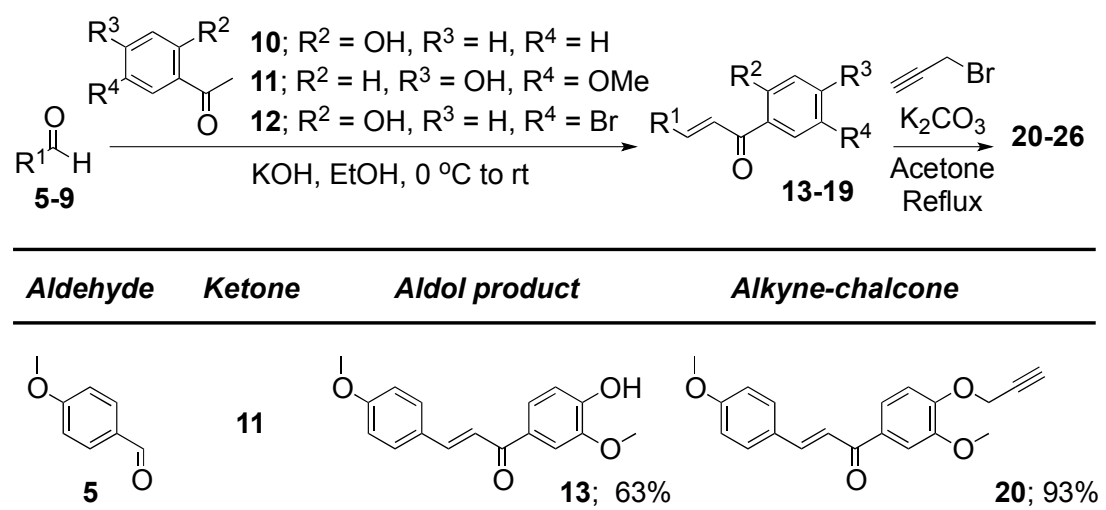<smiles></smiles>

12<smiles>Cn1cc(C=CC(=O)c2ccc(Br)c(O)c2)c2ccccc21</smiles>
10a<smiles>[3H]n1cccc1C=O</smiles><smiles>O=CC1CCCN1</smiles>
11

7<smiles>[13CH3]C1CCCO1</smiles>

12<smiles>Cn1cccc1/C=C/C(=O)c1ccccc1O</smiles><smiles>C=CCOc1ccc(Br)cc1C(=O)/C=C/c1nn(C)c2ccccc12</smiles>

21; $96 \%$<smiles>C=CCOc1ccccc1C(=O)/C=C/c1cccn1C</smiles>

23; $98 \%$<smiles>COc1cc(C(=O)/C=C/c2cccn2C)ccc1O</smiles>

16; $55 \%$<smiles>C=CCOc1ccc(/C=C/c2cccn2C)cc1OC</smiles>

22; $92 \%$<smiles>O=C(O)c1cc(Br)ccc1O</smiles><smiles>C=CCOc1ccc(Br)cc1C(=O)/C=C/c1ccco1</smiles>

24; $94 \%$

10a

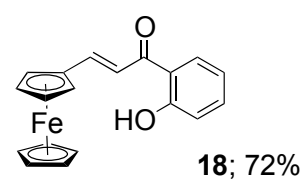<smiles>C=CCOc1ccccc1C(=O)C=CC1CC1C1CCC1</smiles>

26: $81 \%$

11

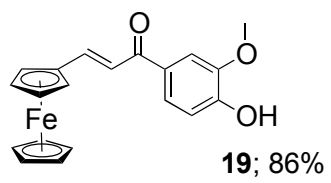

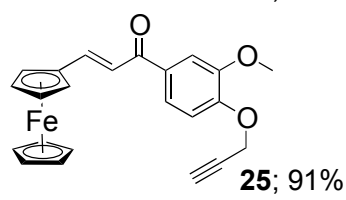

Scheme 3. Synthesis of alkyne-chalcone building blocks 20-26. 
Alkyne-Flavones

Propargylation of commercially available flavones 27-29 proceeded smoothly to furnish alkyne-flavones 30-32 with the alkyne synthetic handle appended at various positions on the flavone core unit (Scheme 4) [23,24].

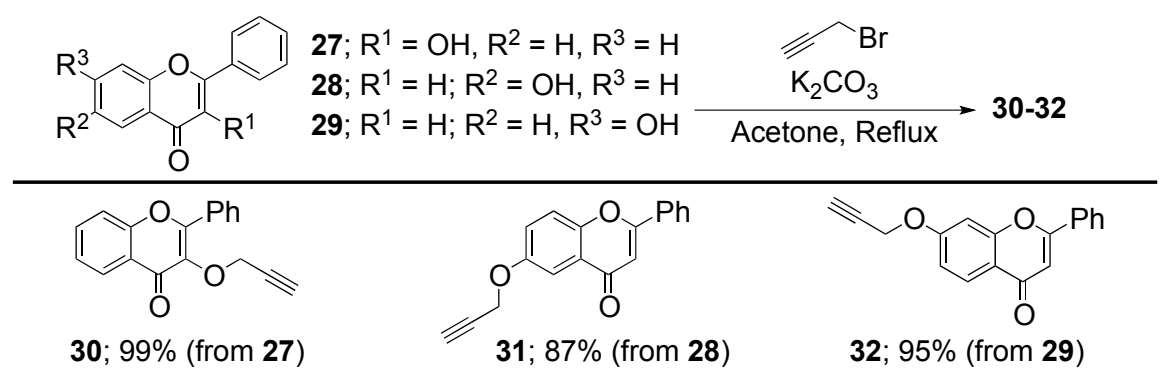

Scheme 4. Synthesis of alkyne-flavone building blocks 30-32.

Alkyne-Flavonol

The preparation of alkyne-flavonol 36 commenced with the synthesis of chalcone 35 via the Claisen-Schmidt reaction of the alkyne-substituted benzaldehyde 34 with acetophenone 10. Subsequent Algar-Flynn-Oyamada (AFO) oxidation [22] of the chalcone 35 proceeded smoothly to furnish 36 (Scheme 5).

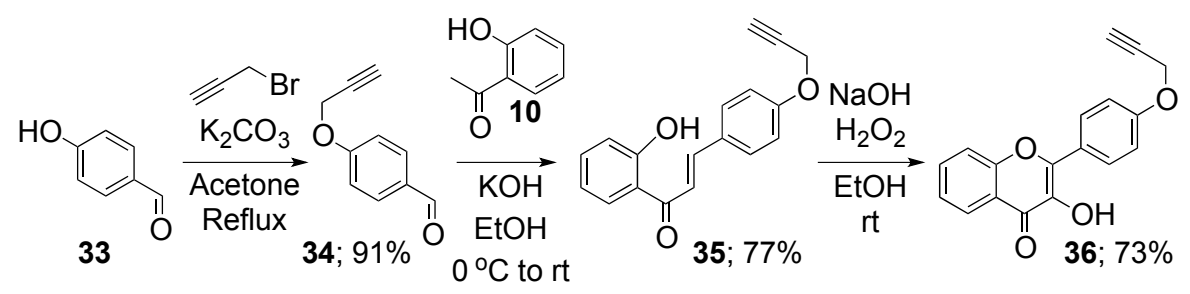

Scheme 5. Synthesis of alkyne-flavonol building block 36 .

Alkyne-Isoflavones

The preparation of the alkyne-isoflavone building blocks 45 and 46 commenced with the acylation of commercially available substituted phenols 37 and 38 with phenylacetic acids 39 and 40 to afford the deoxybenzoins 41 and 42 [25]. Subsequent cyclization of 41 and 42 in methanesulfonyl chloride afforded the corresponding isoflavones 43 and 44 which then underwent propargylation to yield the desired alkyne-isoflavones 45 and 46 (Scheme 6) [25].

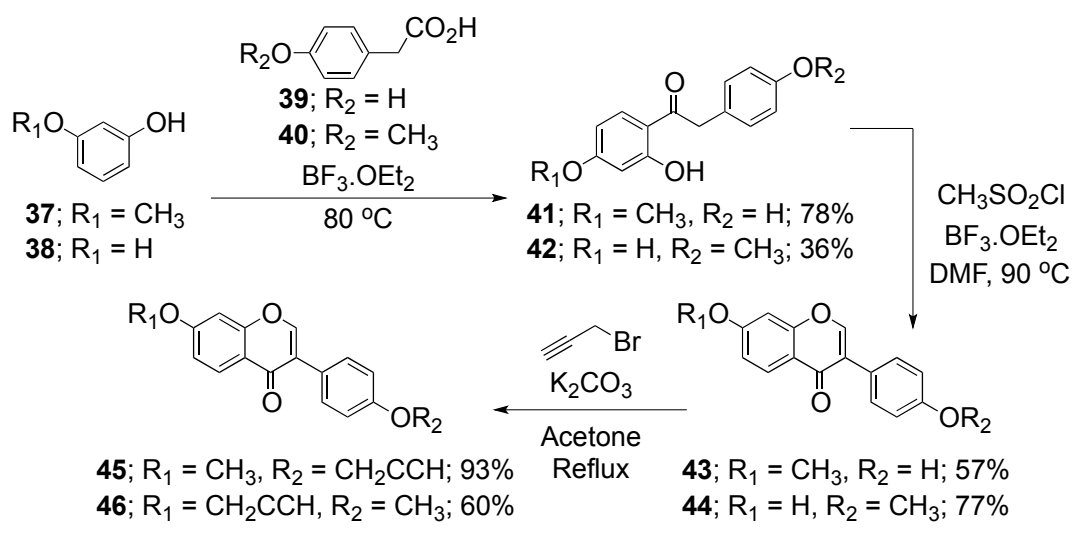

Scheme 6. Synthesis of alkyne-isoflavones 45 and 46. 
Alkyne-Coumarin

Alkyne-coumarin 48 was synthesised by propargylation of hydroxycoumarin 47 in the presence of anhydrous potassium carbonate (Scheme 7) [11].

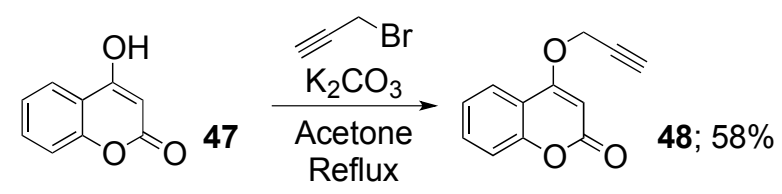

Scheme 7. Synthesis of alkyne-coumarin 48.

\section{Alkyne-Aurone}

Alkyne-aurone 56 was prepared from commercially available phloroglucinol 49 (Scheme 8). Condensation with chloroacetonitrile in the presence of $\mathrm{ZnCl}_{2}$ furnished imine 50 [26]. Subsequent hydrolysis under acidic conditions afforded ketone $\mathbf{5 1}$ which was then treated with methanolic sodium methoxide to give hydroxybenzofuranone 52 [26]. Methyl protection of the free hydroxyl groups afforded benzofuranone 53 which was then condensed with 3-hydroxybenzaldehyde 54 under basic conditions to yield hydroxyaurone 55. Subsequent propargylation gave the desired alkyne-aurone 56 in an excellent yield (Scheme 8).

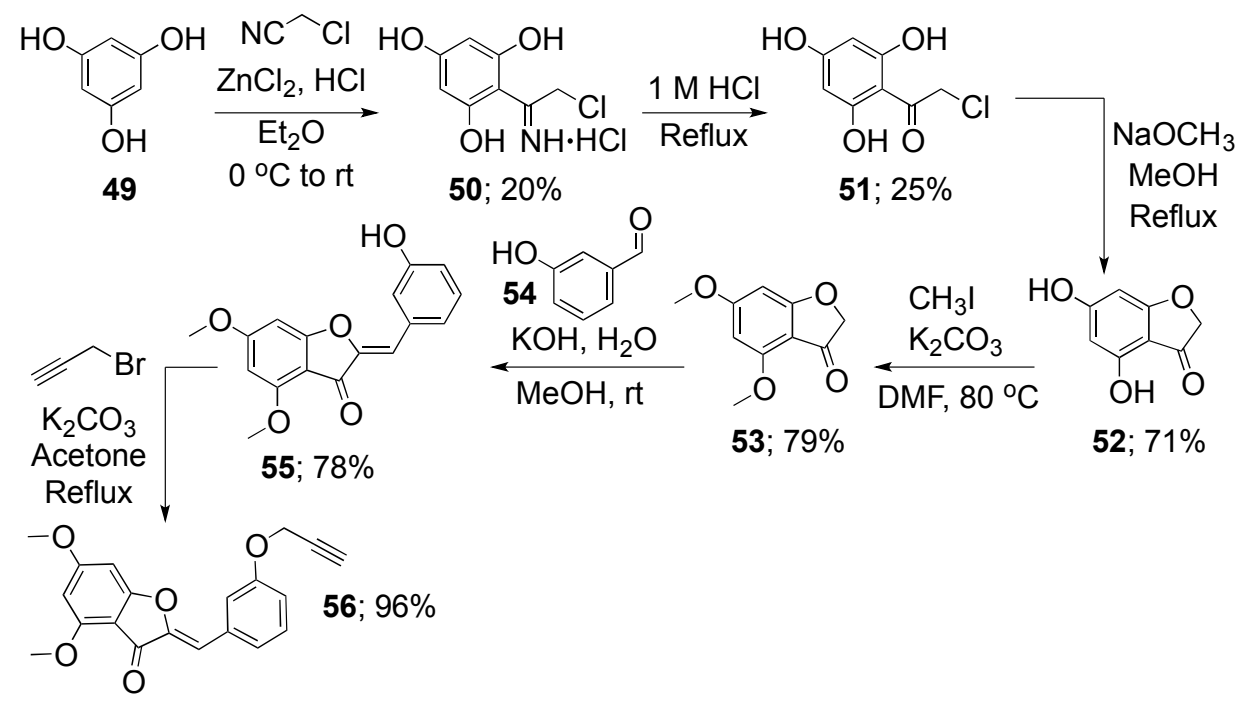

Scheme 8. Synthesis of alkyne-aurone 56.

\subsubsection{Synthesis of the Azido-Flavonoid Building Blocks}

\section{Azido-Coumarin}

Azido-coumarin 58 was prepared from readily available hydroxycoumarin 47 by alkylation (to form 57) followed by reaction with sodium azide (Scheme 9) [27].

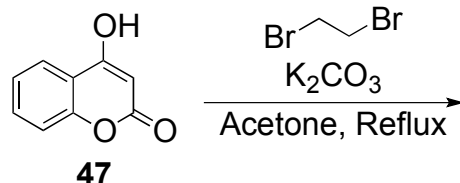

47<smiles>O=c1cc(OCCBr)c2ccccc2o1</smiles>

$57 ; 16 \%$

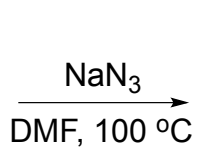<smiles>N#CCOc1cc(=O)oc2ccccc12</smiles>

58; $98 \%$

Scheme 9. Synthesis of azido-coumarin 58. 


\section{Azido-Chalcones}

Hydroxyl-substituted azido-chalcone $\mathbf{6 1}$ was prepared by a three step sequence from phenolic aldehyde 33 (Scheme 10). Reaction with 1,2-dibromoethane generated aldehyde 59 and subsequent nucleophilic substitution with sodium azide produced azide 60 in an excellent yield. Claisen-Schmidt aldol condensation with ketone 10b then yielded the target compound 61 [22]. Alternatively, Claisen-Schmidt aldol condensation of aldehydes $64-66$ and 7 with readily-prepared azido-ketone 63 furnished azido-chalcone building blocks 67-70 respectively (Scheme 11) [22].

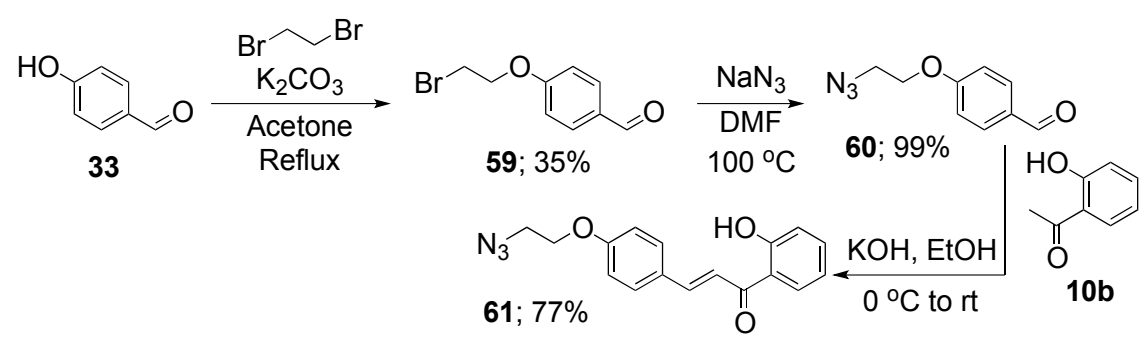

Scheme 10. Synthesis of azido-chalcone 61.

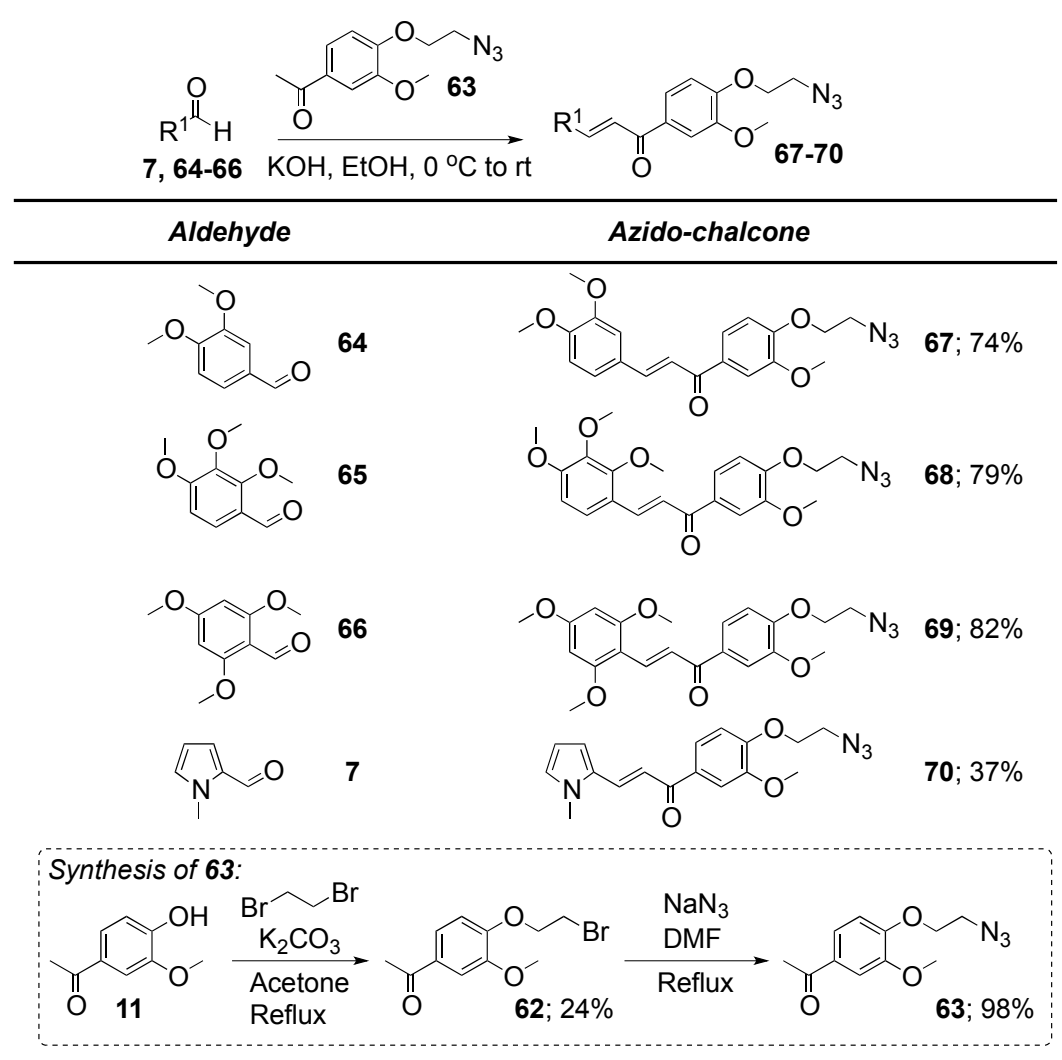

Scheme 11. Synthesis of azido-chalcones 67-70.

Azido-Flavonols

Aldehydes 59 and 72 and 73, generated by alkylation of 33, 71 and 1 respectively with 1,2-dibromoethane, were reacted with sodium azide to form $\mathbf{6 0}$ and $\mathbf{7 4}$ and 75 respectively (Scheme 12). Subsequent aldol condensation with acetophenones $\mathbf{1 0}$ or $\mathbf{3}$ (see Scheme 12) furnished chalcones $\mathbf{6 1}$ and 76-78 and AFO proceeded smoothly in all cases to furnish azido-flavonols $\mathbf{7 9}$ and 80-82 in good yields [22]. 


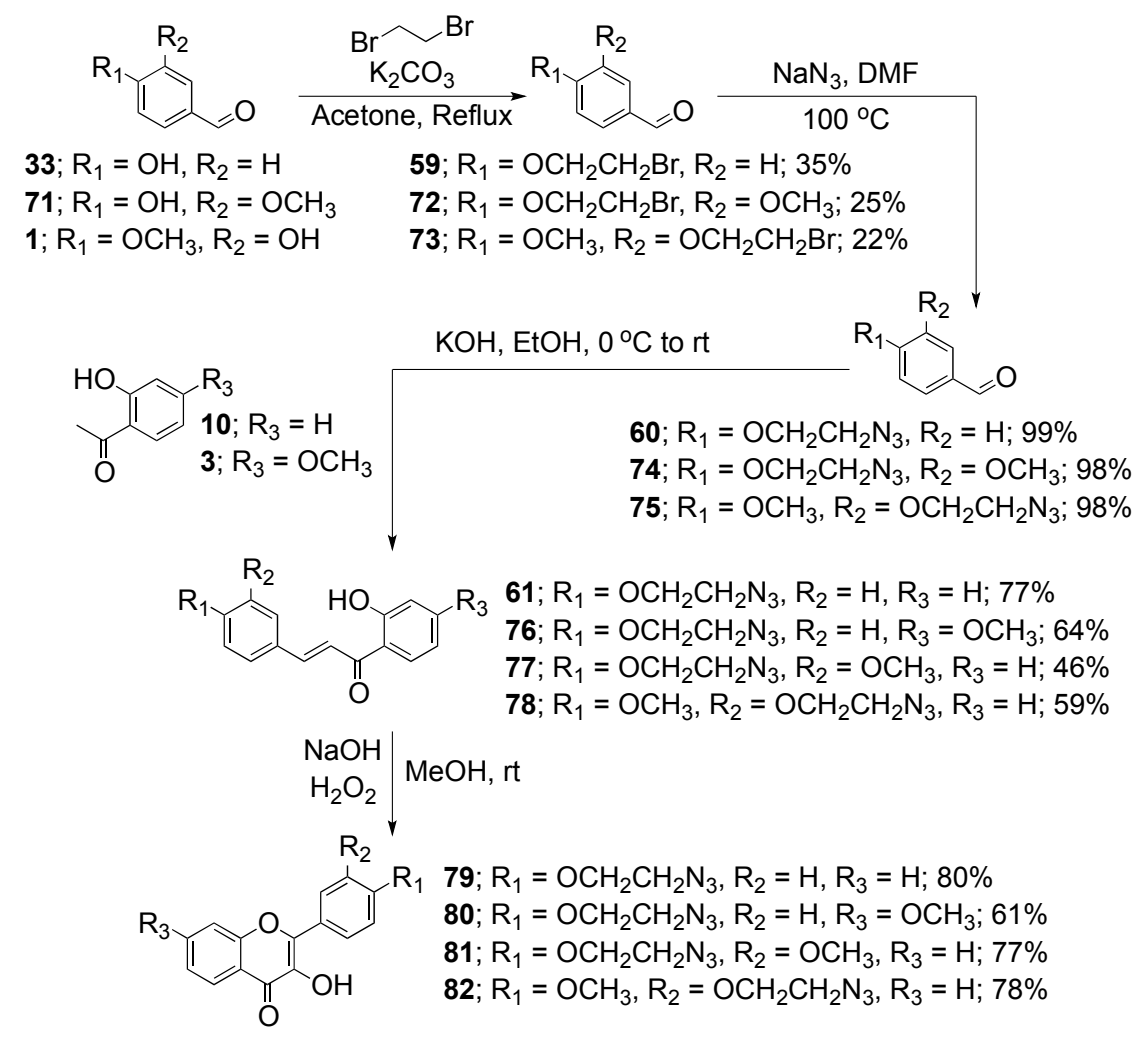

Scheme 12. Synthesis of azido-flavonols 79-82.

\section{Azido-Flavones}

Azido-flavones 85 and 86 were readily accessed from commercially available hydroxyflavones $\mathbf{2 8}$ and 29 by reaction with 1,2-dibromoethane to forge 83 and 84 followed by nucleophilic substitution with sodium azide (Scheme 13) $[10,28]$. Azido-flavone 87 was prepared in an excellent yield from chalcone 76 by an iodine-mediated oxidative cyclization (Scheme 14).<smiles>[R]Cc1cc2c(cc1[R])OCC(c1ccccc1)C2O</smiles>

28; $\mathrm{R}_{1}=\mathrm{OH}, \mathrm{R}_{2}=\mathrm{H}$ 29; $\mathrm{R}_{1}=\mathrm{H}, \mathrm{R}_{2}=\mathrm{OH}$

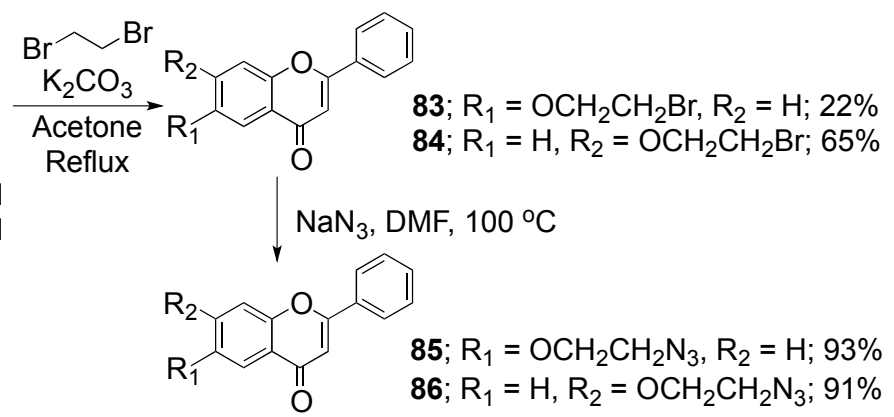

Scheme 13. Synthesis of azido-flavones 85-86.<smiles>COc1ccc(C(=O)/C=C/c2ccc(OCC#N)c(C)c2)c(O)c1</smiles>

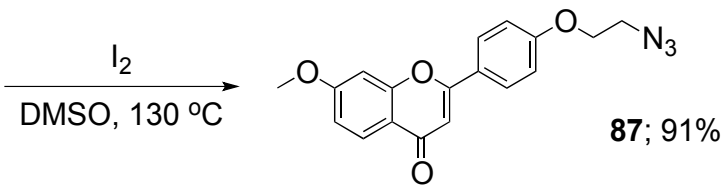

Scheme 14. Synthesis of azido-flavone 87. 
Azido-Aurones

Mercury(II) acetate-mediated oxidative cyclization of chalcones $\mathbf{6 1}$ and $\mathbf{7 6}$ furnished azido-aurones 88 and 89 respectively in excellent yields (Scheme 15) [29].
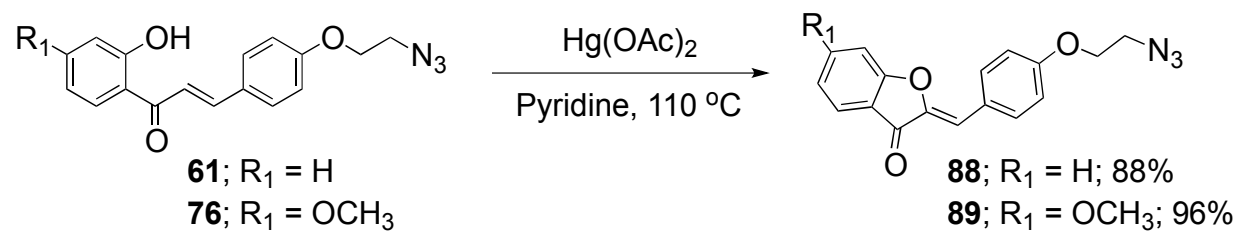

Scheme 15. Synthesis of azido-aurones 88-89.

\subsubsection{Synthesis of Triazole-Bridged Flavonoid Dimers}

With the alkyne- and azido-flavonoid building blocks in hand, we were ready to forge a series of dimeric combinations via triazole formation. Thus, various pairs of building blocks were subjected to standard copper-mediated "click" cycloaddition conditions to generate 41 distinct and diverse triazole-bridged flavonoid dimers (compounds 90-130, Schemes 16-20). The reactions generally proceeded smoothly and with high levels of regioselectivity and isolated yields of the target compounds were typically moderate-to-good. Six different biologically-relevant flavonoid structural subclasses (chalcone, flavonol, aurone, flavone, coumarin and isoflavone) were successfully incorporated into the dimer library together with other biologically-relevant features, and variation within building blocks belonging to certain subclasses allowed for the generation of additional structural diversity in the library and the concomitant introduction of additional biomolecule-interacting elements (for example, the varied heterocyclic motifs exhibited by the chalcone-chalcone dimers 90-97). Several compounds also featured groups that could provide synthetic handles for further elaboration or diversification (for example, compounds 96 and 97 and 105 and 106 contain a hydroxyl group and the aryl-bromide group present in $\mathbf{1 0 7}$ and $\mathbf{1 0 8}$ could conceivably be exploited in various metal-catalysed cross-coupling processes).

\subsubsection{Synthesis of Triazole-Bridged Flavonoid Trimers}

Propargylation of the free phenolic hydroxyl groups of triazole-bridged flavonoid dimers 126, 122, 92 and 110 and 106 led to the formation of alkyne-capped derivatives 131-135 respectively. These were successfully coupled with three azido-flavonoid building blocks (86 for 131 and 132; 87 for 133; and 89 for 134 and 135) via copper-catalysed triazole formation to furnish five structurally diverse triazole-bridged flavonoid trimers 136-140 (Scheme 21).

\subsection{Preliminary Biological Screening}

A representative sample of 13 final triazole-bridged dimers $(\mathbf{9 0}-\mathbf{9 3}, \mathbf{1 1 2}-\mathbf{1 1 4}, \mathbf{1 2 2}, \mathbf{1 2 3}, \mathbf{1 2 5}, \mathbf{1 2 6}, 129$ and 130) was screened for inhibitory activity against the aggregation of amyloid beta (1-42) $\left(A \beta_{42}\right)$, a pathological hallmark of Alzheimer's disease [30]. Aggregation of the monomeric form of the peptide into oligomeric and fibrillar species is associated with disease onset and progression. As such, the identification of compounds capable of inhibiting the aggregation process holds great potential for the development of therapeutic agents [31]. Flavonoid and chalcone derivatives have previously shown activity in perturbing the aggregation of $A \beta$, with compounds such as EGCG myricetin and morin displaying inhibitory activity in a variety of biophysical and in vivo tests [32-35]. It has also been shown that dimeric flavonoids can display enhanced inhibitory activity than their monomeric counterparts [36], suggesting that the libraries synthesised may be effective at targeting this peptide aggregation pathway. The ability of the triazole-linked dimers to inhibit the $A \beta_{42}$ aggregation was assessed using a thioflavin T (THT) assay (Figure 2). Three of the compounds screened were found 
to have moderate inhibitory activity, with $\mathbf{9 2}$ found to be the most potent and comparable to the inhibitor morin.

It is difficult to draw any firm conclusions at this time regarding structure-activity relationships in the triazole-bridged dimer compound class due to the relatively small sample size and some issues with the solubility and fluorescence behaviour of some compounds under the assay conditions. Nevertheless, this preliminary screen has identified structurally novel $\mathrm{A} \beta_{42}$ aggregation inhibitors which could represent interesting scaffolds for further study in this regard.

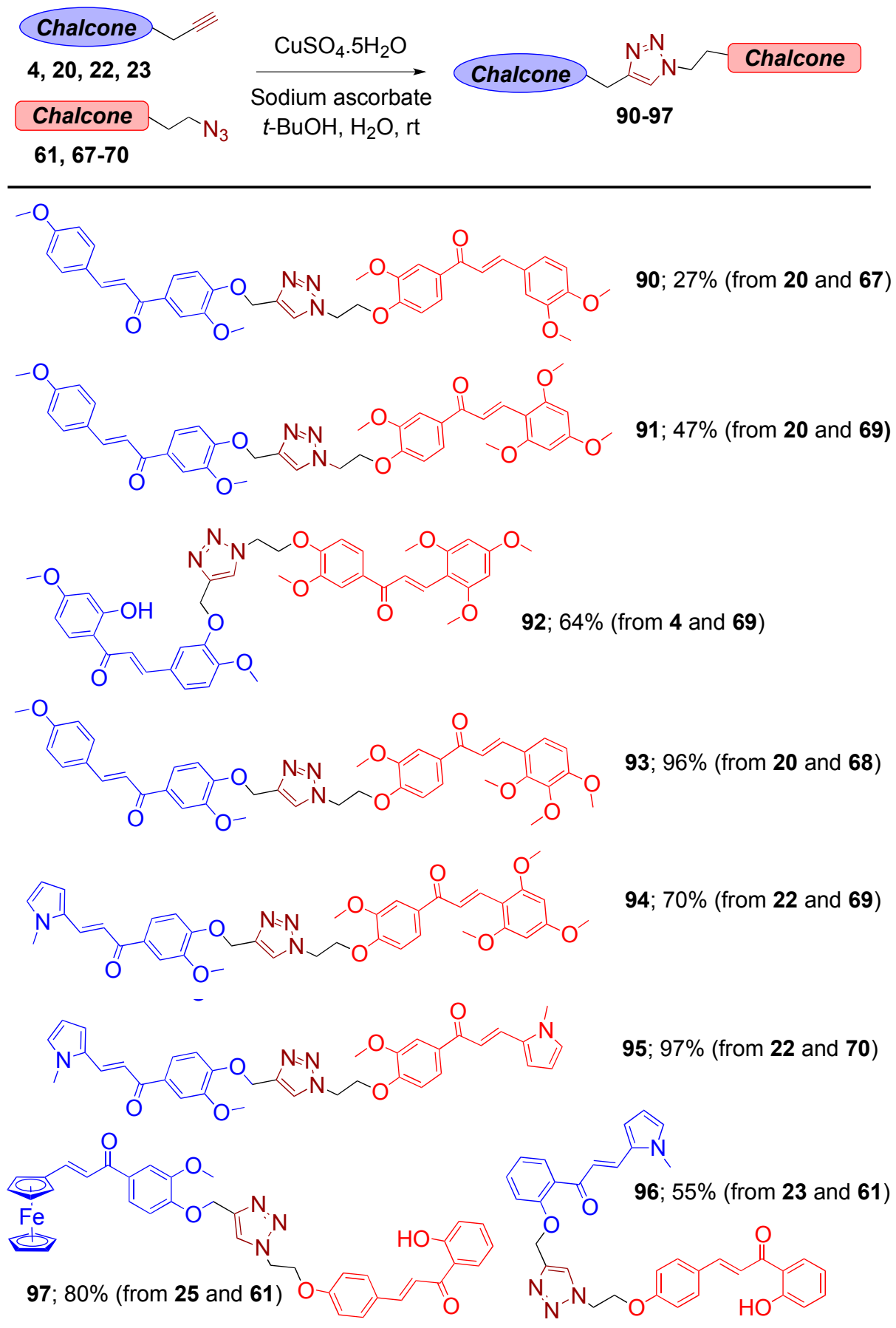

Scheme 16. Synthesis of triazole-bridged chalcone-chalcone dimers. 

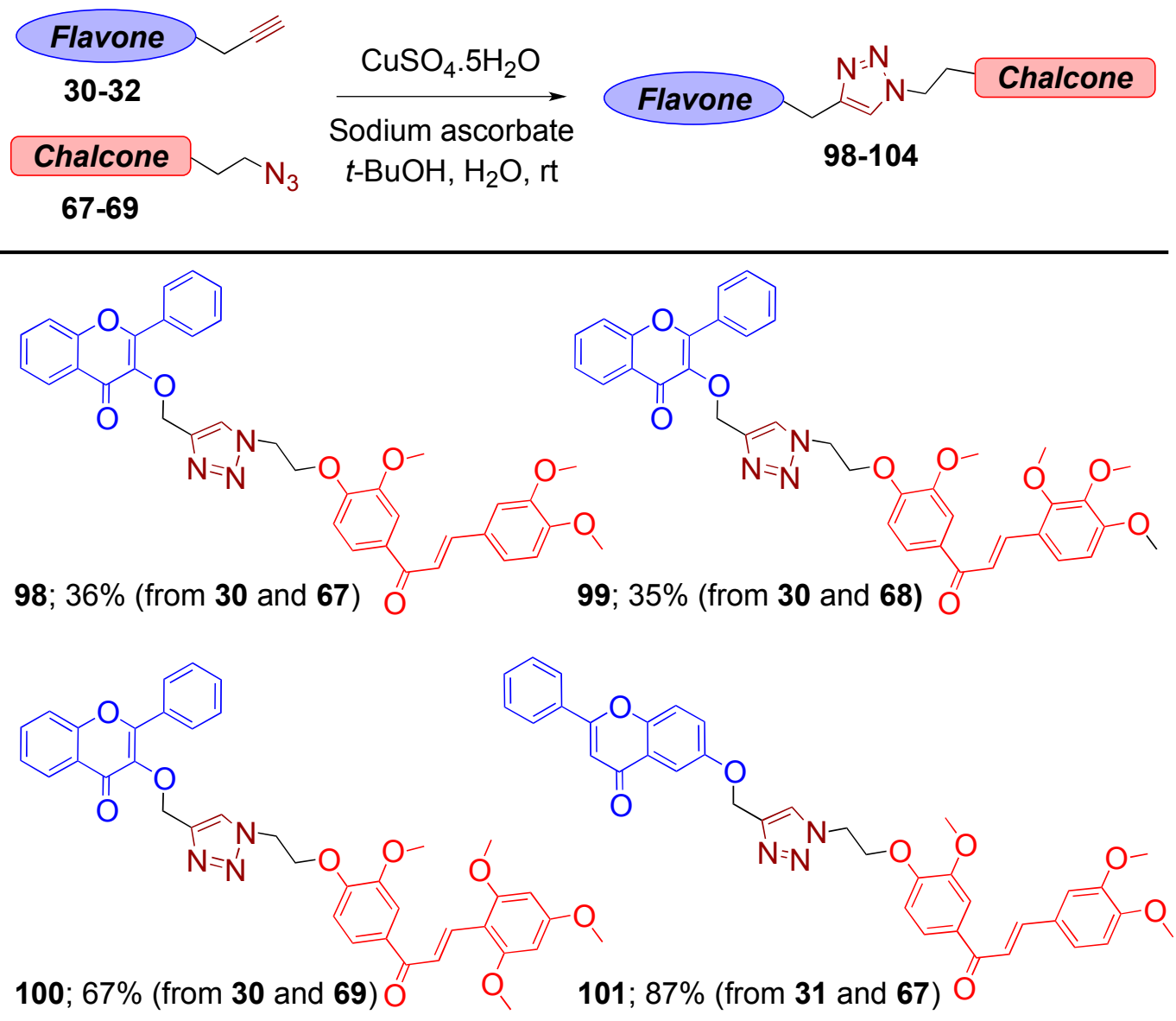

102; 75\% (from 31 and 69)<smiles>COc1cc(C(=O)/C=C/c2ccc(OC)c(OC)c2OC)ccc1OCCn1cc(COc2ccc3oc(-c4ccccc4)cc(=O)c3c2)nn1</smiles><smiles>COc1cc(OC)c(OC)cc1/C=C/C(=O)c1ccc(OCCn2cc(COc3ccc4c(c3)OC(c3ccccc3)CC4=O)nn2)c(OC)c1</smiles>

Scheme 17. Synthesis of triazole-bridged flavone-chalcone dimers. 


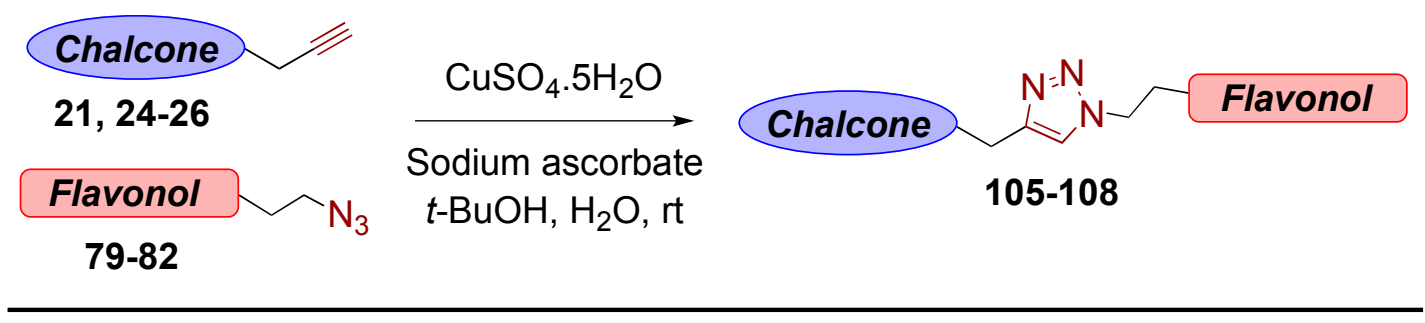<smiles>COc1ccc2c(=O)c(O)c(-c3ccc(OCCn4cc(COc5ccccc5C(=O)/C=C/c5ccccc5)nn4)cc3)oc2c1</smiles><smiles>COc1cc(C(=O)/C=C/C2CC2C2CC2)ccc1OCc1cn(CCOc2ccc(-c3oc4ccccc4c(=O)c3O)cc2)nn1</smiles>

$106 ; 38 \%$ (from 25 and 79 )<smiles>COc1ccc(-c2oc3ccccc3c(=O)c2O)cc1OCCn1cc(COc2ccc(Br)cc2C(=O)/C=C/c2cn(C)c3ccccc23)nn1</smiles>
$107 ; 40 \%$ (from 21 and 82 )<smiles>COc1cc(-c2oc3ccccc3c(=O)c2O)ccc1OCCn1cc(COc2ccc(Br)cc2C(=O)/C=C/c2ccco2)nn1</smiles>

Scheme 18. Synthesis of triazole-bridged chalcone-flavonol dimers. 

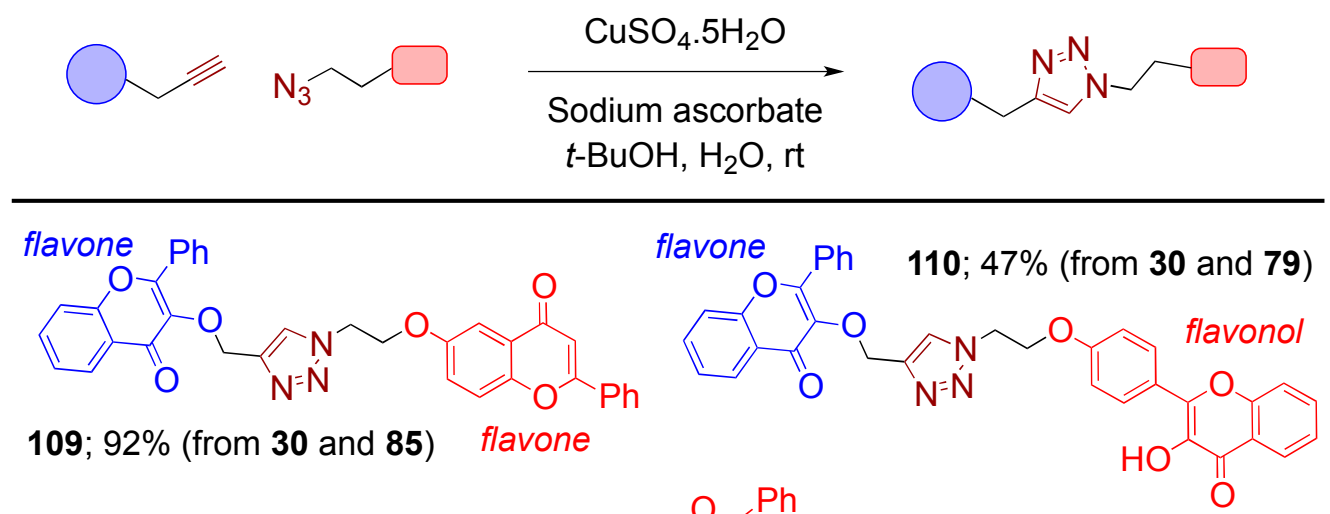

109; 92\% (from 30 and 85) flavone<smiles>COc1ccc(-c2coc3cc(OCc4cn(CCOc5ccc6oc(-c7ccccc7)cc(=O)c6c5)nn4)ccc3c2=O)cc1</smiles>

112; 95\% (from 46 and 85)<smiles></smiles>

111; 14\% (from 36 and 79)<smiles>CCOCc1cn(CCOc2ccc(/C=C/C(=O)c3ccccc3O)cc2)nn1</smiles>
coumarin 126; 78\% (from 48 and 61)

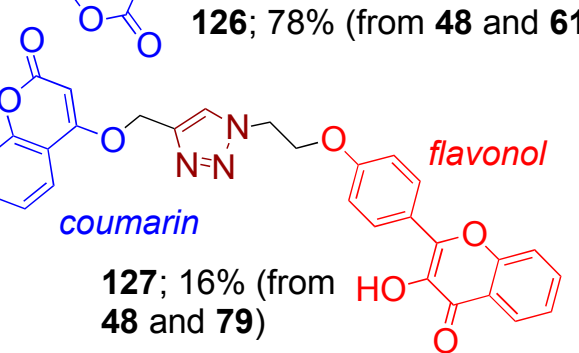<smiles></smiles>

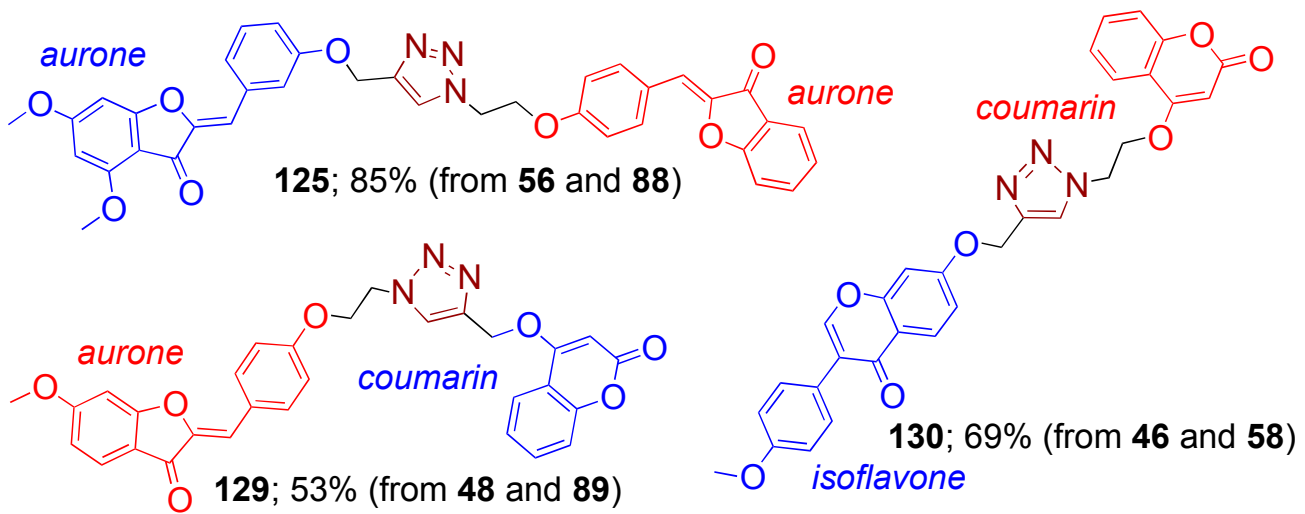<smiles>COc1ccc(-c2coc3cc(OCc4cn(CCOc5ccc(/C=C6\Oc7cc(OC)ccc7C6=O)cc5)nn4)ccc3c2=O)cc1</smiles>

124; $55 \%$ (from 46 and 89)

Scheme 19. Synthesis of some triazole-bridged dimers. 

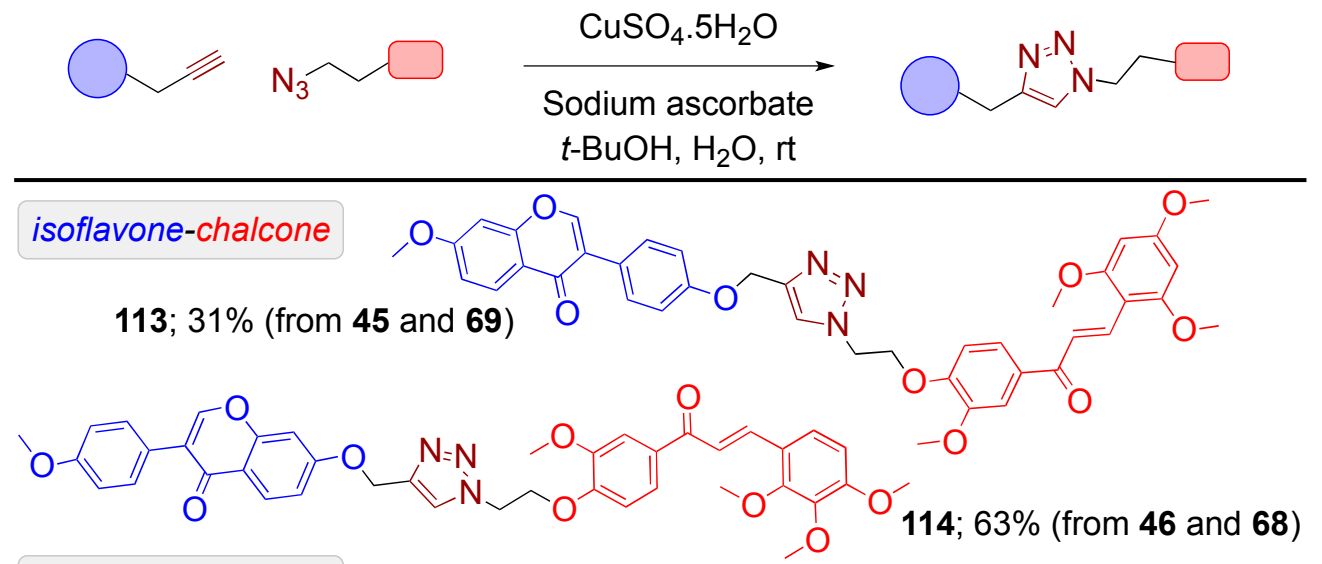<smiles>COc1ccc(/C=C/C(=O)c2ccc(OCc3cn(CCOc4ccc(/C=C5\Oc6ccccc6C5=O)cc4)nn3)c([O-])c2)cc1</smiles><smiles></smiles>

118; $48 \%$ (22 and 89)<smiles>Cn1cc(/C=C/C(=O)c2cc(Br)ccc2OCc2cnn[nH]2)c2ccccc21</smiles>

119; $21 \%$ (21 and 88 )

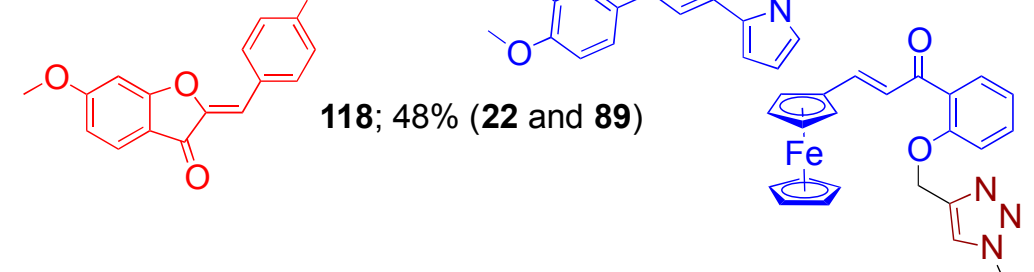

117; $78 \%$ (from 26 and 88 )<smiles>O=c1cc(-c2ccccc2)oc2ccc(OCc3cn(CCO)nn3)cc12</smiles>

121; $49 \%$ (from 31 and 89 )

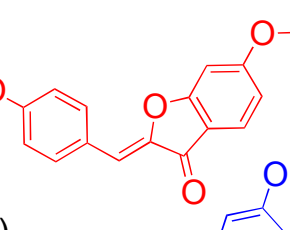

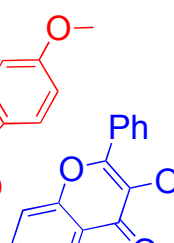<smiles></smiles>

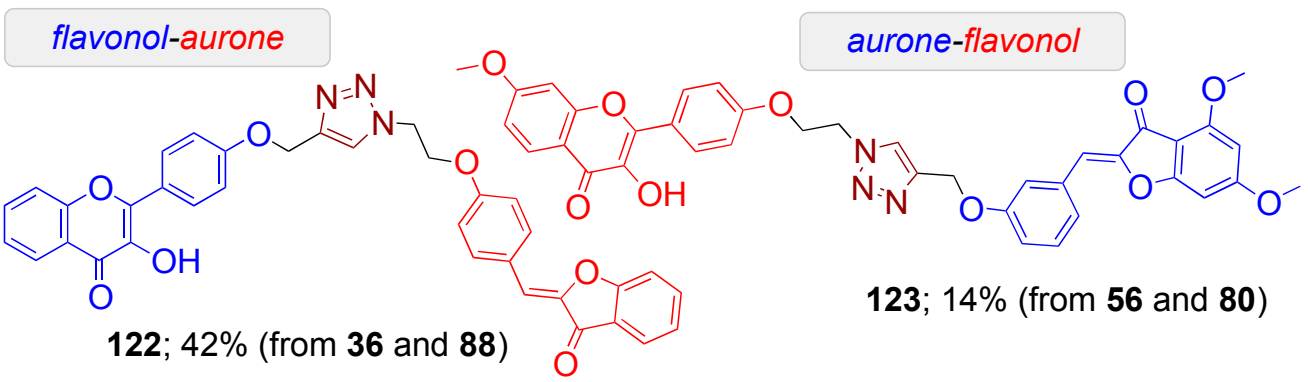

Scheme 20. Synthesis of some triazole-bridged dimers. 

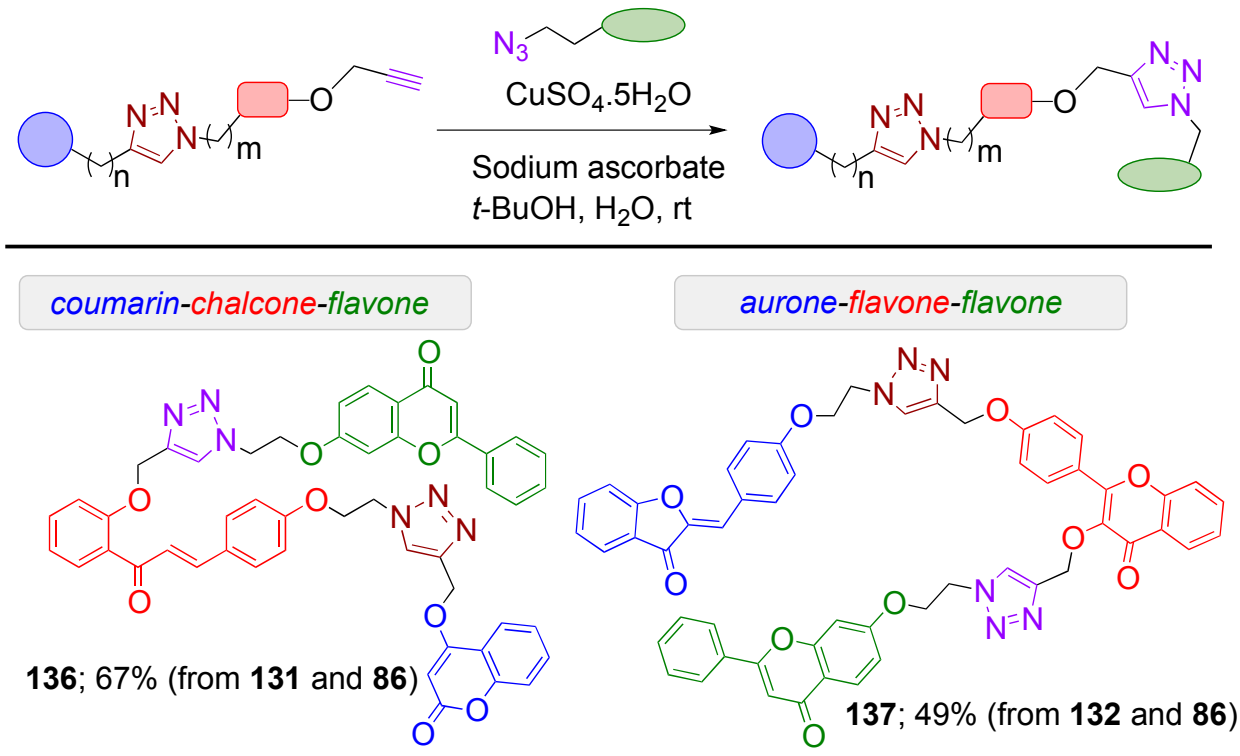

chalcone-chalcone-flavone

138; $5 \%$ (from

133 and 87)<smiles>CNCCOc1ccc(C(=O)/C=C/c2c(OC)cc(OC)cc2OC)cc1OC</smiles><smiles>C=C(Oc1ccccc1)c1ccc(OCCn2cc(COc3c(CC)oc4ccccc4c3=O)nn2)cc1</smiles><smiles>COc1ccccc1OCCn1cc(COc2cc(/C=C/C(=O)O)ccc2OC)nn1</smiles>

chavo-flavone-auro

139; 36\% (from 134 and 89)

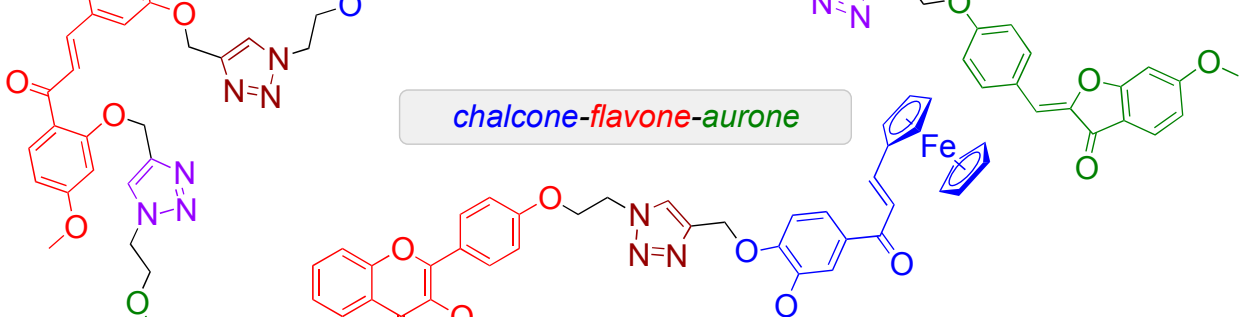<smiles>C=CCOc1ccc2c(=O)cc(-c3ccc(OCC)cc3)oc2c1</smiles>

$\mathrm{O}$<smiles>COCCn1cc(I)nn1</smiles>

140; $3 \%$ (from 135 and 89 )

Scheme 21. Synthesis of triazole-bridged trimers. 


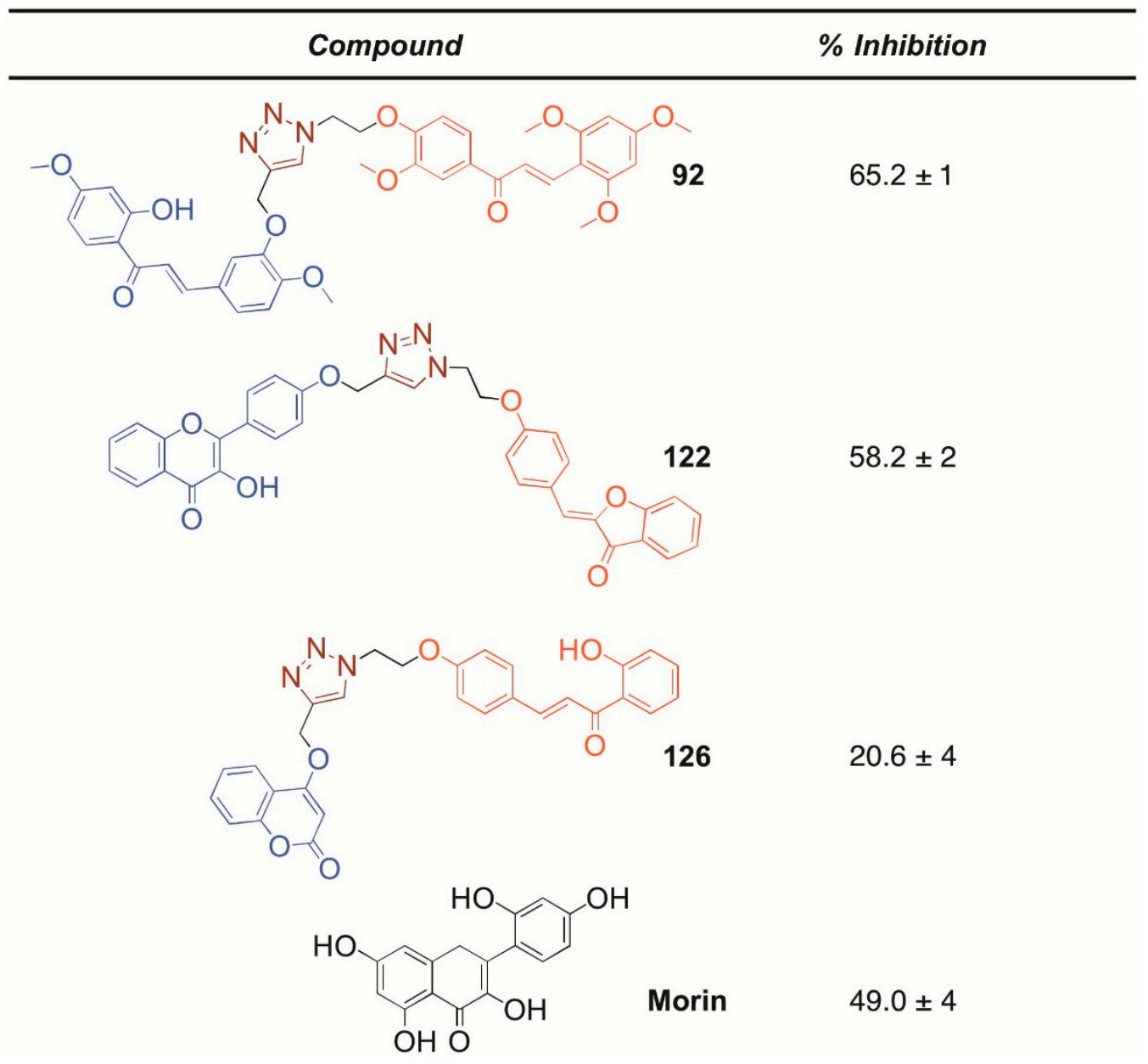

Figure 2. Percentage inhibition of $A \beta_{42}$ aggregation achieved by compounds $\mathbf{9 2}, \mathbf{1 2 2}$ and $\mathbf{1 2 6}(50 \mu \mathrm{M}$ concentration) relative to that of $\mathrm{A} \beta_{42}$ alone $(10 \mu \mathrm{M})$, where $100 \%$ represents complete aggregation inhibition and $0 \%$ shows no inhibition. The data represents the averages and standard error from the results of three independent biological repeats. Inhibitory effect of morin determined under identical assay conditions.

\section{Materials and Methods}

\subsection{Chemical Synthesis}

\subsubsection{General Information}

All non-aqueous reactions were performed under a constant stream of dry nitrogen using oven-dried glassware. Standard practices were employed when handling moisture and air-sensitive materials. All reagents and solvents were purchased from commercial sources and used without further purification unless otherwise stated. Room temperature refers to ambient temperature. Temperatures of $0{ }^{\circ} \mathrm{C}$ were maintained using an ice-water bath. Petroleum ether was distilled before use. Ethyl acetate and methanol were distilled from calcium hydride. Melting points were measured using a Büchi B545 melting point apparatus and are uncorrected. Thin layer chromatography (TLC) was performed on pre-coated silica gel GF254 plates (Merck, Kenilworth, NJ, USA). Infrared (IR) spectra were recorded on a Spectrum One (FT-IR) spectrophotometer (Perkin-Elmer, Waltham, MA, USA) with internal referencing. Absorption maxima $\left(\nu_{\max }\right)$ are reported in wavenumbers $\left(\mathrm{cm}^{-1}\right)$. Flash column chromatography was performed on silica gel (230-400 mesh). ${ }^{1} \mathrm{H}-\mathrm{NMR}$ and ${ }^{13} \mathrm{C}-\mathrm{NMR}$ were recorded on an Avance $500 \mathrm{MHz}$ instrument (Bruker, Billerica, MA, USA) in $\mathrm{CDCl}_{3}$ or $\left(\mathrm{CD}_{3}\right)_{2} \mathrm{CO}$. Chemical shifts ( $\delta$ ) are quoted in ppm, to the nearest $0.01 \mathrm{ppm}\left({ }^{1} \mathrm{H}-\mathrm{NMR}\right)$ or $0.1 \mathrm{ppm}\left({ }^{13} \mathrm{C}-\mathrm{NMR}\right)$ and are referenced 
to the residual non-deuterated solvent peak. ${ }^{1} \mathrm{H}-\mathrm{NMR}$ and ${ }^{13} \mathrm{C}-\mathrm{NMR}$ data for all compounds can be found in Supplementary Materials. LCMS analysis was performed on an ACQUITY H-Class UPLC (Waters, Milford, MA, USA) with an ESCi Multi-Mode Ionisation Waters SQ Detector 2 spectrometer using MassLynx 4.1 software. LC system: solvent A: $2 \mathrm{mM} \mathrm{NH}_{4} \mathrm{OAc}$ in $\mathrm{H}_{2} \mathrm{O} / \mathrm{MeCN}$ (95:5); solvent B: MeCN; solvent C: $2 \%$ aqueous formic acid; gradient: $5 \%-95 \%$ B with constant $5 \%$ Cover 1 min at flow rate of $0.6 \mathrm{~mL} / \mathrm{min}$. High resolution mass spectrometry (HRMS) measurements were recorded on a Q-TOF mass spectrometer (Micromass, Cary, NC, USA) or a Waters LCT Premier Time of Flight mass spectrometer. Mass values are quoted within the error limits of $\pm 5 \mathrm{ppm}$ mass units. ESI+ refers to the mass ionisation technique.

\subsubsection{General Synthetic Procedures}

General Procedure A: Synthesis of Biflavonoid Triazole Hybrids (GP-A). To a stirred solution of alkyne flavonoid (1.0 equiv.) and azide flavonoid (1.0 equiv.) in $t-\mathrm{BuOH} / \mathrm{H}_{2} \mathrm{O}(1: 1,40 \mathrm{~mL})$ were added $\mathrm{CuSO}_{4} \cdot 5 \mathrm{H}_{2} \mathrm{O}$ (1.1 equiv.) and sodium ascorbate (2.5 equiv.). The reaction mixture was stirred at room temperature for $24 \mathrm{~h}$ or until TLC analysis indicated complete consumption of starting material. The resulting mixture was poured into $\mathrm{H}_{2} \mathrm{O}(100 \mathrm{~mL})$ and the aqueous solution was extracted with $\mathrm{CHCl}_{3}(3 \times 100 \mathrm{~mL})$. The combined organic layer was washed with $\mathrm{H}_{2} \mathrm{O}(2 \times 100 \mathrm{~mL})$, brine $(2 \times 100 \mathrm{~mL})$, dried over anhydrous $\mathrm{MgSO}_{4}$, filtered and the solvent removed under reduced pressure. The crude residue was purified by flash column chromatography over silica and recrystallized from $\mathrm{MeOH}$ to afford the corresponding biflavonoid triazole hybrids.

General Procedure B: Synthesis of Alkyne Biflavonoid Triazole Hybrids (GP-B). To a stirred solution of the corresponding biflavonoid triazole hybrid (1.0 equiv.) in dry acetone $(50 \mathrm{~mL})$ were added propargyl bromide (3.0 equiv.) and anhydrous $\mathrm{K}_{2} \mathrm{CO}_{3}$ (3.0 equiv.). The reaction mixture was heated at reflux with stirring for $24 \mathrm{~h}$ under a nitrogen atmosphere or until TLC analysis indicated complete consumption of starting material. The resulting mixture was allowed to cool to room temperature and the solvent removed in vacuo. The crude residue was re-suspended in $\mathrm{CHCl}_{3}(50 \mathrm{~mL})$ and the organic layer was washed with $\mathrm{H}_{2} \mathrm{O}(2 \times 100 \mathrm{~mL})$, brine $(2 \times 100 \mathrm{~mL})$, dried over anhydrous $\mathrm{MgSO}_{4}$, filtered and evaporated to dryness. The crude residue was purified by flash column chromatography over silica to afford the corresponding propynyloxy biflavonoid triazole hybrid.

General Procedure C: Synthesis of Propynyloxy flavonoids or benzaldehydes (GP-C). To a stirred solution of the corresponding flavonoid or benzaldehyde (1.0 equiv.) in dry acetone (50 mL) were added anhydrous $\mathrm{K}_{2} \mathrm{CO}_{3}$ (3.0 equiv.) and propargyl bromide (3.0 equiv.). The reaction mixture was heated at reflux with stirring for $24 \mathrm{~h}$ under a nitrogen atmosphere or until TLC analysis indicated complete consumption of starting material. The resulting mixture was allowed to cool to room temperature and the solvent removed in vacuo. The crude residue was re-suspended in $\mathrm{CHCl}_{3}(100 \mathrm{~mL})$ and the organic layer was washed with $\mathrm{H}_{2} \mathrm{O}(2 \times 100 \mathrm{~mL})$, brine $(2 \times 100 \mathrm{~mL})$, dried over anhydrous $\mathrm{MgSO}_{4}$, filtered and evaporated to dryness. The crude residue was purified by flash column chromatography over silica to afford the corresponding propynyloxy flavonoids or benzaldehydes.

General Procedure D: Synthesis of Chalcones (GP-D). To a stirred solution of $\mathrm{KOH}$ (12.0 equiv.) in absolute EtOH $(100 \mathrm{~mL})$ cooled to $0{ }^{\circ} \mathrm{C}$ in an ice-bath were added dropwise a solution of the corresponding acetophenone (1.0 equiv.) and aldehyde (1.0 equiv.) in $\mathrm{EtOH}(20 \mathrm{~mL})$. The reaction mixture was stirred at $0{ }^{\circ} \mathrm{C}$ for $1 \mathrm{~h}$ and then at room temperature for $72 \mathrm{~h}$ under a nitrogen atmosphere or until TLC analysis indicated complete consumption of starting material. The resulting mixture was then poured into ice-water $(100 \mathrm{~mL})$ and acidified to $\mathrm{pH} 3-4$ with $3 \mathrm{M} \mathrm{HCl}$. The aqueous solution was extracted with $\mathrm{CHCl}_{3}(3 \times 100 \mathrm{~mL})$ and the combined organic layer was washed with satd $\mathrm{NaHCO}_{3}$ $(2 \times 100 \mathrm{~mL})$, brine $(2 \times 100 \mathrm{~mL})$, dried over anhydrous $\mathrm{MgSO}_{4}$, filtered and the solvent removed under reduced pressure. The crude residue was purified by flash column chromatography over silica and/or recrystallized from $\mathrm{MeOH}$ or absolute $\mathrm{EtOH}$ to afford the corresponding chalcones. 
General Procedure E: Synthesis of Indole or Pyrrole 2-hydroxychalcones (GP-E). To a stirred solution of indole or pyrrole aldehyde (1.0 equiv.) and the corresponding 2-hydroxyacetophenone (1.0 equiv.) in absolute $\mathrm{EtOH}(100 \mathrm{~mL})$ was added piperidine (1.0 equiv.). The reaction mixture was heated at reflux for $24 \mathrm{~h}$ under a nitrogen atmosphere or until TLC analysis indicated complete consumption of starting material. The reaction mixture was allowed to cool to room temperature, poured into ice-water (100 mL) and then acidified to $\mathrm{pH} 3-4$ with $3 \mathrm{M} \mathrm{HCl}$. The resulting suspension was filtered and the precipitate washed with ice-water $(2 \times 100 \mathrm{~mL})$, suction-dried and recrystallized from $\mathrm{MeOH}$ to afford the corresponding indole or pyrrole chalcones.

General Procedure F: Synthesis of Flavonols (GP-F). To a stirred solution of the corresponding chalcone $(0.30 \mathrm{mmol})$ in $\mathrm{MeOH}(20 \mathrm{~mL})$ were added $16 \% \mathrm{NaOH}(\mathrm{aq})(0.60 \mathrm{~mL})$ and $15 \% \mathrm{H}_{2} \mathrm{O}_{2}(0.30 \mathrm{~mL})$. The reaction mixture was stirred at room temperature for $24 \mathrm{~h}$ under a nitrogen atmosphere or until TLC analysis indicated complete consumption of starting material. The resulting mixture was then poured into ice-water $(50 \mathrm{~mL})$ and acidified to $\mathrm{pH} 3-4$ with $3 \mathrm{M} \mathrm{HCl}$. The aqueous solution was extracted with $\mathrm{CHCl}_{3}(3 \times 50 \mathrm{~mL})$ and the combined organic layer was washed with satd $\mathrm{NaHCO}_{3}$ $(2 \times 50 \mathrm{~mL})$, brine $(2 \times 50 \mathrm{~mL})$, dried over anhydrous $\mathrm{MgSO}_{4}$, filtered and the solvent removed under reduced pressure. The crude residue was purified by flash column chromatography over silica to afford the corresponding flavonols.

General Procedure G: Synthesis of Phenylethanones (GP-G). To a stirred solution of substituted phenol (1.2 equiv.) in $\mathrm{BF}_{3} \cdot \mathrm{OEt}_{2}(50 \mathrm{~mL})$ was added the corresponding phenylacetic acid (1.0 equiv.) and the reaction mixture was heated at $80{ }^{\circ} \mathrm{C}$ for $8 \mathrm{~h}$ under a nitrogen atmosphere. The resulting dark solution was allowed to cool to room temperature and slowly poured into ice-water $(100 \mathrm{~mL})$. The organic layer was separated and the aqueous layer was extracted with EtOAc $(3 \times 100 \mathrm{~mL})$. The combined organic layer was washed with satd $\mathrm{NaHCO}_{3}(2 \times 100 \mathrm{~mL})$, brine $(2 \times 100 \mathrm{~mL})$, dried over anhydrous $\mathrm{MgSO}_{4}$, filtered and evaporated to dryness. The crude residue was purified by flash column chromatography over silica to afford the corresponding phenylethanones.

General Procedure H: Synthesis of Isoflavones (GP-H). To a stirred solution of the corresponding phenylethanone (1.0 equiv.) in dry $\mathrm{DMF}\left(15 \mathrm{~mL}\right.$ ) was carefully added $\mathrm{BF}_{3} \cdot \mathrm{OEt}_{2}$ (4.0 equiv.) over $10 \mathrm{~min}$ under a nitrogen atmosphere. To this mixture, methanesulfonyl chloride (3.0 equiv.) was added at $55^{\circ} \mathrm{C}$, stirred for $1 \mathrm{~h}$ and then heated at $80{ }^{\circ} \mathrm{C}$ for $24 \mathrm{~h}$. The resulting dark solution was allowed to cool to room temperature and then poured with rapid stirring into ice-water $(100 \mathrm{~mL})$. The resulting precipitate was filtered, washed with $\mathrm{H}_{2} \mathrm{O}(2 \times 100 \mathrm{~mL})$, suction-dried and re-dissolved in EtOAc (100 mL). The organic solution was washed with $\mathrm{H}_{2} \mathrm{O}(2 \times 100 \mathrm{~mL})$, brine $(2 \times 100 \mathrm{~mL})$, dried over anhydrous $\mathrm{MgSO}_{4}$, filtered and evaporated to dryness. The crude residue was purified by flash column chromatography over silica to afford the corresponding isoflavones.

General Procedure I: Synthesis of Bromoalkylated flavonoids or benzaldehdyes (GP-I). To a stirred solution of the corresponding flavonoid or benzaldehyde (1.0 equiv.) in dry acetone $(50 \mathrm{~mL})$ or dry DMF $(50 \mathrm{~mL})$ were added anhydrous $\mathrm{K}_{2} \mathrm{CO}_{3}$ (3.0 equiv.) and 1,2-dibromoethane (3.0 equiv.). The reaction mixture was heated at reflux with stirring for $24 \mathrm{~h}$ under a nitrogen atmosphere or until TLC analysis indicated complete consumption of starting material. The resulting mixture was allowed to cool to room temperature and the solvent removed in vacuo. The crude residue was re-suspended in $\mathrm{CHCl}_{3}(100 \mathrm{~mL})$ and the organic layer was washed with $\mathrm{H}_{2} \mathrm{O}(2 \times 100 \mathrm{~mL})$, brine $(2 \times 100 \mathrm{~mL})$, dried over anhydrous $\mathrm{MgSO}_{4}$, filtered and evaporated to dryness. The crude residue was purified by flash column chromatography over silica to afford the corresponding bromoalkylated flavonoids or benzaldehydes.

General Procedure J: Synthesis of Azido flavonoids or benzaldehydes (GP-J). To a stirred solution of the corresponding bromoalkylated flavonoids or benzaldehydes (1.0 equiv.) in dry DMF (30 mL) was added $\mathrm{NaN}_{3}$ (3.0 equiv.). The reaction mixture was heated at $100{ }^{\circ} \mathrm{C}$ with stirring for $3 \mathrm{~h}$ under a nitrogen atmosphere. The resulting mixture was allowed to cool to room temperature and poured 
into $\mathrm{H}_{2} \mathrm{O}(100 \mathrm{~mL})$. The aqueous solution was extracted with $\mathrm{CHCl}_{3}(3 \times 100 \mathrm{~mL})$ and the combined organic layer was washed with $\mathrm{H}_{2} \mathrm{O}(2 \times 100 \mathrm{~mL})$, brine $(2 \times 100 \mathrm{~mL})$, dried over anhydrous $\mathrm{MgSO}_{4}$, filtered and evaporated to dryness to afford the corresponding azido flavonoids or benzaldehydes and were used without further purification.

General Procedure K: Synthesis of Aurones (GP-K). To a stirred solution of the corresponding chalcone (1.0 equiv.) in pyridine $(10 \mathrm{~mL})$ was added $\mathrm{Hg}(\mathrm{OAc})_{2}(1.0$ equiv.). The reaction mixture was heated at $110{ }^{\circ} \mathrm{C}$ with stirring for $1 \mathrm{~h}$ under a nitrogen atmosphere. The resulting mixture was then poured into ice-water $(50 \mathrm{~mL})$ and acidified to $\mathrm{pH} 3-4$ with $3 \mathrm{M} \mathrm{HCl}$. The aqueous solution was extracted with $\mathrm{CHCl}_{3}(3 \times 50 \mathrm{~mL})$ and the combined organic layer was washed with $\mathrm{H}_{2} \mathrm{O}(2 \times 50 \mathrm{~mL})$, brine $(2 \times 50 \mathrm{~mL})$, dried over anhydrous $\mathrm{MgSO}_{4}$, filtered and the solvent removed under reduced pressure. The crude residue was purified by flash column chromatography over silica to afford the corresponding aurones.

\subsection{Synthetic Procedures}

\subsubsection{Building Block Synthesis}

4-Methoxy-3-(prop-2-yn-1-yloxy)benzaldehyde (2). A mixture of isovanillin (1, $10.0 \mathrm{~g}, 65.7 \mathrm{mmol})$, propargyl bromide $(8.78 \mathrm{~mL}, 98.6 \mathrm{mmol})$ and anhydrous $\mathrm{K}_{2} \mathrm{CO}_{3}(18.2 \mathrm{~g}, 131 \mathrm{mmol})$ in dry acetone $(100 \mathrm{~mL})$ was reacted according to GP-C. The crude residue was purified by flash column chromatography $\left(\mathrm{SiO}_{2}, \mathrm{CH}_{2} \mathrm{Cl}_{2}\right)$ to afford benzaldehyde $2(9.23 \mathrm{~g}, 74 \%)$ as a white fluffy solid. m.p. $78-80^{\circ} \mathrm{C}$. TLC $R_{\mathrm{f}}=0.21\left(\mathrm{CH}_{2} \mathrm{Cl}_{2}\right)$. IR $v_{\max }$ (neat) $/ \mathrm{cm}^{-1}: 3229 \mathrm{~m}(\mathrm{C} \equiv \mathrm{C}-\mathrm{H} \mathrm{str}), 3096 \mathrm{w}(\mathrm{C}-\mathrm{H}$ str), 2839w (C-H str), 2124w (C $\equiv C$ str), 1671s ( $C=O$ str), 1599s ( $C=C$ str), 1586s ( $C=C$ str), 1508s (C=C str), 1436m, 1407m, 1387m, 1261s, 1229s, 1161s, 1129s, 1012s. ${ }^{1} \mathrm{H}-\mathrm{NMR}\left(500 \mathrm{MHz}, \mathrm{CDCl}_{3}\right): \delta 2.55(1 \mathrm{H}, \mathrm{t}$, $\left.J=2.4 \mathrm{~Hz},-\mathrm{OCH}_{2} \mathrm{C} \equiv \mathrm{CH}\right), 3.97\left(3 \mathrm{H}, \mathrm{s},-\mathrm{OCH}_{3}\right), 4.83\left(2 \mathrm{H}, \mathrm{d}, J=2.4 \mathrm{~Hz},-\mathrm{OCH}_{2} \mathrm{C} \equiv \mathrm{CH}\right), 7.01(1 \mathrm{H}, \mathrm{d}$, $J=8.0 \mathrm{~Hz}, \mathrm{ArH}), 7.53(1 \mathrm{H}, \mathrm{dd}, J=8.0,1.6 \mathrm{~Hz}, \mathrm{ArH}), 7.55(1 \mathrm{H}, \mathrm{d}, J=1.6 \mathrm{~Hz}, \mathrm{ArH}), 9.87(1 \mathrm{H}, \mathrm{s}, \mathrm{CHO})$. ${ }^{13} \mathrm{C}-\mathrm{NMR}\left(500 \mathrm{MHz}, \mathrm{CDCl}_{3}\right): \delta 56.2,56.6,76.4,77.7,110.9,112.0,127.3,129.9,147.3,154.9,190.7$. LCMS $(\mathrm{ES}+) m / z=191.2\left([\mathrm{M}+\mathrm{H}]^{+}, t_{\mathrm{R}}=3.09 \mathrm{~min}\right)$. These characterisation data are in accordance with that previously reported in the literature [37].

(E)-1-(2-Hydroxy-4-methoxyphenyl)-3-(4-methoxy-3-(prop-2-yn-1-yloxy)phenyl)prop-2-en-1-one (4). A mixture of benzaldehyde $2(2.05 \mathrm{~g}, 10.8 \mathrm{mmol})$, acetophenone $3(1.80 \mathrm{~g}, 10.8 \mathrm{mmol})$ and $\mathrm{KOH}(3.02 \mathrm{~g}, 53.8 \mathrm{mmol})$ in absolute $\mathrm{EtOH}(100 \mathrm{~mL})$ was reacted according to GP-D. The crude residue was purified by flash column chromatography $\left(\mathrm{SiO}_{2}, \mathrm{PE} / \mathrm{EtOAc} ; 5: 1\right)$ and recrystallized from $\mathrm{MeOH}$ to afford chalcone 4 $(1.42 \mathrm{~g}, 39 \%)$ as a bright yellow-orange fluffy solid. m.p. $144-146{ }^{\circ} \mathrm{C}$. TLC $R_{\mathrm{f}}=0.37$ (PE/EtOAc; $2: 1$ ). IR $v_{\max }$ (neat) $/ \mathrm{cm}^{-1}: 3506 \mathrm{w}$ (br) (O-H str), $3229 \mathrm{~m}$ (C $\equiv \mathrm{C}-\mathrm{H}$ str), 3081w (C-H str), 2973w (C-H str), $2111 \mathrm{w}$ ( $\mathrm{C} \equiv \mathrm{C}$ str), $1633 \mathrm{~m}$ (C=O str), $1572 \mathrm{~m}$ (C=C str), 1556s (C=C str), 1507s (C=C str), $1466 \mathrm{~m}, 1440 \mathrm{~s}$, 1361s, 1339s, 1260s, 1206s, 1170m, 1126s, 1018s. ${ }^{1} \mathrm{H}-\mathrm{NMR}\left(500 \mathrm{MHz}, \mathrm{CDCl}_{3}\right): \delta 2.58(1 \mathrm{H}, \mathrm{t}, J=2.0 \mathrm{~Hz}$, $\left.-\mathrm{OCH}_{2} \mathrm{C} \equiv \mathrm{CH}\right), 3.87\left(3 \mathrm{H}, \mathrm{s},-\mathrm{OCH}_{3}\right), 3.94\left(3 \mathrm{H}, \mathrm{s},-\mathrm{OCH}_{3}\right), 4.85\left(2 \mathrm{H}, \mathrm{d}, J=2.4 \mathrm{~Hz},-\mathrm{OCH}_{2} \mathrm{C} \equiv \mathrm{CH}\right)$, 6.49-6.52 (2H, m, ArH), $6.94(1 \mathrm{H}, \mathrm{d}, J=8.4 \mathrm{~Hz}, \mathrm{ArH}), 7.32(1 \mathrm{H}, \mathrm{dd}, J=8.4,2.0 \mathrm{~Hz}, \mathrm{ArH}), 7.35(1 \mathrm{H}, \mathrm{d}$, $J=1.2 \mathrm{~Hz}, \mathrm{ArH}), 7.45(1 \mathrm{H}, \mathrm{d}, J=15.2 \mathrm{~Hz},-\mathrm{CH}=\mathrm{CHCO}-), 7.83(1 \mathrm{H}, \mathrm{d}, J=8.4 \mathrm{~Hz}, \mathrm{ArH}), 7.85(1 \mathrm{H}, \mathrm{d}$, $J=15.6 \mathrm{~Hz},-\mathrm{CH}=\mathrm{CHCO}-), 13.53(1 \mathrm{H}, \mathrm{s}, \mathrm{OH}) .{ }^{13} \mathrm{C}-\mathrm{NMR}\left(500 \mathrm{MHz}, \mathrm{CDCl}_{3}\right): \delta 55.6,56.0,56.9,76.3,78.1$, 101.0, 107.7, 111.7, 113.8, 114.1, 118.3, 124.3, 127.7, 131.1, 144.3, 146.9, 152.2, 166.1, 166.6, 191.7. LCMS $(\mathrm{ES}+) \mathrm{m} / z=339.2\left([\mathrm{M}+\mathrm{H}]^{+}, t_{\mathrm{R}}=1.70 \mathrm{~min}\right)$. HRMS (ESI+) $\mathrm{m} / z=339.1224[\mathrm{M}+\mathrm{H}]^{+}$found, $\mathrm{C}_{20} \mathrm{H}_{19} \mathrm{O}_{5}{ }^{+}$ required 339.1227 .

(E)-1-(4-Hydroxy-3-methoxyphenyl)-3-(4-methoxyphenyl)prop-2-en-1-one (13). A mixture of aldehyde 5 $(7.40 \mathrm{~mL}, 60.8 \mathrm{mmol})$, acetophenone $11(10.0 \mathrm{~g}, 60.3 \mathrm{mmol})$ and $\mathrm{KOH}(16.9 \mathrm{~g}, 302 \mathrm{mmol})$ in absolute $\mathrm{EtOH}(100 \mathrm{~mL})$ was reacted according to GP-D. The crude residue was purified by flash column chromatography $\left(\mathrm{SiO}_{2}, \mathrm{PE} / \mathrm{EtOAc} ; 5: 1\right)$ to afford chalcone $\mathbf{1 3}(10.7 \mathrm{~g}, 63 \%)$ as a bright yellow-orange powdery solid. m.p. $148-150{ }^{\circ} \mathrm{C}$. TLC $R_{\mathrm{f}}=0.40$ (PE/EtOAc; $\left.1: 1\right)$. IR $v_{\max }$ (neat) $/ \mathrm{cm}^{-1}: 3180 \mathrm{~m}(\mathrm{br})$ (O-H str), 2991w (C-H str), 2836w (C-H str), 1643m (C=O str), 1602m (C=C str), 1560s (C=C str), 1508s 
(C=C str), 1461w, 1424s, 1338m, 1296s, 1278s, 1248s, 1166s, 1048m, 1026s. ${ }^{1} \mathrm{H}-\mathrm{NMR}\left(500 \mathrm{MHz}, \mathrm{CDCl}_{3}\right)$ : $\delta 3.86\left(3 \mathrm{H}, \mathrm{s},-\mathrm{OCH}_{3}\right), 3.98\left(3 \mathrm{H}, \mathrm{s},-\mathrm{OCH}_{3}\right), 6.23(1 \mathrm{H}, \mathrm{s}, \mathrm{OH}), 6.94(2 \mathrm{H}, \mathrm{d}, J=9.0 \mathrm{~Hz}, \mathrm{ArH}), 7.00(1 \mathrm{H}$, $\mathrm{d}, J=8.0 \mathrm{~Hz}, \mathrm{ArH}), 7.45(1 \mathrm{H}, \mathrm{d}, J=15.5 \mathrm{~Hz},-\mathrm{CH}=\mathrm{CHCO}-), 7.61(2 \mathrm{H}, \mathrm{d}, J=8.5 \mathrm{~Hz}, \mathrm{ArH}), 7.64-7.66$ $(2 \mathrm{H}, \mathrm{m}, \mathrm{ArH}), 7.79(1 \mathrm{H}, \mathrm{d}, J=16.0 \mathrm{~Hz},-\mathrm{CH}=\mathrm{CHCO}-) .{ }^{13} \mathrm{C}-\mathrm{NMR}\left(500 \mathrm{MHz}, \mathrm{CDCl}_{3}\right): \delta 55.4,56.1,110.5$, $113.7,114.3,119.3,123.5,127.8,130.1,131.2,143.8,146.8,150.2,161.5,188.6$. LCMS (ES+) $m / z=285.2$ $\left([\mathrm{M}+\mathrm{H}]^{+}, t_{\mathrm{R}}=1.75 \mathrm{~min}\right)$. These characterisation data are in accordance with that previously reported in the literature [38].

(E)-1-(5-Bromo-2-hydroxyphenyl)-3-(1-methyl-1H-indol-3-yl)prop-2-en-1-one (14). A mixture of indole aldehyde $6(1.03 \mathrm{~g}, 6.47 \mathrm{mmol})$, acetophenone $12(1.38 \mathrm{~g}, 6.42 \mathrm{mmol})$ and piperidine $(0.62 \mathrm{~mL}$, $6.28 \mathrm{mmol})$ in absolute $\mathrm{EtOH}(100 \mathrm{~mL})$ was reacted according to GP-E. The crude residue was purified by recrystallization from $\mathrm{MeOH}$ to afford chalcone $14(2.15 \mathrm{~g}, 94 \%)$ as a bright orange fluffy solid. m.p. 250-252 ${ }^{\circ} \mathrm{C}$. TLC $R_{\mathrm{f}}=0.45$ (PE/EtOAc 2:1). IR $v_{\max }$ (neat) $/ \mathrm{cm}^{-1}$ : 3101w (C-H str), 2885w (C-H str), 1626s (C=O str), 1541m (C=C str), 1513s (C=C str), 1462m, 1387m, 1375m, 1344m, 1295m, 1252s, 1177s, 1126s, 1074s, 1028s, 1014m. ${ }^{1} \mathrm{H}-\mathrm{NMR}\left(500 \mathrm{MHz}, \mathrm{CDCl}_{3}\right): \delta 3.88\left(3 \mathrm{H}, \mathrm{s},-\mathrm{NCH}_{3}\right), 6.93$ $(1 \mathrm{H}, \mathrm{d}, J=8.8 \mathrm{~Hz}, \mathrm{ArH}), 7.38-7.40(3 \mathrm{H}, \mathrm{m}, \mathrm{ArH}), 7.49-7.56(2 \mathrm{H}, \mathrm{m}, \mathrm{ArH}), 7.55(1 \mathrm{H}, \mathrm{d}, J=15.6 \mathrm{~Hz}$, -CH=CHCO-), 8.00-8.03 (2H, m, ArH), 8.23 (1H, d, J = 15.2 Hz, -CH=CHCO-), 13.25 (1H, br s, OH). ${ }^{13} \mathrm{C}-\mathrm{NMR}\left(500 \mathrm{MHz}, \mathrm{CDCl}_{3}\right): \delta 33.5,110.2,110.3,113.1,113.6,120.5,120.8,121.7,122.1,123.6,126.1$, $131.5,135.6,138.1,138.4,140.4,162.4,192.5$. LCMS (ES+) $m / z=358.0\left([\mathrm{M}+\mathrm{H}]^{+}, t_{\mathrm{R}}=4.96 \mathrm{~min}\right)$. HRMS (ESI+) $m / z=356.0279[\mathrm{M}+\mathrm{H}]^{+}$found, $\mathrm{C}_{18} \mathrm{H}_{15} \mathrm{O}_{2} \mathrm{NBr}^{+}$required 356.0281.

(E)-1-(2-Hydroxyphenyl)-3-(1-methyl-1H-pyrrol-2-yl)prop-2-en-1-one (15). A mixture of pyrrole aldehyde $7(4.00 \mathrm{~mL}, 37.2 \mathrm{mmol})$, acetophenone $10(4.50 \mathrm{~mL}, 37.4 \mathrm{mmol})$ and piperidine $(3.80 \mathrm{~mL}, 38.5 \mathrm{mmol})$ in absolute EtOH (100 mL) was reacted according to GP-E. The crude residue was purified by recrystallization from $\mathrm{MeOH}$ to afford chalcone $15(2.28 \mathrm{~g}, 27 \%)$ as a bright yellow-orange powdery solid. m.p. $98-100{ }^{\circ} \mathrm{C}$. TLC $R_{\mathrm{f}}=0.45$ (PE/EtOAc; 3:1). IR $v_{\max }$ (neat) $/ \mathrm{cm}^{-1}: 3112 \mathrm{w}$ (C-H str), 2937w (C-H str), 1627s (C=O str), 1579w (C=C str), 1549s (C=C str), 1479s, 1440m, 1412m, 1384m, 1356w, 1338m, 1290m, 1260s, 1249s, 1203s, 1182w, 1155s, 1092w, 1061s, 1025s. ${ }^{1} \mathrm{H}-\mathrm{NMR}\left(500 \mathrm{MHz}, \mathrm{CDCl}_{3}\right)$ : $\delta 3.81\left(3 \mathrm{H}, \mathrm{s},-\mathrm{NCH}_{3}\right), 6.26-6.27(1 \mathrm{H}, \mathrm{m}, \mathrm{ArH}), 6.87(1 \mathrm{H}, \mathrm{t}, J=2.0 \mathrm{~Hz}, \mathrm{ArH}), 6.91-6.96(2 \mathrm{H}, \mathrm{m}, \mathrm{ArH})$, $7.02(1 \mathrm{H}, \mathrm{dd}, J=8.4,0.8 \mathrm{~Hz}, \mathrm{ArH}), 7.40(1 \mathrm{H}, \mathrm{d}, J=14.8 \mathrm{~Hz},-\mathrm{CH}=\mathrm{CHCO}-), 7.48(1 \mathrm{H}, \mathrm{t}, J=8.4 \mathrm{~Hz}, \mathrm{ArH})$, $7.89(1 \mathrm{H}, \mathrm{dd}, J=8.0,2.0 \mathrm{~Hz}, \mathrm{ArH}), 7.92(1 \mathrm{H}, \mathrm{d}, J=14.8 \mathrm{~Hz},-\mathrm{CH}=\mathrm{CHCO}-), 13.14(1 \mathrm{H}, \mathrm{s}, \mathrm{OH}) .{ }^{13} \mathrm{C}-\mathrm{NMR}$ (500 MHz, $\left.\mathrm{CDCl}_{3}\right): \delta 34.4,110.2,113.3,114.5,118.5,118.7,120.2,128.6,129.2,130.2,132.8,135.8,163.4$, 193.1. LCMS (ES+) $m / z=228.1\left([\mathrm{M}+\mathrm{H}]^{+}, t_{\mathrm{R}}=1.68 \mathrm{~min}\right)$. HRMS (ESI+) $m / z=228.1020[\mathrm{M}+\mathrm{H}]^{+}$ found, $\mathrm{C}_{14} \mathrm{H}_{14} \mathrm{O}_{2} \mathrm{~N}^{+}$required 228.1019 .

(E)-1-(4-Hydroxy-3-methoxyphenyl)-3-(1-methyl-1H-pyrrol-2-yl)prop-2-en-1-one (16). A mixture of pyrrole aldehyde 7 (3.30 mL, $30.7 \mathrm{mmol})$, acetophenone 11 (5.02 g, $30.2 \mathrm{mmol})$ and $\mathrm{KOH}(10.2 \mathrm{~g}, 182 \mathrm{mmol})$ in absolute EtOH (100 mL) was reacted according to GP-D. The crude residue was purified by flash column chromatography $\left(\mathrm{SiO}_{2}, \mathrm{PE} /\right.$ EtOAc; 5:1) and recrystallized from absolute EtOH to afford chalcone $16(4.28 \mathrm{~g}, 55 \%)$ as a dark red-brown powdery solid. m.p. $150-152{ }^{\circ} \mathrm{C}$. TLC $R_{\mathrm{f}}=0.31$ (PE/EtOAc; 1:1). IR $v_{\max }$ (neat) $/ \mathrm{cm}^{-1}: 3177 \mathrm{~m}$ (br) (O-H str), 2958w (C-H str), 2930w (C-H str), 1630m (C=O str), 1600m (C=C str), 1587m (C=C str), 1543s (C=C str), 1508s (C=C str), 1478m, 1380w, 1333m, 1270s, 1218m, 1196s, 1169s, 1052s, 1031s. ${ }^{1} \mathrm{H}-\mathrm{NMR}\left(500 \mathrm{MHz}, \mathrm{CDCl}_{3}\right): \delta 3.77\left(3 \mathrm{H}, \mathrm{s},-\mathrm{NCH}_{3}\right), 3.99$ $\left(3 \mathrm{H}, \mathrm{s},-\mathrm{OCH}_{3}\right), 6.18(1 \mathrm{H}, \mathrm{s}, \mathrm{OH}), 6.23(1 \mathrm{H}, \mathrm{t}, J=3.2 \mathrm{~Hz}, \mathrm{ArH}), 6.81(1 \mathrm{H}, \mathrm{t}, J=2.0 \mathrm{~Hz}, \mathrm{ArH}), 6.84$ $(1 \mathrm{H}, \mathrm{dd}, J=4.0,1.6 \mathrm{~Hz}, \mathrm{ArH}), 6.99(1 \mathrm{H}, \mathrm{d}, J=8.8 \mathrm{~Hz}, \mathrm{ArH}), 7.32(1 \mathrm{H}, \mathrm{d}, J=15.2 \mathrm{~Hz},-\mathrm{CH}=\mathrm{CHCO}-)$, 7.62-7.64 (2H, m, ArH), $7.81(1 \mathrm{H}, \mathrm{d}, J=14.8 \mathrm{~Hz},-\mathrm{CH}=\mathrm{CHCO}-) .{ }^{13} \mathrm{C}-\mathrm{NMR}\left(500 \mathrm{MHz}, \mathrm{CDCl}_{3}\right): \delta 34.3$, $56.1,109.6,110.4,112.0,113.7,116.4,123.1,127.5,130.3,131.5,131.5,146.8,150.0,188.1$. LCMS (ES+) $m / z=258.1\left([\mathrm{M}+\mathrm{H}]^{+}, t_{\mathrm{R}}=1.40 \mathrm{~min}\right)$. HRMS (ESI+) $m / z=280.0935[\mathrm{M}+\mathrm{Na}]^{+}$found, $\mathrm{C}_{15} \mathrm{H}_{15} \mathrm{O}_{3} \mathrm{NNa}^{+}$ required 280.0944 .

(E)-1-(5-Bromo-2-hydroxyphenyl)-3-(furan-2-yl)prop-2-en-1-one (17). A mixture of furan aldehyde 8 $(1.20 \mathrm{~mL}, 14.5 \mathrm{mmol})$, acetophenone $12(3.06 \mathrm{~g}, 14.2 \mathrm{mmol})$ and $\mathrm{KOH}(4.70 \mathrm{~g}, 83.8 \mathrm{mmol})$ in absolute 
EtOH (100 mL) was reacted according to GP-D. The crude residue was purified by recrystallization from $\mathrm{MeOH}$ to afford chalcone $17(1.97 \mathrm{~g}, 47 \%)$ as a bright yellow-orange fluffy solid. m.p. $80-82{ }^{\circ} \mathrm{C}$. TLC $R_{\mathrm{f}}=0.50$ (PE/EtOAc; 3:1). IR $v_{\max }$ (neat) $/ \mathrm{cm}^{-1}: 3128 \mathrm{w}$ (C-H str), 3046w (C-H str), 2868w (C-H str), 1638m (C=O str), 1567s (C=C str), 1548s (C=C str), 1467s, 1364m, 1335s, 1296m, 1258s, 1202s, 1177s, 1077w, 1013s. ${ }^{1} \mathrm{H}-\mathrm{NMR}\left(500 \mathrm{MHz}, \mathrm{CDCl}_{3}\right): \delta 6.56-6.57(1 \mathrm{H}, \mathrm{m}, \mathrm{ArH}), 6.82(1 \mathrm{H}, \mathrm{d}, J=3.6 \mathrm{~Hz}$, ArH), $6.93(1 \mathrm{H}, \mathrm{d}, J=8.8 \mathrm{~Hz}, \mathrm{ArH}), 7.45(1 \mathrm{H}, \mathrm{d}, J=15.2 \mathrm{~Hz},-\mathrm{CH}=\mathrm{CHCO}-), 7.56(1 \mathrm{H}, \mathrm{dd}, J=9.2$, $2.4 \mathrm{~Hz}, \mathrm{ArH}), 7.60(1 \mathrm{H}, \mathrm{d}, J=0.8 \mathrm{~Hz}, \mathrm{ArH}), 7.70(1 \mathrm{H}, \mathrm{d}, J=15.2 \mathrm{~Hz},-\mathrm{CH}=\mathrm{CHCO}-), 8.00(1 \mathrm{H}, \mathrm{d}$, $J=2.4 \mathrm{~Hz}, \mathrm{ArH}), 12.83(1 \mathrm{H}, \mathrm{s}, \mathrm{OH}) .{ }^{13} \mathrm{C}-\mathrm{NMR}\left(500 \mathrm{MHz}, \mathrm{CDCl}_{3}\right): \delta 110.4,113.0,116.8,117.8,120.5$, $121.2,131.8,131.9,138.8,145.8,151.3,162.4,192.3$. LCMS $(\mathrm{ES}+) \mathrm{m} / z=293.1\left([\mathrm{M}+\mathrm{H}]^{+}, t_{\mathrm{R}}=1.85 \mathrm{~min}\right)$. These characterisation data are in accordance with that previously reported in the literature [39].

(E)-3-(Ferrocenyl)-1-(2-hydroxyphenyl)prop-2-en-1-one (18). A mixture of ferrocene aldehyde 9 (4.77 g, $22.3 \mathrm{mmol})$, acetophenone $10(2.70 \mathrm{~mL}, 22.4 \mathrm{mmol})$ and $\mathrm{KOH}(7.63 \mathrm{~g}, 136 \mathrm{mmol})$ in absolute EtOH $(100 \mathrm{~mL})$ was reacted according to GP-D. The crude residue was purified by recrystallization from $\mathrm{MeOH}$ to afford chalcone $18(4.99 \mathrm{~g}, 67 \%)$ as a dark purple microcrystalline solid. m.p. $162-164{ }^{\circ} \mathrm{C}$. TLC $R_{\mathrm{f}}=0.43$ (PE/EtOAc; 7:1). IR $v_{\max }$ (neat) $/ \mathrm{cm}^{-1}$ : 3315w(br) (O-H str), 3088w (C-H str), 1742w (C=O str), 1627m, 1554s (C=C str), 1489s, 1440m, 1383w, 1347m, 1302s, 1271m, 1246m, 1206s, 1158m, 1104m, 1048w, 1023m, 1002w. ${ }^{1} \mathrm{H}-\mathrm{NMR}\left(500 \mathrm{MHz}, \mathrm{CDCl}_{3}\right): \delta 4.21\left(5 \mathrm{H}, \mathrm{s},-\mathrm{C}_{5} \mathrm{H}_{5}\right), 4.55(2 \mathrm{H}, \mathrm{t}, J=2.0 \mathrm{~Hz}$, $\left.-\mathrm{C}_{5} \mathrm{H}_{4}\right), 4.65\left(2 \mathrm{H}, \mathrm{t}, J=2.0 \mathrm{~Hz},-\mathrm{C}_{5} \mathrm{H}_{4}\right), 6.94(1 \mathrm{H}, \mathrm{t}, J=8.0 \mathrm{~Hz}, \mathrm{ArH}), 7.03(1 \mathrm{H}, \mathrm{dd}, J=8.5,1.0 \mathrm{~Hz}$, $\mathrm{ArH}), 7.26\left(1 \mathrm{H}, \mathrm{d}, J=15.0 \mathrm{~Hz},-\mathrm{CH}=\mathrm{CHCO}-\right.$, overlain by $\left.\mathrm{CDCl}_{3}\right), 7.49(1 \mathrm{H}, \mathrm{t}, J=8.5 \mathrm{~Hz}, \mathrm{ArH}), 7.87$ $(1 \mathrm{H}, \mathrm{dd}, J=8.0,1.0 \mathrm{~Hz}, \mathrm{ArH}), 7.91(1 \mathrm{H}, \mathrm{d}, J=15.0 \mathrm{~Hz},-\mathrm{CH}=\mathrm{CHCO}-), 13.07(1 \mathrm{H}, \mathrm{s}, \mathrm{OH}) .{ }^{13} \mathrm{C}-\mathrm{NMR}$ $\left(500 \mathrm{MHz}_{,} \mathrm{CDCl}_{3}\right): \delta 69.3,69.9,71.8,78.8,116.7,118.6,118.6,120.0,129.3,135.9,147.9,163.6,192.7$. LCMS $(\mathrm{ES}+) m / z=333.1\left([\mathrm{M}+\mathrm{H}]^{+}, t_{\mathrm{R}}=1.87 \mathrm{~min}\right)$. These characterisation data are in accordance with that previously reported in the literature [40].

(E)-3-(Ferrocenyl)-1-(4-hydroxy-3-methoxyphenyl)prop-2-en-1-one (19). A mixture of ferrocene aldehyde 9 (3.86 g, $18.0 \mathrm{mmol})$, acetophenone 11 (3.05 g, $18.4 \mathrm{mmol})$ and $\mathrm{KOH}(6.10 \mathrm{~g}, 109 \mathrm{mmol})$ in absolute EtOH (100 mL) was reacted according to GP-D. The crude residue was purified by flash column chromatography $\left(\mathrm{SiO}_{2}, \mathrm{PE} /\right.$ EtOAc; $\left.5: 1\right)$ to afford chalcone $19(5.61 \mathrm{~g}, 86 \%)$ as a dark purple microcrystalline solid. m.p. $78-80^{\circ} \mathrm{C}$. TLC $R_{\mathrm{f}}=0.29$ (PE/EtOAc; $\left.2: 1\right)$. IR $v_{\max }$ (neat) $/ \mathrm{cm}^{-1}: 3098 \mathrm{w}(\mathrm{C}-\mathrm{H}$ str), 2835w (C-H str), 1743w, 1642m, (C=O str) 1588s (C=C str), 1563s (C=C str), 1511s (C=C str), 1462m, 1424s, 1359m, 1347m, 1281s, 1264s, 1189s, 1162s, 1026s, 1001w. ${ }^{1} \mathrm{H}-\mathrm{NMR}\left(500 \mathrm{MHz}, \mathrm{CDCl}_{3}\right): \delta .3 .95$ $\left(3 \mathrm{H}, \mathrm{s},-\mathrm{OCH}_{3}\right), 4.17\left(5 \mathrm{H}, \mathrm{s},-\mathrm{C}_{5} \mathrm{H}_{5}\right), 4.47\left(2 \mathrm{H}, \mathrm{t}, \mathrm{J}=2.0 \mathrm{~Hz},-\mathrm{C}_{5} \mathrm{H}_{4}\right), 4.59\left(2 \mathrm{H}, \mathrm{t}, \mathrm{J}=2.0 \mathrm{~Hz},-\mathrm{C}_{5} \mathrm{H}_{4}\right), 6.54$ $(1 \mathrm{H}$, br s, OH), $7.00(1 \mathrm{H}, \mathrm{d}, J=8.0 \mathrm{~Hz}, \mathrm{ArH}), 7.16(1 \mathrm{H}, \mathrm{d}, J=15.0 \mathrm{~Hz},-\mathrm{CH}=\mathrm{CHCO}-), 7.60$ (1H, dd, $J=8.5,1.5 \mathrm{~Hz}, \mathrm{ArH}), 7.63(1 \mathrm{H}, \mathrm{d}, J=1.5 \mathrm{~Hz}, \mathrm{ArH}), 7.76(1 \mathrm{H}, \mathrm{d}, J=15.5 \mathrm{~Hz},-\mathrm{CH}=\mathrm{CHCO}-) .{ }^{13} \mathrm{C}-\mathrm{NMR}$ (500 MHz, $\left.\mathrm{CDCl}_{3}\right): \delta 56.0,68.8,69.7,71.2,79.3,110.5,113.8,118.5,123.2,131.1,145.8,146.8,150.1,187.9$. LCMS (ES+) $m / z=363.1\left([\mathrm{M}+\mathrm{H}]^{+}, t_{\mathrm{R}}=1.66 \mathrm{~min}\right)$. HRMS (ESI+) $m / z=363.0595[\mathrm{M}+\mathrm{H}]^{+}$found, $\mathrm{C}_{20} \mathrm{H}_{19} \mathrm{O}_{3} \mathrm{Fe}^{+}$required 363.0600 .

(E)-1-(3-Methoxy-4-(prop-2-yn-1-yloxy)phenyl)-3-(4-methoxyphenyl)prop-2-en-1-one (20). A mixture of chalcone 13 (1.02 g, $3.57 \mathrm{mmol})$, propargyl bromide $(0.63 \mathrm{~mL}, 7.07 \mathrm{mmol})$ and anhydrous $\mathrm{K}_{2} \mathrm{CO}_{3}$ $(1.49 \mathrm{~g}, 10.8 \mathrm{mmol})$ in dry acetone $(50 \mathrm{~mL})$ was reacted according to GP-C. The crude residue was purified by flash column chromatography $\left(\mathrm{SiO}_{2}, \mathrm{CH}_{2} \mathrm{Cl}_{2}\right)$ to afford chalcone $20(1.07 \mathrm{~g}$, 93\%) as a pale yellow powdery solid. m.p. $168-170{ }^{\circ} \mathrm{C}$. TLC $R_{\mathrm{f}}=0.41\left(\mathrm{CH}_{2} \mathrm{Cl}_{2}\right)$. IR $v_{\max }$ (neat) $/ \mathrm{cm}^{-1}: 3251 \mathrm{w}$ (C $\equiv$ C-H str), 2990w (C-H str), 2835w (C-H str), 2116w (C $\equiv \mathrm{C}$ str), 1651s (C=O str), 1598s (C=C str), $1577 \mathrm{~s}(\mathrm{C}=\mathrm{C}$ str), 1508s (C=C str), 1466m, 1421s, 1382w, 1323m, 1257s, 1229s, 1165s, 1145s, 1056w, 1015s. ${ }^{1} \mathrm{H}-\mathrm{NMR}\left(500 \mathrm{MHz}, \mathrm{CDCl}_{3}\right): \delta 2.57\left(1 \mathrm{H}, \mathrm{t}, J=2.4 \mathrm{~Hz},-\mathrm{OCH}_{2} \mathrm{C} \equiv \mathrm{CH}\right), 3.87\left(3 \mathrm{H}, \mathrm{s},-\mathrm{OCH}_{3}\right), 3.98(3 \mathrm{H}, \mathrm{s}$, $\left.-\mathrm{OCH}_{3}\right), 4.87\left(2 \mathrm{H}, \mathrm{d}, J=2.4 \mathrm{~Hz},-\mathrm{OCH}_{2} \mathrm{C} \equiv \mathrm{CH}\right), 6.95(2 \mathrm{H}, \mathrm{d}, J=8.8 \mathrm{~Hz}, \mathrm{ArH}), 7.11(2 \mathrm{H}, \mathrm{d}, J=8.4 \mathrm{~Hz}$, $\mathrm{ArH}), 7.44(1 \mathrm{H}, \mathrm{d}, J=15.6 \mathrm{~Hz},-\mathrm{CH}=\mathrm{CHCO}-), 7.62(2 \mathrm{H}, \mathrm{d}, J=8.8 \mathrm{~Hz}, \mathrm{ArH}), 7.65(1 \mathrm{H}, \mathrm{d}, J=2.0 \mathrm{~Hz}$, $\mathrm{ArH}), 7.68(1 \mathrm{H}, \mathrm{dd}, J=8.4,2.0 \mathrm{~Hz}, \mathrm{ArH}), 7.80(1 \mathrm{H}, \mathrm{d}, J=15.6 \mathrm{~Hz},-\mathrm{CH}=\mathrm{CHCO}-) .{ }^{13} \mathrm{C}-\mathrm{NMR}(500 \mathrm{MHz}$, $\left.\mathrm{CDCl}_{3}\right): \delta 55.4,56.1,56.6,76.4,77.8,111.3,112.3,114.4,119.3,122.3,127.7,130.1,132.6,144.0,149.7$, 
150.7, 161.6, 188.7. LCMS (ES+) $m / z=323.2\left([\mathrm{M}+\mathrm{H}]^{+}, t_{\mathrm{R}}=1.56 \mathrm{~min}\right)$. HRMS (ESI+) $m / z=323.1265$ $[\mathrm{M}+\mathrm{H}]^{+}$found, $\mathrm{C}_{20} \mathrm{H}_{19} \mathrm{O}_{4}{ }^{+}$required 323.1278.

(E)-1-(5-Bromo-2-(prop-2-yn-1-yloxy)phenyl)-3-(1-methyl-1H-indol-3-yl)prop-2-en-1-one (21). A mixture of indole chalcone $14(2.07 \mathrm{~g}, 5.81 \mathrm{mmol})$, propargyl bromide $(1.50 \mathrm{~mL}, 16.8 \mathrm{mmol})$ and anhydrous $\mathrm{K}_{2} \mathrm{CO}_{3}(2.52 \mathrm{~g}, 18.2 \mathrm{mmol})$ in dry acetone $(100 \mathrm{~mL})$ was reacted according to GP-C. The crude residue was purified by flash column chromatography $\left(\mathrm{SiO}_{2}, \mathrm{CH}_{2} \mathrm{Cl}_{2}\right)$ to afford chalcone $21(2.20 \mathrm{~g}$, 96\%) as a bright yellow powdery solid. m.p. $126-128{ }^{\circ} \mathrm{C}$. TLC $R_{\mathrm{f}}=0.20\left(\mathrm{CH}_{2} \mathrm{Cl}_{2}\right)$. IR $v_{\max }$ (neat) $/ \mathrm{cm}^{-1}$ : 3257w (C $\equiv C-H$ str), 3070w (C-H str), 2942w (C-H str), 2114w (C $\equiv C$ str), 1638s (C=O str), 1587m $(\mathrm{C}=\mathrm{C}$ str), 1544s ( $\mathrm{C}=\mathrm{C}$ str), 1524s (C=C str), 1473m, 1373s, 1269s, 1213m, 1176s, 1133m, 1073m, 1006s. ${ }^{1} \mathrm{H}-\mathrm{NMR}\left(500 \mathrm{MHz}, \mathrm{CDCl}_{3}\right): \delta 2.55\left(1 \mathrm{H}, \mathrm{t}, J=2.4 \mathrm{~Hz},-\mathrm{OCH}_{2} \mathrm{C} \equiv \mathrm{CH}\right), 3.82\left(3 \mathrm{H}, \mathrm{s},-\mathrm{NCH}_{3}\right), 4.79(2 \mathrm{H}, \mathrm{d}$, $\left.J=2.4 \mathrm{~Hz},-\mathrm{OCH}_{2} \mathrm{C} \equiv \mathrm{CH}\right), 7.03(1 \mathrm{H}, \mathrm{d}, J=9.2 \mathrm{~Hz}, \mathrm{ArH}), 7.26-7.38(3 \mathrm{H}, \mathrm{m}, \mathrm{ArH}), 7.38(1 \mathrm{H}, \mathrm{d}, J=16.0 \mathrm{~Hz}$, - $\mathrm{CH}=\mathrm{CHCO}-), 7.41(1 \mathrm{H}, \mathrm{s}, \mathrm{ArH}), 7.55(1 \mathrm{H}, \mathrm{dd}, J=8.8,2.4 \mathrm{~Hz}, \mathrm{ArH}), 7.77(1 \mathrm{H}, \mathrm{d}, J=2.4 \mathrm{~Hz}, \mathrm{ArH}), 7.88$ $(1 \mathrm{H}, \mathrm{d}, J=15.6 \mathrm{~Hz},-\mathrm{CH}=\mathrm{CHCO}-), 8.01(1 \mathrm{H}, \mathrm{d}, J=8.0 \mathrm{~Hz}, \mathrm{ArH}) .{ }^{13} \mathrm{C}-\mathrm{NMR}\left(500 \mathrm{MHz}, \mathrm{CDCl}_{3}\right): \delta 33.3$, 56.6, 76.4, 77.8, 110.0, 112.9, 114.3, 115.3, 121.0, 121.5, 121.6, 123.2, 126.0, 132.6, 132.9, 134.3, 134.8, 138.2, 138.6, 154.6, 191.0. LCMS (ES+) $m / z=396.1\left([\mathrm{M}+\mathrm{H}]^{+}, t_{\mathrm{R}}=1.98 \mathrm{~min}\right)$. HRMS (ESI+) $m / z=394.0425$ $[\mathrm{M}+\mathrm{H}]^{+}$found, $\mathrm{C}_{21} \mathrm{H}_{17} \mathrm{NO}_{2} \mathrm{Br}^{+}$required 394.0443.

(E)-1-(3-Methoxy-4-(prop-2-yn-1-yloxy)phenyl)-3-(1-methyl-1H-pyrrol-2-yl)prop-2-en-1-one (22). A mixture of pyrrole chalcone $16(1.00 \mathrm{~g}, 3.89 \mathrm{mmol})$, propargyl bromide $(0.70 \mathrm{~mL}, 7.86 \mathrm{mmol})$ and anhydrous $\mathrm{K}_{2} \mathrm{CO}_{3}(1.61 \mathrm{~g}, 1.17 \mathrm{mmol})$ in dry acetone $(50 \mathrm{~mL})$ was reacted according to GP-C. The crude residue was purified by flash column chromatography $\left(\mathrm{SiO}_{2}, \mathrm{PE} / \mathrm{EtOAc} ; 3: 1\right)$ to afford chalcone 22 (1.06 $\mathrm{g}$, $92 \%$ ) as a bright yellow powdery solid. m.p. $164-166^{\circ} \mathrm{C}$. TLC $R_{\mathrm{f}}=0.41$ (PE/EtOAc; 1:1). IR $v_{\max }$ (neat) $/ \mathrm{cm}^{-1}$ : 3234m (C $\equiv \mathrm{C}-\mathrm{H}$ str), 2995w (C-H str), 2866w (C-H str), 1739m (C=O str), 1645m, 1598s, $(\mathrm{C}=\mathrm{C}$ str), 1561s ( $\mathrm{C}=\mathrm{C}$ str), $1515 \mathrm{~m}$ ( $\mathrm{C}=\mathrm{C}$ str), 1483m, 1416w, 1374m, 1346s, $1272 \mathrm{~s}, 1179 \mathrm{~m}, 1129 \mathrm{~s}, 1087 \mathrm{w}$, $1042 \mathrm{~m}, 1012 \mathrm{~m} .{ }^{1} \mathrm{H}-\mathrm{NMR}\left(500 \mathrm{MHz}, \mathrm{CDCl}_{3}\right): \delta 2.56\left(1 \mathrm{H}, \mathrm{t}, J=2.5 \mathrm{~Hz},-\mathrm{OCH}_{2} \mathrm{C} \equiv \mathrm{CH}\right), 3.78(3 \mathrm{H}, \mathrm{s}$, $\left.-\mathrm{NCH}_{3}\right), 3.98\left(3 \mathrm{H}, \mathrm{s},-\mathrm{OCH}_{3}\right), 4.86\left(1 \mathrm{H}, \mathrm{d}, J=2.5 \mathrm{~Hz},-\mathrm{OCH}_{2} \mathrm{C} \equiv \mathrm{CH}\right), 6.23-6.24(1 \mathrm{H}, \mathrm{m}, \mathrm{ArH}), 6.82$ $(1 \mathrm{H}, \mathrm{t}, J=2.0 \mathrm{~Hz}, \mathrm{ArH}), 6.85(1 \mathrm{H}, \mathrm{dd}, J=4.0,1.0 \mathrm{~Hz}, \mathrm{ArH}), 7.10(1 \mathrm{H}, \mathrm{d}, J=8.0 \mathrm{~Hz}, \mathrm{ArH}), 7.32(1 \mathrm{H}, \mathrm{d}$, $J=15.0 \mathrm{~Hz},-\mathrm{CH}=\mathrm{CHCO}-), 7.64-7.67(2 \mathrm{H}, \mathrm{m}, \mathrm{ArH}), 7.82(1 \mathrm{H}, \mathrm{d}, J=15.0 \mathrm{~Hz},-\mathrm{CH}=\mathrm{CHCO}-) .{ }^{13} \mathrm{C}-\mathrm{NMR}$ $\left(500 \mathrm{MHz}, \mathrm{CDCl}_{3}\right): \delta 34.4,56.1,56.6,76.4,77.8,109.7,111.2,112.1,112.4,116.4,122.0,127.6,130.3$, 131.7, 132.9, 149.6, 150.5, 188.1. LCMS (ES+) $m / z=296.2\left([\mathrm{M}+\mathrm{H}]^{+}, t_{\mathrm{R}}=1.57 \mathrm{~min}\right)$. HRMS (ESI+) $m / z=294.1119[\mathrm{M}-\mathrm{H}]^{+}$found, $\mathrm{C}_{18} \mathrm{H}_{16} \mathrm{O}_{3} \mathrm{~N}^{+}$required 294.1125.

(E)-3-(1-Methyl-1H-pyrrol-2-yl)-1-(2-(prop-2-yn-1-yloxy)phenyl)prop-2-en-1-one (23). A mixture of pyrrole chalcone 15 (308 mg, $1.32 \mathrm{mmol})$, propargyl bromide $(0.25 \mathrm{~mL}, 2.81 \mathrm{mmol})$ and anhydrous $\mathrm{K}_{2} \mathrm{CO}_{3}$ (568 mg, $4.11 \mathrm{mmol})$ in dry acetone $(20 \mathrm{~mL})$ was reacted according to GP-C. The crude residue was purified by flash column chromatography $\left(\mathrm{SiO}_{2}, \mathrm{PE} / \mathrm{EtOAc} ; 3: 1\right)$ to afford chalcone 23 (352 mg, $98 \%$ ) as a bright yellow viscous oil. TLC $R_{\mathrm{f}}=0.34$ (PE/EtOAc; $\left.2: 1\right)$. IR $v_{\max }$ (neat) $/ \mathrm{cm}^{-1}: 3283 \mathrm{w}$ (C $\equiv \mathrm{C}-\mathrm{H}$ str), 3072w (C-H str), 2923w (C-H str), 2120w (C $\equiv \mathrm{C}$ str), 1698w (C=O str), 1647m, 1598s (C=C str), 1580s (C=C str), 1564s (C=C str), 1480s, 1450m, 1330m, 1287s, 1217s, 1113w, 1057m, 1018s. ${ }^{1} \mathrm{H}-\mathrm{NMR}$ $\left(500 \mathrm{MHz}, \mathrm{CDCl}_{3}\right): \delta 2.54\left(1 \mathrm{H}, \mathrm{t}, J=2.4 \mathrm{~Hz},-\mathrm{OCH}_{2} \mathrm{C} \equiv \mathrm{CH}\right), 3.74\left(3 \mathrm{H}, \mathrm{s},-\mathrm{NCH}_{3}\right), 4.79(2 \mathrm{H}, \mathrm{d}, J=2.4 \mathrm{~Hz}$, $\left.-\mathrm{OCH}_{2} \mathrm{C} \equiv \mathrm{CH}\right), 6.20-6.21(1 \mathrm{H}, \mathrm{m}, \mathrm{ArH}), 6.78-6.80(2 \mathrm{H}, \mathrm{m}, \mathrm{ArH}), 7.08-7.12(2 \mathrm{H}, \mathrm{m}, \mathrm{ArH}), 7.19(1 \mathrm{H}, \mathrm{d}$, $J=15.2 \mathrm{~Hz},-\mathrm{CH}=\mathrm{CHCO}-), 7.47(1 \mathrm{H}, \mathrm{t}, J=8.8 \mathrm{~Hz}, \mathrm{ArH}), 7.65(1 \mathrm{H}, \mathrm{d}, J=15.6 \mathrm{~Hz},-\mathrm{CH}=\mathrm{CHCO}-), 7.66$ $(1 \mathrm{H}, \mathrm{dd}, J=7.6,2.0 \mathrm{~Hz}, \mathrm{ArH}) .{ }^{13} \mathrm{C}-\mathrm{NMR}\left(500 \mathrm{MHz}, \mathrm{CDCl}_{3}\right): \delta 34.5,56.4,75.9,78.2,109.6,112.8,113.3$, $121.8,121.9,127.7,130.2,130.5,130.5,131.1,132.2,155.7,191.9$. LCMS (ES+) $m / z=266.1\left([\mathrm{M}+\mathrm{H}]^{+}\right.$, $t_{\mathrm{R}}=1.57 \mathrm{~min}$ ). HRMS (ESI+) $m / z=266.1183[\mathrm{M}+\mathrm{H}]^{+}$found, $\mathrm{C}_{17} \mathrm{H}_{16} \mathrm{NO}_{2}{ }^{+}$required 266.1181.

(E)-1-(5-Bromo-2-(prop-2-yn-1-yloxy)phenyl)-3-(furan-2-yl)prop-2-en-1-one (24). A mixture of furan chalcone 17 (2.02 g, $6.89 \mathrm{mmol})$, propargyl bromide $(1.30 \mathrm{~mL}, 14.6 \mathrm{mmol})$ and anhydrous $\mathrm{K}_{2} \mathrm{CO}_{3}$ $(2.90 \mathrm{~g}, 21.0 \mathrm{mmol})$ in dry acetone $(100 \mathrm{~mL})$ was reacted according to GP-C. The crude residue was purified by flash column chromatography $\left(\mathrm{SiO}_{2}, \mathrm{CH}_{2} \mathrm{Cl}_{2}\right)$ to afford chalcone $24(2.14 \mathrm{~g}$, 94\%) as a pale yellow-brown powdery solid. m.p. $82-84{ }^{\circ} \mathrm{C}$. TLC $R_{\mathrm{f}}=0.21\left(\mathrm{PE} / \mathrm{CH}_{2} \mathrm{Cl}_{2} ; 1: 1\right)$. IR $\gamma_{\max }$ (neat) $/ \mathrm{cm}^{-1}$ : 
3224m (C $\equiv \mathrm{C}-\mathrm{H}$ str), 3118w (C-H str), 2118w ( $\mathrm{C} \equiv \mathrm{C}$ str), 1660m ( $\mathrm{C}=\mathrm{O}$ str), 1600s (C=C str), 1586m $(\mathrm{C}=\mathrm{C}$ str) $, 1554 \mathrm{~m}(\mathrm{C}=\mathrm{C}$ str $), 1476 \mathrm{~m}, 1394 \mathrm{~m}, 1313 \mathrm{w}, 1276 \mathrm{~m}, 1211 \mathrm{~s}, 1180 \mathrm{~m}, 1131 \mathrm{~m}, 1079 \mathrm{w}, 1048 \mathrm{~m}$, 1012s. ${ }^{1} \mathrm{H}-\mathrm{NMR}\left(500 \mathrm{MHz}, \mathrm{CDCl}_{3}\right): \delta 2.54\left(1 \mathrm{H}, \mathrm{t}, J=2.4 \mathrm{~Hz},-\mathrm{OCH}_{2} \mathrm{C} \equiv \mathrm{CH}\right), 4.78(2 \mathrm{H}, \mathrm{d}, J=2.4 \mathrm{~Hz}$, $\left.-\mathrm{OCH}_{2} \mathrm{C} \equiv \mathrm{CH}\right), 6.50-6.51(1 \mathrm{H}, \mathrm{m}, \mathrm{ArH}), 6.70(1 \mathrm{H}, \mathrm{d}, J=3.6 \mathrm{~Hz}, \mathrm{ArH}), 7.03(1 \mathrm{H}, \mathrm{d}, J=8.8 \mathrm{~Hz}, \mathrm{ArH})$, $7.21(1 \mathrm{H}, \mathrm{d}, J=15.6 \mathrm{~Hz},-\mathrm{CH}=\mathrm{CHCO}-), 7.40(1 \mathrm{H}, \mathrm{d}, J=15.6 \mathrm{~Hz},-\mathrm{CH}=\mathrm{CHCO}-), 7.52(1 \mathrm{H}, \mathrm{d}, J=1.2 \mathrm{~Hz}$, $\mathrm{ArH}), 7.56(1 \mathrm{H}, \mathrm{dd}, J=8.8,2.4 \mathrm{~Hz}, \mathrm{ArH}), 7.73(1 \mathrm{H}, \mathrm{d}, J=2.4 \mathrm{~Hz}, \mathrm{ArH}) .{ }^{13} \mathrm{C}-\mathrm{NMR}\left(500 \mathrm{MHz}, \mathrm{CDCl}_{3}\right)$ : $\delta$ 56.6, 76.5, 77.6, 112.6, 114.3, 115.4, 116.2, 123.8, 130.4, 131.6, 133.0, 135.0, 145.1, 151.5, 154.8, 190.5 . LCMS (ES+) $m / z=333.0\left([\mathrm{M}+\mathrm{H}]^{+}, t_{\mathrm{R}}=1.89 \mathrm{~min}\right)$. HRMS (ESI +$) m / z=330.9973[\mathrm{M}+\mathrm{H}]^{+}$found, $\mathrm{C}_{16} \mathrm{H}_{12} \mathrm{O}_{3} \mathrm{Br}^{+}$required 330.9970 .

(E)-3-(Ferrocenyl)-1-(3-methoxy-4-(prop-2-yn-1-yloxy)phenyl)prop-2-en-1-one (25). A mixture of ferrocene chalcone 19 (1.01 g, $2.79 \mathrm{mmol})$, propargyl bromide $(0.50 \mathrm{~mL}, 5.61 \mathrm{mmol})$ and anhydrous $\mathrm{K}_{2} \mathrm{CO}_{3}$ $(1.18 \mathrm{~g}, 8.56 \mathrm{mmol})$ in dry acetone $(50 \mathrm{~mL})$ was reacted according to GP-C. The crude residue was purified by flash column chromatography $\left(\mathrm{SiO}_{2}, \mathrm{CH}_{2} \mathrm{Cl}_{2}\right)$ to afford chalcone $25(1.01 \mathrm{~g}, 91 \%)$ as a dark red-purple microcrystalline solid. m.p. $128-130{ }^{\circ} \mathrm{C}$. TLC $R_{\mathrm{f}}=0.47\left(0.5 \% \mathrm{MeOH} / \mathrm{CH}_{2} \mathrm{Cl}_{2}\right)$. IR $v_{\max }$

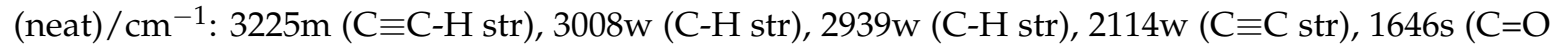
str), 1595m (C=C str), 1567s (C=C str), 1506m (C=C str), 1456m, 1413s, 1345w, 1295s, 1259s, 1194m, $1159 \mathrm{~s}, 1136 \mathrm{~s}, 1107 \mathrm{~m}, 1060 \mathrm{w}, 1044 \mathrm{~m}, 1020 \mathrm{~s} .{ }^{1} \mathrm{H}-\mathrm{NMR}\left(500 \mathrm{MHz}, \mathrm{CDCl}_{3}\right): \delta 2.56(1 \mathrm{H}, \mathrm{t}, J=2.5 \mathrm{~Hz}$, $\left.-\mathrm{OCH}_{2} \mathrm{C} \equiv \mathrm{CH}\right), 3.96\left(3 \mathrm{H}, \mathrm{s},-\mathrm{OCH}_{3}\right), 4.18\left(5 \mathrm{H}, \mathrm{s},-\mathrm{C}_{5} \mathrm{H}_{5}\right), 4.48\left(2 \mathrm{H}, \mathrm{t}, J=2.0 \mathrm{~Hz},-\mathrm{C}_{5} \mathrm{H}_{4}\right), 4.60(2 \mathrm{H}, \mathrm{t}$, $\left.J=2.0 \mathrm{~Hz},-\mathrm{C}_{5} \mathrm{H}_{4}\right), 4.85\left(2 \mathrm{H}, \mathrm{d}, J=2.0 \mathrm{~Hz},-\mathrm{OCH}_{2} \mathrm{C} \equiv \mathrm{CH}\right), 7.09(1 \mathrm{H}, \mathrm{d}, J=8.5 \mathrm{~Hz}, \mathrm{ArH}), 7.15(1 \mathrm{H}, \mathrm{d}$, $J=15.5 \mathrm{~Hz},-\mathrm{CH}=\mathrm{CHCO}-), 7.61-7.63(2 \mathrm{H}, \mathrm{m}, \mathrm{ArH}), 7.75(1 \mathrm{H}, \mathrm{d}, J=15.0 \mathrm{~Hz},-\mathrm{CH}=\mathrm{CHCO}-) .{ }^{13} \mathrm{C}-\mathrm{NMR}$ $\left(500 \mathrm{MHz}, \mathrm{CDCl}_{3}\right): \delta 56.0,56.5,68.9,69.7,71.2,76.3,77.8,79.3,111.2,112.3,118.5,122.1,132.6,146.0$, 149.5, 150.4, 187.9. LCMS (ES+) $m / z=401.1\left([\mathrm{M}+\mathrm{H}]^{+}, t_{\mathrm{R}}=1.81 \mathrm{~min}\right)$. HRMS (ESI+) $m / z=401.0823$ $[\mathrm{M}+\mathrm{H}]^{+}$found, $\mathrm{C}_{23} \mathrm{H}_{21} \mathrm{O}_{3} \mathrm{Fe}^{+}$required 401.0840 .

(E)-3-(Ferrocenyl)-1-(2-(prop-2-yn-1-yloxy)phenyl)prop-2-en-1-one (26). A mixture of chalcone 18 (1.02 g, $3.07 \mathrm{mmol})$, propargyl bromide $(0.54 \mathrm{~mL}, 6.02 \mathrm{mmol})$ and anhydrous $\mathrm{K}_{2} \mathrm{CO}_{3}(1.20 \mathrm{~g}, 8.68 \mathrm{mmol})$ in dry acetone $(50 \mathrm{~mL})$ was reacted according to GP-C. The crude residue was purified by flash column chromatography $\left(\mathrm{SiO}_{2}, \mathrm{PE} / \mathrm{CH}_{2} \mathrm{Cl}_{2} ; 1: 1\right)$ to afford chalcone $\mathbf{2 6}(919 \mathrm{mg}, 81 \%)$ as a bright red powdery solid. m.p. $90-92{ }^{\circ} \mathrm{C}$. TLC $R_{\mathrm{f}}=0.38\left(\mathrm{CH}_{2} \mathrm{Cl}_{2}\right)$. IR $v_{\max }$ (neat) $/ \mathrm{cm}^{-1}: 3257 \mathrm{~m}(\mathrm{C} \equiv \mathrm{C}-\mathrm{H}$ str), $2924 \mathrm{w}$ (C-H str), 2850w (C-H str), 2117w (C $\equiv \mathrm{C}$ str), 1654s (C=O str), 1587s (C=C str), 1480m, 1448m, 1356w, 1345w, 1289m, 1211s, 1159w, 1104m, 1058m, 1016s, 1001s. ${ }^{1} \mathrm{H}-\mathrm{NMR}\left(500 \mathrm{MHz}, \mathrm{CDCl}_{3}\right): \delta 2.56(1 \mathrm{H}, \mathrm{t}$, $\left.J=2.5 \mathrm{~Hz},-\mathrm{OCH}_{2} \mathrm{C} \equiv \mathrm{CH}\right), 4.19\left(5 \mathrm{H}, \mathrm{s},-\mathrm{C}_{5} \mathrm{H}_{5}\right), 4.46\left(2 \mathrm{H}, \mathrm{t}, J=2.0 \mathrm{~Hz},-\mathrm{C}_{5} \mathrm{H}_{4}\right), 4.56(2 \mathrm{H}, \mathrm{t}, J=2.0 \mathrm{~Hz}$, $\left.-\mathrm{C}_{5} \mathrm{H}_{4}\right), 4.79\left(2 \mathrm{H}, \mathrm{d}, J=2.5 \mathrm{~Hz},-\mathrm{OCH}_{2} \mathrm{C} \equiv \mathrm{CH}\right), 6.95(1 \mathrm{H}, \mathrm{d}, J=16.0 \mathrm{~Hz},-\mathrm{CH}=\mathrm{CHCO}-), 7.09-7.13(2 \mathrm{H}$, $\mathrm{m}, \mathrm{ArH}), 7.47(1 \mathrm{H}, \mathrm{t}, J=9.0 \mathrm{~Hz}, \mathrm{ArH}), 7.50(1 \mathrm{H}, \mathrm{d}, J=15.5 \mathrm{~Hz},-\mathrm{CH}=\mathrm{CHCO}-), 7.58(1 \mathrm{H}, \mathrm{dd}, J=7.5$, $1.5 \mathrm{~Hz}, \mathrm{ArH}) .{ }^{13} \mathrm{C}-\mathrm{NMR}\left(500 \mathrm{MHz}, \mathrm{CDCl}_{3}\right): \delta 56.3,69.0,69.8,71.2,76.0,78.2,79.0,113.3,121.7,124.6$, $130.1,130.5,131.9,146.2,155.4,192.5$. LCMS (ES+) $m / z=371.1\left([\mathrm{M}+\mathrm{H}]^{+}, t_{\mathrm{R}}=1.96 \mathrm{~min}\right)$. HRMS (ESI+) $m / z=370.0639[\mathrm{M}]^{+}$found, $\mathrm{C}_{22} \mathrm{H}_{18} \mathrm{O}_{2} \mathrm{Fe}^{+}$required 370.0651.

3-(Prop-2-yn-1-yloxy)flavone (30). A mixture of 3-hydroxyflavone 27 (2.06 g, $8.65 \mathrm{mmol}$ ), propargyl bromide $(1.50 \mathrm{~mL}, 16.8 \mathrm{mmol})$ and anhydrous $\mathrm{K}_{2} \mathrm{CO}_{3}(3.48 \mathrm{~g}, 25.2 \mathrm{mmol})$ in dry acetone $(100 \mathrm{~mL})$ was reacted according to GP-C. The crude residue was purified by flash column chromatography $\left(\mathrm{SiO}_{2}\right.$, $\left.\mathrm{CH}_{2} \mathrm{Cl}_{2}\right)$ to afford flavone $30(2.38 \mathrm{~g}, 99 \%)$ as a pale yellow-white powdery solid. m.p. $104-106^{\circ} \mathrm{C}$. TLC $R_{\mathrm{f}}=0.24\left(\mathrm{CH}_{2} \mathrm{Cl}_{2}\right)$. IR $v_{\max }$ (neat) $/ \mathrm{cm}^{-1}: 3254 \mathrm{~m}$ (C $\equiv \mathrm{C}-\mathrm{H}$ str), 2946w (C-H str), 2114w (C $\equiv \mathrm{C}$ str), 1612s (C=O str), 1600s, 1557s (C=C str), 1468s, 1445m, 1399s, 1354m, 1345m, 1279w, 1234w, 1195s, 1185s, $1148 \mathrm{~s}, 1110 \mathrm{~m}, 1079 \mathrm{w}, 1036 \mathrm{~m} .{ }^{1} \mathrm{H}-\mathrm{NMR}\left(500 \mathrm{MHz}, \mathrm{CDCl}_{3}\right): \delta 2.33\left(1 \mathrm{H}, \mathrm{t}, J=2.4 \mathrm{~Hz},-\mathrm{OCH}_{2} \mathrm{C} \equiv \mathrm{CH}\right), 5.00$ $\left(2 \mathrm{H}, \mathrm{d}, J=2.4 \mathrm{~Hz},-\mathrm{OCH}_{2} \mathrm{C} \equiv \mathrm{CH}\right), 7.42(1 \mathrm{H}, \mathrm{t}, J=8.0 \mathrm{~Hz}, \mathrm{ArH}), 7.50-7.54(3 \mathrm{H}, \mathrm{m}, \mathrm{ArH}), 7.56(1 \mathrm{H}, \mathrm{d}$, $J=8.4 \mathrm{~Hz}, \mathrm{ArH}), 7.70(1 \mathrm{H}, \mathrm{t}, J=8.4 \mathrm{~Hz}, \mathrm{ArH}), 8.14-8.16(2 \mathrm{H}, \mathrm{m}, \mathrm{ArH}), 8.27(1 \mathrm{H}, \mathrm{dd}, J=8.0,1.6 \mathrm{~Hz}, \mathrm{ArH})$. ${ }^{13} \mathrm{C}-\mathrm{NMR}\left(500 \mathrm{MHz}, \mathrm{CDCl}_{3}\right): \delta 59.1,76.1,78.5,118.0,123.9,124.7,125.7,128.3,128.9,130.7,130.9,133.5$, $138.5,155.2,156.7,174.8$. LCMS (ES+) $m / z=277.5\left([\mathrm{M}+\mathrm{H}]^{+}, t_{\mathrm{R}}=4.47 \mathrm{~min}\right)$. These characterisation data are in accordance with that previously reported in the literature [23]. 
6-(Prop-2-yn-1-yloxy)flavone (31). A mixture of 6-hydroxyflavone 28 (2.00 g, $8.39 \mathrm{mmol})$, propargyl bromide $(1.50 \mathrm{~mL}, 16.8 \mathrm{mmol})$ and anhydrous $\mathrm{K}_{2} \mathrm{CO}_{3}(3.50 \mathrm{~g}, 25.3 \mathrm{mmol})$ in dry acetone $(100 \mathrm{~mL}) \mathrm{was}$ reacted according to GP-C. The crude residue was purified by flash column chromatography $\left(\mathrm{SiO}_{2}\right.$, $\left.1 \% \mathrm{MeOH} / \mathrm{CH}_{2} \mathrm{Cl}_{2}\right)$ to afford flavone $31(2.03 \mathrm{~g}, 87 \%)$ as a white crystalline solid. m.p. $170-172{ }^{\circ} \mathrm{C}$. TLC $R_{\mathrm{f}}=0.44\left(1 \% \mathrm{MeOH} / \mathrm{CH}_{2} \mathrm{Cl}_{2}\right)$. IR $v_{\max }$ (neat) $/ \mathrm{cm}^{-1}: 3266 \mathrm{~m}(\mathrm{C} \equiv \mathrm{C}-\mathrm{H} \mathrm{str}), 3059 \mathrm{w}(\mathrm{C}-\mathrm{H} \mathrm{str})$, 2116w (C $\equiv \mathrm{C}$ str), 1623s (C=O str), 1606s, 1582s (C=C str), 1568s (C=C str), 1497m, 1482m, 1455s, 1365s, $1283 \mathrm{~m}, 1259 \mathrm{~m}, 1234 \mathrm{~m}, 1186 \mathrm{~s}, 1144 \mathrm{~s}, 1075 \mathrm{~m}, 1048 \mathrm{~m}, 1007 \mathrm{~s} .{ }^{1} \mathrm{H}-\mathrm{NMR}\left(500 \mathrm{MHz}, \mathrm{CDCl}_{3}\right): \delta 2.57(1 \mathrm{H}, \mathrm{t}$, $\left.J=2.4 \mathrm{~Hz},-\mathrm{OCH}_{2} \mathrm{C} \equiv \mathrm{CH}\right), 4.76\left(2 \mathrm{H}, \mathrm{d}, J=2.4 \mathrm{~Hz},-\mathrm{OCH}_{2} \mathrm{C} \equiv \mathrm{CH}\right), 6.77(1 \mathrm{H}, \mathrm{s},-\mathrm{C}=\mathrm{CH}), 7.31(1 \mathrm{H}, \mathrm{dd}$, $J=9.2,3.2 \mathrm{~Hz}, \mathrm{ArH}), 7.46-7.51(4 \mathrm{H}, \mathrm{m}, \mathrm{ArH}), 7.65(1 \mathrm{H}, \mathrm{d}, J=3.2 \mathrm{~Hz}, \mathrm{ArH}), 7.86-7.88(2 \mathrm{H}, \mathrm{m}, \mathrm{ArH})$. ${ }^{13} \mathrm{C}-\mathrm{NMR}\left(500 \mathrm{MHz}, \mathrm{CDCl}_{3}\right): \delta 56.3,76.0,77.7,106.4,106.7,119.6,123.9,124.4,126.1,128.9,131.4,131.6$, $151.3,154.7,163.0,177.9$. LCMS (ES+) $m / z=277.0\left([\mathrm{M}+\mathrm{H}]^{+}, t_{\mathrm{R}}=1.56 \mathrm{~min}\right)$. These characterisation data are in accordance with that previously reported in the literature [24].

7-(Prop-2-yn-1-yloxy)flavone (32). A mixture of 7-hydroxyflavone 29 (990 mg, $4.16 \mathrm{mmol}$ ), propargyl bromide $(0.748 \mathrm{~mL}, 8.39 \mathrm{mmol})$ and anhydrous $\mathrm{K}_{2} \mathrm{CO}_{3}(1.75 \mathrm{~g}, 12.7 \mathrm{mmol})$ and in dry acetone $(100 \mathrm{~mL})$ was reacted according to GP-C. The crude residue was purified by flash column chromatography $\left(\mathrm{SiO}_{2}\right.$, $\left.1 \% \mathrm{MeOH} / \mathrm{CH}_{2} \mathrm{Cl}_{2}\right)$ to afford flavone $32(1.10 \mathrm{~g}, 95 \%)$ as an off-white powdery solid. m.p. $216-218{ }^{\circ} \mathrm{C}$. TLC $R_{\mathrm{f}}=0.44\left(2 \% \mathrm{MeOH} / \mathrm{CH}_{2} \mathrm{Cl}_{2}\right)$. IR $v_{\max }$ (neat) $/ \mathrm{cm}^{-1}: 3194 \mathrm{w}(\mathrm{C} \equiv \mathrm{C}-\mathrm{H}$ str), 2921w $(\mathrm{C}-\mathrm{H}$ str), $2115 \mathrm{w}$ (C $\equiv \mathrm{C}$ str), 1619s (C=O str), 1591s (C=C str), 1568s (C=C str), 1496m, 1451s, 1435s, 1378s, 1350m, 1268m, $1229 \mathrm{~m}, 1168 \mathrm{~s}, 1135 \mathrm{~s}, 1091 \mathrm{~s}, 1017 \mathrm{~s} .{ }^{1} \mathrm{H}-\mathrm{NMR}\left(500 \mathrm{MHz}, \mathrm{CDCl}_{3}\right): \delta 2.62\left(1 \mathrm{H}, \mathrm{t}, J=2.4 \mathrm{~Hz},-\mathrm{OCH}_{2} \mathrm{C} \equiv \mathrm{CH}\right)$, $4.83\left(2 \mathrm{H}, \mathrm{d}, J=2.4 \mathrm{~Hz},-\mathrm{OCH}_{2} \mathrm{C} \equiv \mathrm{CH}\right), 6.77(1 \mathrm{H}, \mathrm{s},-\mathrm{C}=\mathrm{CH}), 7.05(1 \mathrm{H}, \mathrm{dd}, J=8.8,2.4 \mathrm{~Hz}, \mathrm{ArH}), 7.09$ $(1 \mathrm{H}, \mathrm{d}, J=2.4 \mathrm{~Hz}, \mathrm{ArH}), 7.51-7.55$ (3H, m, ArH), 7.90-7.93 (2H, m, ArH), $8.17(1 \mathrm{H}, \mathrm{d}, J=8.8 \mathrm{~Hz}, \mathrm{ArH})$. ${ }^{13} \mathrm{C}-\mathrm{NMR}\left(500 \mathrm{MHz}, \mathrm{CDCl}_{3}\right): \delta 56.2,76.6,77.3,101.8,107.6,114.7,118.4,126.2,127.2,129.0,131.5,131.8$, $157.7,161.9,163.1,177.7$. LCMS $(\mathrm{ES}+) \mathrm{m} / z=277.0\left([\mathrm{M}+\mathrm{H}]^{+}, t_{\mathrm{R}}=1.54 \mathrm{~min}\right)$. These characterisation data are in accordance with that previously reported in the literature [24].

4-(Prop-2-yn-1-yloxy)benzaldehyde (34). A mixture of benzaldehyde 33 (10.2 g, $83.7 \mathrm{mmol})$, propargyl bromide $(14.6 \mathrm{~mL}, 164 \mathrm{mmol})$ and anhydrous $\mathrm{K}_{2} \mathrm{CO}_{3}(22.7 \mathrm{~g}, 165 \mathrm{mmol})$ in dry acetone $(200 \mathrm{~mL})$ was reacted according to GP-C. The crude residue was purified by flash column chromatography $\left(\mathrm{SiO}_{2}, \mathrm{CH}_{2} \mathrm{Cl}_{2}\right)$ to afford benzaldehyde $34(12.2 \mathrm{~g}, 91 \%)$ as a white crystalline solid. m.p. $88-90{ }^{\circ} \mathrm{C}$. TLC $R_{\mathrm{f}}=0.49\left(\mathrm{CH}_{2} \mathrm{Cl}_{2}\right)$. IR $v_{\max }$ (neat) $/ \mathrm{cm}^{-1}$ : 3205m (C $\equiv \mathrm{C}-\mathrm{H}$ str), 2971w (C-H str), 2837w (C-H str), 2123w (C $\equiv \mathrm{C}$ str), 1739s (C=O str), 1679s, 1601s, 1576s (C=C str), 1508m (C=C str), 1428w, 1384s, 1245s, 1217s, 1170s, 1009s. ${ }^{1} \mathrm{H}-\mathrm{NMR}\left(500 \mathrm{MHz} \mathrm{CDCl}_{3}\right): \delta 2.58\left(1 \mathrm{H}, \mathrm{t}, J=2.4 \mathrm{~Hz},-\mathrm{OCH}_{2} \mathrm{C} \equiv \mathrm{CH}\right), 4.77(2 \mathrm{H}$, $\left.\mathrm{d}, J=2.4 \mathrm{~Hz},-\mathrm{OCH}_{2} \mathrm{C} \equiv \mathrm{CH}\right), 7.08(2 \mathrm{H}, \mathrm{d}, J=8.8 \mathrm{~Hz}, \mathrm{ArH}), 7.85(2 \mathrm{H}, \mathrm{d}, J=8.8 \mathrm{~Hz}, \mathrm{ArH}), 9.89(1 \mathrm{H}, \mathrm{s}$, CHO). ${ }^{13} \mathrm{C}-\mathrm{NMR}\left(500 \mathrm{MHz}, \mathrm{CDCl}_{3}\right): \delta 55.9,76.3,77.5,115.1,130.5,131.8,162.3,190.7$. LCMS (ES+) $\mathrm{m} / z$ $=161.1\left([\mathrm{M}+\mathrm{H}]^{+}, t_{\mathrm{R}}=1.65 \mathrm{~min}\right)$. These characterisation data are in accordance with that previously reported in the literature [41].

(E)-1-(2-Hydroxyphenyl)-3-(4-(prop-2-yn-1-yloxy)phenyl)prop-2-en-1-one (35). A mixture of benzaldehyde $34(5.04 \mathrm{~g}, 31.5 \mathrm{mmol})$, acetophenone 10 (3.76 mL, $31.2 \mathrm{mmol})$, and $\mathrm{KOH}(10.6 \mathrm{~g}, 189 \mathrm{mmol})$ in absolute $\mathrm{EtOH}(100 \mathrm{~mL})$ was reacted according to GP-D. The crude residue was purified by recrystallization from $\mathrm{MeOH}$ to afford chalcone $35(6.67 \mathrm{~g}, 77 \%)$ as a bright yellow powdery solid. m.p. $144-146{ }^{\circ} \mathrm{C}$. TLC $R_{\mathrm{f}}=0.45$ (PE/EtOAc; 3:1). IR $v_{\max }$ (neat) $/ \mathrm{cm}^{-1}: 3487 \mathrm{w}(\mathrm{O}-\mathrm{H}$ str), 3240m (C $\equiv \mathrm{C}-\mathrm{H}$ str), $2923 \mathrm{w}$ (C-H str), 2124w (C $\equiv$ C str), 1634s (C=O str), 1604m, 1559s (C=C str), 1509s (C=C str), 1488s, 1442m, $1368 \mathrm{~m}, 1299 \mathrm{~s}, 1270 \mathrm{~m}, 1203 \mathrm{~m}, 1177 \mathrm{~s}, 1159 \mathrm{~s}, 1027 \mathrm{~s} .{ }^{1} \mathrm{H}-\mathrm{NMR}\left(500 \mathrm{MHz}, \mathrm{CDCl}_{3}\right): \delta 2.58(1 \mathrm{H}, \mathrm{t}, J=2.4 \mathrm{~Hz}$, $\left.-\mathrm{OCH}_{2} \mathrm{C} \equiv \mathrm{CH}\right), 4.75\left(2 \mathrm{H}, \mathrm{d}, J=2.4 \mathrm{~Hz},-\mathrm{OCH}{ }_{2} \mathrm{C} \equiv \mathrm{CH}\right), 6.94(1 \mathrm{H}, \mathrm{t}, J=7.6 \mathrm{~Hz}, \mathrm{ArH}), 7.03(3 \mathrm{H}, \mathrm{d}$, $J=8.4 \mathrm{~Hz}, \mathrm{ArH}), 7.49(1 \mathrm{H}, \mathrm{t}, J=8.4 \mathrm{~Hz}, \mathrm{ArH}), 7.54(1 \mathrm{H}, \mathrm{d}, J=15.2 \mathrm{~Hz},-\mathrm{CH}=\mathrm{CHCO}-), 7.63(2 \mathrm{H}, \mathrm{d}$, $J=8.8 \mathrm{~Hz}, \mathrm{ArH}), 7.89(1 \mathrm{H}, \mathrm{d}, J=15.2 \mathrm{~Hz},-\mathrm{CH}=\mathrm{CHCO}-), 7.90-7.92(1 \mathrm{H}, \mathrm{m}, \mathrm{ArH}), 12.93(1 \mathrm{H}, \mathrm{s}, \mathrm{OH})$. ${ }^{13} \mathrm{C}-\mathrm{NMR}\left(500 \mathrm{MHz}, \mathrm{CDCl}_{3}\right): \delta 55.8,76.1,77.9,115.3,118.0,118.5,118.7,120.0,128.0,129.5,130.4$, $136.1,145.0,159.7,163.5,193.5$. LCMS (ES+) $m / z=279.1\left([\mathrm{M}+\mathrm{H}]^{+}, t_{\mathrm{R}}=1.75 \mathrm{~min}\right)$. HRMS (ESI+) $m / z=279.1005[\mathrm{M}+\mathrm{H}]^{+}$found, $\mathrm{C}_{18} \mathrm{H}_{15} \mathrm{O}_{3}{ }^{+}$required 279.1016. 
3-Hydroxy-4'-(prop-2-yn-1-yloxy)flavone (36). A mixture of chalcone 35 (2.02 g, $7.26 \mathrm{mmol}), 16 \% \mathrm{NaOH}$ (14.4 mL) and $15 \% \mathrm{H}_{2} \mathrm{O}_{2}(7.19 \mathrm{~mL})$ in $\mathrm{MeOH}(50 \mathrm{~mL})$ was reacted according to GP-F. The crude residue was purified by flash column chromatography $\left(\mathrm{SiO}_{2}, 1 \% \mathrm{MeOH} / \mathrm{CH}_{2} \mathrm{Cl}_{2}\right)$ to afford flavonol $36(1.56 \mathrm{~g}$, $73 \%)$ as a pale yellow-white powdery solid. m.p. $194-196{ }^{\circ} \mathrm{C}$. TLC $R_{\mathrm{f}}=0.44\left(0.5 \% \mathrm{MeOH} / \mathrm{CH}_{2} \mathrm{Cl}_{2}\right)$. IR $v_{\max }$ (neat) $/ \mathrm{cm}^{-1}: 3271 \mathrm{~s}$ (C $\equiv \mathrm{C}-\mathrm{H}$ str), 3260w (O-H str), 2979w (C-H str), 2119w (C $\equiv \mathrm{C}$ str), $1599 \mathrm{~s}$ (C=O str), 1562s (C=C str), 1507m (C=C str), 1483m, 1470m, 1427m, 1411m, 1233s, 1216m, 1180s, 1120s, 1109s, 1017s. ${ }^{1} \mathrm{H}-\mathrm{NMR}\left(500 \mathrm{MHz}, \mathrm{CDCl}_{3}\right): \delta 2.58\left(1 \mathrm{H}, \mathrm{t}, J=2.4 \mathrm{~Hz},-\mathrm{OCH}_{2} \mathrm{C} \equiv \mathrm{CH}\right), 4.79(2 \mathrm{H}, \mathrm{d}, J=2.4 \mathrm{~Hz}$, $\left.-\mathrm{OCH}_{2} \mathrm{C} \equiv \mathrm{CH}\right), 7.00(1 \mathrm{H}, \mathrm{br} \mathrm{s}, \mathrm{OH}), 7.14(2 \mathrm{H}, \mathrm{d}, J=9.2 \mathrm{~Hz}, \mathrm{ArH}), 7.42(1 \mathrm{H}, \mathrm{t}, J=8.0 \mathrm{~Hz}, \mathrm{ArH}), 7.58$ $(1 \mathrm{H}, \mathrm{d}, J=8.4 \mathrm{~Hz}, \mathrm{ArH}), 7.70(1 \mathrm{H}, \mathrm{t}, J=8.4 \mathrm{~Hz}, \mathrm{ArH}), 8.25-8.28(3 \mathrm{H}, \mathrm{m}, \mathrm{ArH}) .{ }^{13} \mathrm{C}-\mathrm{NMR}(500 \mathrm{MHz}$, $\left.\mathrm{CDCl}_{3}\right): \delta 55.8,76.0,78.0,115.0,118.2,120.7,124.4,124.4,125.4,129.5,133.4,137.8,145.0,155.3,158.9$, 173.2. LCMS (ES+) $m / z=293.1\left(\left[\mathrm{M}+\mathrm{H}^{+}, t_{\mathrm{R}}=1.62 \mathrm{~min}\right)\right.$. HRMS (ESI+) $m / z=315.0618[\mathrm{M}+\mathrm{Na}]^{+}$ found, $\mathrm{C}_{18} \mathrm{H}_{12} \mathrm{O}_{4} \mathrm{Na}^{+}$required 315.0628 .

1-(2-Hydroxy-4-methoxyphenyl)-2-(4-hydroxyphenyl)ethan-1-one (41). A mixture of phenol 37 (8.70 mL, $79.32 \mathrm{mmol})$ and phenylacetic acid $39(10.1 \mathrm{~g}, 66.3 \mathrm{mmol})$ in $\mathrm{BF}_{3} \cdot \mathrm{OEt}_{2}(85.0 \mathrm{~mL}, 677 \mathrm{mmol})$ was reacted according to GP-G. The crude residue was purified by flash column chromatography $\left(\mathrm{SiO}_{2}, \mathrm{CH}_{2} \mathrm{Cl}_{2}\right)$ to afford phenylethanone $41(13.2 \mathrm{~g}, 78 \%)$ as a white powdery solid. m.p. $158-160{ }^{\circ} \mathrm{C}$. TLC $R_{\mathrm{f}}=0.38$ $\left(1 \% \mathrm{MeOH} / \mathrm{CH}_{2} \mathrm{Cl}_{2}\right)$. IR $v_{\max }$ (neat) $/ \mathrm{cm}^{-1}$ : 3469w(br) (O-H str), 3221w(br ) (O-H str), 2984w (C-H str), 2904w (C-H str), 1706w (C=O str), 1613s (C=C str), 1518s (C=C str), 1450m, 1437s, 1381m, 1354s, 1291s, 1224s, 1202s, 1127s, 1021m. ${ }^{1} \mathrm{H}-\mathrm{NMR}\left(500 \mathrm{MHz},\left(\mathrm{CD}_{3}\right)_{2} \mathrm{CO}\right): \delta 3.85\left(3 \mathrm{H}, \mathrm{s},-\mathrm{OCH}_{3}\right), 4.19(2 \mathrm{H}$, $\left.\mathrm{s},-\mathrm{COCH}_{2}\right), 6.42(1 \mathrm{H}, \mathrm{d}, J=2.8 \mathrm{~Hz}, \mathrm{ArH}), 6.49(1 \mathrm{H}, \mathrm{dd}, J=8.8,2.4 \mathrm{~Hz}, \mathrm{ArH}), 6.80(2 \mathrm{H}, \mathrm{d}, J=8.8 \mathrm{~Hz}$, $\mathrm{ArH}), 7.16(2 \mathrm{H}, \mathrm{d}, J=8.4 \mathrm{~Hz}, \mathrm{ArH}), 7.98(1 \mathrm{H}, \mathrm{d}, J=9.2 \mathrm{~Hz}, \mathrm{ArH}), 8.29(1 \mathrm{H}, \mathrm{br} \mathrm{s}, \mathrm{OH}), 12.80(1 \mathrm{H}, \mathrm{s}, \mathrm{OH})$. ${ }^{13} \mathrm{C}-\mathrm{NMR}\left(500 \mathrm{MHz},\left(\mathrm{CD}_{3}\right){ }_{2} \mathrm{CO}\right): \delta 43.7,55.4,101.0,107.5,113.2,115.6,125.9,130.6,133.1,156.6,166.0$, 166.5, 203.5. LCMS (ES+) $m / z=259.1\left([\mathrm{M}+\mathrm{H}]^{+}, t_{\mathrm{R}}=1.47 \mathrm{~min}\right)$. These characterisation data are in accordance with that previously reported in the literature [42].

1-(2,4-Dihydroxyphenyl)-2-(4-methoxyphenyl)ethan-1-one (42). A mixture of phenol 38 (8.00 g, $72.7 \mathrm{mmol})$ and phenylacetic acid $40(10.2 \mathrm{~g}, 61.5 \mathrm{mmol})$ in $\mathrm{BF}_{3} \cdot \mathrm{OEt}_{2}(70.0 \mathrm{~mL}, 557 \mathrm{mmol})$ was reacted according to GP-G. The crude residue was purified by recrystallization from $\mathrm{CHCl}_{3}$ to afford phenylethanone $42(5.67 \mathrm{~g}, 36 \%)$ as a pale yellow-white powdery solid. m.p. $168-170{ }^{\circ} \mathrm{C}$. TLC $R_{\mathrm{f}}=0.50(1 \%$ $\mathrm{MeOH} / \mathrm{CH}_{2} \mathrm{Cl}_{2}$ ). IR $v_{\max }$ (neat) $/ \mathrm{cm}^{-1}: 3349 \mathrm{~s}$ (br) (O-H str), 2917w (C-H str), 2838w (C-H str), 1614s (C=O str), 1607s ( $C=C$ str), 1590s ( $C=C$ str), 1510s ( $C=C$ str), 1500m ( $=C$ str), 1436m, 1412w, 1351s, $1299 \mathrm{~m}, 1239 \mathrm{~m}, 1174 \mathrm{~s}, 1129 \mathrm{~m}, 1105 \mathrm{w}, 1023 \mathrm{~s} .{ }^{1} \mathrm{H}-\mathrm{NMR}\left(500 \mathrm{MHz},\left(\mathrm{CD}_{3}\right)_{2} \mathrm{CO}\right): \delta 3.76\left(3 \mathrm{H}, \mathrm{s},-\mathrm{OCH}_{3}\right)$, $4.22\left(2 \mathrm{H}, \mathrm{s},-\mathrm{COCH}_{2}\right), 6.33(1 \mathrm{H}, \mathrm{d}, J=2.0 \mathrm{~Hz}, \mathrm{ArH}), 6.44(1 \mathrm{H}, \mathrm{dd}, J=9.2,2.4 \mathrm{~Hz}, \mathrm{ArH}), 6.88(2 \mathrm{H}, \mathrm{d}$, $J=8.8 \mathrm{~Hz}, \mathrm{ArH}), 7.26(2 \mathrm{H}, \mathrm{d}, J=8.8 \mathrm{~Hz}, \mathrm{ArH}), 7.96(1 \mathrm{H}, \mathrm{d}, J=8.8 \mathrm{~Hz}, \mathrm{ArH}), 9.46(1 \mathrm{H}, \mathrm{s}, \mathrm{OH}), 12.73(1 \mathrm{H}$, s, OH). ${ }^{13} \mathrm{C}-\mathrm{NMR}\left(500 \mathrm{MHz},\left(\mathrm{CD}_{3}\right)_{2} \mathrm{CO}\right): \delta 43.6,54.8,103.0,108.2,112.8,114.1,127.3,130.7,133.7,159.0$, 165.0, 166.1, 203.1. LCMS (ES+) $m / z=259.1\left([\mathrm{M}+\mathrm{H}]^{+}, t_{\mathrm{R}}=1.52 \mathrm{~min}\right)$. These characterisation data are in accordance with that previously reported in the literature [43].

4'-Hydroxy-7-methoxyisoflavone (43). A mixture of phenylethanone 41 (2.00 g, $7.74 \mathrm{mmol}), \mathrm{BF}_{3} \cdot \mathrm{OEt}_{2}$ $(3.90 \mathrm{~mL}, 31.1 \mathrm{mmol})$ and $\mathrm{MeSO}_{2} \mathrm{Cl}(1.80 \mathrm{~mL}, 23.3 \mathrm{mmol})$ in dry DMF $(50 \mathrm{~mL})$ was reacted according to GP-H. The crude residue was purified by flash column chromatography $\left(\mathrm{SiO}_{2}, \mathrm{PE} / \mathrm{EtOAc} ; 2: 1\right)$ to afford isoflavone $43(1.18 \mathrm{~g}, 57 \%)$ as a white powdery solid. m.p. $232-234{ }^{\circ} \mathrm{C}$. TLC $R_{\mathrm{f}}=0.39$ (PE/EtOAc; 1:1). IR $\gamma_{\max }$ (neat) $/ \mathrm{cm}^{-1}: 3210 \mathrm{~m}(\mathrm{br})(\mathrm{O}-\mathrm{H}$ str), 3014w (C-H str), $1621 \mathrm{~s}(\mathrm{C}=\mathrm{O}$ str), $1583 \mathrm{~s}(\mathrm{C}=\mathrm{C}$ str), $1563 \mathrm{~m}\left(\mathrm{C}=\mathrm{C}\right.$ str), 1517s (C=C str), 1439s, 1373w, 1253s, 1205w, 1170w, 1095m, 1050m, 1019m. ${ }^{1} \mathrm{H}-\mathrm{NMR}$ $\left(500 \mathrm{MHz},\left(\mathrm{CD}_{3}\right)_{2} \mathrm{CO}\right): \delta 3.97\left(3 \mathrm{H}, \mathrm{s},-\mathrm{OCH}_{3}\right), 6.89(2 \mathrm{H}, \mathrm{d}, J=8.4 \mathrm{~Hz}, \mathrm{ArH}), 7.04-7.07(2 \mathrm{H}, \mathrm{m}, \mathrm{ArH}), 7.48$ $(2 \mathrm{H}, \mathrm{d}, J=8.8 \mathrm{~Hz}, \mathrm{ArH}), 8.10(1 \mathrm{H}, \mathrm{d}, J=8.8 \mathrm{~Hz}, \mathrm{ArH}), 8.19(1 \mathrm{H}, \mathrm{s},-\mathrm{C}=\mathrm{CH}), 8.44(1 \mathrm{H}, \mathrm{s}, \mathrm{OH}) .{ }^{13} \mathrm{C}-\mathrm{NMR}$ $\left(500 \mathrm{MHz},\left(\mathrm{CD}_{3}\right)_{2} \mathrm{CO}\right): \delta 55.8,100.4,114.7,115.2,118.5,123.7,124.7,127.4,130.4,152.7,157.6,158.2$, 164.4, 175.1. LCMS (ES+) $m / z=269.1\left([\mathrm{M}+\mathrm{H}]^{+}, t_{\mathrm{R}}=1.41 \mathrm{~min}\right)$. These characterisation data are in accordance with that previously reported in the literature [42]. 
7-Hydroxy-4'-methoxyisoflavone (44). A mixture of phenylethanone 42 (2.02 g, $7.82 \mathrm{mmol}), \mathrm{BF}_{3} \cdot \mathrm{OEt}_{2}$ $(3.90 \mathrm{~mL}, 31.1 \mathrm{mmol})$ and $\mathrm{MeSO}_{2} \mathrm{Cl}(1.80 \mathrm{~mL}, 23.2 \mathrm{mmol})$ in dry DMF $(50 \mathrm{~mL})$ was reacted according to GP-H. The crude residue was purified by flash column chromatography $\left(\mathrm{SiO}_{2}, \mathrm{PE} / \mathrm{EtOAc} ; 1: 1\right)$ and recrystallized from $\mathrm{CHCl}_{3}$ to afford isoflavone $44(1.62 \mathrm{~g}, 77 \%)$ as a dark yellow powdery solid. m.p. $242-244{ }^{\circ} \mathrm{C}$. TLC $R_{\mathrm{f}}=0.26$ (PE/EtOAc; 1:1). IR $v_{\max }$ (neat) $/ \mathrm{cm}^{-1}: 3126 \mathrm{~s}(\mathrm{br})(\mathrm{O}-\mathrm{H} \mathrm{str}), 2993 \mathrm{w}$ (C-H str), 2836w (C-H str), 1637m (C=O str), 1621m, 1594s (C=C str), 1568m (C=C str), 1512s (C=C str), 1451s, $1384 \mathrm{~m}, 1273 \mathrm{~m}, 1178 \mathrm{~s}, 1099 \mathrm{~m}, 1045 \mathrm{~m}, 1025 \mathrm{~s} .{ }^{1} \mathrm{H}-\mathrm{NMR}\left(500 \mathrm{MHz},\left(\mathrm{CD}_{3}\right)_{2} \mathrm{CO}\right): \delta 3.20(1 \mathrm{H}, \mathrm{br} \mathrm{s}, \mathrm{OH})$, $3.83\left(3 \mathrm{H}, \mathrm{s},-\mathrm{OCH}_{3}\right), 6.92(1 \mathrm{H}, \mathrm{d}, J=2.0 \mathrm{~Hz}, \mathrm{ArH}), 6.98(2 \mathrm{H}, \mathrm{d}, J=9.2 \mathrm{~Hz}, \mathrm{ArH}), 7.01(1 \mathrm{H}, \mathrm{dd}, J=8.8$, $2.4 \mathrm{~Hz}, \mathrm{ArH}), 7.56(2 \mathrm{H}, \mathrm{d}, J=8.8 \mathrm{~Hz}, \mathrm{ArH}), 8.07(1 \mathrm{H}, \mathrm{d}, J=8.4 \mathrm{~Hz}, \mathrm{ArH}), 8.18(1 \mathrm{H}, \mathrm{s},-\mathrm{C}=\mathrm{CH}) .{ }^{13} \mathrm{C}-\mathrm{NMR}$ $\left(500 \mathrm{MHz},\left(\mathrm{CD}_{3}\right)_{2} \mathrm{CO}\right): \delta 54.9,102.5,113.7,115.1,117.9,124.3,124.9,127.8,130.4,152.8,158.2,159.8$, 162.6, 175.0. LCMS (ES+) $m / z=269.1\left([\mathrm{M}+\mathrm{H}]^{+}, t_{\mathrm{R}}=1.47 \mathrm{~min}\right)$. These characterisation data are in accordance with that previously reported in the literature [44].

7-Methoxy-(4'-(prop-2-yn-1-yloxy)isoflavone (45). A mixture of isoflavone 43 (602 mg, $2.25 \mathrm{mmol}$ ), propargyl bromide $(0.50 \mathrm{~mL}, 5.61 \mathrm{mmol})$ and anhydrous $\mathrm{K}_{2} \mathrm{CO}_{3}(1.09 \mathrm{~g}, 7.91 \mathrm{mmol})$ in dry acetone $(50 \mathrm{~mL})$ was reacted according to GP-C. The crude residue was purified by flash column chromatography $\left(\mathrm{SiO}_{2}, 1 \% \mathrm{MeOH} / \mathrm{CH}_{2} \mathrm{Cl}_{2}\right)$ to afford isoflavone $45(641 \mathrm{mg}, 93 \%)$ as a white powdery solid. m.p. $162-164{ }^{\circ} \mathrm{C}$. TLC $R_{\mathrm{f}}=0.45\left(1 \% \mathrm{MeOH} / \mathrm{CH}_{2} \mathrm{Cl}_{2}\right)$. IR $v_{\max }$ (neat) $/ \mathrm{cm}^{-1}: 3289 \mathrm{~m}(\mathrm{C} \equiv \mathrm{C}-\mathrm{H}$ str), 3083w (C-H str), 2953w (C-H str), 1645s (C=O str), 1624s, 1606s, 1579m (C=C str), 1513s (C=C str), 1456m, 1442s, 1378s, 1285m, 1262s, 1245s, 1192s, 1176s, 1103s, 1050w, 1017s. ${ }^{1} \mathrm{H}-\mathrm{NMR}(500 \mathrm{MHz}$, $\left.\mathrm{CDCl}_{3}\right): \delta 2.54\left(1 \mathrm{H}, \mathrm{t}, J=2.4 \mathrm{~Hz},-\mathrm{OCH}_{2} \mathrm{C} \equiv \mathrm{CH}\right), 3.92\left(3 \mathrm{H}, \mathrm{s},-\mathrm{OCH}_{3}\right), 4.73(2 \mathrm{H}, \mathrm{d}, J=2.4 \mathrm{~Hz}$, $\left.-\mathrm{OCH}_{2} \mathrm{C} \equiv \mathrm{CH}\right), 6.86(2 \mathrm{H}, \mathrm{d}, J=2.0 \mathrm{~Hz}, \mathrm{ArH}), 7.00(1 \mathrm{H}, \mathrm{dd}, J=8.8,2.0 \mathrm{~Hz}, \mathrm{ArH}), 7.05(2 \mathrm{H}, \mathrm{d}, J=8.8 \mathrm{~Hz}$, $\mathrm{ArH}), 7.52(2 \mathrm{H}, \mathrm{d}, J=8.8 \mathrm{~Hz}, \mathrm{ArH}), 7.93(1 \mathrm{H}, \mathrm{s},-\mathrm{C}=\mathrm{CH}), 8.21(2 \mathrm{H}, \mathrm{d}, J=8.8 \mathrm{~Hz}, \mathrm{ArH}) .{ }^{13} \mathrm{C}-\mathrm{NMR}$ (500 MHz, $\mathrm{CDCl}_{3}$ ): $\delta 55.8,75.6,78.5,100.1,114.5,114.9,118.4,124.7,125.2,127.8,130.1,152.1,157.5$, 157.9, 164.0, 175.7. LCMS (ES+) $m / z=307.1\left([\mathrm{M}+\mathrm{H}]^{+}, t_{\mathrm{R}}=1.59 \mathrm{~min}\right)$. HRMS (ESI+) $m / z=307.0959$ $[\mathrm{M}+\mathrm{H}]^{+}$found, $\mathrm{C}_{19} \mathrm{H}_{15} \mathrm{O}_{4}{ }^{+}$required 307.0965.

4'-Methoxy-7-(prop-2-yn-1-yloxy)isoflavone (46). A mixture of isoflavone 44 (810 mg, $3.02 \mathrm{mmol})$, propargyl bromide $(0.55 \mathrm{~mL}, 6.17 \mathrm{mmol})$ and anhydrous $\mathrm{K}_{2} \mathrm{CO}_{3}(1.25 \mathrm{~g}, 9.01 \mathrm{mmol})$ in dry acetone $(50 \mathrm{~mL})$ was reacted according to GP-C. The crude residue was purified by flash column chromatography $\left(\mathrm{SiO}_{2}, \mathrm{CH}_{2} \mathrm{Cl}_{2}\right)$ to afford isoflavone $46(550 \mathrm{mg}, 60 \%)$ as a white powdery solid. m.p. $150-152{ }^{\circ} \mathrm{C}$. TLC $R_{\mathrm{f}}=0.32\left(0.5 \% \mathrm{MeOH} / \mathrm{CH}_{2} \mathrm{Cl}_{2}\right)$. IR $v_{\max }$ (neat) $/ \mathrm{cm}^{-1}: 3281 \mathrm{~m}(\mathrm{C} \equiv \mathrm{C}-\mathrm{H}$ str), $3259 \mathrm{~m}$, 3078w (C-H str), 2950w (C-H str), 2116w (C $\equiv \mathrm{C}$ str), 1624s (C=O str), 1608s, 1597s (C=C str), 1565m $(C=C$ str), 1513s $(C=C$ str), 1440s, 1373m, 1326w, 1295m, 1271s, 1237s, 1176s, 1109w, 1095s, 1052m, 1033s, 1016s. ${ }^{1} \mathrm{H}-\mathrm{NMR}\left(500 \mathrm{MHz}, \mathrm{CDCl}_{3}\right): \delta 2.61\left(1 \mathrm{H}, \mathrm{t}, J=2.0 \mathrm{~Hz},-\mathrm{OCH}_{2} \mathrm{C} \equiv \mathrm{CH}\right), 3.85\left(3 \mathrm{H}, \mathrm{s},-\mathrm{OCH}_{3}\right)$, $4.81\left(1 \mathrm{H}, \mathrm{d}, J=2.4 \mathrm{~Hz},-\mathrm{OCH}_{2} \mathrm{C} \equiv \mathrm{CH}\right), 6.98(2 \mathrm{H}, \mathrm{d}, J=8.4 \mathrm{~Hz}, \mathrm{ArH}), 6.98(1 \mathrm{H}, \mathrm{d}, J=2.4 \mathrm{~Hz}, \mathrm{ArH})$, $7.05(1 \mathrm{H}, \mathrm{dd}, J=8.8,2.4 \mathrm{~Hz}, \mathrm{ArH}), 7.51(2 \mathrm{H}, \mathrm{d}, J=8.8 \mathrm{~Hz}, \mathrm{ArH}), 7.93(1 \mathrm{H}, \mathrm{s},-\mathrm{C}=\mathrm{CH}), 8.24(1 \mathrm{H}, \mathrm{d}$, $J=8.8 \mathrm{~Hz}, \mathrm{ArH}) .{ }^{13} \mathrm{C}-\mathrm{NMR}\left(500 \mathrm{MHz}, \mathrm{CDCl}_{3}\right): \delta 55.3,56.2,76.6,77.3,101.5,113.9,114.8,119.0,124.1$, $124.9,127.9,130.1,152.1,157.6,159.6,161.6,175.8$. LCMS (ES+) $m / z=307.1\left([\mathrm{M}+\mathrm{H}]^{+}, t_{\mathrm{R}}=1.62 \mathrm{~min}\right)$. These characterisation data are in accordance with that previously reported in the literature [45].

4-(Prop-2-yn-1-yloxy)-2H-chromen-2-one (48). A mixture of 4-hydroxycoumarin 47 (5.06 g, $31.2 \mathrm{mmol}$ ), propargyl bromide $(6.20 \mathrm{~mL}, 69.6 \mathrm{mmol})$ and anhydrous $\mathrm{K}_{2} \mathrm{CO}_{3}(8.74 \mathrm{~g}, 63.2 \mathrm{mmol})$ in dry acetone $(100 \mathrm{~mL})$ was reacted according to GP-C. The crude residue was purified by flash column chromatography $\left(\mathrm{SiO}_{2}, \mathrm{CH}_{2} \mathrm{Cl}_{2}\right)$ to afford coumarin $48(3.60 \mathrm{~g}, 58 \%)$ as a white fluffy solid. m.p. $154-156^{\circ} \mathrm{C}$. TLC $R_{\mathrm{f}}=0.24\left(\mathrm{CH}_{2} \mathrm{Cl}_{2}\right)$. IR $v_{\max }$ (neat) $/ \mathrm{cm}^{-1}: 3281 \mathrm{~m}(\mathrm{C} \equiv \mathrm{C}-\mathrm{H}$ str), 3240m, 3078w $(\mathrm{C}-\mathrm{H}$ str), 2131w ( $\mathrm{C} \equiv \mathrm{C}$ str), 1714s (C=O str), 1688m, 1622s, 1610m (C=C str), 1568m (C=C str), 1493m, 1453m, 1409m, 1361m, 1329w, 1274s, 1248s, 1194w, 1179m, 1155w, 1145m, 1107s, $1032 \mathrm{w} .{ }^{1} \mathrm{H}-\mathrm{NMR}(500 \mathrm{MHz}$, $\left.\mathrm{CDCl}_{3}\right): \delta 2.68\left(1 \mathrm{H}, \mathrm{t}, J=2.4 \mathrm{~Hz},-\mathrm{OCH}_{2} \mathrm{C} \equiv \mathrm{CH}\right), 4.88\left(2 \mathrm{H}, \mathrm{d}, J=2.4 \mathrm{~Hz},-\mathrm{OCH}_{2} \mathrm{C} \equiv \mathrm{CH}\right), 5.84(1 \mathrm{H}, \mathrm{s}$, $-\mathrm{C}=\mathrm{CH}), 7.29-7.34(2 \mathrm{H}, \mathrm{m}, \mathrm{ArH}), 7.57(1 \mathrm{H}, \mathrm{t}, J=8.4 \mathrm{~Hz}, \mathrm{ArH}), 7.84(1 \mathrm{H}, \mathrm{dd}, J=8.0,1.2 \mathrm{~Hz}, \mathrm{ArH})$. ${ }^{13} \mathrm{C}-\mathrm{NMR}\left(500 \mathrm{MHz}, \mathrm{CDCl}_{3}\right): \delta 56.8,75.7,77.9,91.7,115.4,116.8,123.1,124.0,132.6,153.3,162.4,164.2$. 
LCMS (ES+) $m / z=201.1\left([\mathrm{M}+\mathrm{H}]^{+}, t_{\mathrm{R}}=1.47 \mathrm{~min}\right)$. These characterisation data are in accordance with that previously reported in the literature [46].

2-(2-Chloro-1-iminoethyl)-1,3,5-benzentriol hydrochloride (50). To a stirred solution of phloroglucinol 49 $(5.02 \mathrm{~g}, 39.8 \mathrm{mmol})$ and chloroacetonitrile $(2.50 \mathrm{~mL}, 39.5 \mathrm{mmol})$ in $\mathrm{Et}_{2} \mathrm{O}(100 \mathrm{~mL})$ was added anhydrous $\mathrm{ZnCl}_{2}(0.558 \mathrm{~g}, 4.09 \mathrm{mmol})$. The reaction mixture was cooled to $0{ }^{\circ} \mathrm{C}$ and $\mathrm{HCl}$ gas was bubbled through the solution for $30 \mathrm{~min}$. The resulting mixture was stirred at $0{ }^{\circ} \mathrm{C}$ for $3 \mathrm{~h}$ and further $24 \mathrm{~h}$ at room temperature. The resulting suspension was filtered and the precipitate was washed with ice-cold $\mathrm{Et}_{2} \mathrm{O}(2 \times 50 \mathrm{~mL})$ and suction-dried to afford hydrochloric salt $50(1.87 \mathrm{~g}, 20 \%)$ as a pale yellow-white powdery solid and was used without further purification in the next step. m.p. $240-242{ }^{\circ} \mathrm{C}$. IR $v_{\max }$ (neat) $/ \mathrm{cm}^{-1}: 3389 \mathrm{~m}(\mathrm{~N}-\mathrm{H}$ str), 3257m(br) (O-H str), 3184s(br) (O-H str), 2968w (C-H str), 1646m, 1616s (C=C str), 1591s ( $C=C$ str), 1531w (C=C str), 1457m, 1383m, 1366s, 1291m, 1248s, 1175s, 1128w, 1065m, 1050s, 1024w. ${ }^{1} \mathrm{H}-\mathrm{NMR}\left(500 \mathrm{MHz}, \mathrm{DMSO}-d_{6}\right): \delta 5.46\left(2 \mathrm{H}, \mathrm{s},-\mathrm{CH}_{2}-\right), 6.07(1 \mathrm{H}, \mathrm{d}, J=1.2 \mathrm{~Hz}, \mathrm{ArH}), 6.26$ $(1 \mathrm{H}, \mathrm{d}, J=1.2 \mathrm{~Hz}, \mathrm{ArH}), 9.90(1 \mathrm{H}, \mathrm{s}, \mathrm{OH}), 10.84(1 \mathrm{H}, \mathrm{s}, \mathrm{OH}), 11.80(1 \mathrm{H}, \mathrm{br} \mathrm{s}, \mathrm{NH}), 12.54(1 \mathrm{H}, \mathrm{s}, \mathrm{OH})$. ${ }^{13} \mathrm{C}-\mathrm{NMR}\left(500 \mathrm{MHz}\right.$, DMSO- $\left.d_{6}\right): \delta 75.3,90.1,96.9,99.3,160.4,172.8,173.7,176.0$. These characterisation data are in accordance with that previously reported in the literature [47].

2,4,6-Trihydroxy-2-chloroacetophenone (51). A mixture of imine salt $50(1.80 \mathrm{~g}, 7.56 \mathrm{mmol})$ and $1 \mathrm{M} \mathrm{HCl}$ $(100 \mathrm{~mL})$ were heated at reflux with stirring for $1 \mathrm{~h}$. The resulting red solution was blown under a steady stream of nitrogen and the residual solid was re-suspended in $\mathrm{H}_{2} \mathrm{O}(50 \mathrm{~mL})$. The precipitate was filtered, washed with ice-water $(2 \times 50 \mathrm{~mL})$, suction-dried and re-dissolved in EtOAc $(50 \mathrm{~mL})$. The organic solution was washed with $\mathrm{H}_{2} \mathrm{O}(2 \times 50 \mathrm{~mL})$, brine $(2 \times 50 \mathrm{~mL})$, dried over anhydrous $\mathrm{Na}_{2} \mathrm{SO}_{4}$, filtered and evaporated to dryness. The crude residue was purified by flash column chromatography $\left(\mathrm{SiO}_{2}, \mathrm{PE} / \mathrm{EtOAc} ; 1: 1\right)$ to afford acetophenone $51(389 \mathrm{mg}, 25 \%)$ as a white powdery solid. m.p. 236-238 ${ }^{\circ} \mathrm{C}$. TLC $R_{\mathrm{f}}=0.32$ (PE/EtOAc; 1:1). IR $v_{\max }$ (neat) $/ \mathrm{cm}^{-1}: 3422 \mathrm{~m}$ (br) $(\mathrm{O}-\mathrm{H} \mathrm{str}), 3372 \mathrm{~s}(\mathrm{br})$ (O-H str), 3068w (C-H str), 2962w (C-H str), 1640m (C=O str), 1598s (C=C str), 1521m (C=C str), 1456s, 1376s, 1331w, 1279m, 1213s, 1164s, 1073s, 1017m. ${ }^{1} \mathrm{H}-\mathrm{NMR}\left(500 \mathrm{MHz}, \mathrm{DMSO}-d_{6}\right): \delta 4.98(2 \mathrm{H}$, $\left.\mathrm{s},-\mathrm{COCH}_{2}-\right), 5.84(2 \mathrm{H}, \mathrm{s}, \mathrm{ArH}), 10.55(1 \mathrm{H}, \mathrm{s}, \mathrm{OH}), 12.07(2 \mathrm{H}, \mathrm{s}, \mathrm{OH}) .{ }^{13} \mathrm{C}-\mathrm{NMR}\left(500 \mathrm{MHz}, \mathrm{DMSO}-d_{6}\right)$ : $\delta 51.0,94.7,102.5,163.9,165.5,194.7$. LCMS (ES+) $m / z=203.0\left([\mathrm{M}+\mathrm{H}]^{+}, t_{\mathrm{R}}=1.26 \mathrm{~min}\right)$. These characterisation data are in accordance with that previously reported in the literature [26].

Dihydroxybenzofuran-3(2H)-one (52). To a stirred solution of acetophenone 51 (5.00 g, $24.7 \mathrm{mmol}$ ) in $\mathrm{MeOH}(100 \mathrm{~mL})$ was added $\mathrm{NaOMe}(4.88 \mathrm{~g}, 90.3 \mathrm{mmol})$ and the mixture was heated at reflux for $2 \mathrm{~h}$ under nitrogen. The reaction mixture was allowed to cool to room temperature, acidified with $1 \mathrm{M} \mathrm{HCl}$ and the solvent removed under reduced pressure. The resulting dark residue was then re-dissolved in EtOAc $(100 \mathrm{~mL})$. The organic solution was washed with $\mathrm{H}_{2} \mathrm{O}(2 \times 100 \mathrm{~mL})$, brine $(2 \times 100 \mathrm{~mL})$, dried over anhydrous $\mathrm{MgSO}_{4}$, filtered and evaporated to dryness. The crude residue was purified by flash column chromatography $\left(\mathrm{SiO}_{2}, \mathrm{PE} / \mathrm{EtOAc} ; 1: 1\right)$ to afford benzofuranone $52(2.90 \mathrm{~g}, 71 \%)$ as a pale brown-white powdery solid. m.p. $280-282{ }^{\circ} \mathrm{C}$. TLC $R_{\mathrm{f}}=0.16$ (PE/EtOAc; 1:1). IR $v_{\max }$ (neat) $/ \mathrm{cm}^{-1}$ : 3331s(br) (O-H str), 3164m(br) (O-H str), 3062w (C-H str), 1671m (C=O str), 1607s (C=C str), 1533w (C=C str), 1457m, 1422w, 1399m, 1369m, 1336m, 1261w, 1227m, 1157s, 1064s, 1042m, 1012m. ${ }^{1} \mathrm{H}-\mathrm{NMR}$ (500 MHz, DMSO- $\left.d_{6}\right): \delta 4.55$ (2H, s, $\left.-\mathrm{OCH}_{2} \mathrm{CO}-\right), 5.91(2 \mathrm{H}, \mathrm{s}, \mathrm{ArH}), 10.59$ (2H, br s, OH). ${ }^{13} \mathrm{C}-\mathrm{NMR}$ $\left(500 \mathrm{MHz}, \mathrm{DMSO}-d_{6}\right): \delta 74.9,90.1,96.2,102.7,157.5,167.6,175.6,194.0$. LCMS (ES+) $\mathrm{m} / z=167.1$ $\left([\mathrm{M}+\mathrm{H}]^{+}, t_{\mathrm{R}}=1.27 \mathrm{~min}\right)$. These characterisation data are in accordance with that previously reported in the literature [26].

4,6-Dimethoxybenzofuran-3(2H)-one (53). To a stirred solution of dihydroxybenzofuranone 52 (2.02 g, $12.2 \mathrm{mmol})$ in dry DMF $(50 \mathrm{~mL})$ were added $\mathrm{CH}_{3} \mathrm{I}(2.30 \mathrm{~mL}, 37.0 \mathrm{mmol})$ and anhydrous $\mathrm{K}_{2} \mathrm{CO}_{3}(3.35 \mathrm{~g}$, $24.2 \mathrm{mmol}$ ). The resulting dark red-brown suspension was heated at $80^{\circ} \mathrm{C}$ for $1 \mathrm{~h}$ under a nitrogen atmosphere. The reaction mixture was then allowed to cool to room temperature, poured into ice-water $(100 \mathrm{~mL})$ and extracted with EtOAc $(3 \times 100 \mathrm{~mL})$. The combined organic layer was washed with $\mathrm{H}_{2} \mathrm{O}$ $(3 \times 100 \mathrm{~mL})$, brine $(2 \times 100 \mathrm{~mL})$, dried over anhydrous $\mathrm{MgSO}_{4}$, filtered and evaporated to dryness. 
The crude residue was purified by flash column chromatography $\left(\mathrm{SiO}_{2}, \mathrm{PE} / \mathrm{EtOAc} ; 1: 1\right)$ to afford benzofuranone $53(1.87 \mathrm{~g}, 79 \%)$ as a pale yellow-white powdery solid. m.p. $148-150{ }^{\circ} \mathrm{C}$. TLC $R_{\mathrm{f}}=0.23$ (PE/EtOAc; 1:1). IR $v_{\max }$ (neat)/cm ${ }^{-1}:$ 2979w (C-H str), 2949w (C-H str), 1699s (C=O str), 1616s (C=C str), 1585s (C=C str), 1500m (C=C str), 1463m, 1431m, 1366m, 1342m, 1288m, 1217s, 1186s, 1160s, 1099s, 1052m, 1021m. ${ }^{1} \mathrm{H}-\mathrm{NMR}\left(500 \mathrm{MHz}_{\mathrm{CDCl}}\right): \delta 3.83\left(3 \mathrm{H}, \mathrm{s},-\mathrm{OCH}_{3}\right), 3.87\left(3 \mathrm{H}, \mathrm{s},-\mathrm{OCH}_{3}\right), 4.55$ $\left(2 \mathrm{H}, \mathrm{s},-\mathrm{OCH}_{2} \mathrm{CO}-\right), 5.97(1 \mathrm{H}, \mathrm{d}, J=1.6 \mathrm{~Hz}, \mathrm{ArH}), 6.11(1 \mathrm{H}, \mathrm{d}, J=2.0 \mathrm{~Hz}, \mathrm{ArH}) .{ }^{13} \mathrm{C}-\mathrm{NMR}(500 \mathrm{MHz}$, $\left.\mathrm{CDCl}_{3}\right): \delta 55.9,55.9,75.4,88.8,92.9,104.7,158.7,169.7,177.0,194.9$. LCMS (ES+) $m / z=195.1\left([\mathrm{M}+\mathrm{H}]^{+}\right.$, $\left.t_{\mathrm{R}}=1.52 \mathrm{~min}\right)$. These characterisation data are in accordance with that previously reported in the literature [26].

4,6-Dimethoxy-3'-hydroxyaurone (55). To a stirred solution of benzofuranone 53 (1.01 g, $5.20 \mathrm{mmol}) \mathrm{in}$ $\mathrm{MeOH}(20 \mathrm{~mL})$ was added 3-hydroxybenzaldehyde $54(0.760 \mathrm{~g}, 6.22 \mathrm{mmol})$ followed by the addition of $\mathrm{KOH}(1.50 \mathrm{~g}, 26.6 \mathrm{mmol})$ in $\mathrm{H}_{2} \mathrm{O}(20 \mathrm{~mL})$. The reaction mixture was stirred at room temperature for $2 \mathrm{~h}$ and then poured into $\mathrm{H}_{2} \mathrm{O}(2 \times 100 \mathrm{~mL})$. The resulting suspension was neutralized to $\mathrm{pH} 7$ with $3 \mathrm{M} \mathrm{HCl}$ and extracted with $\mathrm{CHCl}_{3}(3 \times 50 \mathrm{~mL})$. The combined organic layer was washed with $\mathrm{H}_{2} \mathrm{O}(3 \times 100 \mathrm{~mL})$, brine $(100 \mathrm{~mL})$, dried over anhydrous $\mathrm{MgSO}_{4}$, filtered and evaporated to dryness under reduced pressure. The crude residue was purified by flash column chromatography $\left(\mathrm{SiO}_{2}\right.$, $\mathrm{PE} / \mathrm{EtOAc} ; 1: 1)$ and recrystallized from $\mathrm{MeOH}$ to afford aurone $55(1.21 \mathrm{~g}, 78 \%)$ as a bright yellow powdery solid. m.p. $178-180{ }^{\circ} \mathrm{C}$. TLC $R_{\mathrm{f}}=0.35$ (PE/EtOAc; $\left.1: 2\right)$. IR $v_{\max }$ (neat) $/ \mathrm{cm}^{-1}: 3254 \mathrm{~m}(\mathrm{br})$ (O-H str), 2944w (C-H str), 2842w (C-H str), 1688m (C=O str), 1649m, 1612s (C=C str), 1586s (C=C str), $1501 \mathrm{~m}\left(\mathrm{C}=\mathrm{C}\right.$ str), 1447s, 1430w, 1338m, 1303m, 1249m, 1214s, 1153s, 1138m, 1087s, $1038 \mathrm{w} .{ }^{1} \mathrm{H}-\mathrm{NMR}$ $\left(500 \mathrm{MHz},\left(\mathrm{CD}_{3}\right)_{2} \mathrm{CO}\right): \delta 3.94\left(3 \mathrm{H}, \mathrm{s},-\mathrm{OCH}_{3}\right), 3.98\left(3 \mathrm{H}, \mathrm{s},-\mathrm{OCH}_{3}\right), 6.32(1 \mathrm{H}, \mathrm{d}, J=2.0 \mathrm{~Hz}, \mathrm{ArH}), 6.56(1 \mathrm{H}$, $\mathrm{d}, J=1.6 \mathrm{~Hz}, \mathrm{ArH}), 6.56(1 \mathrm{H}, \mathrm{s},-\mathrm{C}=\mathrm{CH}), 6.91(1 \mathrm{H}, \mathrm{ddd}, J=8.0,2.4,0.8 \mathrm{~Hz}, \mathrm{ArH}), 7.30(1 \mathrm{H}, \mathrm{t}, J=8.0 \mathrm{~Hz}$, $\mathrm{ArH}), 7.40(1 \mathrm{H}, \mathrm{d}, J=7.6 \mathrm{~Hz}, \mathrm{ArH}), 7.47(1 \mathrm{H}, \mathrm{t}, J=2.0 \mathrm{~Hz}, \mathrm{ArH}), 8.57(1 \mathrm{H}, \mathrm{s}, \mathrm{OH}) .{ }^{13} \mathrm{C}-\mathrm{NMR}(500 \mathrm{MHz}$, $\left.\left(\mathrm{CD}_{3}\right)_{2} \mathrm{CO}\right): \delta 55.9,56.2,89.7,94.4,104.8,109.4,116.8,117.5,122.8,130.0,134.1,148.1,157.9,159.7,169.2$, 169.5, 179.4. LCMS $(\mathrm{ES}+) \mathrm{m} / z=299.2\left([\mathrm{M}+\mathrm{H}]^{+}, t_{\mathrm{R}}=1.71 \mathrm{~min}\right)$. These characterisation data are in accordance with that previously reported in the literature [48].

4,6-Dimethoxy-3'-(prop-2-yn-1-yloxy)aurone (56). A mixture of aurone 55 (505 mg, $1.69 \mathrm{mmol})$, propargyl bromide $(0.30 \mathrm{~mL}, 3.37 \mathrm{mmol})$ and anhydrous $\mathrm{K}_{2} \mathrm{CO}_{3}(701 \mathrm{mg}, 5.07 \mathrm{mmol})$ in dry acetone $(30 \mathrm{~mL}) \mathrm{was}$ reacted according to GP-C. The crude residue was purified by flash column chromatography $\left(\mathrm{SiO}_{2}\right.$, $\left.0.5 \% \mathrm{MeOH} / \mathrm{CH}_{2} \mathrm{Cl}_{2}\right)$ to afford aurone $56(545 \mathrm{mg}, 96 \%)$ as a pale yellow-white powdery solid. m.p. $152-154{ }^{\circ} \mathrm{C}$. TLC $R_{\mathrm{f}}=0.46\left(1 \% \mathrm{MeOH} / \mathrm{CH}_{2} \mathrm{Cl}_{2}\right)$. IR $v_{\max }$ (neat) $/ \mathrm{cm}^{-1}: 3219 \mathrm{~m}(\mathrm{C} \equiv \mathrm{C}-\mathrm{H} \mathrm{str}), 3007 \mathrm{w}$ (C-H str), 2940w (C-H str), 2110w (C $\equiv$ C str), 1686s (C=O str), 1654m, 1612s (C=C str), 1589s (C=C str), 1504s (C=C str), 1454m, 1423m, 1347m, 1313m, 1266m, 1215s, 1154m, 1094s, 1040m, 1019w. ${ }^{1} \mathrm{H}-\mathrm{NMR}$ $\left(500 \mathrm{MHz} \mathrm{CDCl}_{3}\right): \delta 2.57\left(1 \mathrm{H}, \mathrm{t}, J=2.5 \mathrm{~Hz},-\mathrm{OCH}_{2} \mathrm{C} \equiv \mathrm{CH}\right), 3.91\left(3 \mathrm{H}, \mathrm{s},-\mathrm{OCH}_{3}\right), 3.95\left(3 \mathrm{H}, \mathrm{s},-\mathrm{OCH}_{3}\right)$, $4.75\left(2 \mathrm{H}, \mathrm{d}, J=2.5 \mathrm{~Hz},-\mathrm{OCH}_{2} \mathrm{C} \equiv \mathrm{CH}\right), 6.12(1 \mathrm{H}, \mathrm{d}, J=2.0 \mathrm{~Hz}, \mathrm{ArH}), 6.37(1 \mathrm{H}, \mathrm{d}, J=2.0 \mathrm{~Hz}, \mathrm{ArH})$, $6.72(1 \mathrm{H}, \mathrm{s},-\mathrm{C}=\mathrm{CH}), 6.99(1 \mathrm{H}, \mathrm{ddd}, J=8.0,2.5,1.0 \mathrm{~Hz}, \mathrm{ArH}), 7.35(1 \mathrm{H}, \mathrm{t}, J=8.0 \mathrm{~Hz}, \mathrm{ArH}), 7.46(1 \mathrm{H}$, $\mathrm{d}, J=7.5 \mathrm{~Hz}, \mathrm{ArH}), 7.54(1 \mathrm{H}, \mathrm{t}, J=1.5 \mathrm{~Hz}, \mathrm{ArH}) .{ }^{13} \mathrm{C}-\mathrm{NMR}\left(500 \mathrm{MHz}, \mathrm{CDCl}_{3}\right): \delta 55.9,56.1,56.2,75.7$, 78.3, 89.2, 94.0, 105.1, 110.3, 115.9, 117.1, 124.6, 129.7, 133.9, 148.0, 157.6, 159.4, 169.0, 180.6. LCMS $(\mathrm{ES}+) m / z=337.2\left([\mathrm{M}+\mathrm{H}]^{+}, t_{\mathrm{R}}=1.83 \mathrm{~min}\right)$. HRMS (ESI+) $m / z=337.1062[\mathrm{M}+\mathrm{H}]^{+}$found, $\mathrm{C}_{20} \mathrm{H}_{17} \mathrm{O}_{5}{ }^{+}$ required 337.1071 .

4-(2-Bromoethoxy)-2H-chromen-2-one (57). A mixture of 4-hydroxycoumarin 47 (5.14 g, $31.7 \mathrm{mmol})$, 1,2-dibromoethane $(3.19 \mathrm{~mL}, 37.0 \mathrm{mmol})$ and anhydrous $\mathrm{K}_{2} \mathrm{CO}_{3}(8.56 \mathrm{~g}, 61.9 \mathrm{mmol})$ in dry acetone $(100 \mathrm{~mL})$ was reacted according to GP-I. The crude residue was purified by flash column chromatography $\left(\mathrm{SiO}_{2}, \mathrm{CH}_{2} \mathrm{Cl}_{2}\right)$ to afford coumarin $57(1.33 \mathrm{~g}, 16 \%)$ as a white powdery solid. m.p. 176-178 ${ }^{\circ} \mathrm{C}$. TLC $R_{\mathrm{f}}=0.41$ (PE/EtOAc 1:1). IR $v_{\max }$ (neat) $/ \mathrm{cm}^{-1}: 3044 \mathrm{w}$ (C-H str), 2982w (C-H str), $1716 \mathrm{~s}(\mathrm{C}=\mathrm{O} \mathrm{str}), 1627 \mathrm{~s}, 1607 \mathrm{~s}, 1566 \mathrm{~m}(\mathrm{C}=\mathrm{C}$ str $), 1496 \mathrm{~m}, 1454 \mathrm{~m}, 1407 \mathrm{~s}, 1369 \mathrm{~s}, 1330 \mathrm{~m}, 1272 \mathrm{~m}, 1246 \mathrm{~s}$, 1225s, 1184s, 1147m, 1111s, 1067w, 1034s. ${ }^{1} \mathrm{H}-\mathrm{NMR}\left(500 \mathrm{MHz}, \mathrm{CDCl}_{3}\right): \delta 3.77(2 \mathrm{H}, \mathrm{t}, J=6.0 \mathrm{~Hz}$, $\left.-\mathrm{OCH}_{2} \mathrm{CH}_{2} \mathrm{Br}\right), 4.46\left(2 \mathrm{H}, \mathrm{t}, J=6.0 \mathrm{~Hz},-\mathrm{OCH}_{2} \mathrm{CH}_{2} \mathrm{Br}\right), 5.68(1 \mathrm{H}, \mathrm{s},-\mathrm{C}=\mathrm{CH}), 7.31(1 \mathrm{H}, \mathrm{t}, J=8.0 \mathrm{~Hz}, \mathrm{ArH})$, $7.34(1 \mathrm{H}, \mathrm{dd}, J=8.5,0.5 \mathrm{~Hz}, \mathrm{ArH}), 7.58(1 \mathrm{H}, \mathrm{t}, J=8.5 \mathrm{~Hz}, \mathrm{ArH}), 7.88(1 \mathrm{H}, \mathrm{dd}, J=7.5,1.5 \mathrm{~Hz}, \mathrm{ArH})$. 
${ }^{13} \mathrm{C}-\mathrm{NMR}\left(500 \mathrm{MHz}, \mathrm{CDCl}_{3}\right.$ ): $\delta 27.6,68.4,90.9,115.3,116.8,123.1,124.0,132.7,153.3,162.5,164.9$. LCMS $(\mathrm{ES}+) \mathrm{m} / z=271.0\left([\mathrm{M}+\mathrm{H}]^{+}, t_{\mathrm{R}}=1.53 \mathrm{~min}\right)$. These characterisation data are in accordance with that previously reported in the literature [49].

4-(2-Azidoethoxy)-2H-chromen-2-one (58). A mixture of coumarin 57 (679 mg, $2.52 \mathrm{mmol}$ ) and $\mathrm{NaN}_{3}$ (370 mg, $5.69 \mathrm{mmol})$ in dry DMF $(20 \mathrm{~mL})$ was reacted according to GP-J. The reaction mixture was worked up to afford coumarin $58(571 \mathrm{mg}, 98 \%)$ as an off-white powdery solid and was used without further purification. m.p. $150-152{ }^{\circ} \mathrm{C}$. TLC $R_{\mathrm{f}}=0.40\left(1 \% \mathrm{MeOH} / \mathrm{CH}_{2} \mathrm{Cl}_{2}\right) . \mathrm{IR} v_{\max }$ (neat)/ $\mathrm{cm}^{-1}$ : $3075 \mathrm{w}$ (C-H str), 2947w (C-H str), 2125s ( $\mathrm{N}_{3}$ str), 1732s (C=O str), 1623s, 1609s, 1566s (C=C str), 1495s, $1453 \mathrm{~m}, 1417 \mathrm{~s}, 1371 \mathrm{~s}, 1277 \mathrm{~m}, 1236 \mathrm{~s}, 1180 \mathrm{~s}, 1147 \mathrm{~s}, 1109 \mathrm{~s}, 1032 \mathrm{~s} .{ }^{1} \mathrm{H}-\mathrm{NMR}\left(500 \mathrm{MHz}, \mathrm{CDCl}_{3}\right): \delta 3.75(2 \mathrm{H}$, $\left.\mathrm{t}, J=5.0 \mathrm{~Hz},-\mathrm{OCH}_{2} \mathrm{CH}_{2} \mathrm{~N}_{3}\right), 4.32\left(2 \mathrm{H}, \mathrm{t}, J=5.0 \mathrm{~Hz},-\mathrm{OCH}_{2} \mathrm{CH}_{2} \mathrm{~N}_{3}\right), 5.70(1 \mathrm{H}, \mathrm{s},-\mathrm{C}=\mathrm{CH}), 7.31(1 \mathrm{H}, \mathrm{t}$, $J=8.0 \mathrm{~Hz}, \mathrm{ArH}), 7.34(1 \mathrm{H}, \mathrm{dd}, J=8.5,0.5 \mathrm{~Hz}, \mathrm{ArH}), 7.58(1 \mathrm{H}, \mathrm{t}, J=8.5 \mathrm{~Hz}, \mathrm{ArH}), 7.84(1 \mathrm{H}, \mathrm{dd}, J=8.0$, $1.5 \mathrm{~Hz}, \mathrm{ArH}) .{ }^{13} \mathrm{C}-\mathrm{NMR}\left(500 \mathrm{MHz}, \mathrm{CDCl}_{3}\right): \delta 49.6,68.2,90.9,115.2,116.8,123.0,124.1,132.7,153.3$, 162.5, 165.1. LCMS (ES+) $m / z=232.1\left([\mathrm{M}+\mathrm{H}]^{+}, t_{\mathrm{R}}=1.70 \mathrm{~min}\right)$. These characterisation data are in accordance with that previously reported in the literature [27].

4-(2-Bomoethoxy)benzaldehyde (59). A mixture of benzaldehyde 33 (20.0 g, $164 \mathrm{mmol}), 1$,2-dibromoethane $(28.5 \mathrm{~mL}, 331 \mathrm{mmol})$ and anhydrous $\mathrm{K}_{2} \mathrm{CO}_{3}(46.0 \mathrm{~g}$, $333 \mathrm{mmol})$ in dry acetone $(100 \mathrm{~mL})$ was reacted according to GP-I. The crude residue was purified by flash column chromatography $\left(\mathrm{SiO}_{2}, \mathrm{CH}_{2} \mathrm{Cl}_{2}\right)$ to afford benzaldehyde $59(13.2 \mathrm{~g}, 35 \%)$ as a white powdery solid. m.p. $56-58{ }^{\circ} \mathrm{C}$. TLC $R_{\mathrm{f}}=0.41$ $\left(\mathrm{CH}_{2} \mathrm{Cl}_{2}\right) . \mathrm{IR} v_{\max }$ (neat) $/ \mathrm{cm}^{-1}: 2967 \mathrm{w}$ (C-H str), 1679s (C=O str), 1601s (C=C str), 1577s (C=C str), $1508 \mathrm{~m}(\mathrm{C}=\mathrm{C}$ str) $, 1458 \mathrm{~m}, 1422 \mathrm{~m}, 1392 \mathrm{~m}, 1317 \mathrm{w}, 1300 \mathrm{~m}, 1282 \mathrm{~m}, 1249 \mathrm{~s}, 1229 \mathrm{~s}, 1211 \mathrm{~s}, 1160 \mathrm{~s}, 1107 \mathrm{w}, 1068 \mathrm{~s}$, 1008s. ${ }^{1} \mathrm{H}-\mathrm{NMR}\left(500 \mathrm{MHz}, \mathrm{CDCl}_{3}\right): \delta 3.68\left(2 \mathrm{H}, \mathrm{t}, J=6.4 \mathrm{~Hz},-\mathrm{OCH}_{2} \mathrm{CH}_{2} \mathrm{Br}\right), 4.39(2 \mathrm{H}, \mathrm{t}, J=6.4 \mathrm{~Hz}$, $\left.-\mathrm{OCH}_{2} \mathrm{CH}_{2} \mathrm{Br}\right), 7.03(2 \mathrm{H}, \mathrm{d}, J=8.8 \mathrm{~Hz}, \mathrm{ArH}), 7.86(2 \mathrm{H}, \mathrm{d}, J=8.8 \mathrm{~Hz}, \mathrm{ArH}), 9.91(1 \mathrm{H}, \mathrm{s}, \mathrm{CHO}) .{ }^{13} \mathrm{C}-\mathrm{NMR}$ $\left(500 \mathrm{MHz}, \mathrm{CDCl}_{3}\right): \delta 28.4,67.9,114.8,130.5,132.0,163.0,190.7$. LCMS (ES+) $\mathrm{m} / z=231.0\left([\mathrm{M}+\mathrm{H}]^{+}\right.$, $\left.t_{\mathrm{R}}=1.56 \mathrm{~min}\right)$. These characterisation data are in accordance with that previously reported in the literature [50].

4-(2-Azidoethoxy)benzaldehyde (60). A mixture of benzaldehyde 59 (12.8 g, $56.0 \mathrm{mmol})$ and $\mathrm{NaN}_{3}(7.36 \mathrm{~g}$, $113 \mathrm{mmol})$ in dry DMF (100 mL) was reacted according to GP-J. The reaction mixture was worked up to afford benzaldehyde $60(10.6 \mathrm{~g}, 99 \%)$ as a pale yellow viscous oil and was used without further purification. TLC $R_{\mathrm{f}}=0.38\left(\mathrm{CH}_{2} \mathrm{Cl}_{2}\right)$. IR $v_{\max }$ (neat) $/ \mathrm{cm}^{-1}: 2942 \mathrm{w}(\mathrm{C}-\mathrm{H} \mathrm{str}), 2837 \mathrm{w}(\mathrm{C}-\mathrm{H} \mathrm{str}), 2100 \mathrm{~s}$ $\left(\mathrm{N}_{3} \mathrm{str}\right), 1682 \mathrm{~s}(\mathrm{C}=\mathrm{O}$ str), 1598s (C=C str), 1578s (C=C str), 1508s (C=C str), 1427w, 1395w, 1304m, 1247s, 1213s, 1157s, 1110m, 1052m, 1008w. ${ }^{1} \mathrm{H}-\mathrm{NMR}\left(500 \mathrm{MHz}, \mathrm{CDCl}_{3}\right): \delta 3.64(2 \mathrm{H}, \mathrm{t}, J=5.0 \mathrm{~Hz}$, $\left.-\mathrm{OCH}_{2} \mathrm{CH}_{2} \mathrm{~N}_{3}\right), 4.22\left(2 \mathrm{H}, \mathrm{t}, J=5.0 \mathrm{~Hz},-\mathrm{OCH}_{2} \mathrm{CH}_{2} \mathrm{~N}_{3}\right), 7.02(2 \mathrm{H}, \mathrm{d}, J=8.5 \mathrm{~Hz}, \mathrm{ArH}), 7.84(2 \mathrm{H}, \mathrm{d}$, $J=9.0 \mathrm{~Hz}, \mathrm{ArH}), 9.88(1 \mathrm{H}, \mathrm{s}, \mathrm{CHO}) .{ }^{13} \mathrm{C}-\mathrm{NMR}\left(500 \mathrm{MHz}, \mathrm{CDCl}_{3}\right): \delta 49.9,67.1,114.7,130.3,131.9,163.0$, 190.7. LCMS (ES+) $m / z=193.2\left([\mathrm{M}+\mathrm{H}]^{+}, t_{\mathrm{R}}=1.62 \mathrm{~min}\right)$. These characterisation data are in accordance with that previously reported in the literature [50].

(E)-3-(4-(2-Azidoethoxy)phenyl)-1-(2-hydroxyphenyl)prop-2-en-1-one (61). A mixture of benzaldehyde 60 $(4.90 \mathrm{~g}, 25.6 \mathrm{mmol})$, acetophenone $10(3.09 \mathrm{~mL}, 25.7 \mathrm{mmol})$ and $\mathrm{KOH}(8.70 \mathrm{~g}, 155 \mathrm{mmol})$ in absolute $\mathrm{EtOH}(100 \mathrm{~mL})$ was reacted according to GP-D. The crude residue was purified by recrystallization from $\mathrm{MeOH}$ to afford chalcone $\mathbf{6 1}(6.13 \mathrm{~g}, 77 \%)$ as a bright yellow powdery solid. m.p. $142-144{ }^{\circ} \mathrm{C}$. TLC $R_{\mathrm{f}}=0.48$ (PE/EtOAc; 2:1). IR $v_{\max }$ (neat) $/ \mathrm{cm}^{-1}$ : 2932w (C-H str), 2874w (C-H str), 2107m (N $\mathrm{N}_{3} \mathrm{str}$ ), $2070 \mathrm{~m}, 1636 \mathrm{~s}$ ( $\mathrm{C}=\mathrm{O}$ str), 1602s, 1575s ( $\mathrm{C}=\mathrm{C}$ str), 1560s ( $\mathrm{C}=\mathrm{C}$ str), 1508s ( $\mathrm{C}=\mathrm{C}$ str), 1488s, $1424 \mathrm{~m}, 1345 \mathrm{~m}$, $1299 \mathrm{~m}, 1271 \mathrm{~m}, 1244 \mathrm{~m}, 1201 \mathrm{~m}, 1059 \mathrm{~m}, 1031 \mathrm{~m} .{ }^{1} \mathrm{H}-\mathrm{NMR}\left(500 \mathrm{MHz}, \mathrm{CDCl}_{3}\right): \delta 3.63(2 \mathrm{H}, \mathrm{t}, J=5.0 \mathrm{~Hz}$, $\left.-\mathrm{OCH}_{2} \mathrm{CH}_{2} \mathrm{~N}_{3}\right), 4.19\left(2 \mathrm{H}, \mathrm{t}, J=5.0 \mathrm{~Hz},-\mathrm{OCH}_{2} \mathrm{CH}_{2} \mathrm{~N}_{3}\right), 6.93-6.98(3 \mathrm{H}, \mathrm{m}, \mathrm{ArH}), 7.03(1 \mathrm{H}, \mathrm{dd}, J=8.5$, $1.0 \mathrm{~Hz}, \mathrm{ArH}), 7.49(1 \mathrm{H}, \mathrm{t}, J=8.5 \mathrm{~Hz}, \mathrm{ArH}), 7.54(1 \mathrm{H}, \mathrm{d}, J=15.5 \mathrm{~Hz},-\mathrm{CH}=\mathrm{CHCO}-), 7.63(2 \mathrm{H}, \mathrm{d}, J=8.5 \mathrm{~Hz}$, $\mathrm{ArH}), 7.89(1 \mathrm{H}, \mathrm{d}, J=15.5 \mathrm{~Hz},-\mathrm{CH}=\mathrm{CHCO}-), 7.92(1 \mathrm{H}, \mathrm{dd}, J=8.5,2.0 \mathrm{~Hz}, \mathrm{ArH}), 12.94(1 \mathrm{H}, \mathrm{s}, \mathrm{OH})$. ${ }^{13} \mathrm{C}-\mathrm{NMR}\left(500 \mathrm{MHz}, \mathrm{CDCl}_{3}\right): \delta 50.0,67.0,115.0,117.9,118.5,118.7,120.0,127.9,129.5,130.5,136.2$, 145.0, 160.5, 163.5, 193.5. LCMS (ES+) $m / z=310.1\left([\mathrm{M}+\mathrm{H}]^{+}, t_{\mathrm{R}}=1.80 \mathrm{~min}\right)$. These characterisation data are in accordance with that previously reported in the literature [51]. 
1-(4-(2-Bromoethoxy)-3-methoxyphenyl)ethan-1-one (62). A mixture of acetophenone 11 (10.0 g, 60.3 mmol), 1,2-dibromoethane $(10.5 \mathrm{~mL}, 122 \mathrm{mmol})$ and anhydrous $\mathrm{K}_{2} \mathrm{CO}_{3}(12.6,91.5 \mathrm{mmol})$ in dry DMF (100 mL) was reacted according to GP-I. The crude residue was purified by flash column chromatography $\left(\mathrm{SiO}_{2}\right.$, $\left.\mathrm{CH}_{2} \mathrm{Cl}_{2}\right)$ to afford acetophenone $62(3.90 \mathrm{~g}, 24 \%)$ as a white powdery solid. m.p. 98-100 ${ }^{\circ} \mathrm{C}$. TLC $R_{\mathrm{f}}=0.16\left(\mathrm{CH}_{2} \mathrm{Cl}_{2}\right)$. IR $v_{\max }$ (neat) $/ \mathrm{cm}^{-1}: 3075 \mathrm{w}$ (C-H str), 2971w (C-H str), 1760w, 1671s (C=O str), $1585 \mathrm{~s}(\mathrm{C}=\mathrm{C}$ str), 1507s (C=C str), 1460m, 1412s, 1385w, 1359m, 1263s, 1220s, 1171m, 1144s, 1076s, 1030s, 1008s. ${ }^{1} \mathrm{H}-\mathrm{NMR}\left(500 \mathrm{MHz}, \mathrm{CDCl}_{3}\right): \delta 2.58\left(3 \mathrm{H}, \mathrm{s},-\mathrm{COC}_{3}\right), 3.70\left(2 \mathrm{H}, \mathrm{t}, J=6.8 \mathrm{~Hz},-\mathrm{OCH}_{2} \mathrm{CH}_{2} \mathrm{Br}\right), 3.94$ $\left(3 \mathrm{H}, \mathrm{s},-\mathrm{OCH}_{3}\right), 4.41\left(2 \mathrm{H}, \mathrm{t}, J=6.8 \mathrm{~Hz},-\mathrm{OCH}_{2} \mathrm{CH}_{2} \mathrm{Br}\right), 6.91(1 \mathrm{H}, \mathrm{d}, J=8.8 \mathrm{~Hz}, \mathrm{ArH}), 7.55-7.57(2 \mathrm{H}, \mathrm{m}$, $\mathrm{ArH}) .{ }^{13} \mathrm{C}-\mathrm{NMR}\left(500 \mathrm{MHz}, \mathrm{CDCl}_{3}\right): \delta 26.3,28.2,56.1,68.7,111.0,112.2,123.0,131.4,149.5,151.7,196.7$. LCMS $(\mathrm{ES}+) m / z=275.0\left([\mathrm{M}+\mathrm{H}]^{+}, t_{\mathrm{R}}=1.43 \mathrm{~min}\right)$. These characterisation data are in accordance with that previously reported in the literature [52].

1-(4-(2-Azidoethoxy)-3-methoxyphenyl)ethan-1-one (63). A mixture of acetophenone 62 (3.50 g, $12.8 \mathrm{mmol})$ and $\mathrm{NaN}_{3}(1.25 \mathrm{~g}, 19.2 \mathrm{~mol})$ in DMF $(30 \mathrm{~mL})$ was reacted according to GP-J. The reaction mixture was worked up to afford phenylethanone $63(2.94 \mathrm{~g}, 98 \%)$ as a pale brown residual oil which solidified upon standing to give a pale brown crystalline solid and used without further purification. m.p. 58-60 ${ }^{\circ} \mathrm{C}$. TLC $R_{\mathrm{f}}=0.30\left(0.5 \% \mathrm{MeOH} / \mathrm{CH}_{2} \mathrm{Cl}_{2}\right)$. IR $v_{\max }$ (neat) $/ \mathrm{cm}^{-1}: 3088 \mathrm{w}(\mathrm{C}-\mathrm{H}$ str), $2956 \mathrm{w}(\mathrm{C}-\mathrm{H}$ str), 2110s ( $\left.\mathrm{N}_{3} \mathrm{str}\right), 2067 \mathrm{~m}, 1742 \mathrm{w}, 1671 \mathrm{~s}$ (C=O str), 1589s (C=C str), 1508s (C=C str), 1471m, 1419s, $1355 \mathrm{~m}, 1270 \mathrm{~s}, 1216 \mathrm{~s}, 1177 \mathrm{~m}, 1151 \mathrm{~s}, 1080 \mathrm{~m}, 1036 \mathrm{~s} .{ }^{1} \mathrm{H}-\mathrm{NMR}\left(500 \mathrm{MHz}, \mathrm{CDCl}_{3}\right): \delta 2.56\left(3 \mathrm{H}, \mathrm{s},-\mathrm{COCH}_{3}\right)$, $3.68\left(2 \mathrm{H}, \mathrm{t}, J=5.2 \mathrm{~Hz},-\mathrm{OCH}_{2} \mathrm{CH}_{2} \mathrm{~N}_{3}\right), 3.91\left(3 \mathrm{H}, \mathrm{s},-\mathrm{OCH}_{3}\right), 4.23\left(2 \mathrm{H}, \mathrm{t}, J=5.2 \mathrm{~Hz},-\mathrm{OCH}_{2} \mathrm{CH}_{2} \mathrm{~N}_{3}\right), 6.89$ $(1 \mathrm{H}, \mathrm{d}, J=8.0 \mathrm{~Hz}, \mathrm{ArH}), 7.53-7.55(2 \mathrm{H}, \mathrm{m}, \mathrm{ArH}) .{ }^{13} \mathrm{C}-\mathrm{NMR}\left(500 \mathrm{MHz}, \mathrm{CDCl}_{3}\right): \delta 26.2,50.0,56.0,67.7$, $110.7,111.9,122.9,131.2,149.4,151.9,196.7$. LCMS $(\mathrm{ES}+) \mathrm{m} / z=236.0\left([\mathrm{M}+\mathrm{H}]^{+}, t_{\mathrm{R}}=1.45 \mathrm{~min}\right)$. These characterisation data are in accordance with that previously reported in the literature [52].

(E)-1-(4-(2-Azidoethoxy)-3-methoxyphenyl)-3-(3,4-dimethoxyphenyl)prop-2-en-1-one (67). A mixture of benzaldehyde $64(1.48 \mathrm{~g}, 8.91 \mathrm{mmol})$, acetophenone $63(2.04 \mathrm{~g}, 8.67 \mathrm{mmol})$ and $\mathrm{KOH}(2.41 \mathrm{~g}, 43.0 \mathrm{mmol})$ in absolute EtOH (100 mL) was reacted according to GP-D. The crude residue was purified by flash column chromatography $\left(\mathrm{SiO}_{2}, \mathrm{PE} /\right.$ EtOAc; 5:1) and recrystallized from $\mathrm{MeOH}$ to afford chalcone $67(2.61 \mathrm{~g}, 79 \%)$ as a pale yellow-green powdery solid. m.p. $106-108^{\circ} \mathrm{C}$. TLC $R_{\mathrm{f}}=0.28(\mathrm{PE} / \mathrm{EtOAc}$; 1:1). IR $v_{\max }$ (neat) $/ \mathrm{cm}^{-1}: 2940 \mathrm{w}$ (C-H str), 2838w (C-H str), 2112s ( $\left.\mathrm{N}_{3} \mathrm{str}\right), 2068 \mathrm{~m}, 1648 \mathrm{~s}(\mathrm{C}=\mathrm{O}$ str), 1595s (C=C str), 1568s (C=C str), 1509s (C=C str), 1458m, 1419m, 1355w, 1312m, 1261s, 1242s, 1199m, 1159m, 1139s, 1036s, 1021s. ${ }^{1} \mathrm{H}-\mathrm{NMR}\left(500 \mathrm{MHz}, \mathrm{CDCl}_{3}\right): \delta 3.67\left(2 \mathrm{H}, \mathrm{t}, J=5.2 \mathrm{~Hz},-\mathrm{OCH}_{2} \mathrm{CH}_{2} \mathrm{~N}_{3}\right)$, $3.91\left(3 \mathrm{H}, \mathrm{s},-\mathrm{OCH}_{3}\right), 3.93\left(3 \mathrm{H}, \mathrm{s},-\mathrm{OCH}_{3}\right), 3.94\left(3 \mathrm{H}, \mathrm{s},-\mathrm{OCH}_{3}\right), 4.23\left(2 \mathrm{H}, \mathrm{t}, J=5.2 \mathrm{~Hz},-\mathrm{OCH}_{2} \mathrm{CH}_{2} \mathrm{~N}_{3}\right)$, $6.88(1 \mathrm{H}, \mathrm{d}, J=8.4 \mathrm{~Hz}, \mathrm{ArH}), 6.92(1 \mathrm{H}, \mathrm{d}, J=8.0 \mathrm{~Hz}, \mathrm{ArH}), 7.15(1 \mathrm{H}, \mathrm{d}, J=2.0 \mathrm{~Hz}, \mathrm{ArH}), 7.22(1 \mathrm{H}$, $\mathrm{dd}, J=8.4,2.0 \mathrm{~Hz}, \mathrm{ArH}), 7.39(1 \mathrm{H}, \mathrm{d}, J=15.6 \mathrm{~Hz},-\mathrm{CH}=\mathrm{CHCO}-), 7.61-7.65(2 \mathrm{H}, \mathrm{m}, \mathrm{ArH}), 7.75$ (1H, d, $J=15.2 \mathrm{~Hz},-\mathrm{CH}=\mathrm{CHCO}-) .{ }^{13} \mathrm{C}-\mathrm{NMR}\left(500 \mathrm{MHz}, \mathrm{CDCl}_{3}\right): \delta 49.9,55.8,56.0,67.7,110.1,111.0,111.4$, $111.9,119.4,122.4,122.8,127.8,132.2,144.2,149.1,149.6,151.2,151.7,188.5$. LCMS (ES+) $m / z=384.2$ $\left([\mathrm{M}+\mathrm{H}]^{+}, t_{\mathrm{R}}=3.98 \mathrm{~min}\right)$. These characterisation data are in accordance with that previously reported in the literature [52].

(E)-1-(4-(2-Azidoethoxy)-3-methoxyphenyl)-3-(2,3,4-trimethoxyphenyl)prop-2-en-1-one (68). A mixture of benzaldehyde $65(1.70 \mathrm{~g}, 8.66 \mathrm{mmol})$, acetophenone $63(2.02 \mathrm{~g}, 8.59 \mathrm{mmol})$ and $\mathrm{KOH}(2.39 \mathrm{~g}, 42.6 \mathrm{mmol})$ in absolute EtOH (100 mL) was reacted according to GP-D. The crude residue was purified by flash column chromatography $\left(\mathrm{SiO}_{2}, \mathrm{PE} / \mathrm{EtOAc} ; 5: 1\right)$ and recrystallized from $\mathrm{MeOH}$ to afford chalcone 68 $(2.61 \mathrm{~g}, 74 \%)$ as a pale yellow-green powdery solid. m.p. $114-116{ }^{\circ} \mathrm{C}$. TLC $R_{\mathrm{f}}=0.35(\mathrm{PE} / \mathrm{EtOAc} ; 1: 1)$. IR $v_{\max }$ (neat) $/ \mathrm{cm}^{-1}: 2945 \mathrm{w}$ (C-H str), 2836w (C-H str), 2119s (N str), 2072m, 1743w, 1644m (C=O str), 1588m (C=C str), 1560s ( $C=C$ str $), 1519 \mathrm{~m}(\mathrm{C}=\mathrm{C}$ str), 1494s, 1466m, 1420s, 1246s, 1149s, 1093s, $1042 \mathrm{~s}$. ${ }^{1} \mathrm{H}-\mathrm{NMR}\left(500 \mathrm{MHz}, \mathrm{CDCl}_{3}\right): \delta 3.66\left(2 \mathrm{H}, \mathrm{t}, J=5.2 \mathrm{~Hz},-\mathrm{OCH}_{2} \mathrm{CH}_{2} \mathrm{~N}_{3}\right), 3.87\left(3 \mathrm{H}, \mathrm{s},-\mathrm{OCH}_{3}\right), 3.88(3 \mathrm{H}, \mathrm{s}$, $\left.-\mathrm{OCH}_{3}\right), 3.92\left(3 \mathrm{H}, \mathrm{s},-\mathrm{OCH}_{3}\right), 3.93\left(3 \mathrm{H}, \mathrm{s},-\mathrm{OCH}_{3}\right), 4.23\left(2 \mathrm{H}, \mathrm{t}, J=5.0 \mathrm{~Hz},-\mathrm{OCH}_{2} \mathrm{CH}_{2} \mathrm{~N}_{3}\right), 6.70(1 \mathrm{H}, \mathrm{d}$, $J=8.8 \mathrm{~Hz}, \mathrm{ArH}), 6.91(1 \mathrm{H}, \mathrm{d}, J=8.8 \mathrm{~Hz}, \mathrm{ArH}), 7.37(1 \mathrm{H}, \mathrm{d}, J=8.8 \mathrm{~Hz}, \mathrm{ArH}), 7.55(1 \mathrm{H}, \mathrm{d}, J=16.0 \mathrm{~Hz}$, -CH=CHCO-), 7.61-7.63 (2H, m, ArH), 7.97 (1H, d, J = 15.6 Hz, -CH=CHCO-). ${ }^{13} \mathrm{C}-\mathrm{NMR}(500 \mathrm{MHz}$, $\left.\mathrm{CDCl}_{3}\right): \delta 49.9,55.9,60.8,61.2,67.7,107.5,111.5,112.0,120.8,121.9,122.4,123.7,132.4,139.3,142.3$, 
149.6, 151.6, 153.6, 155.6, 188.9. LCMS (ES+) $m / z=414.1\left([\mathrm{M}+\mathrm{H}]^{+}, t_{\mathrm{R}}=1.64 \mathrm{~min}\right)$. HRMS (ESI+) $m / z=414.1653[\mathrm{M}+\mathrm{H}]^{+}$found, $\mathrm{C}_{21} \mathrm{H}_{24} \mathrm{O}_{6} \mathrm{~N}_{3}{ }^{+}$required 414.1660.

(E)-1-(4-(2-Azidoethoxy)-3-methoxyphenyl)-3-(2,4,6-trimethoxyphenyl)prop-2-en-1-one (69). A mixture of benzaldehyde $66(1.69 \mathrm{~g}, 8.61 \mathrm{mmol})$, acetophenone $63(2.06 \mathrm{~g}, 8.76 \mathrm{mmol})$ and $\mathrm{KOH}(2.44 \mathrm{~g}, 43.5 \mathrm{mmol})$ in absolute $\mathrm{EtOH}(100 \mathrm{~mL})$ was reacted according to GP-D. The crude residue was purified by recrystallization from $\mathrm{MeOH}$ to afford chalcone $69(2.91 \mathrm{~g}, 82 \%)$ as a pale yellow-green powdery solid. m.p. $132-134{ }^{\circ} \mathrm{C}$. TLC $R_{\mathrm{f}}=0.29$ (PE/EtOAc; 1:1). IR $v_{\max }$ (neat) $/ \mathrm{cm}^{-1}: 3005 \mathrm{w}$ (C-H str), 2939w (C-H str), 2840w (C-H str), 2112m (N $\mathrm{N}_{3}$ str), 1647m (C=O str), 1596m (C=C str), 1567s (C=C str), 1518m (C=C str), 1493w, 1451m, 1417m, 1320s, 1266m, 1213s, 1147s, 1119s, 1026s. ${ }^{1} \mathrm{H}-\mathrm{NMR}\left(500 \mathrm{MHz}, \mathrm{CDCl}_{3}\right)$ : $\delta 3.70\left(2 \mathrm{H}, \mathrm{t}, J=5.2 \mathrm{~Hz},-\mathrm{OCH}_{2} \mathrm{CH}_{2} \mathrm{~N}_{3}\right), 3.87\left(3 \mathrm{H}, \mathrm{s},-\mathrm{OCH}_{3}\right), 3.92\left(6 \mathrm{H}, \mathrm{s}, 2 \times-\mathrm{OCH}_{3}\right), 3.96(3 \mathrm{H}, \mathrm{s}$, $\left.-\mathrm{OCH}_{3}\right), 4.27\left(2 \mathrm{H}, \mathrm{t}, J=5.2 \mathrm{~Hz},-\mathrm{OCH}_{2} \mathrm{CH}_{2} \mathrm{~N}_{3}\right), 6.16(2 \mathrm{H}, \mathrm{s}, \mathrm{ArH}), 6.95(1 \mathrm{H}, \mathrm{d}, J=8.4 \mathrm{~Hz}, \mathrm{ArH}), 7.62-7.65$ $(2 \mathrm{H}, \mathrm{m}, \mathrm{ArH}), 7.88(1 \mathrm{H}, \mathrm{d}, J=16.0 \mathrm{~Hz},-\mathrm{CH}=\mathrm{CHCO}-), 8.24(1 \mathrm{H}, \mathrm{d}, J=16.0 \mathrm{~Hz},-\mathrm{CH}=\mathrm{CHCO}-) .{ }^{13} \mathrm{C}-\mathrm{NMR}$ (500 MHz, $\mathrm{CDCl}_{3}$ ): $\delta$ 50.1, 55.4, 55.8, 56.1, 67.8, 90.5, 106.6, 111.8, 112.2, 121.8, 122.4, 133.3, 135.4, 149.6, 151.2, 161.6, 163.0, 190.5. LCMS (ES+) $m / z=414.1\left([\mathrm{M}+\mathrm{H}]^{+}, t_{\mathrm{R}}=1.67 \mathrm{~min}\right)$. HRMS (ESI+) $m / z=$ 414.1654 $[\mathrm{M}+\mathrm{H}]^{+}$found, $\mathrm{C}_{21} \mathrm{H}_{24} \mathrm{O}_{6} \mathrm{~N}_{3}{ }^{+}$required 414.1660.

(E)-1-(4-(2-Azidoethoxy)-3-methoxyphenyl)-3-(1-methyl-1H-pyrrol-2-yl)prop-2-en-1-one (70). A mixture of pyrrole aldehyde $7(1.40 \mathrm{~mL}, 13.0 \mathrm{mmol})$, acetophenone $63(3.03 \mathrm{~g}, 12.9 \mathrm{mmol})$ and $\mathrm{KOH}(4.33 \mathrm{~g}$, $77.2 \mathrm{mmol})$ in absolute EtOH $(100 \mathrm{~mL})$ was reacted according to GP-D. The crude product was purified by flash column chromatography $\left(\mathrm{SiO}_{2}, \mathrm{PE} / \mathrm{EtOAc} ; 5: 1\right)$ and recrystallized from $\mathrm{MeOH}$ to afford chalcone 70 as a bright yellow powdery solid $(1.56 \mathrm{~g}, 37 \%)$. m.p. $124-126^{\circ} \mathrm{C}$. TLC $R_{\mathrm{f}}=0.32$ (PE/EtOAc; 1:1). IR $v_{\max }$ (neat) $/ \mathrm{cm}^{-1}$ : 2946w (C-H str), 2107m ( $\mathrm{N}_{3}$ str), 2064w, 1739w, 1644m (C=O str), 1595s (C=C str), 1562s (C=C str), 1515m (C=C str), 1485m, 1342s, 1272s, 1238s, 1196s, 1174s, 1125s, 1059m, 1034s. ${ }^{1} \mathrm{H}-\mathrm{NMR}\left(500 \mathrm{MHz}, \mathrm{CDCl}_{3}\right): \delta 3.70\left(2 \mathrm{H}, \mathrm{t}, J=5.2 \mathrm{~Hz},-\mathrm{OCH}_{2} \mathrm{CH}_{2} \mathrm{~N}_{3}\right), 3.78\left(3 \mathrm{H}, \mathrm{s},-\mathrm{NCH}_{3}\right), 3.96(3 \mathrm{H}, \mathrm{s}$, $\left.-\mathrm{OCH}_{3}\right), 4.27\left(2 \mathrm{H}, \mathrm{t}, J=4.8 \mathrm{~Hz},-\mathrm{OCH}_{2} \mathrm{CH}_{2} \mathrm{~N}_{3}\right), 6.23-6.24(1 \mathrm{H}, \mathrm{m}, \mathrm{ArH}), 6.82(1 \mathrm{H}, \mathrm{t}, J=1.6 \mathrm{~Hz}, \mathrm{ArH})$, $6.85(1 \mathrm{H}, \mathrm{dd}, J=4.0,1.6 \mathrm{~Hz}, \mathrm{ArH}), 6.94(1 \mathrm{H}, \mathrm{d}, J=8.8 \mathrm{~Hz}, \mathrm{ArH}), 7.31(1 \mathrm{H}, \mathrm{d}, J=14.8 \mathrm{~Hz},-\mathrm{CH}=\mathrm{CHCO}-)$, 7.62-7.64 (2H, m, ArH), $7.81(1 \mathrm{H}, \mathrm{d}, J=15.2 \mathrm{~Hz},-\mathrm{CH}=\mathrm{CHCO}-) .{ }^{13} \mathrm{C}-\mathrm{NMR}\left(500 \mathrm{MHz}, \mathrm{CDCl}_{3}\right): \delta 34.4$, 50.1, 56.1, 67.8, 109.7, 111.5, 112.1, 112.1, 116.4, 122.2, 127.6, 130.3, 131.7, 132.7, 149.7, 151.6, 188.1. LCMS (ES+) $m / z=327.2\left([\mathrm{M}+\mathrm{H}]^{+}, t_{\mathrm{R}}=1.82 \mathrm{~min}\right)$. HRMS (ESI+) $m / z=327.1447[\mathrm{M}+\mathrm{H}]^{+}$found, $\mathrm{C}_{17} \mathrm{H}_{19} \mathrm{O}_{3} \mathrm{~N}_{4}{ }^{+}$required 327.1452.

4-(2-Bromoethoxy)-3-methoxy benzaldehyde (72). A mixture of benzaldehyde 71 (10.0 g, $65.9 \mathrm{mmol})$, 1,2-dibromoethane $(7.40 \mathrm{~mL}, 85.9 \mathrm{mmol})$ and anhydrous $\mathrm{K}_{2} \mathrm{CO}_{3}(11.8 \mathrm{~g}, 85.5 \mathrm{mmol})$ in dry acetone $(100 \mathrm{~mL})$ was reacted according to GP-I. The crude residue was purified by flash column chromatography $\left(\mathrm{SiO}_{2}, \mathrm{CH}_{2} \mathrm{Cl}_{2}\right)$ to afford benzaldehyde $72(4.24 \mathrm{~g}, 25 \%)$ as a white crystalline solid. m.p. $84-86{ }^{\circ} \mathrm{C}$. TLC $R_{\mathrm{f}}=0.37\left(\mathrm{CH}_{2} \mathrm{Cl}_{2}\right)$. IR $v_{\max }$ (neat) $/ \mathrm{cm}^{-1}: 2967 \mathrm{w}(\mathrm{C}-\mathrm{H} \mathrm{str}), 2849 \mathrm{w}$ (C-H str), $1697 \mathrm{~m}$ (C=O str), 1682s, 1672s, 1585s (C=C str), 1508s (C=C str), 1459m, 1444m, 1427m, 1397m, 1349w, $1279 \mathrm{~m}, 1266 \mathrm{~s}, 1233 \mathrm{~s}, 1215 \mathrm{w}, 1159 \mathrm{w}, 1134 \mathrm{~s}, 1015 \mathrm{~s}, 1003 \mathrm{~m} .{ }^{1} \mathrm{H}-\mathrm{NMR}\left(500 \mathrm{MHz}, \mathrm{CDCl}_{3}\right): \delta 3.70(2 \mathrm{H}$, $\left.\mathrm{t}, J=6.5 \mathrm{~Hz},-\mathrm{OCH}_{2} \mathrm{CH}_{2} \mathrm{Br}\right), 3.94\left(3 \mathrm{H}, \mathrm{s},-\mathrm{OCH}_{3}\right), 4.42\left(2 \mathrm{H}, \mathrm{t}, J=6.5 \mathrm{~Hz},-\mathrm{OCH}_{2} \mathrm{CH}_{2} \mathrm{Br}\right), 6.99(1 \mathrm{H}, \mathrm{d}$, $J=8.0 \mathrm{~Hz}, \mathrm{ArH}), 7.43-7.46(2 \mathrm{H}, \mathrm{m}, \mathrm{ArH}), 9.87(1 \mathrm{H}, \mathrm{s}, \mathrm{CHO}) .{ }^{13} \mathrm{C}-\mathrm{NMR}\left(500 \mathrm{MHz}, \mathrm{CDCl}_{3}\right): \delta 28.1,56.1$, $68.7,109.8,112.4,126.4,130.8,150.0,152.9,190.8$. LCMS (ES+) $m / z=261.0\left([\mathrm{M}+\mathrm{H}]^{+}, t_{\mathrm{R}}=1.49 \mathrm{~min}\right)$. These characterisation data are in accordance with that previously reported in the literature [53].

3-(2-Bromoethoxy)-4-methoxy benzaldehyde (73). A mixture of isovanillin 1 (10.3 g, $68.0 \mathrm{mmol})$, 1,2-dibromoethane $(7.36 \mathrm{~mL}, 85.4 \mathrm{mmol})$ and anhydrous $\mathrm{K}_{2} \mathrm{CO}_{3}(11.8 \mathrm{~g}, 85.7 \mathrm{mmol})$ in dry acetone $(100 \mathrm{~mL})$ was reacted according to GP-I. The crude residue was purified by flash column chromatography $\left(\mathrm{SiO}_{2}, \mathrm{CH}_{2} \mathrm{Cl}_{2}\right)$ to afford benzaldehyde $73(3.81 \mathrm{~g}, 22 \%)$ as a white powdery solid. m.p. 88-90 ${ }^{\circ} \mathrm{C}$. TLC $R_{\mathrm{f}}=0.38\left(\mathrm{CH}_{2} \mathrm{Cl}_{2}\right)$. IR $v_{\max }$ (neat) $/ \mathrm{cm}^{-1}: 2978 \mathrm{w}(\mathrm{C}-\mathrm{H} \mathrm{str}), 2840 \mathrm{w}$ (C-H str), $1677 \mathrm{~s}$ (C=O str), 1595m ( $\mathrm{C}=\mathrm{C}$ str), 1582s (C=C str), 1509s (C=C str), 1462w, 1434s, 1392m, 1259s, 1238s, 1162s, 1131s, 1072w, 1013s. ${ }^{1} \mathrm{H}-\mathrm{NMR}\left(500 \mathrm{MHz}, \mathrm{CDCl}_{3}\right): \delta 3.69\left(2 \mathrm{H}, \mathrm{t}, J=6.4 \mathrm{~Hz},-\mathrm{OCH}_{2} \mathrm{CH}_{2} \mathrm{Br}\right), 3.96(3 \mathrm{H}, \mathrm{s}$, $\left.-\mathrm{OCH}_{3}\right), 4.39\left(2 \mathrm{H}, \mathrm{t}, J=6.4 \mathrm{~Hz},-\mathrm{OCH}_{2} \mathrm{CH}_{2} \mathrm{Br}\right), 7.00(1 \mathrm{H}, \mathrm{d}, J=8.0 \mathrm{~Hz}, \mathrm{ArH}), 7.41(1 \mathrm{H}, \mathrm{d}, J=1.6 \mathrm{~Hz}$, 
$\mathrm{ArH}), 7.50(1 \mathrm{H}, \mathrm{dd}, J=8.0,1.6 \mathrm{~Hz}, \mathrm{ArH}), 9.84(1 \mathrm{H}, \mathrm{s}, \mathrm{CHO}) .{ }^{13} \mathrm{C}-\mathrm{NMR}\left(500 \mathrm{MHz}, \mathrm{CDCl}_{3}\right): \delta 28.5,56.2$, $68.8,111.1,111.6,127.4,130.0,148.0,155.0,190.6$. LCMS (ES+) $m / z=261.0\left([\mathrm{M}+\mathrm{H}]^{+}, t_{\mathrm{R}}=1.47 \mathrm{~min}\right)$. These characterisation data are in accordance with that previously reported in the literature [53].

4-(2-Azidoethoxy)-3-methoxybenzaldehyde (74). A mixture of benzaldehyde 72 (3.40 g, $13.1 \mathrm{mmol})$ and $\mathrm{NaN}_{3}(1.74 \mathrm{~g}, 26.8 \mathrm{mmol})$ in dry DMF $(20 \mathrm{~mL})$ was reacted according to GP-J. The reaction mixture was worked up to afford benzaldehyde $74(2.84 \mathrm{~g}, 98 \%)$ as a pale yellow viscous oil and was used without further purification. TLC $R_{\mathrm{f}}=0.21\left(\mathrm{CH}_{2} \mathrm{Cl}_{2}\right)$. IR $v_{\max }$ (neat) $/ \mathrm{cm}^{-1}: 2938 \mathrm{w}(\mathrm{C}-\mathrm{H} \mathrm{str}), 2831 \mathrm{w}$ (C-H str), 2106s ( $\left.\mathrm{N}_{3} \mathrm{str}\right), 1679 \mathrm{~s}$ (C=O str), 1586s (C=C str), 1507s (C=C str), 1457m, 1424m, 1396w, 1339w, 1263s, 1236m, 1194w, 1158w, 1134s, 1051w, 1030m. ${ }^{1} \mathrm{H}-\mathrm{NMR}\left(500 \mathrm{MHz}, \mathrm{CDCl}_{3}\right): \delta 3.66(2 \mathrm{H}$, $\left.\mathrm{t}, J=5.2 \mathrm{~Hz},-\mathrm{OCH}_{2} \mathrm{CH}_{2} \mathrm{~N}_{3}\right), 3.88\left(3 \mathrm{H}, \mathrm{s},-\mathrm{OCH}_{3}\right), 4.22\left(2 \mathrm{H}, \mathrm{t}, J=5.2 \mathrm{~Hz},-\mathrm{OCH}_{2} \mathrm{CH}_{2} \mathrm{~N}_{3}\right), 6.95(1 \mathrm{H}, \mathrm{d}$, $J=8.0 \mathrm{~Hz}, \mathrm{ArH}), 7.38-7.41(2 \mathrm{H}, \mathrm{m}, \mathrm{ArH}), 9.81(1 \mathrm{H}, \mathrm{s}, \mathrm{CHO}) .{ }^{13} \mathrm{C}-\mathrm{NMR}\left(500 \mathrm{MHz}, \mathrm{CDCl}_{3}\right): \delta 49.8,55.8$, 67.7, 109.5, 112.0, 126.1, 130.6, 149.9, 153.0, 190.7. LCMS (ES+) $\mathrm{m} / z=222.0\left([\mathrm{M}+\mathrm{H}]^{+}, t_{\mathrm{R}}=1.66 \mathrm{~min}\right)$. HRMS (ESI+) $m / z=222.0863[\mathrm{M}+\mathrm{H}]^{+}$found, $\mathrm{C}_{10} \mathrm{H}_{12} \mathrm{O}_{3} \mathrm{~N}_{3}{ }^{+}$required 222.0834.

3-(2-Azidoethoxy)-4-methoxybenzaldehyde (75). A mixture of benzaldehyde 73 (3.63 g, $14.0 \mathrm{mmol}$ ) and $\mathrm{NaN}_{3}(1.80 \mathrm{~g}, 27.7 \mathrm{mmol})$ in dry DMF $(20 \mathrm{~mL})$ was reacted according to GP-J. The reaction mixture was worked up to afford benzaldehyde $75(3.03 \mathrm{~g}, 98 \%)$ as a pale yellow viscous oil and was used without further purification. TLC $R_{\mathrm{f}}=0.26\left(\mathrm{CH}_{2} \mathrm{Cl}_{2}\right)$. IR $v_{\max }$ (neat) $/ \mathrm{cm}^{-1}: 2940 \mathrm{w}(\mathrm{C}-\mathrm{H} \mathrm{str}), 2838 \mathrm{w}$ (C-H str), 2094s ( $\left.\mathrm{N}_{3} \mathrm{str}\right), 1682 \mathrm{~s}(\mathrm{C}=\mathrm{O}$ str), 1596s (C=C str), 1583s (C=C str), 1508s (C=C str), 1435s, 1397w, 1339w, 1261s, 1236s, 1161m, 1134s, 1125s, 1051w, 1019s. ${ }^{1} \mathrm{H}-\mathrm{NMR}\left(500 \mathrm{MHz}, \mathrm{CDCl}_{3}\right): \delta 3.68$ $\left(2 \mathrm{H}, \mathrm{t}, J=5.2 \mathrm{~Hz},-\mathrm{OCH}_{2} \mathrm{CH}_{2} \mathrm{~N}_{3}\right), 3.96\left(3 \mathrm{H}, \mathrm{s},-\mathrm{OCH}_{3}\right), 4.25\left(2 \mathrm{H}, \mathrm{t}, J=5.2 \mathrm{~Hz},-\mathrm{OCH}_{2} \mathrm{CH}_{2} \mathrm{~N}_{3}\right), 7.00$ $(1 \mathrm{H}, \mathrm{d}, J=8.0 \mathrm{~Hz}, \mathrm{ArH}), 7.42(1 \mathrm{H}, \mathrm{d}, J=2.0 \mathrm{~Hz}, \mathrm{ArH}), 7.50(1 \mathrm{H}, \mathrm{dd}, J=8.4,2.0 \mathrm{~Hz}, \mathrm{ArH}), 9.85(1 \mathrm{H}, \mathrm{s}$, CHO). ${ }^{13} \mathrm{C}-\mathrm{NMR}\left(500 \mathrm{MHz}, \mathrm{CDCl}_{3}\right): \delta 50.0,56.1,67.9,110.9,111.1,127.5,129.9,148.3,155.1,190.7$. LCMS (ES+) $m / z=222.3\left([\mathrm{M}+\mathrm{H}]^{+}, t_{\mathrm{R}}=1.69 \mathrm{~min}\right)$. HRMS (ESI+) $m / z=244.0683[\mathrm{M}+\mathrm{Na}]^{+}$found, $\mathrm{C}_{10} \mathrm{H}_{11} \mathrm{O}_{3} \mathrm{~N}_{3} \mathrm{Na}^{+}$required 244.0693 .

(E)-3-(4-(2-Azidoethoxy)phenyl)-1-(2-hydroxy-4-methoxyphenyl)prop-2-en-1-one (76). A mixture of benzaldehyde 60 (5.00 g, $26.2 \mathrm{mmol})$, acetophenone $3(4.39 \mathrm{~g}, 26.4 \mathrm{mmol})$ and $\mathrm{KOH}(9.42 \mathrm{~g}, 168 \mathrm{mmol})$ in absolute EtOH $(100 \mathrm{~mL})$ was reacted according to GP-D. The crude residue was purified by recrystallization from $\mathrm{MeOH}$ to afford chalcone 76 (5.72 g, 64\%) as a bright yellow-orange powdery solid. m.p. $124-126{ }^{\circ} \mathrm{C}$. TLC $R_{\mathrm{f}}=0.36$ (PE/EtOAc; 2:1). IR $\gamma_{\max }$ (neat) $/ \mathrm{cm}^{-1}: 2940 \mathrm{w}$ (C-H str), 2873w (C-H str), 2109m ( $\left.\mathrm{N}_{3} \mathrm{str}\right), 2073 \mathrm{~m}, 1634 \mathrm{~m}$ (C=O str), 1604m, 1573s (C=C str), 1513s (C=C str), $1445 \mathrm{~m}, 1426 \mathrm{~m}, 1361 \mathrm{~s}, 1328 \mathrm{~m}, 1307 \mathrm{w}, 1279 \mathrm{~s}, 1250 \mathrm{w}, 1209 \mathrm{~s}, 1184 \mathrm{~s}, 1119 \mathrm{~s}, 1057 \mathrm{~m}, 1015 \mathrm{~s} .{ }^{1} \mathrm{H}-\mathrm{NMR}$ $\left(500 \mathrm{MHz}, \mathrm{CDCl}_{3}\right): \delta 3.65\left(2 \mathrm{H}, \mathrm{t}, J=5.2 \mathrm{~Hz},-\mathrm{OCH}_{2} \mathrm{CH}_{2} \mathrm{~N}_{3}\right), 3.87\left(3 \mathrm{H}, \mathrm{s},-\mathrm{OCH}_{3}\right), 4.22(2 \mathrm{H}, \mathrm{t}, J=5.2 \mathrm{~Hz}$, $\left.-\mathrm{OCH}_{2} \mathrm{CH}_{2} \mathrm{~N}_{3}\right), 6.49-6.51(2 \mathrm{H}, \mathrm{m}, \mathrm{ArH}), 6.98(2 \mathrm{H}, \mathrm{d}, J=8.8 \mathrm{~Hz}, \mathrm{ArH}), 7.48(1 \mathrm{H}, \mathrm{d}, J=15.2 \mathrm{~Hz}$, - $\mathrm{CH}=\mathrm{CHCO}-), 7.63(2 \mathrm{H}, \mathrm{d}, J=8.8 \mathrm{~Hz}, \mathrm{ArH}), 7.84(1 \mathrm{H}, \mathrm{d}, J=9.2 \mathrm{~Hz}, \mathrm{ArH}), 7.87(1 \mathrm{H}, \mathrm{d}, J=15.6 \mathrm{~Hz}$, -CH=CHCO-), $13.52(1 \mathrm{H}, \mathrm{s}, \mathrm{OH}) .{ }^{13} \mathrm{C}-\mathrm{NMR}\left(500 \mathrm{MHz}, \mathrm{CDCl}_{3}\right): \delta 50.6,55.6,67.1,101.0,107.7,114.1$, 115.0, 118.3, 128.2, 130.4, 131.1, 144.0, 160.3, 166.1, 166.6, 191.8. LCMS (ES+) $m / z=340.2\left([\mathrm{M}+\mathrm{H}]^{+}\right.$, $t_{\mathrm{R}}=1.74 \mathrm{~min}$ ). HRMS (ESI+) $m / z=340.1295[\mathrm{M}+\mathrm{H}]^{+}$found, $\mathrm{C}_{18} \mathrm{H}_{18} \mathrm{~N}_{3} \mathrm{O}_{4}{ }^{+}$required 340.1297.

(E)-3-(4-(2-Azidoethoxy)-3-methoxyphenyl)-1-(2-hydroxyphenyl)prop-2-en-1-one (77). A mixture of benzaldehyde $74(2.70 \mathrm{~g}, 12.2 \mathrm{mmol})$, acetophenone $10(1.50 \mathrm{~mL}, 12.5 \mathrm{mmol})$ and $\mathrm{KOH}(4.30 \mathrm{~g}$, $76.6 \mathrm{mmol})$ in absolute EtOH $(100 \mathrm{~mL})$ was reacted according to GP-D. The crude residue was purified by flash column chromatography $\left(\mathrm{SiO}_{2}, \mathrm{PE} / \mathrm{EtOAc} ; 5: 1\right)$ to afford chalcone $77(1.91 \mathrm{~g}, 46 \%)$ as a bright yellow-orange powdery solid. m.p. $84-86^{\circ} \mathrm{C}$. TLC $R_{\mathrm{f}}=0.25$ (PE/EtOAc; 3:1). IR $v_{\max }$ (neat) $/ \mathrm{cm}^{-1}$ : 2935w (C-H str), 2876w (C-H str), 2106s ( $\mathrm{N}_{3}$ str), 1635s (C=O str), 1580m (C=C str), 1562s (C=C str), 1508s (C=C str), 1489s, 1462w, 1441w, 1421w, 1372w, 1313w, 1255s, 1201s, 1168w, 1138s, 1057w, 1028s. ${ }^{1} \mathrm{H}-\mathrm{NMR}\left(500 \mathrm{MHz}, \mathrm{CDCl}_{3}\right): \delta 3.68\left(2 \mathrm{H}, \mathrm{t}, J=5.5 \mathrm{~Hz},-\mathrm{OCH}_{2} \mathrm{CH}_{2} \mathrm{~N}_{3}\right), 3.95\left(3 \mathrm{H}, \mathrm{s},-\mathrm{OCH}_{3}\right), 4.24(2 \mathrm{H}$, $\left.\mathrm{t}, J=5.5 \mathrm{~Hz},-\mathrm{OCH}_{2} \mathrm{CH}_{2} \mathrm{~N}_{3}\right), 6.92(1 \mathrm{H}, \mathrm{d}, J=8.0 \mathrm{~Hz}, \mathrm{ArH}), 6.93-6.96(1 \mathrm{H}, \mathrm{m}, \mathrm{ArH}), 7.03(1 \mathrm{H}, \mathrm{dd}$, $J=8.5,1.5 \mathrm{~Hz}, \mathrm{ArH}), 7.19(1 \mathrm{H}, \mathrm{d}, J=2.0 \mathrm{~Hz}, \mathrm{ArH}), 7.25(1 \mathrm{H}, \mathrm{dd}, J=8.5,1.5 \mathrm{~Hz}, \mathrm{ArH}), 7.50(1 \mathrm{H}, \mathrm{t}$, $J=8.5 \mathrm{~Hz}, \mathrm{ArH}), 7.53(1 \mathrm{H}, \mathrm{d}, J=15.5 \mathrm{~Hz},-\mathrm{CH}=\mathrm{CHCO}-), 7.87(1 \mathrm{H}, \mathrm{d}, J=15.5 \mathrm{~Hz},-\mathrm{CH}=\mathrm{CHCO}-), 7.93$ 
$(1 \mathrm{H}, \mathrm{dd}, J=8.0,1.5 \mathrm{~Hz}, \mathrm{ArH}), 12.91(1 \mathrm{H}, \mathrm{s}, \mathrm{OH}) .{ }^{13} \mathrm{C}-\mathrm{NMR}\left(500 \mathrm{MHz}, \mathrm{CDCl}_{3}\right): \delta 50.1,56.1,67.9,111.2$, $113.4,118.2,118.5,118.7,120.0,123.1,128.5,129.5,136.2,145.4,149.9,150.5,163.5,193.5$. LCMS (ES+) $m / z=340.3\left([\mathrm{M}+\mathrm{H}]^{+}, t_{\mathrm{R}}=1.96 \mathrm{~min}\right)$. HRMS $(\mathrm{ESI}+) m / z=340.1296[\mathrm{M}+\mathrm{H}]^{+}$found, $\mathrm{C}_{18} \mathrm{H}_{18} \mathrm{~N}_{3} \mathrm{O}_{4}{ }^{+}$ required 340.1297 .

(E)-3-(3-(2-Azidoethoxy)-4-methoxyphenyl)-1-(2-hydroxyphenyl)prop-2-en-1-one (78). A mixture of benzaldehyde 75 (3.00 g, $13.6 \mathrm{mmol})$, acetophenone 10 (1.63 mL, $13.6 \mathrm{mmol})$ and $\mathrm{KOH}(4.57 \mathrm{~g}$, $81.4 \mathrm{mmol})$ in absolute EtOH $(100 \mathrm{~mL})$ was reacted according to GP-D. The crude residue was purified by flash column chromatography $\left(\mathrm{SiO}_{2}, \mathrm{PE} / \mathrm{EtOAc} ; 5: 1\right)$ to afford chalcone $78(2.72 \mathrm{~g}, 59 \%)$ as a bright yellow powdery solid. m.p. $98-100{ }^{\circ} \mathrm{C}$. TLC $R_{\mathrm{f}}=0.33$ (PE/EtOAc; 3:1). IR $v_{\max }$ (neat) $/ \mathrm{cm}^{-1}: 3009 \mathrm{w}$ (C-H str), 2970w (C-H str), 2112m (N str), 2067w, 1738s (C=O str), 1634s, 1567s (C=C str), 1511s (C=C str), 1490s, 1440m, 1374s, 1315m, 1266s, 1227s, 1204s, 1142s, $1024 \mathrm{~s} .{ }^{1} \mathrm{H}-\mathrm{NMR}\left(500 \mathrm{MHz}, \mathrm{CDCl}_{3}\right): \delta 3.66$ $\left(2 \mathrm{H}, \mathrm{t}, J=5.0 \mathrm{~Hz},-\mathrm{OCH}_{2} \mathrm{CH}_{2} \mathrm{~N}_{3}\right), 3.89\left(3 \mathrm{H}, \mathrm{s},-\mathrm{OCH}_{3}\right), 4.22\left(2 \mathrm{H}, \mathrm{t}, J=5.0 \mathrm{~Hz},-\mathrm{OCH}_{2} \mathrm{CH}_{2} \mathrm{~N}_{3}\right), 6.90(1 \mathrm{H}, \mathrm{d}$, $J=8.5 \mathrm{~Hz}, \mathrm{ArH}), 6.91(1 \mathrm{H}, \mathrm{t}, J=8.5 \mathrm{~Hz}, \mathrm{ArH}), 7.00(1 \mathrm{H}, \mathrm{dd}, J=8.5,1.0 \mathrm{~Hz}, \mathrm{ArH}), 7.19(1 \mathrm{H}, \mathrm{d}, J=2.5 \mathrm{~Hz}$, $\mathrm{ArH}), 7.27\left(1 \mathrm{H}, \mathrm{dd}, J=8.0,2.0 \mathrm{~Hz}, \mathrm{ArH}\right.$, overlain by $\left.\mathrm{CDCl}_{3}\right), 7.46(1 \mathrm{H}, \mathrm{t}, J=7.5 \mathrm{~Hz}, \mathrm{ArH}), 7.48(1 \mathrm{H}, \mathrm{d}$, $J=15.0 \mathrm{~Hz},-\mathrm{CH}=\mathrm{CHCO}-), 7.82(1 \mathrm{H}, \mathrm{d}, J=15.0 \mathrm{~Hz},-\mathrm{CH}=\mathrm{CHCO}-), 7.90$ (1H, dd, J = 8.0, 1.5 Hz, ArH), $12.95(1 \mathrm{H}, \mathrm{s}, \mathrm{OH}) .{ }^{13} \mathrm{C}-\mathrm{NMR}\left(500 \mathrm{MHz}, \mathrm{CDCl}_{3}\right): \delta 50.1,55.8,68.2,111.7,113.4,117.7,118.4,118.6$, $119.9,124.6,127.3,129.4,136.1,145.2,147.9,152.5,163.4,193.3$. LCMS (ES+) $m / z=340.3\left([\mathrm{M}+\mathrm{H}]^{+}\right.$, $\left.t_{\mathrm{R}}=1.97 \mathrm{~min}\right)$. HRMS (ESI+) $m / z=340.1301[\mathrm{M}+\mathrm{H}]^{+}$found, $\mathrm{C}_{18} \mathrm{H}_{18} \mathrm{~N}_{3} \mathrm{O}_{4}{ }^{+}$required 340.1297.

4'-(2-Azidoethoxy)-3-hydroxyflavone (79). A mixture of chalcone 61 (2.02 g, $6.53 \mathrm{mmol}), 16 \% \mathrm{NaOH}$ $(12.9 \mathrm{~mL})$ and $15 \% \mathrm{H}_{2} \mathrm{O}_{2}(6.47 \mathrm{~mL})$ in $\mathrm{MeOH}(50 \mathrm{~mL})$ was reacted according to GP-F. The crude residue was purified by flash column chromatography $\left(\mathrm{SiO}_{2}, 1 \% \mathrm{MeOH} / \mathrm{CH}_{2} \mathrm{Cl}_{2}\right)$ to afford flavonol 79 (1.68 g, $80 \%)$ as an off-white fluffy solid. m.p. $158-160{ }^{\circ} \mathrm{C}$. TLC $R_{\mathrm{f}}=0.39\left(0.5 \% \mathrm{MeOH} / \mathrm{CH}_{2} \mathrm{Cl}_{2}\right) . \mathrm{IR} v_{\max }$ (neat) $/ \mathrm{cm}^{-1}$ : 3235w(br) (O-H str), 2957w (C-H str), 2942w (C-H str), 2140m (N $\left.\mathrm{N}_{3} \mathrm{str}\right), 2091 \mathrm{~m}, 1739 \mathrm{w}$, $1604 \mathrm{~s}$ (C=O str), 1574m (C=C str), 1512s (C=C str), 1478m, 1425m, 1406m, 1346m, 1254s, 1201s, 1182s, 1117s, 1107s, 1057s. ${ }^{1} \mathrm{H}-\mathrm{NMR}\left(500 \mathrm{MHz}, \mathrm{CDCl}_{3}\right): \delta 3.66\left(2 \mathrm{H}, \mathrm{t}, J=5.2 \mathrm{~Hz},-\mathrm{OCH}_{2} \mathrm{CH}_{2} \mathrm{~N}_{3}\right), 4.24(2 \mathrm{H}$, $\left.\mathrm{t}, J=5.2 \mathrm{~Hz},-\mathrm{OCH}_{2} \mathrm{CH}_{2} \mathrm{~N}_{3}\right), 7.03(1 \mathrm{H}$, br s, OH), $7.07(2 \mathrm{H}, \mathrm{d}, J=8.8 \mathrm{~Hz}, \mathrm{ArH}), 7.41(1 \mathrm{H}, \mathrm{t}, J=7.6 \mathrm{~Hz}$, $\mathrm{ArH}), 7.58(1 \mathrm{H}, \mathrm{d}, J=8.4 \mathrm{~Hz}, \mathrm{ArH}), 7.70(1 \mathrm{H}, \mathrm{t}, J=8.4 \mathrm{~Hz}, \mathrm{ArH}), 8.25(3 \mathrm{H}, \mathrm{d}, J=8.8 \mathrm{~Hz}, \mathrm{ArH}) .{ }^{13} \mathrm{C}-\mathrm{NMR}$ $\left(500 \mathrm{MHz}_{1} \mathrm{CDCl}_{3}\right): \delta 50.1,67.0,114.6,118.1,120.7,124.2,124.4,125.4,129.6,133.4,137.7,145.0,155.2$, 159.6, 173.1. LCMS $(\mathrm{ES}+) \mathrm{m} / z=324.1\left([\mathrm{M}+\mathrm{H}]^{+}, t_{\mathrm{R}}=1.66 \mathrm{~min}\right)$. These characterisation data are in accordance with that previously reported in the literature [51].

$4^{\prime}$-(2-Azidoethoxy)-3-hydroxy-7-methoxyflavone (80). A mixture of chalcone 74 (1.06 g, $\left.3.12 \mathrm{mmol}\right), 16 \%$ $\mathrm{NaOH}(5.89 \mathrm{~mL})$ and $15 \% \mathrm{H}_{2} \mathrm{O}_{2}(2.95 \mathrm{~mL})$ in $\mathrm{MeOH}(30 \mathrm{~mL})$ was reacted according to GP-F. The crude residue was purified by flash column chromatography $\left(\mathrm{SiO}_{2}, 1 \% \mathrm{MeOH} / \mathrm{CH}_{2} \mathrm{Cl}_{2}\right)$ to afford flavonol 80 $(677 \mathrm{mg}, 61 \%)$ as an off-white powdery solid. m.p. $172-174{ }^{\circ} \mathrm{C}$. TLC $R_{\mathrm{f}}=0.39\left(1 \% \mathrm{MeOH} / \mathrm{CH}_{2} \mathrm{Cl}_{2}\right) \cdot \mathrm{IR}$ $\nu_{\max }$ (neat) $/ \mathrm{cm}^{-1}$ : 3269w(br) (O-H str), 2932w (C-H str), 2116s ( $\left.\mathrm{N}_{3} \mathrm{str}\right), 2073 \mathrm{~m}, 1597 \mathrm{~s}(\mathrm{C}=\mathrm{O}$ str), 1561s (C=C str), 1504s (C=C str), 1451m, 1403m, 1256s, 1239s, 1203s, 1171s, 1114m, 1098m, 1050m, 1026m. ${ }^{1} \mathrm{H}-\mathrm{NMR}\left(500 \mathrm{MHz}, \mathrm{CDCl}_{3}\right): \delta 3.66\left(2 \mathrm{H}, \mathrm{t}, J=5.2 \mathrm{~Hz},-\mathrm{OCH}_{2} \mathrm{CH}_{2} \mathrm{~N}_{3}\right), 3.94\left(3 \mathrm{H}, \mathrm{s},-\mathrm{OCH}_{3}\right), 4.23(2 \mathrm{H}, \mathrm{t}$, $\left.J=5.2 \mathrm{~Hz},-\mathrm{OCH}_{2} \mathrm{CH}_{2} \mathrm{~N}_{3}\right), 6.94(1 \mathrm{H}, \mathrm{d}, J=2.0 \mathrm{~Hz}, \mathrm{ArH}), 6.98(1 \mathrm{H}, \mathrm{dd}, J=8.8,2.0 \mathrm{~Hz}, \mathrm{ArH}), 7.04(1 \mathrm{H}$, br s, OH), $7.05(2 \mathrm{H}, \mathrm{d}, J=9.2 \mathrm{~Hz}, \mathrm{ArH}), 8.12(1 \mathrm{H}, \mathrm{d}, J=8.8 \mathrm{~Hz}, \mathrm{ArH}), 8.21(2 \mathrm{H}, \mathrm{d}, J=8.8 \mathrm{~Hz}, \mathrm{ArH})$. ${ }^{13} \mathrm{C}-\mathrm{NMR}\left(500 \mathrm{MHz}, \mathrm{CDCl}_{3}\right): \delta 50.1,55.8,67.0,99.8,114.5,114.6,114.7,124.3,126.6,129.2,137.4,144.3$, 157.1, 159.3, 164.1, 172.6. LCMS (ES+) $m / z=354.2\left([\mathrm{M}+\mathrm{H}]^{+}, t_{\mathrm{R}}=1.90 \mathrm{~min}\right)$. HRMS (ESI+) $m / z=$ $354.1074[\mathrm{M}+\mathrm{H}]^{+}$found, $\mathrm{C}_{18} \mathrm{H}_{16} \mathrm{O}_{5} \mathrm{~N}_{3}{ }^{+}$required 354.1084 .

4'-(2-Azidoethoxy)-3-hydroxy-3'-methoxyflavone (81). A mixture of chalcone 77 (519 mg, $1.53 \mathrm{mmol}$ ), $16 \% \mathrm{NaOH}(2.95 \mathrm{~mL})$ and $15 \% \mathrm{H}_{2} \mathrm{O}_{2}(1.47 \mathrm{~mL})$ in $\mathrm{MeOH}(20 \mathrm{~mL})$ was reacted according to GP-F. The crude residue was purified by flash column chromatography $\left(\mathrm{SiO}_{2}, 1 \% \mathrm{MeOH} / \mathrm{CH}_{2} \mathrm{Cl}_{2}\right)$ to afford flavonol 81 (418 mg, 77\%) as a pale yellow-white powdery solid. m.p. 118-120 ${ }^{\circ} \mathrm{C}$. TLC $R_{\mathrm{f}}=0.45$ $\left(0.5 \% \mathrm{MeOH} / \mathrm{CH}_{2} \mathrm{Cl}_{2}\right)$. IR $v_{\max }$ (neat) $/ \mathrm{cm}^{-1}$ : 3224w(br) $\left(\mathrm{O}-\mathrm{H}\right.$ str), 2924w (C-H str), 2125s ( $\left.\mathrm{N}_{3} \mathrm{str}\right)$, 1614 s (C=O str), 1567m (C=C str), 1514s (C=C str), 1481m, 1470m, 1409s, 1269s, 1202s, 1183m, 1146s, 
$1121 \mathrm{~m}, 1035 \mathrm{~m}, 1006 \mathrm{~m} .{ }^{1} \mathrm{H}-\mathrm{NMR}\left(500 \mathrm{MHz} \mathrm{CDCl}_{3}\right): \delta 3.71\left(2 \mathrm{H}, \mathrm{t}, \mathrm{J}=5.2 \mathrm{~Hz},-\mathrm{OCH}_{2} \mathrm{CH}_{2} \mathrm{~N}_{3}\right), 3.99(3 \mathrm{H}$, $\left.\mathrm{s},-\mathrm{OCH}_{3}\right), 4.29\left(2 \mathrm{H}, \mathrm{t}, J=5.2 \mathrm{~Hz},-\mathrm{OCH}_{2} \mathrm{CH}_{2} \mathrm{~N}_{3}\right), 7.01(1 \mathrm{H}$, br s, OH), $7.05(1 \mathrm{H}, \mathrm{d}, J=9.2 \mathrm{~Hz}, \mathrm{ArH})$, $7.43(1 \mathrm{H}, \mathrm{t}, J=7.2 \mathrm{~Hz}, \mathrm{ArH}), 7.60(1 \mathrm{H}, \mathrm{d}, J=8.4 \mathrm{~Hz}, \mathrm{ArH}), 7.72(1 \mathrm{H}, \mathrm{t}, J=8.4 \mathrm{~Hz}, \mathrm{ArH}), 7.87-7.89(2 \mathrm{H}$, $\mathrm{m}, \mathrm{ArH}), 8.26(1 \mathrm{H}, \mathrm{dd}, J=8.0,1.2 \mathrm{~Hz}, \mathrm{ArH}) .{ }^{13} \mathrm{C}-\mathrm{NMR}\left(500 \mathrm{MHz}, \mathrm{CDCl}_{3}\right): \delta 50.1,56.2,67.9,111.6$, $113.3,118.2,120.6,121.3,124.5,124.8,125.4,133.5,137.9,144.8,149.5,149.5,155.3,173.2$. LCMS (ES+) $m / z=354.2\left([\mathrm{M}+\mathrm{H}]^{+}, t_{\mathrm{R}}=1.86 \mathrm{~min}\right) . \operatorname{HRMS}(\mathrm{ESI}+) m / z=354.1095[\mathrm{M}+\mathrm{H}]^{+}$found, $\mathrm{C}_{18} \mathrm{H}_{16} \mathrm{~N}_{3} \mathrm{O}_{5}{ }^{+}$ required 354.1090 .

3'-(2-Azidoethoxy)-3-hydroxy-4'-methoxyflavone (82). A mixture of chalcone 78 (513 $\mathrm{mg}, 1.51 \mathrm{mmol}$ ), $16 \% \mathrm{NaOH}(2.95 \mathrm{~mL})$ and $15 \% \mathrm{H}_{2} \mathrm{O}_{2}(1.47 \mathrm{~mL})$ in $\mathrm{MeOH}(20 \mathrm{~mL})$ was reacted according to GP-F. The crude residue was purified by flash column chromatography $\left(\mathrm{SiO}_{2}, 1 \% \mathrm{MeOH} / \mathrm{CH}_{2} \mathrm{Cl}_{2}\right)$ to afford flavonol $82(419 \mathrm{mg}, 78 \%)$ as a pale yellow-white powdery solid. m.p. $164-166{ }^{\circ} \mathrm{C}$. TLC $R_{\mathrm{f}}=0.31(0.5 \%$ $\mathrm{MeOH} / \mathrm{CH}_{2} \mathrm{Cl}_{2}$ ). IR $v_{\max }$ (neat) $/ \mathrm{cm}^{-1}: 3199 \mathrm{~m}(\mathrm{br})(\mathrm{O}-\mathrm{H}$ str), 2974w (C-H str), 2942w (C-H str), 2105s ( $\mathrm{N}_{3}$ str), 1611s (C=O str), 1599m (C=O str), 1564s (C=C str), 1516s (C=C str), 1480s, 1463m, 1412s, 1350m, 1264s, 1203s, 1179s, 1141s, 1012s. ${ }^{1} \mathrm{H}-\mathrm{NMR}\left(500 \mathrm{MHz}, \mathrm{CDCl}_{3}\right): \delta 3.71\left(2 \mathrm{H}, \mathrm{t}, J=5.2 \mathrm{~Hz},-\mathrm{OCH}_{2} \mathrm{CH}_{2} \mathrm{~N}_{3}\right)$, $3.97\left(3 \mathrm{H}, \mathrm{s},-\mathrm{OCH}_{3}\right), 4.31\left(2 \mathrm{H}, \mathrm{t}, \mathrm{J}=5.2 \mathrm{~Hz},-\mathrm{OCH}_{2} \mathrm{CH}_{2} \mathrm{~N}_{3}\right), 7.01(1 \mathrm{H}, \mathrm{br} \mathrm{s}, \mathrm{OH}), 7.06(1 \mathrm{H}, \mathrm{d}, J=8.4 \mathrm{~Hz}$, $\operatorname{ArH}), 7.43(1 \mathrm{H}, \mathrm{t}, J=7.6 \mathrm{~Hz}, \mathrm{ArH}), 7.60(1 \mathrm{H}, \mathrm{d}, J=8.4 \mathrm{~Hz}, \mathrm{ArH}), 7.71(1 \mathrm{H}, \mathrm{t}, J=8.4 \mathrm{~Hz}, \mathrm{ArH}), 7.91(1 \mathrm{H}$, $\mathrm{d}, J=2.0 \mathrm{~Hz}, \mathrm{ArH}), 7.96(1 \mathrm{H}, \mathrm{dd}, J=8.8,2.0 \mathrm{~Hz}, \mathrm{ArH}), 8.26(1 \mathrm{H}, \mathrm{dd}, J=8.0,1.2 \mathrm{~Hz}, \mathrm{ArH}) .{ }^{13} \mathrm{C}-\mathrm{NMR}$ $\left(500 \mathrm{MHz}_{2} \mathrm{CDCl}_{3}\right): \delta 50.2,56.0,68.3,111.7,113.8,118.2,120.6,122.6,123.7,124.5,125.4,133.5,137.8$, $144.8,147.6,151.6,155.2,173.1$. LCMS (ES+) $m / z=354.2\left([\mathrm{M}+\mathrm{H}]^{+}, t_{\mathrm{R}}=1.86 \mathrm{~min}\right)$. HRMS (ESI+) $m / z=354.1096[\mathrm{M}+\mathrm{H}]^{+}$found, $\mathrm{C}_{18} \mathrm{H}_{16} \mathrm{~N}_{3} \mathrm{O}_{5}{ }^{+}$required 354.1090.

6-(2-Bromoethoxy)flavone (83). A mixture of flavone 28 (1.02 g, $4.28 \mathrm{mmol}), 1$,2-dibromoethane (0.47 $\mathrm{mL}$, $5.46 \mathrm{mmol})$ and anhydrous $\mathrm{K}_{2} \mathrm{CO}_{3}(782 \mathrm{mg}, 5.66 \mathrm{mmol})$ in dry acetone $(50 \mathrm{~mL})$ was reacted according to GP-I. The crude residue was purified by flash column chromatography $\left(\mathrm{SiO}_{2}, \mathrm{CH}_{2} \mathrm{Cl}_{2}\right)$ to afford flavone $83(328 \mathrm{mg}, 22 \%)$ as a white powdery solid. m.p. $180-182{ }^{\circ} \mathrm{C}$. TLC $R_{\mathrm{f}}=0.33\left(1 \% \mathrm{MeOH} / \mathrm{CH}_{2} \mathrm{Cl}_{2}\right)$. IR $v_{\max }$ (neat)/cm ${ }^{-1}$ : 3026w (C-H str), 2938w (C-H str), 2887w (C-H str), 1637s (C=O str), 1617m, $1586 \mathrm{~m}(\mathrm{C}=\mathrm{C}$ str), 1568m (C=C str), 1482m, 1468m, 1447s, 1358s, 1313w, 1254m, 1200m, 1084m, 1030m, 1017s. ${ }^{1} \mathrm{H}-\mathrm{NMR}\left(500 \mathrm{MHz}, \mathrm{CDCl}_{3}\right): \delta 3.69\left(2 \mathrm{H}, \mathrm{t}, J=6.0 \mathrm{~Hz},-\mathrm{OCH}_{2} \mathrm{CH}_{2} \mathrm{Br}\right), 4.41(2 \mathrm{H}, \mathrm{t}, J=6.0 \mathrm{~Hz}$, $\left.-\mathrm{OCH}_{2} \mathrm{CH}_{2} \mathrm{Br}\right), 6.82(1 \mathrm{H}, \mathrm{s},-\mathrm{C}=\mathrm{CH}), 7.34(1 \mathrm{H}, \mathrm{dd}, J=9.2,3.2 \mathrm{~Hz}, \mathrm{ArH}), 7.50-7.54(4 \mathrm{H}, \mathrm{m}, \mathrm{ArH}), 7.58$ $(1 \mathrm{H}, \mathrm{d}, J=3.2 \mathrm{~Hz}, \mathrm{ArH}), 7.91-7.93(2 \mathrm{H}, \mathrm{m}, \mathrm{ArH}) .{ }^{13} \mathrm{C}-\mathrm{NMR}\left(500 \mathrm{MHz}, \mathrm{CDCl}_{3}\right): \delta 28.9,68.4,105.9$, $106.8,119.8,124.1,124.5,126.2,129.0,131.5,131.7,151.3,155.4,163.2,178.1$. LCMS (ES+) $\mathrm{m} / z=347.0$ $\left([\mathrm{M}+\mathrm{H}]^{+}, t_{\mathrm{R}}=1.66 \mathrm{~min}\right)$. These characterisation data are in accordance with that previously reported in the literature [54].

7-(2-Bromoethoxy)flavone (84). A mixture of flavone 29 (1.59 g, $6.67 \mathrm{mmol}), 1$,2-dibromoethane (0.71 $\mathrm{mL}$, $8.19 \mathrm{mmol})$ and anhydrous $\mathrm{K}_{2} \mathrm{CO}_{3}(1.20 \mathrm{~g}, 8.68 \mathrm{mmol})$ in dry acetone $(50 \mathrm{~mL})$ was reacted according to GP-I. The crude residue was purified by flash column chromatography $\left(\mathrm{SiO}_{2}, 1 \% \mathrm{MeOH} / \mathrm{CH}_{2} \mathrm{Cl}_{2}\right)$ to afford flavone $84(1.50 \mathrm{~g}, 65 \%)$ as a white powdery solid. m.p. $150-152{ }^{\circ} \mathrm{C}$. TLC $R_{\mathrm{f}}=0.22(1 \%$ $\mathrm{MeOH} / \mathrm{CH}_{2} \mathrm{Cl}_{2}$ ). IR $v_{\max }$ (neat) $/ \mathrm{cm}^{-1}$ : 3045w (C-H str), 2934w (C-H str), 2864w (C-H str), 1628s (C=O str), 1604s, 1594s (C=C str), 1567m (C=C str), 1494m, 1439m, 1372s, 1356s, 1282s, 1250s, 1227s, 1171s, $1131 \mathrm{~m}, 1089 \mathrm{~s}, 1013 \mathrm{~m} .{ }^{1} \mathrm{H}-\mathrm{NMR}\left(500 \mathrm{MHz}, \mathrm{CDCl}_{3}\right): \delta 3.70\left(2 \mathrm{H}, \mathrm{t}, \mathrm{J}=6.0 \mathrm{~Hz},-\mathrm{OCH}_{2} \mathrm{CH}_{2} \mathrm{Br}\right), 4.41(2 \mathrm{H}$, $\left.\mathrm{t}, J=6.0 \mathrm{~Hz},-\mathrm{OCH}_{2} \mathrm{CH}_{2} \mathrm{Br}\right), 6.76(1 \mathrm{H}, \mathrm{s},-\mathrm{C}=\mathrm{CH}), 6.97(1 \mathrm{H}, \mathrm{d}, J=2.4 \mathrm{~Hz}, \mathrm{ArH}), 7.00(1 \mathrm{H}, \mathrm{dd}, J=8.8$, $2.4 \mathrm{~Hz}, \mathrm{ArH}), 7.49-7.54$ (3H, m, ArH), 7.88-7.91 (2H, m, ArH), $8.14(1 \mathrm{H}, \mathrm{d}, J=8.8 \mathrm{~Hz}, \mathrm{ArH}) .{ }^{13} \mathrm{C}-\mathrm{NMR}$ $\left(500 \mathrm{MHz}_{1} \mathrm{CDCl}_{3}\right): \delta 28.3,68.2,101.3,107.5,114.4,118.3,126.1,127.3,129.0,131.4,131.7,157.8,162.4$, 163.0, 177.7. LCMS (ES+) $m / z=347.0\left([\mathrm{M}+\mathrm{H}]^{+}, t_{\mathrm{R}}=1.69 \mathrm{~min}\right)$. These characterisation data are in accordance with that previously reported in the literature [28].

6-(2-Azidoethoxy)flavone (85). A mixture of flavone 83 (824 mg, $2.39 \mathrm{mmol})$ and $\mathrm{NaN}_{3}$ (336 mg, $5.17 \mathrm{mmol})$ in dry DMF $(20 \mathrm{~mL})$ was reacted according to GP-J. The reaction mixture was worked up to afford flavone 85 (686 mg, 93\%) as a white powdery solid and was used without further purification. m.p. $138-140{ }^{\circ} \mathrm{C}$. TLC $R_{\mathrm{f}}=0.27\left(1 \% \mathrm{MeOH} / \mathrm{CH}_{2} \mathrm{Cl}_{2}\right)$. IR $v_{\max }$ (neat) $/ \mathrm{cm}^{-1}$ : 3073w (C-H str), 2930w 
(C-H str), 2107s ( $\mathrm{N}_{3}$ str), 2063m, 1625s, 1616s (C=O str), 1603s, 1582s (C=C str), 1569s (C=C str), 1480s, 1455s, 1363s, 1291s, 1262m, 1242s, 1203s, 1130s, 1083m. ${ }^{1} \mathrm{H}-\mathrm{NMR}\left(500 \mathrm{MHz}, \mathrm{CDCl}_{3}\right): \delta 3.65(2 \mathrm{H}, \mathrm{t}$, $\left.J=4.8 \mathrm{~Hz},-\mathrm{OCH}_{2} \mathrm{CH}_{2} \mathrm{~N}_{3}\right), 4.26\left(2 \mathrm{H}, \mathrm{t}, J=4.8 \mathrm{~Hz},-\mathrm{OCH}_{2} \mathrm{CH}_{2} \mathrm{~N}_{3}\right), 6.81(1 \mathrm{H}, \mathrm{s},-\mathrm{C}=\mathrm{CH}), 7.34(1 \mathrm{H}, \mathrm{dd}$, $J=8.8,2.8 \mathrm{~Hz}, \mathrm{ArH}), 7.51-7.54(4 \mathrm{H}, \mathrm{m}, \mathrm{ArH}), 7.59(1 \mathrm{H}, \mathrm{d}, J=2.8 \mathrm{~Hz}, \mathrm{ArH}), 7.90-7.93(2 \mathrm{H}, \mathrm{m}, \mathrm{ArH})$. ${ }^{13} \mathrm{C}-\mathrm{NMR}\left(500 \mathrm{MHz}, \mathrm{CDCl}_{3}\right): \delta 50.0,67.5,105.6,106.9,119.8,124.2,124.5,126.2,129.0,131.5,131.8,151.3$, 155.5, 163.3, 178.1. LCMS (ES+) $m / z=308.2\left([\mathrm{M}+\mathrm{H}]^{+}, t_{\mathrm{R}}=1.68 \mathrm{~min}\right.$ ). HRMS (ESI+) $m / z=330.0836$ $[\mathrm{M}+\mathrm{Na}]^{+}$found, $\mathrm{C}_{17} \mathrm{H}_{13} \mathrm{O}_{3} \mathrm{~N}_{3} \mathrm{Na}^{+}$required 330.0849 .

7-(2-Azidoethoxy)flavone (86). A mixture of flavone 84 (1.03 g, $3.00 \mathrm{mmol})$ and $\mathrm{NaN}_{3}(444 \mathrm{mg}, 6.83 \mathrm{mmol})$ in dry DMF $(20 \mathrm{~mL})$ was reacted according to GP-J. The reaction mixture was worked up to afford flavone $86(838 \mathrm{mg}, 91 \%)$ as an off-white powdery solid and was used without further purification. m.p. $108-110^{\circ} \mathrm{C}$. TLC $R_{\mathrm{f}}=0.23\left(1 \% \mathrm{MeOH} / \mathrm{CH}_{2} \mathrm{Cl}_{2}\right)$. IR $v_{\max }$ (neat) $/ \mathrm{cm}^{-1}: 3073 \mathrm{w}(\mathrm{C}-\mathrm{H} \mathrm{str}), 2979 \mathrm{w}$ (C-H str), 2926w (C-H str), 2105m ( $\mathrm{N}_{3}$ str), 1631s (C=O str), 1601s, $1578 \mathrm{~m}$ (C=C str), $1569 \mathrm{~m}$ (C=C str), $1493 \mathrm{w}, 1447 \mathrm{~s}, 1375 \mathrm{~m}, 1358 \mathrm{~m}, 1303 \mathrm{~m}, 1244 \mathrm{~s}, 1177 \mathrm{~s}, 1130 \mathrm{~m}, 1085 \mathrm{~s} .{ }^{1} \mathrm{H}-\mathrm{NMR}\left(500 \mathrm{MHz}, \mathrm{CDCl}_{3}\right): \delta 3.68$ $\left(2 \mathrm{H}, \mathrm{t}, J=5.0 \mathrm{~Hz},-\mathrm{OCH}_{2} \mathrm{CH}_{2} \mathrm{~N}_{3}\right), 4.26\left(2 \mathrm{H}, \mathrm{t}, J=5.0 \mathrm{~Hz},-\mathrm{OCH}_{2} \mathrm{CH}_{2} \mathrm{~N}_{3}\right), 6.75(1 \mathrm{H}, \mathrm{s},-\mathrm{C}=\mathrm{CH}), 6.97(1 \mathrm{H}$, $\mathrm{d}, J=2.0 \mathrm{~Hz}, \mathrm{ArH}), 6.99(1 \mathrm{H}, \mathrm{dd}, J=8.5,2.0 \mathrm{~Hz}, \mathrm{ArH}), 7.48-7.54(3 \mathrm{H}, \mathrm{m}, \mathrm{ArH}), 7.88(2 \mathrm{H}, \mathrm{dd}, J=8.0$, $2.0 \mathrm{~Hz}, \mathrm{ArH}), 8.13(1 \mathrm{H}, \mathrm{d}, J=8.5 \mathrm{~Hz}, \mathrm{ArH}) .{ }^{13} \mathrm{C}-\mathrm{NMR}\left(500 \mathrm{MHz}, \mathrm{CDCl}_{3}\right): \delta 49.8,67.4,101.3,107.5,114.3$, $118.2,126.1,127.2,128.9,131.4,131.6,157.7,162.6,163.0,177.6$. LCMS (ES+) $m / z=308.0\left([\mathrm{M}+\mathrm{H}]^{+}\right.$, $\left.t_{\mathrm{R}}=4.43 \mathrm{~min}\right)$. These characterisation data are in accordance with that previously reported in the literature [10].

4'-(2-Azidoethoxy)-7-methoxyflavone (87). To a stirred solution of chalcone 76 (1.02 g, $3.01 \mathrm{mmol})$ in DMSO $(20 \mathrm{~mL})$ was added a catalytic amount of $\mathrm{I}_{2}(103 \mathrm{mg}, 0.407 \mathrm{mmol})$. The reaction mixture was heated at $130^{\circ} \mathrm{C}$ with stirring for $24 \mathrm{~h}$ under a nitrogen atmosphere. The resulting mixture was allowed to cool to room temperature and poured into $\mathrm{H}_{2} \mathrm{O}(50 \mathrm{~mL})$. The aqueous solution was extracted with $\mathrm{CHCl}_{3}(2 \times 100 \mathrm{~mL})$ and the combined organic layer was washed with $\mathrm{H}_{2} \mathrm{O}(2 \times 50 \mathrm{~mL})$, brine $(2 \times 50 \mathrm{~mL})$, dried over anhydrous $\mathrm{MgSO}_{4}$, filtered and the solvent removed under reduced pressure. The crude residue was purified by flash column chromatography $\left(\mathrm{SiO}_{2}, 1 \% \mathrm{MeOH} / \mathrm{CH}_{2} \mathrm{Cl}_{2}\right)$ to afford flavone $87(924 \mathrm{mg}, 91 \%)$ as a pale yellow-white powdery solid. m.p. $128-130{ }^{\circ} \mathrm{C}$. TLC $R_{\mathrm{f}}=0.35(3 \%$ $\mathrm{MeOH} / \mathrm{CH}_{2} \mathrm{Cl}_{2}$ ). IR $v_{\max }$ (neat) $/ \mathrm{cm}^{-1}$ : 2946w (C-H str), 2839w (C-H str), 2097s ( $\left.\mathrm{N}_{3} \mathrm{str}\right), 1644 \mathrm{~s}(\mathrm{C}=\mathrm{O}$ str), 1626s, 1598s (C=C str), 1510s (C=C str), 1503s (C=C str), 1440s, 1376m, 1355s, 1247s, 1182s, 1164m, $1141 \mathrm{w}, 1091 \mathrm{~m}, 1023 \mathrm{~m} .{ }^{1} \mathrm{H}-\mathrm{NMR}\left(500 \mathrm{MHz}, \mathrm{CDCl}_{3}\right): \delta 3.65\left(2 \mathrm{H}, \mathrm{t}, J=5.2 \mathrm{~Hz},-\mathrm{OCH}_{2} \mathrm{CH}_{2} \mathrm{~N}_{3}\right), 3.92(3 \mathrm{H}, \mathrm{s}$, $\left.-\mathrm{OCH}_{3}\right), 4.22\left(2 \mathrm{H}, \mathrm{t}, J=5.2 \mathrm{~Hz},-\mathrm{OCH}_{2} \mathrm{CH}_{2} \mathrm{~N}_{3}\right), 6.66(1 \mathrm{H}, \mathrm{s},-\mathrm{C}=\mathrm{CH}), 6.94(1 \mathrm{H}, \mathrm{d}, J=2.4 \mathrm{~Hz}, \mathrm{ArH}), 6.96$ $(1 \mathrm{H}, \mathrm{dd}, J=8.8,2.4 \mathrm{~Hz}, \mathrm{ArH}), 7.02(2 \mathrm{H}, \mathrm{d}, J=9.2 \mathrm{~Hz}, \mathrm{ArH}), 7.85(2 \mathrm{H}, \mathrm{d}, J=9.2 \mathrm{~Hz}, \operatorname{ArH}), 8.11(1 \mathrm{H}, \mathrm{d}$, $J=8.8 \mathrm{~Hz}, \mathrm{ArH}) .{ }^{13} \mathrm{C}-\mathrm{NMR}\left(500 \mathrm{MHz}, \mathrm{CDCl}_{3}\right): \delta 50.0,55.8,67.1,100.3,106.2,114.2,114.9,117.7,124.7$, 126.9, 127.8, 157.8, 160.7, 162.7, 164.0, 177.7. LCMS (ES+) $m / z=338.2\left([\mathrm{M}+\mathrm{H}]^{+}, t_{\mathrm{R}}=1.84 \mathrm{~min}\right)$. HRMS (ESI+) $m / z=338.1147[\mathrm{M}+\mathrm{H}]^{+}$found, $\mathrm{C}_{18} \mathrm{H}_{16} \mathrm{~N}_{3} \mathrm{O}_{4}{ }^{+}$required 338.1141.

4'-(2-Azidoethoxy)aurone (88). A mixture of chalcone 61 (403 mg, $1.30 \mathrm{mmol})$ and $\mathrm{Hg}(\mathrm{OAc})_{2}(440 \mathrm{mg}$, $1.38 \mathrm{mmol})$ in dry pyridine $(15 \mathrm{~mL})$ was reacted according to GP-K. The crude residue was purified by flash column chromatography $\left(\mathrm{SiO}_{2}, \mathrm{PE} / \mathrm{EtOAc} ; 3: 1\right)$ to afford aurone 88 (354 $\left.\mathrm{mg}, 88 \%\right)$ as a bright yellow powdery solid. m.p. $114-116{ }^{\circ} \mathrm{C}$. TLC $R_{\mathrm{f}}=0.37$ (PE/EtOAc; $2: 1$ ). IR $v_{\max }$ (neat) $/ \mathrm{cm}^{-1}: 2945 \mathrm{w}$ (C-H str), 2888w (C-H str), 2096s ( $\mathrm{N}_{3}$ str), 2062m, 1699s (C=O str), 1649s, 1592s (C=C str), 1510m (C=C str), 1474m, 1461m, 1395w, 1347m, 1301s, 1257s, 1177s, 1130m, 1111m, 1097w, 1048s, $1009 \mathrm{w} .{ }^{1} \mathrm{H}-\mathrm{NMR}$ $\left(500 \mathrm{MHz}, \mathrm{CDCl}_{3}\right): \delta 3.65\left(2 \mathrm{H}, \mathrm{t}, J=4.8 \mathrm{~Hz},-\mathrm{OCH}_{2} \mathrm{CH}_{2} \mathrm{~N}_{3}\right), 4.22\left(2 \mathrm{H}, \mathrm{t}, J=4.8 \mathrm{~Hz},-\mathrm{OCH}_{2} \mathrm{CH}_{2} \mathrm{~N}_{3}\right), 6.89$ $(1 \mathrm{H}, \mathrm{s},-\mathrm{C}=\mathrm{CH}), 7.01(2 \mathrm{H}, \mathrm{d}, J=8.8 \mathrm{~Hz}, \mathrm{ArH}), 7.22(1 \mathrm{H}, \mathrm{t}, J=7.6 \mathrm{~Hz}, \mathrm{ArH}), 7.33(1 \mathrm{H}, \mathrm{d}, J=8.4 \mathrm{~Hz}, \mathrm{ArH})$, $7.65(1 \mathrm{H}, \mathrm{t}, J=8.4 \mathrm{~Hz}, \mathrm{ArH}), 7.82(1 \mathrm{H}, \mathrm{dd}, J=7.6,0.4 \mathrm{~Hz}, \mathrm{ArH}), 7.91(2 \mathrm{H}, \mathrm{d}, J=8.8 \mathrm{~Hz}, \mathrm{ArH}) .{ }^{13} \mathrm{C}-\mathrm{NMR}$ (500 MHz, $\left.\mathrm{CDCl}_{3}\right): \delta 50.0,67.0,112.9,113.0,115.0,121.8,123.3,124.6,125.7,133.4,136.6,146.0,159.6$, 165.9, 184.6. LCMS (ES+) $m / z=308.2\left([\mathrm{M}+\mathrm{H}]^{+}, t_{\mathrm{R}}=1.73 \mathrm{~min}\right)$. HRMS (ESI+) $m / z=308.1035[\mathrm{M}+\mathrm{H}]^{+}$ found, $\mathrm{C}_{17} \mathrm{H}_{14} \mathrm{O}_{3} \mathrm{~N}_{3}{ }^{+}$required 308.1035 . 
4'-(2-Azidoethoxy)-6-methoxyaurone (89). A mixture of chalcone $76(2.01 \mathrm{~g}, 5.91 \mathrm{mmol})$ and $\mathrm{Hg}(\mathrm{OAc})_{2}$ $(1.95 \mathrm{~g}, 6.13 \mathrm{mmol})$ in dry pyridine $(50 \mathrm{~mL})$ was reacted according to GP-K. The crude residue was purified by flash column chromatography $\left(\mathrm{SiO}_{2}, 0.5 \% \mathrm{MeOH} / \mathrm{CH}_{2} \mathrm{Cl}_{2}\right)$ to afford aurone $89(1.91 \mathrm{~g}$, $96 \%)$ as a bright yellow-orange powdery solid. m.p. $108-110{ }^{\circ} \mathrm{C}$. TLC $R_{\mathrm{f}}=0.36\left(0.5 \% \mathrm{MeOH} / \mathrm{CH}_{2} \mathrm{Cl}_{2}\right)$. IR $v_{\max }$ (neat) $/ \mathrm{cm}^{-1}: 3008 \mathrm{w}$ (C-H str), 2970w (C-H str), 2092s ( $\left.\mathrm{N}_{3} \mathrm{str}\right), 1694 \mathrm{~m}$ (C=O str), 1652m, 1594s $(C=C$ str), $1570 \mathrm{~m}(\mathrm{C}=\mathrm{C}$ str), 1512m (C=C str), 1443m, 1414w, 1349m, 1315w, 1271m, 1248s, 1185m, 1131s, 1110s, 1068w, 1035m. ${ }^{1} \mathrm{H}-\mathrm{NMR}\left(500 \mathrm{MHz}, \mathrm{CDCl}_{3}\right): \delta 3.63\left(2 \mathrm{H}, \mathrm{t}, J=4.8 \mathrm{~Hz},-\mathrm{OCH}_{2} \mathrm{CH}_{2} \mathrm{~N}_{3}\right), 3.91(3 \mathrm{H}, \mathrm{s}$, $\left.-\mathrm{OCH}_{3}\right), 4.19\left(2 \mathrm{H}, \mathrm{t}, J=4.8 \mathrm{~Hz},-\mathrm{OCH}_{2} \mathrm{CH}_{2} \mathrm{~N}_{3}\right), 6.72-6.74(2 \mathrm{H}, \mathrm{m}, \mathrm{ArH}), 6.76(1 \mathrm{H}, \mathrm{s},-\mathrm{C}=\mathrm{CH}), 6.97(2 \mathrm{H}$, $\mathrm{d}, J=8.8 \mathrm{~Hz}, \mathrm{ArH}), 7.68(1 \mathrm{H}, \mathrm{d}, J=8.8 \mathrm{~Hz}, \mathrm{ArH}), 7.83(2 \mathrm{H}, \mathrm{d}, J=8.8 \mathrm{~Hz}, \mathrm{ArH}) .{ }^{13} \mathrm{C}-\mathrm{NMR}(500 \mathrm{MHz}$, $\mathrm{CDCl}_{3}$ ): $\delta 50.0,55.9,66.9,96.5,111.7,112.0,114.9,115.0,125.6,125.7,133.1,146.8,159.3,167.2,168.2$, 182.8. LCMS (ES+) $m / z=338.2\left([\mathrm{M}+\mathrm{H}]^{+}, t_{\mathrm{R}}=1.65 \mathrm{~min}\right)$. HRMS (ESI+) $m / z=338.1146[\mathrm{M}+\mathrm{H}]^{+}$ found, $\mathrm{C}_{18} \mathrm{H}_{16} \mathrm{~N}_{3} \mathrm{O}_{4}{ }^{+}$required 338.1141.

\subsection{Synthesis of Triazole-Bridged Flavonoid Dimers and Trimers}

(E)-3-(3,4-Dimethoxyphenyl)-1-(3-methoxy-4-(2-(4-((2-methoxy-4-((E)-3-(4-methoxyphenyl)acryloyl))methyl)1H-1,2,3-triazol-1-yl)ethoxy)phenyl)prop-2-en-1-one (90). A mixture of alkyne chalcone 20 (254 mg, $0.789 \mathrm{mmol})$, azide chalcone 67 ( $305 \mathrm{mg}, 0.796 \mathrm{mmol}), \mathrm{CuSO}_{4} \cdot 5 \mathrm{H}_{2} \mathrm{O}(225 \mathrm{mg}, 0.900 \mathrm{mmol})$ and sodium ascorbate $(389 \mathrm{mg}, 1.97 \mathrm{mmol})$ in $t-\mathrm{BuOH} / \mathrm{H}_{2} \mathrm{O}(1: 1,40 \mathrm{~mL})$ was reacted according to GP-A. The crude residue was purified by flash column chromatography $\left(\mathrm{SiO}_{2}, 1 \%-5 \% \mathrm{MeOH} / \mathrm{CH}_{2} \mathrm{Cl}_{2}\right)$ and recrystallized from $\mathrm{MeOH}$ to afford triazole hybrid $90(151 \mathrm{mg}, 27 \%)$ as a pale yellow-brown powdery solid. m.p. $108-110{ }^{\circ} \mathrm{C}$. TLC $R_{\mathrm{f}}=0.41\left(5 \% \mathrm{MeOH} / \mathrm{CH}_{2} \mathrm{Cl}_{2}\right)$. IR $v_{\max }$ (neat) $/ \mathrm{cm}^{-1}: 2942 \mathrm{w}$ (C-H str), $2837 \mathrm{w}$ (C-H str), 1652m (C=O str), 1595m (C=C str), 1572m (C=C str), 1509s (C=C str), 1464m, 1420m, 1335w, 1307w, 1255s, 1195w, 1143s, 1021s. ${ }^{1} \mathrm{H}-\mathrm{NMR}\left(500 \mathrm{MHz}, \mathrm{CDCl}_{3}\right): \delta 3.86\left(3 \mathrm{H}, \mathrm{s},-\mathrm{OCH}_{3}\right), 3.92$ $\left(3 \mathrm{H}, \mathrm{s},-\mathrm{OCH}_{3}\right), 3.94\left(3 \mathrm{H}, \mathrm{s},-\mathrm{OCH}_{3}\right), 3.95\left(3 \mathrm{H}, \mathrm{s},-\mathrm{OCH}_{3}\right), 3.96\left(3 \mathrm{H}, \mathrm{s},-\mathrm{OCH}_{3}\right), 4.46(2 \mathrm{H}, \mathrm{t}, J=5.2 \mathrm{~Hz}$, $\left.-\mathrm{OCH}_{2} \mathrm{CH}_{2} \mathrm{~N}-\right), 4.84\left(2 \mathrm{H}, \mathrm{t}, J=5.2 \mathrm{~Hz},-\mathrm{OCH}_{2} \mathrm{CH}_{2} \mathrm{~N}-\right), 5.40\left(2 \mathrm{H}, \mathrm{s},-\mathrm{OCH}_{2} \mathrm{CN}-\right), 6.82(1 \mathrm{H}, \mathrm{d}, J=8.0 \mathrm{~Hz}$, $\mathrm{ArH}), 6.90(1 \mathrm{H}, \mathrm{d}, J=8.4 \mathrm{~Hz}, \mathrm{ArH}), 6.93(2 \mathrm{H}, \mathrm{d}, J=8.8 \mathrm{~Hz}, \mathrm{ArH}), 7.14-7.17(2 \mathrm{H}, \mathrm{m}, \mathrm{ArH}), 7.24(1 \mathrm{H}, \mathrm{dd}$, $J=8.4,2.0 \mathrm{~Hz}, \mathrm{ArH}), 7.37(1 \mathrm{H}, \mathrm{d}, J=15.2 \mathrm{~Hz},-\mathrm{CH}=\mathrm{CHCO}-), 7.41(1 \mathrm{H}, \mathrm{d}, J=15.6 \mathrm{~Hz},-\mathrm{CH}=\mathrm{CHCO}-)$, 7.57-7.62 (6H, m, ArH), 7.75 (1H, d, J = 15.6 Hz, -CH=CHCO-), 7.77 (1H, d, J = 15.6 Hz, -CH=CHCO-), $8.04(1 \mathrm{H}, \mathrm{s},-\mathrm{CHN}-) .{ }^{13} \mathrm{C}-\mathrm{NMR}\left(500 \mathrm{MHz}, \mathrm{CDCl}_{3}\right): \delta 49.8,55.4,56.0,56.0,62.9,67.6,110.2,111.1,111.1$, $111.5,112.3,112.4,114.4,119.2,119.4,122.5,122.6,123.0,124.7,127.7,127.9,130.1,132.1,132.8,143.7$, 144.0, 144.5, 149.2, 149.5, 149.7, 151.2, 151.4, 151.6, 161.5, 188.6. LCMS (ES+) $m / z=706.2\left([\mathrm{M}+\mathrm{H}]^{+}\right.$, $t_{\mathrm{R}}=1.65 \mathrm{~min}$ ). HRMS (ESI+) $\mathrm{m} / z=728.2576[\mathrm{M}+\mathrm{Na}]^{+}$found, $\mathrm{C}_{40} \mathrm{H}_{39} \mathrm{O}_{9} \mathrm{~N}_{3} \mathrm{Na}^{+}$required 728.2579.

(E)-1-(3-Methoxy-4-((1-(2-(2-methoxy-4-((E)-3-(2,4,6-trimethoxyphenyl)acryloyl)phenoxy)ethyl)-1H-1,2,3triazol-4-yl)methoxy)phenyl)-3-(4-methoxyphenyl)prop-2-en-1-one (91). A mixture of alkyne chalcone 20 (234 mg, $0.727 \mathrm{mmol})$, azide chalcone 69 (303 mg, $0.732 \mathrm{mmol}), \mathrm{CuSO}_{4} \cdot 5 \mathrm{H}_{2} \mathrm{O}(203 \mathrm{mg}, 0.813 \mathrm{mmol})$ and sodium ascorbate $(361 \mathrm{mg}, 1.82 \mathrm{mmol})$ in $t-\mathrm{BuOH} / \mathrm{H}_{2} \mathrm{O}(1: 1,40 \mathrm{~mL})$ was reacted according to GP-A. The crude residue was purified by flash column chromatography $\left(\mathrm{SiO}_{2}, 1 \%-5 \% \mathrm{MeOH} / \mathrm{CH}_{2} \mathrm{Cl}_{2}\right)$ and recrystallized from $\mathrm{MeOH}$ to afford triazole hybrid $91(250 \mathrm{mg}, 47 \%)$ as a pale yellow-white flaky solid. m.p. 206-208 ${ }^{\circ} \mathrm{C}$. TLC $R_{\mathrm{f}}=0.44\left(5 \% \mathrm{MeOH} / \mathrm{CH}_{2} \mathrm{Cl}_{2}\right)$. IR $v_{\max }$ (neat) $/ \mathrm{cm}^{-1}: 3150 \mathrm{w}(\mathrm{C}-\mathrm{H} \mathrm{str}), 2942 \mathrm{w}$ (C-H str), 1661m (C=O str), 1601s ( $\mathrm{C}=\mathrm{C}$ str), 1572s (C=C str), 1514s (C=C str), 1459m, 1416m, 1324s, $1295 \mathrm{~m}, 1262 \mathrm{~s}, 1228 \mathrm{~m}, 1166 \mathrm{~s}, 1150 \mathrm{~s}, 1126 \mathrm{~s}, 1053 \mathrm{w}, 1015 \mathrm{~s} .{ }^{1} \mathrm{H}-\mathrm{NMR}\left(500 \mathrm{MHz}, \mathrm{CDCl}_{3}\right): \delta 3.86(3 \mathrm{H}, \mathrm{s}$, $\left.-\mathrm{OCH}_{3}\right), 3.87\left(3 \mathrm{H}, \mathrm{s},-\mathrm{OCH}_{3}\right), 3.91\left(6 \mathrm{H}, \mathrm{s}, 2 \times-\mathrm{OCH}_{3}\right), 3.92\left(3 \mathrm{H}, \mathrm{s},-\mathrm{OCH}_{3}\right), 3.95\left(3 \mathrm{H}, \mathrm{s},-\mathrm{OCH}_{3}\right), 4.46$ $\left(2 \mathrm{H}, \mathrm{t}, J=5.2 \mathrm{~Hz},-\mathrm{OCH}_{2} \mathrm{CH}_{2} \mathrm{~N}-\right), 4.84\left(2 \mathrm{H}, \mathrm{t}, J=5.2 \mathrm{~Hz},-\mathrm{OCH}_{2} \mathrm{CH}_{2} \mathrm{~N}-\right), 5.40\left(2 \mathrm{H}, \mathrm{s},-\mathrm{OCH}_{2} \mathrm{CN}-\right), 6.15$ $(2 \mathrm{H}, \mathrm{s}, \mathrm{ArH}), 6.85(1 \mathrm{H}, \mathrm{d}, J=8.4 \mathrm{~Hz}, \mathrm{ArH}), 6.94(2 \mathrm{H}, \mathrm{d}, J=8.8 \mathrm{~Hz}, \mathrm{ArH}), 7.17(1 \mathrm{H}, \mathrm{d}, J=8.8 \mathrm{~Hz}, \mathrm{ArH})$, $7.42(1 \mathrm{H}, \mathrm{d}, J=15.6 \mathrm{~Hz},-\mathrm{CH}=\mathrm{CHCO}-), 7.58-7.65(6 \mathrm{H}, \mathrm{m}, \mathrm{ArH}), 7.78(1 \mathrm{H}, \mathrm{d}, J=15.2 \mathrm{~Hz},-\mathrm{CH}=\mathrm{CHCO}-)$, $7.85(1 \mathrm{H}, \mathrm{d}, J=16.0 \mathrm{~Hz},-\mathrm{CH}=\mathrm{CHCO}-), 8.05(1 \mathrm{H}, \mathrm{s},-\mathrm{CHN}-), 8.23(1 \mathrm{H}, \mathrm{d}, J=16.0 \mathrm{~Hz},-\mathrm{CH}=\mathrm{CHCO}-)$. ${ }^{13} \mathrm{C}-\mathrm{NMR}\left(500 \mathrm{MHz}, \mathrm{CDCl}_{3}\right): \delta 49.8,55.4,55.8,55.9,56.0,62.9,67.5,90.5,106.6,111.1,111.7,112.3,112.4$, 114.4, 119.3, 121.6, 122.3, 122.6, 124.7, 127.8, 130.1, 132.1, 133.7, 135.6, 143.7, 143.9, 149.5, 149.5, 150.6, 151.6, 161.5, 161.7, 163.0, 188.6, 190.3. LCMS (ES+) $m / z=736.2\left([\mathrm{M}+\mathrm{H}]^{+}, t_{\mathrm{R}}=1.67 \mathrm{~min}\right)$. HRMS (ESI+) $m / z=736.2892[\mathrm{M}+\mathrm{H}]^{+}$found, $\mathrm{C}_{41} \mathrm{H}_{42} \mathrm{O}_{10} \mathrm{~N}_{3}{ }^{+}$required 736.2865. 
(E)-1-(2-Hydroxy-4-methoxyphenyl)-3-(4-methoxy-3-((1-(2-(2-methoxy-4-((E)-3-(2,4,6-trimethoxyphenyl)acryl oyl)phenoxy)ethyl)-1H-1,2,3- triazol-4-yl)methoxy)phenyl)prop-2-en-1-one (92). A mixture of alkyne chalcone 4 (167 mg, $0.494 \mathrm{mmol})$, azide chalcone 69 (206 mg, $0.498 \mathrm{mmol}), \mathrm{CuSO}_{4} \cdot 5 \mathrm{H}_{2} \mathrm{O}(140 \mathrm{mg}$, $0.560 \mathrm{mmol})$ and sodium ascorbate $(253 \mathrm{mg}, 1.28 \mathrm{mmol})$ in $t-\mathrm{BuOH} / \mathrm{H}_{2} \mathrm{O}(1: 1,40 \mathrm{~mL})$ was reacted according to GP-A. The crude residue was purified by flash column chromatography $\left(\mathrm{SiO}_{2}, 1 \%-5 \%\right.$ $\left.\mathrm{MeOH} / \mathrm{CH}_{2} \mathrm{Cl}_{2}\right)$ and recrystallized from MeOH to afford triazole hybrid $92(236 \mathrm{mg}, 64 \%)$ as a bright yellow flaky solid. m.p. $208-210^{\circ} \mathrm{C}$. TLC $R_{\mathrm{f}}=0.48\left(5 \% \mathrm{MeOH} / \mathrm{CH}_{2} \mathrm{Cl}_{2}\right)$. IR $v_{\max }$ (neat) $/ \mathrm{cm}^{-1}: 2931 \mathrm{w}$ (C-H str), 2842w (C-H str), 1632m (C=O str), 1597m (C=C str), 1568s (C=C str), 1509s (C=C str), 1455w, 1419w, 1371m, 1318m, 1259s, 1232s, 1211s, 1153s, 1123s, 1021m, 1004m. ${ }^{1} \mathrm{H}-\mathrm{NMR}\left(500 \mathrm{MHz}, \mathrm{CDCl}_{3}\right)$ : $\delta 3.86\left(3 \mathrm{H}, \mathrm{s},-\mathrm{OCH}_{3}\right), 3.87\left(3 \mathrm{H}, \mathrm{s},-\mathrm{OCH}_{3}\right), 3.91\left(3 \mathrm{H}, \mathrm{s},-\mathrm{OCH}_{3}\right), 3.91\left(9 \mathrm{H}, \mathrm{s}, 3 \times-\mathrm{OCH}_{3}\right), 4.45(2 \mathrm{H}, \mathrm{t}$, $\left.J=4.8 \mathrm{~Hz},-\mathrm{OCH}_{2} \mathrm{CH}_{2} \mathrm{~N}-\right), 4.84\left(2 \mathrm{H}, \mathrm{t}, J=4.8 \mathrm{~Hz},-\mathrm{OCH}_{2} \mathrm{CH}_{2} \mathrm{~N}-\right), 5.38\left(2 \mathrm{H}, \mathrm{s},-\mathrm{OCH} \mathrm{CN}^{-}\right), 6.14(2 \mathrm{H}, \mathrm{s}$, $\mathrm{ArH}), 6.46(1 \mathrm{H}, \mathrm{d}, J=2.4 \mathrm{~Hz}, \mathrm{ArH}), 6.50(1 \mathrm{H}, \mathrm{dd}, J=8.8,2.4 \mathrm{~Hz}, \mathrm{ArH}), 6.81(1 \mathrm{H}, \mathrm{d}, J=8.4 \mathrm{~Hz}, \mathrm{ArH})$, $6.89(1 \mathrm{H}, \mathrm{d}, J=2.4 \mathrm{~Hz}, \mathrm{ArH}), 7.21(1 \mathrm{H}, \mathrm{dd}, J=8.0,2.0 \mathrm{~Hz}, \mathrm{ArH}), 7.45(1 \mathrm{H}, \mathrm{d}, J=15.2 \mathrm{~Hz},-\mathrm{CH}=\mathrm{CHCO}-)$, $7.46(1 \mathrm{H}, \mathrm{d}, J=1.6 \mathrm{~Hz}, \mathrm{ArH}), 7.55(1 \mathrm{H}, \mathrm{dd}, J=8.4,1.6 \mathrm{~Hz}, \mathrm{ArH}), 7.61(1 \mathrm{H}, \mathrm{d}, J=1.6 \mathrm{~Hz}, \mathrm{ArH}), 7.77(1 \mathrm{H}$, $\mathrm{d}, J=15.6 \mathrm{~Hz},-\mathrm{CH}=\mathrm{CHCO}-), 7.85(1 \mathrm{H}, \mathrm{d}, J=16.0 \mathrm{~Hz},-\mathrm{CH}=\mathrm{CHCO}-), 7.89(1 \mathrm{H}, \mathrm{d}, J=8.8 \mathrm{~Hz}, \mathrm{ArH})$, $8.05(1 \mathrm{H}, \mathrm{s},-\mathrm{CHN}-), 8.23(1 \mathrm{H}, \mathrm{d}, J=16.0 \mathrm{~Hz},-\mathrm{CH}=\mathrm{CHCO}-), 13.55(1 \mathrm{H}, \mathrm{s}, \mathrm{OH}) .{ }^{13} \mathrm{C}-\mathrm{NMR}(500 \mathrm{MHz}$, $\left.\mathrm{CDCl}_{3}\right): \delta 49.8,55.4,55.5,55.8,55.9,56.0,63.2,67.6,90.5,101.0,106.6,107.6,111.5,111.6,112.3,113.0$, 114.2, 118.4, 121.5, 122.3, 124.3, 124.7, 127.8, 131.3, 133.7, 135.6, 144.0, 144.1, 147.8, 149.4, 150.6, 152.0, 161.7, 163.0, 166.0, 166.6, 190.3, 191.8. LCMS (ES+) $m / z=752.2\left([\mathrm{M}+\mathrm{H}]^{+}, t_{\mathrm{R}}=1.74 \mathrm{~min}\right)$. HRMS (ESI+) $m / z=752.2822[\mathrm{M}+\mathrm{H}]^{+}$found, $\mathrm{C}_{41} \mathrm{H}_{42} \mathrm{O}_{11} \mathrm{~N}_{3}{ }^{+}$required 752.2814.

(E)-1-(3-Methoxy-4-((1-(2-(2-methoxy-4-((E)-3-(2,3,4-trimethoxyphenyl)acryloyl)phenoxy)ethyl)-1H-1,2,3-tria zol-4-yl)methoxy)phenyl)-3-(4-methoxyphenyl)prop-2-en-1-one (93). A mixture of alkyne chalcone 20 (235 mg, $0.729 \mathrm{mmol}$ ), azide chalcone 68 (303 mg, $0.733 \mathrm{mmol}), \mathrm{CuSO}_{4} \cdot 5 \mathrm{H}_{2} \mathrm{O}(271 \mathrm{mg}, 1.09 \mathrm{mmol})$ and sodium ascorbate $(414 \mathrm{mg}, 2.09 \mathrm{mmol})$ in $t-\mathrm{BuOH} / \mathrm{H}_{2} \mathrm{O}(1: 1,40 \mathrm{~mL})$ was reacted according to GP-A. The crude residue was purified by flash column chromatography $\left(\mathrm{SiO}_{2}, 1 \%-5 \% \mathrm{MeOH} / \mathrm{CH}_{2} \mathrm{Cl}_{2}\right)$ and recrystallized from $\mathrm{MeOH}$ to afford triazole hybrid $93(515 \mathrm{mg}, 96 \%)$ as a pale yellow-green flaky solid. m.p. $128-130{ }^{\circ} \mathrm{C}$. TLC $R_{\mathrm{f}}=0.30\left(4 \% \mathrm{MeOH} / \mathrm{CH}_{2} \mathrm{Cl}_{2}\right)$. IR $v_{\max }$ (neat) $/ \mathrm{cm}^{-1}$ : 2937w (C-H str), 2836w (C-H str), 1652m (C=O str), 1595s (C=C str), 1572s (C=C str), 1510s (C=C str), 1494s, 1463s, 1415s, 1326w, 1255s, 1193w, 1148s, 1095s, 1021s. ${ }^{1} \mathrm{H}-\mathrm{NMR}\left(500 \mathrm{MHz}, \mathrm{CDCl}_{3}\right): \delta 3.86\left(3 \mathrm{H}, \mathrm{s},-\mathrm{OCH}_{3}\right), 3.90$ $\left(3 \mathrm{H}, \mathrm{s},-\mathrm{OCH}_{3}\right), 3.92\left(6 \mathrm{H}, \mathrm{s}, 2 \times-\mathrm{OCH}_{3}\right), 3.95\left(6 \mathrm{H}, \mathrm{s}, 2 \times-\mathrm{OCH}_{3}\right), 4.47\left(2 \mathrm{H}, \mathrm{t}, J=4.8 \mathrm{~Hz},-\mathrm{OCH}_{2} \mathrm{CH}_{2} \mathrm{~N}-\right)$, $4.84\left(2 \mathrm{H}, \mathrm{t}, J=4.8 \mathrm{~Hz},-\mathrm{OCH}_{2} \mathrm{CH}_{2} \mathrm{~N}-\right), 5.40\left(2 \mathrm{H}, \mathrm{s},-\mathrm{OCH}_{2} \mathrm{CN}-\right), 6.73(1 \mathrm{H}, \mathrm{d}, J=8.8 \mathrm{~Hz}, \mathrm{ArH}), 6.84(1 \mathrm{H}$, $\mathrm{d}, J=8.4 \mathrm{~Hz}, \mathrm{ArH}), 6.94(2 \mathrm{H}, \mathrm{d}, J=8.8 \mathrm{~Hz}, \mathrm{ArH}), 7.17(1 \mathrm{H}, \mathrm{d}, J=8.8 \mathrm{~Hz}, \mathrm{ArH}), 7.39(1 \mathrm{H}, \mathrm{d}, J=8.8$ $\mathrm{Hz}, \mathrm{ArH}), 7.42(1 \mathrm{H}, \mathrm{d}, J=15.6 \mathrm{~Hz},-\mathrm{CH}=\mathrm{CHCO}-), 7.54(1 \mathrm{H}, \mathrm{d}, J=16.0 \mathrm{~Hz},-\mathrm{CH}=\mathrm{CHCO}-), 7.59-7.64$ $(6 \mathrm{H}, \mathrm{m}, \mathrm{ArH}), 7.78(1 \mathrm{H}, \mathrm{d}, J=15.6 \mathrm{~Hz},-\mathrm{CH}=\mathrm{CHCO}-), 7.98(1 \mathrm{H}, \mathrm{d}, J=16.0 \mathrm{~Hz},-\mathrm{CH}=\mathrm{CHCO}-), 8.05$ (1H, s, -CHN-). ${ }^{13} \mathrm{C}-\mathrm{NMR}\left(500 \mathrm{MHz}, \mathrm{CDCl}_{3}\right): \delta$ 49.7, 55.4, 56.0, 56.0, 56.1, 60.9, 61.4, 62.9, 67.5, 107.6, $111.1,111.6,112.3,112.4,114.4,119.2,120.8,122.0,122.5,122.6,123.9,124.7,127.7,130.1,132.1,133.0$, 139.7, 142.5, 143.7, 144.0, 149.5, 149.7, 151.1, 151.6, 153.8, 155.7, 161.5, 188.6, 189.0. LCMS (ES+) $m / z$ $=736.2\left([\mathrm{M}+\mathrm{H}]^{+}, t_{\mathrm{R}}=1.66 \mathrm{~min}\right)$. HRMS (ESI+) $m / z=736.2855[\mathrm{M}+\mathrm{H}]^{+}$found, $\mathrm{C}_{41} \mathrm{H}_{42} \mathrm{O}_{10} \mathrm{~N}_{3}{ }^{+}$ required 736.2870 .

(E)-1-(3-Methoxy-4-((1-(2-(2-methoxy-4-((E)-3-(2,4,6-trimethoxyphenyl)acryloyl)phenoxy)ethyl)-1H-1,2,3-tria zol-4-yl)methoxy)phenyl)-3-(1-methyl-1H-pyrrol-2-yl)prop-2-en-1-one (94). A mixture of alkyne chalcone 22 (217 mg, $0.734 \mathrm{mmol})$, azide chalcone 69 (305 mg, $0.737 \mathrm{mmol}), \mathrm{CuSO}_{4} \cdot 5 \mathrm{H}_{2} \mathrm{O}(214 \mathrm{mg}, 0.857 \mathrm{mmol})$ and sodium ascorbate $(365 \mathrm{mg}, 1.84 \mathrm{mmol})$ in $t-\mathrm{BuOH} / \mathrm{H}_{2} \mathrm{O}(1: 1,40 \mathrm{~mL})$ was reacted according to GP-A. The crude residue was purified by flash column chromatography $\left(\mathrm{SiO}_{2}, 1 \%-5 \% \mathrm{MeOH} / \mathrm{CH}_{2} \mathrm{Cl}_{2}\right)$ and recrystallized from $\mathrm{MeOH}$ to afford triazole hybrid $94(367 \mathrm{mg}, 70 \%)$ as a bright yellow flaky solid. m.p. $138-140{ }^{\circ} \mathrm{C}$. TLC $R_{\mathrm{f}}=0.31\left(5 \% \mathrm{MeOH} / \mathrm{CH}_{2} \mathrm{Cl}_{2}\right)$. IR $v_{\max }$ (neat) $/ \mathrm{cm}^{-1}$ : 2941w (C-H str), 2838w (C-H str), 1646m (C=O str), 1596m (C=C str), 1566s (C=C str), 1512m (C=C str), 1470m, 1457m, 1414m, $1338 \mathrm{w}, 1321 \mathrm{~m}, 1257 \mathrm{~s}, 1198 \mathrm{~m}, 1155 \mathrm{~s}, 1146 \mathrm{~s}, 1118 \mathrm{~s}, 1027 \mathrm{~m} .{ }^{1} \mathrm{H}-\mathrm{NMR}\left(500 \mathrm{MHz}, \mathrm{CDCl}_{3}\right): \delta 3.77(3 \mathrm{H}, \mathrm{s}$, $\left.-\mathrm{NCH}_{3}\right), 3.86\left(3 \mathrm{H}, \mathrm{s},-\mathrm{OCH}_{3}\right), 3.91\left(6 \mathrm{H}, \mathrm{s}, 2 \times-\mathrm{OCH}_{3}\right), 3.91\left(3 \mathrm{H}, \mathrm{s},-\mathrm{OCH}_{3}\right), 3.94\left(3 \mathrm{H}, \mathrm{s},-\mathrm{OCH}_{3}\right), 4.45$ 
$\left(2 \mathrm{H}, \mathrm{t}, J=4.8 \mathrm{~Hz},-\mathrm{OCH}_{2} \mathrm{CH}_{2} \mathrm{~N}-\right), 4.83\left(2 \mathrm{H}, \mathrm{t}, J=4.8 \mathrm{~Hz},-\mathrm{OCH}_{2} \mathrm{CH}_{2} \mathrm{~N}-\right), 5.38\left(2 \mathrm{H}, \mathrm{s},-\mathrm{OCH}_{2} \mathrm{CN}-\right), 6.14$ $(2 \mathrm{H}, \mathrm{s}, \mathrm{ArH}), 6.21-6.23(1 \mathrm{H}, \mathrm{m}, \mathrm{ArH}), 6.80-6.86(3 \mathrm{H}, \mathrm{m}, \mathrm{ArH}), 7.16(1 \mathrm{H}, \mathrm{d}, J=8.8 \mathrm{~Hz}, \mathrm{ArH}), 7.29(1 \mathrm{H}, \mathrm{d}$, $J=15.6 \mathrm{~Hz},-\mathrm{CH}=\mathrm{CHCO}-), 7.57-7.63(4 \mathrm{H}, \mathrm{m}, \mathrm{ArH}), 7.79(1 \mathrm{H}, \mathrm{d}, J=15.2 \mathrm{~Hz},-\mathrm{CH}=\mathrm{CHCO}-), 7.85(1 \mathrm{H}$, $\mathrm{d}, J=16.0 \mathrm{~Hz},-\mathrm{CH}=\mathrm{CHCO}-), 8.05(1 \mathrm{H}, \mathrm{s},-\mathrm{CHN}-), 8.23(1 \mathrm{H}, \mathrm{d}, J=15.6 \mathrm{~Hz},-\mathrm{CH}=\mathrm{CHCO}-) .{ }^{13} \mathrm{C}-\mathrm{NMR}$ (500 MHz, $\mathrm{CDCl}_{3}$ ): $\delta 34.4,49.7,55.4,55.8,55.9,56.0,62.8,67.5,90.5,106.5,109.6,111.1,111.7,112.1$, $112.3,112.4,116.3,121.5,122.3,122.3,124.7,127.6,130.3,131.6,132.4,133.7,135.6,143.7,149.4,149.5$, 150.6, 151.4, 161.6, 163.0, 188.1, 190.3. LCMS (ES+) $m / z=709.3\left([\mathrm{M}+\mathrm{H}]^{+}, t_{\mathrm{R}}=1.63 \mathrm{~min}\right)$. HRMS (ESI+) $m / z=709.2854[\mathrm{M}+\mathrm{H}]^{+}$found, $\mathrm{C}_{39} \mathrm{H}_{41} \mathrm{O}_{9} \mathrm{~N}_{4}{ }^{+}$required 709.2868.

(E)-1-(3-Methoxy-4-((1-(2-(2-methoxy-4-((E)-3-(1-methyl-1H-pyrrol-2-yl)acryloyl)phenoxy)ethyl)-1H-1,2,3-tria zol-4-yl)methoxy)phenyl)-3-(1-methyl-1H-pyrrol-2-yl)prop-2-en-1-one (95). A mixture of alkyne chalcone 22 ( $271 \mathrm{mg}, 0.918 \mathrm{mmol}$ ), azide chalcone 70 (301 mg, $0.924 \mathrm{mmol}), \mathrm{CuSO}_{4} \cdot 5 \mathrm{H}_{2} \mathrm{O}(316 \mathrm{~g}, 1.26 \mathrm{mmol}$ ) and sodium ascorbate $(499 \mathrm{mg}, 2.52 \mathrm{mmol})$ in $t-\mathrm{BuOH} / \mathrm{H}_{2} \mathrm{O}(1: 1,40 \mathrm{~mL})$ was reacted according to GP-A. The crude residue was purified by flash column chromatography $\left(\mathrm{SiO}_{2}, 1 \%-5 \% \mathrm{MeOH} / \mathrm{CH}_{2} \mathrm{Cl}_{2}\right)$ and recrystallized from $\mathrm{MeOH}$ to afford triazole hybrid 95 (555 $\mathrm{mg}, 97 \%)$ as a dark yellow-brown flaky solid. m.p. $118-120^{\circ} \mathrm{C}$. TLC $R_{\mathrm{f}}=0.38\left(5 \% \mathrm{MeOH} / \mathrm{CH}_{2} \mathrm{Cl}_{2}\right)$. IR $\gamma_{\max }$ (neat) $/ \mathrm{cm}^{-1}: 2941 \mathrm{w}(\mathrm{C}-\mathrm{H}$ str), 2844w (C-H str), 1644m (C=O str), 1594m (C=C str), 1564s (C=C str), 1511m (C=C str), 1480m, 1412m, 1381w, 1330m, 1258s, 1196m, 1153s, 1129m, 1055w, 1025m. ${ }^{1} \mathrm{H}-\mathrm{NMR}\left(500 \mathrm{MHz}, \mathrm{CDCl}_{3}\right): \delta 3.76$ $\left(3 \mathrm{H}, \mathrm{s},-\mathrm{NCH}_{3}\right), 3.77\left(3 \mathrm{H}, \mathrm{s},-\mathrm{NCH}_{3}\right), 3.92\left(3 \mathrm{H}, \mathrm{s},-\mathrm{OCH}_{3}\right), 3.95\left(3 \mathrm{H}, \mathrm{s},-\mathrm{OCH}_{3}\right), 4.46(2 \mathrm{H}, \mathrm{t}, J=4.8 \mathrm{~Hz}$, $\left.-\mathrm{OCH}_{2} \mathrm{CH}_{2} \mathrm{~N}-\right), 4.84\left(2 \mathrm{H}, \mathrm{t}, J=4.8 \mathrm{~Hz},-\mathrm{OCH}_{2} \mathrm{CH}_{2} \mathrm{~N}-\right), 5.39\left(2 \mathrm{H}, \mathrm{s},-\mathrm{OCH}_{2} \mathrm{CN}-\right), 6.22-6.24(2 \mathrm{H}, \mathrm{m}$, $\mathrm{ArH}), 6.82-6.86(5 \mathrm{H}, \mathrm{m}, \mathrm{ArH}), 7.15(1 \mathrm{H}, \mathrm{d}, J=8.0 \mathrm{~Hz}, \mathrm{ArH}), 7.27(1 \mathrm{H}, \mathrm{d}, J=15.2 \mathrm{~Hz},-\mathrm{CH}=\mathrm{CHCO}-$, overlain by $\left.\mathrm{CDCl}_{3}\right), 7.29(1 \mathrm{H}, \mathrm{d}, J=14.0 \mathrm{~Hz},-\mathrm{CH}=\mathrm{CHCO}-), 7.57-7.62(4 \mathrm{H}, \mathrm{m}, \mathrm{ArH}), 7.79(2 \mathrm{H}, \mathrm{d}$, $J=14.8 \mathrm{~Hz}, 2 \times-\mathrm{CH}=\mathrm{CHCO}-), 8.04(1 \mathrm{H}, \mathrm{s},-\mathrm{CHN}-) .{ }^{13} \mathrm{C}-\mathrm{NMR}\left(500 \mathrm{MHz}, \mathrm{CDCl}_{3}\right): \delta 34.4,49.7,55.9$, 56.0, 62.9, 67.5, 109.6, 109.7, 111.1, 111.4, 112.1, 112.2, 112.3, 112.4, 116.2, 116.3, 122.1, 122.3, 124.7, 127.6, 127.7, 130.3, 130.3, 131.6, 131.8, 132.4, 133.1, 143.7, 149.4, 149.6, 150.9, 151.4, 188.0, 188.1. LCMS (ES+) $m / z=622.3\left([\mathrm{M}+\mathrm{H}]^{+}, t_{\mathrm{R}}=1.62 \mathrm{~min}\right)$. HRMS (ESI +$) m / z=622.2648[\mathrm{M}+\mathrm{H}]^{+}$found, $\mathrm{C}_{35} \mathrm{H}_{36} \mathrm{O}_{6} \mathrm{~N}_{5}{ }^{+}$ required 622.2660 .

(E)-1-(2-Hydroxyphenyl)-3-(4-(2-(4-((2-((E)-3-(1-methyl-1H-pyrrol-2-yl)acryloyl)phenoxy)methyl)-1H-1,2,3triazol-1-yl)ethoxy)phenyl)prop-2-en-1-one (96). A mixture of alkyne chalcone 23 (337 mg, $1.27 \mathrm{mmol}$ ), azide chalcone $61(400 \mathrm{mg}, 1.29 \mathrm{mmol}), \mathrm{CuSO}_{4} \cdot 5 \mathrm{H}_{2} \mathrm{O}(429 \mathrm{mg}, 1.72 \mathrm{mmol})$ and sodium ascorbate (641 mg, $3.23 \mathrm{mmol})$ in $t-\mathrm{BuOH} / \mathrm{H}_{2} \mathrm{O}(1: 1,40 \mathrm{~mL})$ was reacted according to GP-A. The crude residue was purified by flash column chromatography $\left(\mathrm{SiO}_{2}, 1 \%-5 \% \mathrm{MeOH} / \mathrm{CH}_{2} \mathrm{Cl}_{2}\right)$ to afford triazole hybrid 96 (399 mg, 55\%) as a bright yellow-orange powdery solid. m.p. $98-100{ }^{\circ} \mathrm{C}$. TLC $R_{\mathrm{f}}=0.40(3 \%$ $\mathrm{MeOH} / \mathrm{CH}_{2} \mathrm{Cl}_{2}$ ). IR $v_{\max }$ (neat) $/ \mathrm{cm}^{-1}$ : 2933w (C-H str), 2884w (C-H str), $1635 \mathrm{~m}$ (C=O str), 1597m (C=C str), 1562s (C=C str), 1509m (C=C str), 1480m, 1447m, 1329m, 1286m, 1268m, 1233m, 1202s, 1174s, 1156s, 1113w, 1055m, 1025s. ${ }^{1} \mathrm{H}-\mathrm{NMR}\left(500 \mathrm{MHz}, \mathrm{CDCl}_{3}\right): \delta 3.65\left(3 \mathrm{H}, \mathrm{s},-\mathrm{NCH}_{3}\right), 4.32(2 \mathrm{H}, \mathrm{t}, J=5.2 \mathrm{~Hz}$, $\left.-\mathrm{OCH}_{2} \mathrm{CH}_{2} \mathrm{~N}-\right), 4.65\left(2 \mathrm{H}, \mathrm{t}, J=5.2 \mathrm{~Hz},-\mathrm{OCH}_{2} \mathrm{CH}_{2} \mathrm{~N}-\right), 5.33\left(2 \mathrm{H}, \mathrm{s},-\mathrm{OCH}_{2} \mathrm{CN}-\right), 6.16-6.18(1 \mathrm{H}, \mathrm{m}, \mathrm{ArH})$, $6.63(1 \mathrm{H}, \mathrm{dd}, J=4.0,1.2 \mathrm{~Hz}, \mathrm{ArH}), 6.76(1 \mathrm{H}, \mathrm{t}, J=2.0 \mathrm{~Hz}, \mathrm{ArH}), 6.85(2 \mathrm{H}, \mathrm{d}, J=8.8 \mathrm{~Hz}, \mathrm{ArH}), 6.95$ $(1 \mathrm{H}, \mathrm{t}, J=8.0 \mathrm{~Hz}, \mathrm{ArH}), 7.03(1 \mathrm{H}, \mathrm{dd}, J=8.4,0.8 \mathrm{~Hz}, \operatorname{ArH}), 7.08(1 \mathrm{H}, \mathrm{d}, J=7.6 \mathrm{~Hz}, \operatorname{ArH}), 7.12(1 \mathrm{H}, \mathrm{d}$, $J=8.4 \mathrm{~Hz}, \mathrm{ArH}), 7.16(1 \mathrm{H}, \mathrm{d}, J=15.6 \mathrm{~Hz},-\mathrm{CH}=\mathrm{CHCO}-), 7.44-7.59(4 \mathrm{H}, \mathrm{m}, \mathrm{ArH}), 7.54(1 \mathrm{H}, \mathrm{d}, J=15.6$ $\mathrm{Hz},-\mathrm{CH}=\mathrm{CHCO}-), 7.60(1 \mathrm{H}, \mathrm{d}, J=15.2 \mathrm{~Hz},-\mathrm{CH}=\mathrm{CHCO}-), 7.66(1 \mathrm{H}, \mathrm{dd}, J=8.0,2.0 \mathrm{~Hz}, \mathrm{ArH}), 7.76(1 \mathrm{H}$, s, $-\mathrm{CHN}-), 7.86(1 \mathrm{H}, \mathrm{d}, J=15.6 \mathrm{~Hz},-\mathrm{CH}=\mathrm{CHCO}-), 7.93(1 \mathrm{H}, \mathrm{dd}, J=8.4,1.6 \mathrm{~Hz}, \mathrm{ArH}), 12.89(1 \mathrm{H}, \mathrm{s}, \mathrm{OH})$. ${ }^{13} \mathrm{C}-\mathrm{NMR}\left(500 \mathrm{MHz}, \mathrm{CDCl}_{3}\right)$ : $\delta 34.3,49.5,63.0,66.2,109.6,112.4,113.0,114.9,118.2,118.5,118.8,120.0$, 121.4, 122.2, 123.9, 127.7, 128.2, 129.5, 130.0, 130.1, 130.5, 130.6, 132.7, 136.2, 144.1, 144.8, 156.5, 159.9, 163.5, 191.9, 193.5. LCMS (ES+) $m / z=575.3\left([\mathrm{M}+\mathrm{H}]^{+}, t_{\mathrm{R}}=1.72 \mathrm{~min}\right)$. HRMS (ESI+) $m / z=575.2266$ $[\mathrm{M}+\mathrm{H}]^{+}$found, $\mathrm{C}_{34} \mathrm{H}_{31} \mathrm{O}_{5} \mathrm{~N}_{4}{ }^{+}$required 575.2289.

(E)-3-(Ferrocenyl)-1-(4-((1-(2-(4-((E)-3-(2-hydroxyphenyl)-3-oxoprop-1-en-1-yl)phenoxy)ethyl)-1H-1,2,3-triaz ol-4-yl)methoxy)-3-methoxyphenyl)prop-2-en-1-one (97). A mixture of alkyne chalcone 25 (301 mg, $0.753 \mathrm{mmol})$, azide chalcone $61(235 \mathrm{mg}, 0.760 \mathrm{mmol}), \mathrm{CuSO}_{4} \cdot 5 \mathrm{H}_{2} \mathrm{O}(225 \mathrm{mg}, 0.900 \mathrm{mmol})$ and sodium ascorbate $(398 \mathrm{mg}, 2.01 \mathrm{mmol})$ in $t-\mathrm{BuOH} / \mathrm{H}_{2} \mathrm{O}(1: 1,40 \mathrm{~mL})$ was reacted according to GP-A. The 
crude residue was purified by flash column chromatography $\left(\mathrm{SiO}_{2}, 1 \%-5 \% \mathrm{MeOH} / \mathrm{CH}_{2} \mathrm{Cl}_{2}\right)$ to afford triazole hybrid $97(427 \mathrm{mg}, 80 \%)$ as a dark red-brown powdery solid. m.p. $178-180{ }^{\circ} \mathrm{C}$. TLC $R_{\mathrm{f}}=0.28$ $\left(2 \% \mathrm{MeOH} / \mathrm{CH}_{2} \mathrm{Cl}_{2}\right)$. IR $v_{\max }$ (neat) $/ \mathrm{cm}^{-1}: 2937 \mathrm{w}$ (C-H str), 1740m (C=O str), 1651m, 1637s, 1596s (C=C str), 1571s (C=C str), 1510s (C=C str), 1487m, 1444m, 1341w, 1300m, 1266s, 1203s, 1179s, 1159s, 1146s, 1048m, 1020s. ${ }^{1} \mathrm{H}-\mathrm{NMR}\left(500 \mathrm{MHz}, \mathrm{CDCl}_{3}\right): \delta 3.98\left(3 \mathrm{H}, \mathrm{s},-\mathrm{OCH}_{3}\right), 4.16\left(5 \mathrm{H}, \mathrm{s},-\mathrm{C}_{5} \mathrm{H}_{5}\right), 4.42(2 \mathrm{H}, \mathrm{t}$, $\left.J=5.2 \mathrm{~Hz},-\mathrm{OCH}_{2} \mathrm{CH}_{2} \mathrm{~N}-\right), 4.47\left(2 \mathrm{H}, \mathrm{t}, J=2.0 \mathrm{~Hz},-\mathrm{C}_{5} \mathrm{H}_{4}\right), 4.56\left(2 \mathrm{H}, \mathrm{t}, J=2.0 \mathrm{~Hz},-\mathrm{C}_{5} \mathrm{H}_{4}\right), 4.80(2 \mathrm{H}, \mathrm{t}$, $\left.J=5.2 \mathrm{~Hz},-\mathrm{OCH}_{2} \mathrm{CH}_{2} \mathrm{~N}-\right), 5.43\left(2 \mathrm{H}, \mathrm{s},-\mathrm{OCH}_{2} \mathrm{CN}-\right), 6.84(2 \mathrm{H}, \mathrm{d}, J=8.8 \mathrm{~Hz}, \mathrm{ArH}), 6.97(1 \mathrm{H}, \mathrm{t}, J=8.0 \mathrm{~Hz}$, $\mathrm{ArH}), 7.04(1 \mathrm{H}, \mathrm{dd}, J=8.4,0.8 \mathrm{~Hz}, \mathrm{ArH}), 7.12(1 \mathrm{H}, \mathrm{d}, J=15.2 \mathrm{~Hz},-\mathrm{CH}=\mathrm{CHCO}-), 7.12(1 \mathrm{H}, \mathrm{d}, J=8.4 \mathrm{~Hz}$, $\mathrm{ArH}), 7.51(1 \mathrm{H}, \mathrm{t}, J=8.4 \mathrm{~Hz}, \mathrm{ArH}), 7.54-7.61(3 \mathrm{H}, \mathrm{m}, \mathrm{ArH}), 7.59(1 \mathrm{H}, \mathrm{d}, J=16.0 \mathrm{~Hz},-\mathrm{CH}=\mathrm{CHCO}-)$, $7.64(1 \mathrm{H}, \mathrm{d}, J=1.6 \mathrm{~Hz}, \mathrm{ArH}), 7.74(1 \mathrm{H}, \mathrm{d}, J=15.6 \mathrm{~Hz},-\mathrm{CH}=\mathrm{CHCO}-), 7.86(1 \mathrm{H}, \mathrm{s},-\mathrm{CHN}-), 7.86(1 \mathrm{H}, \mathrm{d}$, $J=15.6 \mathrm{~Hz},-\mathrm{CH}=\mathrm{CHCO}-), 7.97(1 \mathrm{H}, \mathrm{dd}, J=8.0,1.6 \mathrm{~Hz}, \mathrm{ArH}), 12.90(1 \mathrm{H}, \mathrm{s}, \mathrm{OH}) .{ }^{13} \mathrm{C}-\mathrm{NMR}(500 \mathrm{MHz}$, $\left.\mathrm{CDCl}_{3}\right): \delta 49.7,56.1,62.8,66.3,68.9,69.7,71.3,79.2,111.1,112.3,114.9,118.3,118.5,118.6,118.8,120.0$, 122.4, 124.3, 128.4, 129.6, 130.6, 132.3, 136.3, 143.8, 144.7, 146.1, 149.5, 151.2, 159.8, 163.6, 187.9, 193.6. LCMS (ES+) $m / z=710.2\left([\mathrm{M}+\mathrm{H}]^{+}, t_{\mathrm{R}}=2.09 \mathrm{~min}\right)$. HRMS $(\mathrm{ESI}+) m / z=710.1889[\mathrm{M}+\mathrm{H}]^{+}$found, $\mathrm{C}_{40} \mathrm{H}_{36} \mathrm{~N}_{3} \mathrm{O}_{6} \mathrm{Fe}^{+}$required 710.1875 .

(E)-3-((1-(2-(4-(3-(3,4-Dimethoxyphenyl)acryloyl)-2-methoxyphenoxy)ethyl)-1H-1,2,3-triazol-4-yl)methoxy)-2phenyl-4H-chromen-4-one (98). A mixture of alkyne flavone 30 (806 mg, $2.92 \mathrm{mmol}$ ), azide chalcone $67(1.01 \mathrm{~g}, 2.64 \mathrm{mmol}), \mathrm{CuSO}_{4} \cdot 5 \mathrm{H}_{2} \mathrm{O}(716 \mathrm{mg}, 2.87 \mathrm{mmol})$ and sodium ascorbate $(1.29 \mathrm{~g}, 6.51 \mathrm{mmol})$ in $t-\mathrm{BuOH} / \mathrm{H}_{2} \mathrm{O}(1: 1,40 \mathrm{~mL})$ was reacted according to GP-A. The crude residue was purified by flash column chromatography $\left(\mathrm{SiO}_{2}, 1 \%-5 \% \mathrm{MeOH} / \mathrm{CH}_{2} \mathrm{Cl}_{2}\right)$ to afford triazole hybrid $98(622 \mathrm{mg}$, $36 \%)$ as a pale yellow-green powdery solid. m.p. $102-104{ }^{\circ} \mathrm{C}$. TLC $R_{\mathrm{f}}=0.24\left(3 \% \mathrm{MeOH} / \mathrm{CH}_{2} \mathrm{Cl}_{2}\right)$. IR $v_{\max }$ (neat) $/ \mathrm{cm}^{-1}$ : 2940w (C-H str), 2836w (C-H str), 1644m (C=O str), 1596m (C=C str), 1579m $\left(\mathrm{C}=\mathrm{C}\right.$ str), 1510s (C=C str), 1467m, 1420w, 1398w, 1260s, 1237s, 1196m, 1146s, 1138s, 1022s. ${ }^{1} \mathrm{H}-\mathrm{NMR}$ $\left(500 \mathrm{MHz}_{\mathrm{CDCl}}\right): \delta 3.87\left(3 \mathrm{H}, \mathrm{s},-\mathrm{OCH}_{3}\right), 3.94\left(3 \mathrm{H}, \mathrm{s},-\mathrm{OCH}_{3}\right), 3.96\left(3 \mathrm{H}, \mathrm{s},-\mathrm{OCH}_{3}\right), 4.38(2 \mathrm{H}, \mathrm{t}$, $\left.J=5.2 \mathrm{~Hz},-\mathrm{OCH}_{2} \mathrm{CH}_{2} \mathrm{~N}-\right), 4.74\left(2 \mathrm{H}, \mathrm{t}, J=5.2 \mathrm{~Hz},-\mathrm{OCH}_{2} \mathrm{CH}_{2} \mathrm{~N}-\right), 5.32\left(2 \mathrm{H}, \mathrm{s},-\mathrm{OCH}_{2} \mathrm{CN}-\right), 6.84(1 \mathrm{H}$, $\mathrm{d}, J=8.4 \mathrm{~Hz}, \mathrm{ArH}), 6.90(1 \mathrm{H}, \mathrm{d}, J=8.4 \mathrm{~Hz}, \mathrm{ArH}), 7.16(1 \mathrm{H}, \mathrm{d}, J=1.6 \mathrm{~Hz}, \mathrm{ArH}), 7.24(1 \mathrm{H}, \mathrm{dd}, J=8.4$, $1.6 \mathrm{~Hz}, \mathrm{ArH}), 7.39(1 \mathrm{H}, \mathrm{d}, J=15.6 \mathrm{~Hz},-\mathrm{CH}=\mathrm{CHCO}-), 7.39-7.44(4 \mathrm{H}, \mathrm{m}, \mathrm{ArH}), 7.50(1 \mathrm{H}, \mathrm{d}, J=8.4 \mathrm{~Hz}$, $\mathrm{ArH}), 7.59(1 \mathrm{H}, \mathrm{d}, J=1.6 \mathrm{~Hz}, \mathrm{ArH}), 7.62(1 \mathrm{H}, \mathrm{dd}, J=8.4,2.0 \mathrm{~Hz}, \mathrm{ArH}), 7.69(1 \mathrm{H}, \mathrm{t}, J=8.4 \mathrm{~Hz}, \mathrm{ArH})$, $7.77(1 \mathrm{H}, \mathrm{d}, J=15.6 \mathrm{~Hz},-\mathrm{CH}=\mathrm{CHCO}-), 7.96(1 \mathrm{H}, \mathrm{s},-\mathrm{CHN}-), 7.97-8.00(2 \mathrm{H}, \mathrm{m}, \mathrm{ArH}), 8.27$ (1H, dd, $J=8.0,1.6 \mathrm{~Hz}, \mathrm{ArH}) .{ }^{13} \mathrm{C}-\mathrm{NMR}\left(500 \mathrm{MHz} \mathrm{CDCl}_{3}\right): \delta 49.4,56.0,56.0,65.1,67.4,110.2,111.1,111.5,112.2$, $118.1,119.5,122.4,123.0,124.1,124.8,125.3,125.7,127.9,128.3,128.7,130.6,130.7,132.7,133.5,139.4$, $143.9,144.5,149.2,149.7,151.3,151.3,155.3,156.4,175.0,188.6$. LCMS (ES+) $m / z=660.2\left([\mathrm{M}+\mathrm{H}]^{+}\right.$, $t_{\mathrm{R}}=1.64 \mathrm{~min}$ ). HRMS (ESI+) $m / z=660.2325[\mathrm{M}+\mathrm{H}]^{+}$found, $\mathrm{C}_{38} \mathrm{H}_{34} \mathrm{O}_{8} \mathrm{~N}_{3}{ }^{+}$required 660.2340.

(E)-3-((1-(2-(2-Methoxy-4-(3-(2,3,4-trimethoxyphenyl)acryloyl)phenoxy)ethyl)-1H-1,2,3-triazol-4-yl)methoxy)2-phenyl-4H-chromen-4-one (99). A mixture of alkyne flavone 30 (205 mg, $0.742 \mathrm{mmol}$ ), azide chalcone 68 (306 mg, $0.739 \mathrm{mmol}), \mathrm{CuSO}_{4} \cdot 5 \mathrm{H}_{2} \mathrm{O}(204 \mathrm{mg}, 0.818 \mathrm{mmol})$ and sodium ascorbate $(365 \mathrm{mg}, 1.84 \mathrm{mmol})$ in $t$ - $\mathrm{BuOH} / \mathrm{H}_{2} \mathrm{O}(1: 1,40 \mathrm{~mL})$ was reacted according to GP-A. The crude residue was purified by flash column chromatography $\left(\mathrm{SiO}_{2}, 1 \%-5 \% \mathrm{MeOH} / \mathrm{CH}_{2} \mathrm{Cl}_{2}\right)$ and recrystallized from $\mathrm{MeOH}$ to afford triazole hybrid 99 (178 mg, 35\%) as an off-white powdery solid. m.p. $120-122{ }^{\circ} \mathrm{C}$. TLC $R_{\mathrm{f}}=0.28(5 \%$ $\mathrm{MeOH} / \mathrm{CH}_{2} \mathrm{Cl}_{2}$ ). IR $v_{\max }$ (neat)/cm ${ }^{-1}$ : 2941w (C-H str), 2838w (C-H str), 1740w, 1645s (C=O str), 1581s (C=C str), 1563m (C=C str), 1516w (C=C str), 1494s, 1466s, 1414m, 1403m, 1264s, 1238m, 1195m, $1148 \mathrm{~m}, 1096 \mathrm{~s}, 1039 \mathrm{~m}, 1027 \mathrm{~m} .{ }^{1} \mathrm{H}-\mathrm{NMR}\left(500 \mathrm{MHz}, \mathrm{CDCl}_{3}\right): \delta 3.86\left(3 \mathrm{H}, \mathrm{s},-\mathrm{OCH}_{3}\right), 3.90\left(3 \mathrm{H}, \mathrm{s},-\mathrm{OCH}_{3}\right)$, $3.92\left(3 \mathrm{H}, \mathrm{s},-\mathrm{OCH}_{3}\right), 3.95\left(3 \mathrm{H}, \mathrm{s},-\mathrm{OCH}_{3}\right), 4.36\left(2 \mathrm{H}, \mathrm{t}, J=5.2 \mathrm{~Hz},-\mathrm{OCH}_{2} \mathrm{CH}_{2} \mathrm{~N}-\right), 4.73(2 \mathrm{H}, \mathrm{t}, J=5.2 \mathrm{~Hz}$, $\left.-\mathrm{OCH}_{2} \mathrm{CH}_{2} \mathrm{~N}-\right), 5.33\left(2 \mathrm{H}, \mathrm{s},-\mathrm{OCH}_{2} \mathrm{CN}-\right), 6.73(1 \mathrm{H}, \mathrm{d}, J=8.8 \mathrm{~Hz}, \mathrm{ArH}), 6.84(1 \mathrm{H}, \mathrm{d}, J=8.0 \mathrm{~Hz}, \mathrm{ArH})$, 7.38-7.44 (5H, m, ArH), 7.50 (1H, d, J = 8.4 Hz, ArH), 7.55 (1H, d, J = 15.6 Hz, -CH=CHCO-), 7.59-7.62 $(2 \mathrm{H}, \mathrm{m}, \mathrm{ArH}), 7.69(1 \mathrm{H}, \mathrm{t}, J=8.4 \mathrm{~Hz}, \mathrm{ArH}), 7.95(1 \mathrm{H}, \mathrm{s},-\mathrm{CHN}-), 7.96-7.99(2 \mathrm{H}, \mathrm{m}, \mathrm{ArH}), 7.99(1 \mathrm{H}, \mathrm{d}$, $J=15.6 \mathrm{~Hz},-\mathrm{CH}=\mathrm{CHCO}-), 8.28(1 \mathrm{H}, \mathrm{dd}, J=8.0,1.2 \mathrm{~Hz}, \mathrm{ArH}) .{ }^{13} \mathrm{C}-\mathrm{NMR}\left(500 \mathrm{MHz}, \mathrm{CDCl}_{3}\right): \delta 49.4$, 55.9, 56.1, 60.9, 61.4, 65.1, 67.4, 107.6, 111.5, 112.2, 118.1, 120.9, 122.0, 122.4, 123.9, 124.1, 124.8, 125.3, $125.7,128.3,128.7,130.6,130.7,132.8,133.5,139.4,139.7,142.5,143.9,149.6,151.2,153.7,155.3,155.7$, 
156.4, 175.0, 189.0. LCMS (ES+) $m / z=690.2\left([\mathrm{M}+\mathrm{H}]^{+}, t_{\mathrm{R}}=1.95 \mathrm{~min}\right)$. HRMS (ESI+) $m / z=690.2425$ $[\mathrm{M}+\mathrm{H}]^{+}$found, $\mathrm{C}_{39} \mathrm{H}_{36} \mathrm{O}_{9} \mathrm{~N}_{3}{ }^{+}$required 690.2446 .

(E)-3-((1-(2-(2-Methoxy-4-(3-(2,4,6-trimethoxyphenyl)acryloyl)phenoxy)ethyl)-1H-1,2,3-triazol-4-yl)methoxy)2-phenyl-4H-chromen-4-one (100). A mixture of alkyne flavone 30 (207 mg, $0.749 \mathrm{mmol})$, azide chalcone 69 (306 mg, $0.741 \mathrm{mmol}), \mathrm{CuSO}_{4} \cdot 5 \mathrm{H}_{2} \mathrm{O}(203 \mathrm{mg}, 0.814 \mathrm{mmol})$ and sodium ascorbate $(369 \mathrm{mg}$, $1.86 \mathrm{mmol})$ in $t-\mathrm{BuOH} / \mathrm{H}_{2} \mathrm{O}(1: 1,40 \mathrm{~mL})$ was reacted according to GP-A. The crude residue was purified by flash column chromatography $\left(\mathrm{SiO}_{2}, 1 \%-5 \% \mathrm{MeOH} / \mathrm{CH}_{2} \mathrm{Cl}_{2}\right)$ and recrystallized from $\mathrm{MeOH}$ to afford triazole hybrid $100(342 \mathrm{mg}, 67 \%)$ as a white powdery solid. m.p. $172-174{ }^{\circ} \mathrm{C}$. TLC $R_{\mathrm{f}}$ $=0.30\left(5 \% \mathrm{MeOH} / \mathrm{CH}_{2} \mathrm{Cl}_{2}\right)$. IR $v_{\max }$ (neat) $/ \mathrm{cm}^{-1}: 2941 \mathrm{w}(\mathrm{C}-\mathrm{H} \mathrm{str}), 2843 \mathrm{w}(\mathrm{C}-\mathrm{H} \mathrm{str}), 1737 \mathrm{w}, 1643 \mathrm{~s}$ (C=O str), 1599s ( $\mathrm{C}=\mathrm{C}$ str), 1575s ( $\mathrm{C}=\mathrm{C}$ str), $1515 \mathrm{~m}$ (C=C str), 1465m, 1416m, 1322m, 1261m, 1231s, 1216m, 1192m, 1149s, 1125s, 1027s. ${ }^{1} \mathrm{H}-\mathrm{NMR}\left(500 \mathrm{MHz}, \mathrm{CDCl}_{3}\right): \delta 3.85\left(3 \mathrm{H}, \mathrm{s},-\mathrm{OCH}_{3}\right), 3.88(3 \mathrm{H}$, $\left.\mathrm{s},-\mathrm{OCH}_{3}\right), 3.92\left(6 \mathrm{H}, \mathrm{s}, 2 \times-\mathrm{OCH}_{3}\right), 4.35\left(2 \mathrm{H}, \mathrm{t}, J=5.2 \mathrm{~Hz},-\mathrm{OCH}_{2} \mathrm{CH}_{2} \mathrm{~N}-\right), 4.72(2 \mathrm{H}, \mathrm{t}, J=5.2 \mathrm{~Hz}$, $\left.-\mathrm{OCH}_{2} \mathrm{CH}_{2} \mathrm{~N}-\right), 5.35\left(2 \mathrm{H}, \mathrm{s},-\mathrm{OCH}_{2} \mathrm{CN}-\right), 6.16(2 \mathrm{H}, \mathrm{s}, \mathrm{ArH}), 6.84(1 \mathrm{H}, \mathrm{d}, J=8.8 \mathrm{~Hz}, \mathrm{ArH}), 7.38-7.44(4 \mathrm{H}$, $\mathrm{m}, \mathrm{ArH}), 7.50(1 \mathrm{H}, \mathrm{d}, J=8.4 \mathrm{~Hz}, \mathrm{ArH}), 7.60-7.62(2 \mathrm{H}, \mathrm{m}, \mathrm{ArH}), 7.70(1 \mathrm{H}, \mathrm{t}, J=8.8 \mathrm{~Hz}, \mathrm{ArH}), 7.87$ $(1 \mathrm{H}, \mathrm{d}, J=16.0 \mathrm{~Hz},-\mathrm{CH}=\mathrm{CHCO}-), 7.94(1 \mathrm{H}, \mathrm{s}-\mathrm{CHN}-), 7.95-7.98(2 \mathrm{H}, \mathrm{m}, \mathrm{ArH}), 8.25(1 \mathrm{H}, \mathrm{d}, J=16.0$ $\mathrm{Hz},-\mathrm{CH}=\mathrm{CHCO}-), 8.29(1 \mathrm{H}, \mathrm{dd}, J=8.4,1.6 \mathrm{~Hz}, \mathrm{ArH}) .{ }^{13} \mathrm{C}-\mathrm{NMR}\left(500 \mathrm{MHz}, \mathrm{CDCl}_{3}\right): \delta 49.4,55.4,55.8$, $55.8,65.0,67.3,90.5,106.5,111.7,112.3,118.1,121.6,122.3,124.1,124.7,125.2,125.7,128.2,128.7,130.6$, $130.7,133.5,133.5,135.6,139.3,143.8,149.4,150.7,155.2,156.4,161.6,163.0,175.0,190.4$. LCMS (ES+) $m / z=690.2\left([\mathrm{M}+\mathrm{H}]^{+}, t_{\mathrm{R}}=1.73 \mathrm{~min}\right)$. HRMS (ESI +$) m / z=690.2476[\mathrm{M}+\mathrm{H}]^{+}$found, $\mathrm{C}_{39} \mathrm{H}_{36} \mathrm{O}_{9} \mathrm{~N}_{3}{ }^{+}$ required 690.2446 .

(E)-6-((1-(2-(4-(3-(3,4-Dimethoxyphenyl)acryloyl)-2-methoxyphenoxy)ethyl)-1H-1,2,3-triazol-4-yl)methoxy)-2phenyl-4H-chromen-4-one (101). A mixture of alkyne flavone 31 (179 $\mathrm{mg}, 0.648 \mathrm{mmol})$, azide chalcone 67 ( $246 \mathrm{mg}, 0.641 \mathrm{mmol}), \mathrm{CuSO}_{4} \cdot 5 \mathrm{H}_{2} \mathrm{O}(188 \mathrm{mg}, 0.753 \mathrm{mmol})$ and sodium ascorbate ( $\left.322 \mathrm{mg}, 1.63 \mathrm{mmol}\right)$ in $t-\mathrm{BuOH} / \mathrm{H}_{2} \mathrm{O}(1: 1,40 \mathrm{~mL})$ was reacted according to GP-A. The crude residue was purified by flash column chromatography $\left(\mathrm{SiO}_{2}, 1 \%-5 \% \mathrm{MeOH} / \mathrm{CH}_{2} \mathrm{Cl}_{2}\right)$ and recrystallized from $\mathrm{MeOH}$ to afford triazole hybrid 101 (366 mg, 87\%) as a bright orange crystalline solid. m.p. $128-130{ }^{\circ} \mathrm{C}$. TLC $R_{\mathrm{f}}=0.34$ (5\% MeOH/ $\mathrm{CH}_{2} \mathrm{Cl}_{2}$ ). IR $\gamma_{\max }$ (neat)/ $\mathrm{cm}^{-1}$ : 2940w (C-H str), 2831w (C-H str), 1637s (C=O str), 1618m, $1595 \mathrm{~s}(\mathrm{C}=\mathrm{C}$ str), 1571s (C=C str), 1510s (C=C str), 1481m, 1454s, 1419m, 1360s, 1257s, 1235m, 1198m, 1140s, 1023s. ${ }^{1} \mathrm{H}-\mathrm{NMR}\left(500 \mathrm{MHz}, \mathrm{CDCl}_{3}\right): \delta 3.92\left(3 \mathrm{H}, \mathrm{s},-\mathrm{OCH}_{3}\right), 3.93\left(3 \mathrm{H}, \mathrm{s},-\mathrm{OCH}_{3}\right), 3.94(3 \mathrm{H}, \mathrm{s}$, $\left.-\mathrm{OCH}_{3}\right), 4.47\left(2 \mathrm{H}, \mathrm{t}, J=4.8 \mathrm{~Hz},-\mathrm{OCH}_{2} \mathrm{CH}_{2} \mathrm{~N}-\right), 4.86\left(2 \mathrm{H}, \mathrm{t}, J=4.8 \mathrm{~Hz},-\mathrm{OCH}_{2} \mathrm{CH}_{2} \mathrm{~N}-\right), 5.29(2 \mathrm{H}, \mathrm{s}$, $\left.-\mathrm{OCH}_{2} \mathrm{CN}-\right), 6.79(1 \mathrm{H}, \mathrm{s},-\mathrm{C}=\mathrm{CH}), 6.85(1 \mathrm{H}, \mathrm{d}, J=8.8 \mathrm{~Hz}, \mathrm{ArH}), 6.89(1 \mathrm{H}, \mathrm{d}, J=8.4 \mathrm{~Hz}, \mathrm{ArH}), 7.14(1 \mathrm{H}$, $\mathrm{d}, J=1.6 \mathrm{~Hz}, \mathrm{ArH}), 7.22(1 \mathrm{H}, \mathrm{dd}, J=8.4,2.0 \mathrm{~Hz}, \mathrm{ArH}), 7.33(1 \mathrm{H}, \mathrm{dd}, J=9.2,3.2 \mathrm{~Hz}, \mathrm{ArH}), 7.36(1 \mathrm{H}, \mathrm{d}$, $J=15.2 \mathrm{~Hz},-\mathrm{CH}=\mathrm{CHCO}-), 7.49-7.52(4 \mathrm{H}, \mathrm{m}, \mathrm{ArH}), 7.59-7.61(2 \mathrm{H}, \mathrm{m}, \mathrm{ArH}), 7.71(1 \mathrm{H}, \mathrm{d}, J=3.2 \mathrm{~Hz}$, $\mathrm{ArH}), 7.73(1 \mathrm{H}, \mathrm{d}, J=16.0 \mathrm{~Hz},-\mathrm{CH}=\mathrm{CHCO}-), 7.88-7.91(2 \mathrm{H}, \mathrm{m}, \mathrm{ArH}), 8.04(1 \mathrm{H}, \mathrm{s},-\mathrm{CHN}-) .{ }^{13} \mathrm{C}-\mathrm{NMR}$ (500 MHz, $\mathrm{CDCl}_{3}$ ): $\delta 49.7,55.9,56.0,62.3,67.5,106.3,106.7,110.1,111.1,111.5,112.3,119.4,119.7,122.5$, 123.0, 123.9, 124.5, 124.6, 126.2, 127.8, 129.0, 131.5, 131.7, 132.7, 143.3, 144.5, 149.2, 149.7, 151.2, 151.2, 151.3, 155.5, 163.2, 178.1, 188.5. LCMS (ES+) $m / z=660.2\left([\mathrm{M}+\mathrm{H}]^{+}, t_{\mathrm{R}}=1.81 \mathrm{~min}\right)$. HRMS (ESI+) $m / z=682.2153[\mathrm{M}+\mathrm{Na}]^{+}$found, $\mathrm{C}_{38} \mathrm{H}_{33} \mathrm{O}_{8} \mathrm{~N}_{3} \mathrm{Na}^{+}$required 682.2160 .

(E)-6-((1-(2-(2-Methoxy-4-(3-(2,4,6-trimethoxyphenyl)acryloyl)phenoxy)ethyl)-1H-1,2,3-triazol-4-yl)methoxy)2-phenyl-4H-chromen-4-one (102). A mixture of alkyne flavone 31 (211 $\mathrm{mg}, 0.763 \mathrm{mmol})$, azide chalcone $69(317 \mathrm{mg}, 0.767 \mathrm{mmol}), \mathrm{CuSO}_{4} \cdot 5 \mathrm{H}_{2} \mathrm{O}(211 \mathrm{mg}, 0.843 \mathrm{mmol})$ and sodium ascorbate $(382 \mathrm{mg}$, $1.93 \mathrm{mmol})$ in $t-\mathrm{BuOH} / \mathrm{H}_{2} \mathrm{O}(1: 1,40 \mathrm{~mL})$ was reacted according to GP-A. The crude residue was purified by flash column chromatography $\left(\mathrm{SiO}_{2}, 1 \%-5 \% \mathrm{MeOH} / \mathrm{CH}_{2} \mathrm{Cl}_{2}\right)$ and recrystallized from $\mathrm{MeOH}$ to afford triazole hybrid $102(397 \mathrm{mg}, 75 \%)$ as a bright yellow-orange flaky solid. m.p. $128-130{ }^{\circ} \mathrm{C}$. TLC $R_{\mathrm{f}}=0.25\left(5 \% \mathrm{MeOH} / \mathrm{CH}_{2} \mathrm{Cl}_{2}\right)$. IR $v_{\max }$ (neat) $/ \mathrm{cm}^{-1}: 2941 \mathrm{w}(\mathrm{C}-\mathrm{H} \mathrm{str}), 2838 \mathrm{w}$ (C-H str), 1739w, 1640s (C=O str), 1598s (C=C str), 1569s (C=C str), 1517m (C=C str), 1455s, 1363m, $1320 \mathrm{~m}, 1279 \mathrm{~m}, 1261 \mathrm{~m}, 1201 \mathrm{~s}, 1154 \mathrm{~s}, 1122 \mathrm{~s}, 1035 \mathrm{~s}, 1025 \mathrm{~s} .{ }^{1} \mathrm{H}-\mathrm{NMR}\left(500 \mathrm{MHz}, \mathrm{CDCl}_{3}\right): \delta 3.84(3 \mathrm{H}, \mathrm{s}$, $\left.-\mathrm{OCH}_{3}\right), 3.89\left(6 \mathrm{H}, \mathrm{s}, 2 \times-\mathrm{OCH}_{3}\right), 3.92\left(3 \mathrm{H}, \mathrm{s},-\mathrm{OCH}_{3}\right), 4.45\left(2 \mathrm{H}, \mathrm{t}, J=4.8 \mathrm{~Hz},-\mathrm{OCH}_{2} \mathrm{CH}_{2} \mathrm{~N}-\right), 4.85(2 \mathrm{H}, \mathrm{t}$, $\left.J=4.8 \mathrm{~Hz},-\mathrm{OCH}_{2} \mathrm{CH}_{2} \mathrm{~N}-\right), 5.28\left(2 \mathrm{H}, \mathrm{s},-\mathrm{OCH}_{2} \mathrm{CN}-\right), 6.12(2 \mathrm{H}, \mathrm{s}, \mathrm{ArH}), 6.79(1 \mathrm{H}, \mathrm{s},-\mathrm{C}=\mathrm{CH}), 6.84(1 \mathrm{H}$, 
$\mathrm{d}, J=8.4 \mathrm{~Hz}, \mathrm{ArH}), 7.33(1 \mathrm{H}, \mathrm{dd}, J=9.2,3.2 \mathrm{~Hz}, \mathrm{ArH}), 7.48-7.51(4 \mathrm{H}, \mathrm{m}, \mathrm{ArH}), 7.57(1 \mathrm{H}, \mathrm{dd}, J=8.0$, $1.6 \mathrm{~Hz}, \mathrm{ArH}), 7.61(1 \mathrm{H}, \mathrm{d}, J=1.6 \mathrm{~Hz}, \mathrm{ArH}), 7.71(1 \mathrm{H}, \mathrm{d}, J=2.8 \mathrm{~Hz}, \mathrm{ArH}), 7.84(1 \mathrm{H}, \mathrm{d}, J=16.0 \mathrm{~Hz}$, -CH=CHCO-), 7.88-7.91 (2H, m, ArH), $8.06(1 \mathrm{H}, \mathrm{s},-\mathrm{CHN}-), 8.21(1 \mathrm{H}, \mathrm{d}, J=15.6 \mathrm{~Hz},-\mathrm{CH}=\mathrm{CHCO}-)$. ${ }^{13} \mathrm{C}-\mathrm{NMR}\left(500 \mathrm{MHz}, \mathrm{CDCl}_{3}\right): \delta 49.7,55.3,55.7,55.9,62.3,67.5,90.5,106.2,106.5,106.7,111.6,112.4$, 119.6, 121.4, 122.3, 123.9, 124.4, 124.6, 126.2, 128.9, 131.5, 131.7, 133.6, 135.5, 143.3, 149.4, 150.6, 151.2, 155.6, 161.6, 163.0, 163.2, 178.1, 190.3. LCMS (ES+) $m / z=690.3\left([\mathrm{M}+\mathrm{H}]^{+}, t_{\mathrm{R}}=1.88 \mathrm{~min}\right)$. HRMS (ESI+) $m / z=690.2426[\mathrm{M}+\mathrm{H}]^{+}$found, $\mathrm{C}_{39} \mathrm{H}_{36} \mathrm{O}_{9} \mathrm{~N}_{3}{ }^{+}$required 690.2446.

(E)-6-((1-(2-(2-Methoxy-4-(3-(2,3,4-trimethoxyphenyl)acryloyl)phenoxy)ethyl)-1H-1,2,3-triazol-4-yl)-methoxy) -2-phenyl-4H-chromen-4-one (103). A mixture of alkyne flavone 31 (201 $\mathrm{mg}, 0.729 \mathrm{mmol})$, azide chalcone $68(304 \mathrm{mg}, 0.735 \mathrm{mmol}), \mathrm{CuSO}_{4} \cdot 5 \mathrm{H}_{2} \mathrm{O}(208 \mathrm{mg}, 0.831 \mathrm{mmol})$ and sodium ascorbate $(383 \mathrm{mg}$, $1.93 \mathrm{mmol})$ in $t-\mathrm{BuOH} / \mathrm{H}_{2} \mathrm{O}(1: 1,40 \mathrm{~mL})$ was reacted according to GP-A. The crude residue was purified by flash column chromatography $\left(\mathrm{SiO}_{2}, 1 \%-5 \% \mathrm{MeOH} / \mathrm{CH}_{2} \mathrm{Cl}_{2}\right)$ and recrystallized from $\mathrm{MeOH}$ to afford triazole hybrid 103 (382 mg, 76\%) as a pale yellow flaky solid. m.p. $138-140{ }^{\circ} \mathrm{C}$. TLC $R_{\mathrm{f}}=0.32\left(5 \% \mathrm{MeOH} / \mathrm{CH}_{2} \mathrm{Cl}_{2}\right)$. IR $v_{\max }$ (neat) $/ \mathrm{cm}^{-1}: 2939 \mathrm{w}$ (C-H str), 2839w (C-H str), 1740w, 1640s (C=O str), 1619m, 1593m (C=C str), 1573s (C=C str), 1516m (C=C str), 1495m, 1482m, 1455s, 1415m, 1360s, 1259s, 1200m, 1157m, 1095s, 1026s. ${ }^{1} \mathrm{H}-\mathrm{NMR}\left(500 \mathrm{MHz}, \mathrm{CDCl}_{3}\right): \delta 3.89\left(3 \mathrm{H}, \mathrm{s},-\mathrm{OCH}_{3}\right), 3.90(3 \mathrm{H}$, $\left.\mathrm{s},-\mathrm{OCH}_{3}\right), 3.93\left(3 \mathrm{H}, \mathrm{s},-\mathrm{OCH}_{3}\right), 3.94\left(3 \mathrm{H}, \mathrm{s},-\mathrm{OCH}_{3}\right), 4.47\left(2 \mathrm{H}, \mathrm{t}, J=4.8 \mathrm{~Hz},-\mathrm{OCH}_{2} \mathrm{CH}_{2} \mathrm{~N}-\right), 4.86(2 \mathrm{H}, \mathrm{t}$, $\left.J=4.8 \mathrm{~Hz},-\mathrm{OCH}_{2} \mathrm{CH}_{2} \mathrm{~N}-\right), 5.29\left(2 \mathrm{H}, \mathrm{s},-\mathrm{OCH}_{2} \mathrm{CN}-\right), 6.72(1 \mathrm{H}, \mathrm{d}, J=8.8 \mathrm{~Hz}, \mathrm{ArH}), 6.80(1 \mathrm{H}, \mathrm{s},-\mathrm{C}=\mathrm{CH})$, $6.86(1 \mathrm{H}, \mathrm{d}, J=8.0 \mathrm{~Hz}, \mathrm{ArH}), 7.34(1 \mathrm{H}, \mathrm{dd}, J=9.2,3.2 \mathrm{~Hz}, \mathrm{ArH}), 7.37(1 \mathrm{H}, \mathrm{d}, J=8.8 \mathrm{~Hz}, \mathrm{ArH}), 7.49-7.52$ $(4 \mathrm{H}, \mathrm{m}, \mathrm{ArH}), 7.53(1 \mathrm{H}, \mathrm{d}, J=16.0 \mathrm{~Hz},-\mathrm{CH}=\mathrm{CHCO}-), 7.58-7.61(2 \mathrm{H}, \mathrm{m}, \mathrm{ArH}), 7.71(1 \mathrm{H}, \mathrm{d}, J=3.2 \mathrm{~Hz}$, $\mathrm{ArH}), 7.89-7.92(2 \mathrm{H}, \mathrm{m}, \mathrm{ArH}), 7.96(1 \mathrm{H}, \mathrm{d}, J=16.0 \mathrm{~Hz},-\mathrm{CH}=\mathrm{CHCO}-), 8.05(1 \mathrm{H}, \mathrm{s},-\mathrm{CHN}-) .{ }^{13} \mathrm{C}-\mathrm{NMR}$ (500 MHz, $\left.\mathrm{CDCl}_{3}\right): \delta 49.7,56.0,56.0,60.9,61.3,62.3,67.5,106.3,106.8,107.5,111.5,112.3,119.7,120.8$, 122.0, 122.4, 123.8, 123.9, 124.5, 124.6, 126.2, 129.0, 131.5, 131.7, 132.8, 139.6, 142.4, 143.3, 149.6, 151.1, 151.2, 153.7, 155.6, 155.7, 163.2, 178.1, 188.9. LCMS (ES+) $m / z=690.2\left([\mathrm{M}+\mathrm{H}]^{+}, t_{\mathrm{R}}=1.94 \mathrm{~min}\right)$. HRMS (ESI+) $m / z=690.2452[\mathrm{M}+\mathrm{H}]^{+}$found, $\mathrm{C}_{39} \mathrm{H}_{36} \mathrm{~N}_{3} \mathrm{O}_{9}{ }^{+}$required 690.2452 .

(E)-7-((1-(2-(2-Methoxy-4-(3-(2,4,6-trimethoxyphenyl)acryloyl)phenoxy)ethyl)-1H-1,2,3-triazol-4-yl)-methoxy) -2-phenyl-4H-chromen-4-one (104). A mixture of alkyne flavone 32 (202 $\mathrm{mg}, 0.730 \mathrm{mmol})$, azide chalcone $69(304 \mathrm{mg}, 0.735 \mathrm{mmol}), \mathrm{CuSO}_{4} \cdot 5 \mathrm{H}_{2} \mathrm{O}(218 \mathrm{mg}, 0.872 \mathrm{mmol})$ and sodium ascorbate $(386 \mathrm{mg}$, $1.95 \mathrm{mmol})$ in $t-\mathrm{BuOH} / \mathrm{H}_{2} \mathrm{O}(1: 1,40 \mathrm{~mL})$ was reacted according to GP-A. The crude residue was purified by flash column chromatography $\left(\mathrm{SiO}_{2}, 1 \%-5 \% \mathrm{MeOH} / \mathrm{CH}_{2} \mathrm{Cl}_{2}\right)$ and recrystallized from $\mathrm{MeOH}$ to afford triazole hybrid $104(324 \mathrm{mg}, 64 \%)$ as a pale yellow-white powdery solid. m.p. $168-170{ }^{\circ} \mathrm{C}$. TLC $R_{\mathrm{f}}=0.25\left(5 \% \mathrm{MeOH} / \mathrm{CH}_{2} \mathrm{Cl}_{2}\right)$. IR $v_{\max }$ (neat) $/ \mathrm{cm}^{-1}: 2941 \mathrm{w}(\mathrm{C}-\mathrm{H} \mathrm{str}), 2841 \mathrm{w}$ (C-H str), 1736w, 1634s (C=O str), 1599s (C=C str), 1566s (C=C str), 1512m (C=C str), 1451m, 1441m, 1336s, 1302w, 1279m, 1261m, 1204m, 1175m, 1160w, 1122s, 1092w, 1053w, 1037m, 1016w. ${ }^{1} \mathrm{H}-\mathrm{NMR}$ $\left(500 \mathrm{MHz}, \mathrm{CDCl}_{3}\right): \delta 3.87\left(3 \mathrm{H}, \mathrm{s},-\mathrm{OCH}_{3}\right), 3.91\left(6 \mathrm{H}, \mathrm{s}, 2 \times-\mathrm{OCH}_{3}\right), 3.93\left(3 \mathrm{H}, \mathrm{s},-\mathrm{OCH}_{3}\right), 4.48(2 \mathrm{H}, \mathrm{t}$, $\left.J=5.2 \mathrm{~Hz},-\mathrm{OCH}_{2} \mathrm{CH}_{2} \mathrm{~N}-\right), 4.87\left(2 \mathrm{H}, \mathrm{t}, J=4.8 \mathrm{~Hz},-\mathrm{OCH}_{2} \mathrm{CH}_{2} \mathrm{~N}-\right), 5.35\left(2 \mathrm{H}, \mathrm{s},-\mathrm{OCH}_{2} \mathrm{CN}-\right), 6.14(2 \mathrm{H}$, $\mathrm{s}, \mathrm{ArH}), 6.77(1 \mathrm{H}, \mathrm{s},-\mathrm{C}=\mathrm{CH}), 6.86(1 \mathrm{H}, \mathrm{d}, J=8.0 \mathrm{~Hz}, \mathrm{ArH}), 7.06(1 \mathrm{H}, \mathrm{dd}, J=8.8,2.0 \mathrm{~Hz}, \mathrm{ArH}), 7.14$ $(1 \mathrm{H}, \mathrm{d}, J=2.0 \mathrm{~Hz}, \mathrm{ArH}), 7.49-7.53(3 \mathrm{H}, \mathrm{m}, \mathrm{ArH}), 7.59(1 \mathrm{H}, \mathrm{dd}, J=8.4,2.0 \mathrm{~Hz}, \mathrm{ArH}), 7.63(1 \mathrm{H}, \mathrm{d}$, $J=1.6 \mathrm{~Hz}, \mathrm{ArH}), 7.84(1 \mathrm{H}, \mathrm{d}, J=16.0 \mathrm{~Hz},-\mathrm{CH}=\mathrm{CHCO}-), 7.90-7.92(2 \mathrm{H}, \mathrm{m}, \mathrm{ArH}), 8.07(1 \mathrm{H}, \mathrm{s},-\mathrm{CHN}-)$, $8.15(1 \mathrm{H}, \mathrm{d}, J=8.8 \mathrm{~Hz}, \mathrm{ArH}), 8.23(1 \mathrm{H}, \mathrm{d}, J=16.0 \mathrm{~Hz},-\mathrm{CH}=\mathrm{CHCO}-) .{ }^{13} \mathrm{C}-\mathrm{NMR}\left(500 \mathrm{MHz}, \mathrm{CDCl}_{3}\right): \delta$ 49.8, 55.3, 55.7, 55.8, 62.3, 67.4, 90.4, 101.4, 106.4, 107.3, 111.6, 112.4, 114.8, 118.0, 121.3, 122.3, 124.7, 126.1, 127.0, 128.9, 131.4, 131.6, 133.6, 135.6, 143.0, 149.4, 150.5, 157.8, 161.6, 162.6, 163.0, 163.1, 177.7, 190.2. LCMS (ES+) $m / z=690.2\left([\mathrm{M}+\mathrm{H}]^{+}, t_{\mathrm{R}}=1.70 \mathrm{~min}\right)$. HRMS (ESI+) $m / z=690.2471[\mathrm{M}+\mathrm{H}]^{+}$ found, $\mathrm{C}_{39} \mathrm{H}_{36} \mathrm{O}_{9} \mathrm{~N}_{3}{ }^{+}$required 690.2446 .

(E)-2-(4-(2-(4-((2-(3-(Ferrocenyl)acryloyl)phenoxy)methyl)-1H-1,2,3-triazol-1-yl)ethoxy)phenyl)-3-hydroxy-7methoxy-4H-chromen-4-one (105). A mixture of alkyne chalcone 26 (310 mg, $0.838 \mathrm{mmol})$, azide flavonol 80 (296 mg, $0.838 \mathrm{mmol}), \mathrm{CuSO}_{4} \cdot 5 \mathrm{H}_{2} \mathrm{O}(273 \mathrm{mg}, 1.09 \mathrm{mmol})$ and sodium ascorbate (496 mg, $\left.2.50 \mathrm{mmol}\right)$ in $t-\mathrm{BuOH} / \mathrm{H}_{2} \mathrm{O}(1: 1,40 \mathrm{~mL})$ was reacted according to GP-A. The crude residue was purified by flash column chromatography $\left(\mathrm{SiO}_{2}, 1 \%-5 \% \mathrm{MeOH} / \mathrm{CH}_{2} \mathrm{Cl}_{2}\right)$ to afford triazole hybrid $105(238 \mathrm{mg}, 40 \%)$ as 
a dark red powdery solid. m.p. $118-120^{\circ} \mathrm{C}$. TLC $R_{\mathrm{f}}=0.44\left(5 \% \mathrm{MeOH} / \mathrm{CH}_{2} \mathrm{Cl}_{2}\right) . \mathrm{IR} v_{\max }($ neat $) / \mathrm{cm}^{-1}$ : 3340w (O-H str), 3088w (C-H str), 2929w (C-H str), 1735m (C=O str), 1597s (C=C str), 1541m (C=C str), $1506 \mathrm{~m}$ (C=C str), 1484m, 1449m, 1403m, 1235s, 1206s, 1171s, 1117m, 1106m, 1044w, 1025s. ${ }^{1} \mathrm{H}-\mathrm{NMR}$ $\left(500 \mathrm{MHz}, \mathrm{CDCl}_{3}\right): \delta 3.96\left(3 \mathrm{H}, \mathrm{s},-\mathrm{OCH}_{3}\right), 4.12\left(5 \mathrm{H}, \mathrm{s},-\mathrm{C}_{5} \mathrm{H}_{5}\right), 4.35\left(2 \mathrm{H}, \mathrm{t}, J=4.5 \mathrm{~Hz},-\mathrm{OCH}_{2} \mathrm{CH}_{2} \mathrm{~N}-\right)$, $4.43\left(2 \mathrm{H}, \mathrm{t}, J=1.5 \mathrm{~Hz},-\mathrm{C}_{5} \mathrm{H}_{4}\right), 4.48\left(2 \mathrm{H}, \mathrm{t}, J=1.5 \mathrm{~Hz},-\mathrm{C}_{5} \mathrm{H}_{4}\right), 4.67\left(2 \mathrm{H}, \mathrm{t}, J=5.0 \mathrm{~Hz},-\mathrm{OCH}_{2} \mathrm{CH}_{2} \mathrm{~N}-\right), 5.33$ $\left(2 \mathrm{H}, \mathrm{br} \mathrm{s},-\mathrm{OCH}_{2} \mathrm{CN}-\right), 6.95-6.97(4 \mathrm{H}, \mathrm{m}, \mathrm{ArH}$ and $\mathrm{OH}), 6.96(1 \mathrm{H}, \mathrm{d}, J=15.5 \mathrm{~Hz},-\mathrm{CH}=\mathrm{CHCO}-), 7.01(1 \mathrm{H}$, $\mathrm{dd}, J=9.0,2.0 \mathrm{~Hz}, \mathrm{ArH}), 7.09(1 \mathrm{H}, \mathrm{t}, J=7.5 \mathrm{~Hz}, \mathrm{ArH}), 7.14(1 \mathrm{H}, \mathrm{d}, J=8.5 \mathrm{~Hz}, \mathrm{ArH}), 7.46(1 \mathrm{H}, \mathrm{dd}, J=7.5$, $1.0 \mathrm{~Hz}, \mathrm{ArH}), 7.50(1 \mathrm{H}, \mathrm{d}, J=16.0 \mathrm{~Hz},-\mathrm{CH}=\mathrm{CHCO}-), 7.60(1 \mathrm{H}, \mathrm{dd}, J=7.5,1.5 \mathrm{~Hz}, \mathrm{ArH}), 7.77(1 \mathrm{H}, \mathrm{br} \mathrm{s}$, -CHN-), $8.14(1 \mathrm{H}, \mathrm{d}, J=9.0 \mathrm{~Hz}, \mathrm{ArH}), 8.19(2 \mathrm{H}, \mathrm{d}, J=8.5 \mathrm{~Hz}, \mathrm{ArH}) .{ }^{13} \mathrm{C}-\mathrm{NMR}\left(500 \mathrm{MHz}, \mathrm{CDCl}_{3}\right): \delta 49.6$, 55.8, 63.0, 66.1, 68.9, 69.8, 71.3, 79.0, 99.9, 113.1, 114.5, 114.6, 114.7, 121.4, 124.0, 124.7, 126.7, 129.3, 130.1, 130.2, 132.4, 137.4, 144.1, 145.5, 156.3, 157.2, 158.8, 164.2, 172.6, 192.3. LCMS (ES+) $m / z=724.2\left([\mathrm{M}+\mathrm{H}]^{+}\right.$, $t_{\mathrm{R}}=2.01 \mathrm{~min}$ ). HRMS (ESI+) $\mathrm{m} / z=724.1711[\mathrm{M}+\mathrm{H}]^{+}$found, $\mathrm{C}_{40} \mathrm{H}_{34} \mathrm{~N}_{3} \mathrm{O}_{7} \mathrm{Fe}^{+}$required 724.1746.

(E)-2-(4-(2-(4-((4-(3-(Ferrocenyl)acryloyl)-2-methoxyphenoxy)methyl)-1H-1,2,3-triazol-1-yl)ethoxy)phenyl)-3hydroxy-4H-chromen-4-one (106). A mixture of alkyne chalcone 25 (308 $\mathrm{mg}, 0.769 \mathrm{mmol})$, azide flavonol 79 (252 mg, $0.779 \mathrm{mmol}), \mathrm{CuSO}_{4} \cdot 5 \mathrm{H}_{2} \mathrm{O}(209 \mathrm{mg}, 0.835 \mathrm{mmol})$ and sodium ascorbate $(395 \mathrm{mg}$, $1.99 \mathrm{mmol})$ in $t-\mathrm{BuOH} / \mathrm{H}_{2} \mathrm{O}(1: 1,40 \mathrm{~mL})$ was reacted according to GP-A. The crude residue was purified by flash column chromatography $\left(\mathrm{SiO}_{2}, 1 \%-5 \% \mathrm{MeOH} / \mathrm{CH}_{2} \mathrm{Cl}_{2}\right)$ to afford triazole hybrid 106 (212 mg, 38\%) as a dark-red powdery solid. m.p. 158-160 ${ }^{\circ} \mathrm{C}$. TLC $R_{\mathrm{f}}=0.26\left(3 \% \mathrm{MeOH} / \mathrm{CH}_{2} \mathrm{Cl}_{2}\right)$. IR $v_{\max }$ (neat) $/ \mathrm{cm}^{-1}: 3429 \mathrm{w}$ (O-H str), 2943w (C-H str), 2873w (C-H str), 1735m (C=O str), 1645w, $1596 \mathrm{~s}(\mathrm{C}=\mathrm{C}$ str), 1568s (C=C str), 1509s (C=C str), 1469m, 1418m, 1409m, 1348w, 1292w, 1254s, 1200w, $1180 \mathrm{~m}, 1151 \mathrm{~m}, 1108 \mathrm{~m}, 1027 \mathrm{~m} .{ }^{1} \mathrm{H}-\mathrm{NMR}\left(500 \mathrm{MHz}, \mathrm{CDCl}_{3}\right): \delta 3.97\left(3 \mathrm{H}, \mathrm{s},-\mathrm{OCH}_{3}\right), 4.17\left(5 \mathrm{H}, \mathrm{s},-\mathrm{C}_{5} \mathrm{H}_{5}\right)$, 4.46-4.48 $\left(4 \mathrm{H}, \mathrm{m},-\mathrm{C}_{5} \mathrm{H}_{4}\right.$ and $\left.-\mathrm{OCH}_{2} \mathrm{CH}_{2} \mathrm{~N}-\right), 4.57\left(2 \mathrm{H}, \mathrm{t}, J=2.0 \mathrm{~Hz},-\mathrm{C}_{5} \mathrm{H}_{4}\right), 4.83(2 \mathrm{H}, \mathrm{t}, J=5.0 \mathrm{~Hz}$, $\left.-\mathrm{OCH}_{2} \mathrm{CH}_{2} \mathrm{~N}-\right), 5.43\left(2 \mathrm{H}, \mathrm{s},-\mathrm{OCH}_{2} \mathrm{CN}-\right), 6.94(1 \mathrm{H}, \mathrm{br} \mathrm{s}, \mathrm{OH}), 6.98(2 \mathrm{H}, \mathrm{d}, J=9.0 \mathrm{~Hz}, \mathrm{ArH}), 7.12(1 \mathrm{H}, \mathrm{d}$, $J=15.0 \mathrm{~Hz},-\mathrm{CH}=\mathrm{CHCO}-), 7.15(1 \mathrm{H}, \mathrm{d}, J=8.5 \mathrm{~Hz}, \mathrm{ArH}), 7.43(1 \mathrm{H}, \mathrm{t}, J=8.0 \mathrm{~Hz}, \mathrm{ArH}), 7.57(1 \mathrm{H}, \mathrm{dd}$, $J=8.0,1.5 \mathrm{~Hz}, \mathrm{ArH}), 7.59(1 \mathrm{H}, \mathrm{d}, J=8.5 \mathrm{~Hz}, \mathrm{ArH}), 7.63(1 \mathrm{H}, \mathrm{d}, J=2.0 \mathrm{~Hz}, \mathrm{ArH}), 7.71(1 \mathrm{H}, \mathrm{t}, J=8.0 \mathrm{~Hz}$, $\mathrm{ArH}), 7.73(1 \mathrm{H}, \mathrm{d}, J=15.5 \mathrm{~Hz},-\mathrm{CH}=\mathrm{CHCO}-), 7.90(1 \mathrm{H}, \mathrm{br} \mathrm{s},-\mathrm{CHN}-), 8.22(2 \mathrm{H}, \mathrm{d}, J=9.0 \mathrm{~Hz}, \mathrm{ArH}), 8.25$ $(1 \mathrm{H}, \mathrm{dd}, J=8.0,1.5 \mathrm{~Hz}, \mathrm{ArH}) .{ }^{13} \mathrm{C}-\mathrm{NMR}\left(500 \mathrm{MHz}, \mathrm{CDCl}_{3}\right): \delta 49.7,56.1,62.9,66.3,68.9,69.7,71.2,79.3$, 111.2, 112.3, 114.5, 118.2, 118.5, 120.7, 122.4, 124.3, 124.5, 124.6, 125.4, 129.6, 132.3, 133.5, 137.8, 143.8, $144.7,146.0,149.5,151.3,155.2,159.0,173.2,187.9$. LCMS (ES+) $m / z=724.2\left([\mathrm{M}+\mathrm{H}]^{+}, t_{\mathrm{R}}=2.04 \mathrm{~min}\right)$. HRMS (ESI+) $m / z=724.1723[\mathrm{M}+\mathrm{H}]^{+}$found, $\mathrm{C}_{40} \mathrm{H}_{34} \mathrm{~N}_{3} \mathrm{O}_{7} \mathrm{Fe}^{+}$required 724.1746.

(E)-2-(3-(2-(4-((4-Bromo-2-(3-(1-methyl-1H-indol-3-yl)acryloyl)phenoxy)methyl)-1H-1,2,3-triazol-1-yl)-ethoxy) -4-methoxyphenyl)-3-hydroxy-4H-chromen-4-one (107). A mixture of alkyne chalcone 21 (339 mg, $0.859 \mathrm{mmol})$, azide flavonol $82(301 \mathrm{mg}, 0.853 \mathrm{mmol}), \mathrm{CuSO}_{4} \cdot 5 \mathrm{H}_{2} \mathrm{O}(542 \mathrm{mg}, 2.17 \mathrm{mmol})$ and sodium ascorbate $(202 \mathrm{mg}, 1.02 \mathrm{mmol})$ in $t-\mathrm{BuOH} / \mathrm{H}_{2} \mathrm{O}(1: 1,40 \mathrm{~mL})$ was reacted according to GP-A. The crude residue was purified by flash column chromatography $\left(\mathrm{SiO}_{2}, 1 \%-5 \% \mathrm{MeOH} / \mathrm{CH}_{2} \mathrm{Cl}_{2}\right)$ and recrystallized from $\mathrm{MeOH}$ to afford triazole hybrid $107(258 \mathrm{mg}, 40 \%)$ as a brown powdery solid. m.p. $158-160{ }^{\circ} \mathrm{C}$. TLC $R_{\mathrm{f}}=0.44\left(5 \% \mathrm{MeOH} / \mathrm{CH}_{2} \mathrm{Cl}_{2}\right)$. IR $v_{\max }$ (neat) $/ \mathrm{cm}^{-1}: 3351 \mathrm{w}(\mathrm{O}-\mathrm{H} \mathrm{str}), 2920 \mathrm{~m}(\mathrm{C}-\mathrm{H}$ str), 2852w (C-H str), 1640m (C=O str), 1589s (C=C str), 1559m (C=C str), 1514m (C=C str), 1492m, 1471m, 1397m, 1374m, 1333w, 1269s, 1204m, 1180m, 1130s, 1074w, 1047m, 1020m. ${ }^{1} \mathrm{H}-\mathrm{NMR}(500 \mathrm{MHz}$, $\left.\mathrm{CDCl}_{3}\right): \delta 3.64\left(3 \mathrm{H}, \mathrm{s},-\mathrm{NCH}_{3}\right), 3.83\left(3 \mathrm{H}, \mathrm{s},-\mathrm{OCH}_{3}\right), 4.02\left(2 \mathrm{H}, \mathrm{t}, J=4.5 \mathrm{~Hz},-\mathrm{OCH}_{2} \mathrm{CH}_{2} \mathrm{~N}-\right), 4.49(2 \mathrm{H}, \mathrm{t}$, $\left.J=4.5 \mathrm{~Hz},-\mathrm{OCH}_{2} \mathrm{CH}_{2} \mathrm{~N}-\right), 5.36\left(2 \mathrm{H}, \mathrm{br} \mathrm{s},-\mathrm{OCH}_{2} \mathrm{CN}-\right), 6.96(1 \mathrm{H}, \mathrm{d}, J=9.0 \mathrm{~Hz}, \mathrm{ArH}), 7.02(1 \mathrm{H}, \mathrm{br} \mathrm{s}, \mathrm{OH})$, $7.07(1 \mathrm{H}, \mathrm{d}, J=8.5 \mathrm{~Hz}, \mathrm{ArH}), 7.10-7.13(1 \mathrm{H}, \mathrm{m}, \mathrm{ArH}), 7.18(1 \mathrm{H}, \mathrm{s}, \mathrm{ArH}), 7.23(2 \mathrm{H}, \mathrm{d}, J=3.5 \mathrm{~Hz}, \mathrm{ArH})$, $7.38(1 \mathrm{H}, \mathrm{d}, J=15.5 \mathrm{~Hz},-\mathrm{CH}=\mathrm{CHCO}-), 7.45(1 \mathrm{H}, \mathrm{t}, J=7.5 \mathrm{~Hz}, \mathrm{ArH}), 7.50(1 \mathrm{H}, \mathrm{d}, J=1.5 \mathrm{~Hz}, \mathrm{ArH}), 7.53$ $(1 \mathrm{H}, \mathrm{d}, J=7.5 \mathrm{~Hz}, \mathrm{ArH}), 7.56(1 \mathrm{H}, \mathrm{dd}, J=8.5,2.5 \mathrm{~Hz}, \mathrm{ArH}), 7.60(1 \mathrm{H}, \mathrm{d}, J=8.5 \mathrm{~Hz}, \mathrm{ArH}), 7.73(1 \mathrm{H}, \mathrm{t}$, $J=7.0 \mathrm{~Hz}, \mathrm{ArH}), 7.77(1 \mathrm{H}, \mathrm{d}, J=16.0 \mathrm{~Hz},-\mathrm{CH}=\mathrm{CHCO}-), 7.83(1 \mathrm{H}, \mathrm{d}, J=2.5 \mathrm{~Hz}, \mathrm{ArH}), 7.90(1 \mathrm{H}, \mathrm{dd}$, $J=8.5,1.5 \mathrm{~Hz}, \mathrm{ArH}), 8.06(1 \mathrm{H}, \mathrm{br} \mathrm{s},-\mathrm{CHN}-), 8.27(1 \mathrm{H}, \mathrm{d}, J=8.0 \mathrm{~Hz}, \mathrm{ArH}) .{ }^{13} \mathrm{C}-\mathrm{NMR}\left(500 \mathrm{MHz}, \mathrm{CDCl}_{3}\right)$ :

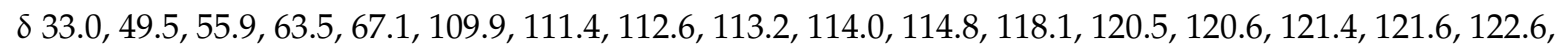
123.0, 123.3, 123.7, 124.6, 124.8, 125.4, 125.7, 131.9, 133.3, 133.6, 134.8, 134.9, 137.7, 137.8, 138.0, 144.5, 
146.9, 151.3, 155.1, 155.8, 173.0, 190.5. LCMS (ES+) $m / z=749.2\left([\mathrm{M}+\mathrm{H}]^{+}, t_{\mathrm{R}}=1.99 \mathrm{~min}\right)$. HRMS (ESI+) $m / z=769.1232[\mathrm{M}+\mathrm{Na}]^{+}$found, $\mathrm{C}_{39} \mathrm{H}_{31} \mathrm{O}_{7} \mathrm{~N}_{4} \mathrm{BrNa}^{+}$required 769.1268 .

(E)-2-(4-(2-(4-((4-Bromo-2-(3-(furan-2-yl)acryloyl)phenoxy)methyl)-1H-1,2,3-triazol-1-yl)ethoxy)-3-methoxyph enyl)-3-hydroxy-4H-chromen-4-one (108). A mixture of alkyne chalcone 24 (289 $\mathrm{mg}, 0.872 \mathrm{mmol}$ ), azide flavonol 81 ( $304 \mathrm{mg}, 0.860 \mathrm{mmol}), \mathrm{CuSO}_{4} \cdot 5 \mathrm{H}_{2} \mathrm{O}(234 \mathrm{mg}, 0.936 \mathrm{mmol})$ and sodium ascorbate $(476 \mathrm{mg}$, $2.40 \mathrm{mmol})$ in $t-\mathrm{BuOH} / \mathrm{H}_{2} \mathrm{O}(1: 1,40 \mathrm{~mL})$ was reacted according to GP-A. The crude residue was purified by flash column chromatography $\left(\mathrm{SiO}_{2}, 1 \%-5 \% \mathrm{MeOH} / \mathrm{CH}_{2} \mathrm{Cl}_{2}\right)$ and recrystallized from $\mathrm{MeOH}$ to afford triazole hybrid $108(245 \mathrm{mg}, 42 \%)$ as a dark yellow-brown powdery solid. m.p. $118-120^{\circ} \mathrm{C}$. TLC $R_{\mathrm{f}}=0.41\left(5 \% \mathrm{MeOH} / \mathrm{CH}_{2} \mathrm{Cl}_{2}\right)$. IR $v_{\max }$ (neat) $/ \mathrm{cm}^{-1}: 3275 \mathrm{w}(\mathrm{O}-\mathrm{H}$ str), $3110 \mathrm{w}(\mathrm{C}-\mathrm{H}$ str), 2926w (C-H str), 1650m (C=O str), 1598s (C=C str), 1549m (C=C str), 1515s (C=C str), 1481s, 1422w, $1398 \mathrm{~m}, 1267 \mathrm{~s}, 1232 \mathrm{~m}, 1207 \mathrm{~s}, 1177 \mathrm{~m}, 1145 \mathrm{~s}, 1111 \mathrm{~m}, 1041 \mathrm{~m}, 1017 \mathrm{~m}, 1008 \mathrm{~m} .{ }^{1} \mathrm{H}-\mathrm{NMR}\left(500 \mathrm{MHz}, \mathrm{CDCl}_{3}\right)$ : $\delta 3.90\left(3 \mathrm{H}, \mathrm{s},-\mathrm{OCH}_{3}\right), 4.43\left(2 \mathrm{H}, \mathrm{t}, J=4.0 \mathrm{~Hz},-\mathrm{OCH}_{2} \mathrm{CH}_{2} \mathrm{~N}-\right), 4.75\left(2 \mathrm{H}, \mathrm{t}, J=4.0 \mathrm{~Hz},-\mathrm{OCH}_{2} \mathrm{CH}_{2} \mathrm{~N}-\right)$, $5.33\left(2 \mathrm{H}, \mathrm{br} \mathrm{s},-\mathrm{OCH}_{2} \mathrm{CN}-\right), 6.47(1 \mathrm{H}, \mathrm{d}, J=1.5 \mathrm{~Hz}, \mathrm{ArH}), 6.64(1 \mathrm{H}, \mathrm{d}, J=3.0 \mathrm{~Hz}, \mathrm{ArH}), 6.89(1 \mathrm{H}, \mathrm{d}$, $J=8.5 \mathrm{~Hz}, \mathrm{ArH}), 7.01(1 \mathrm{H}, \mathrm{s}, \mathrm{ArH}), 7.06-7.08(1 \mathrm{H}, \mathrm{m}, \mathrm{ArH}), 7.25(1 \mathrm{H}, \mathrm{d}, J=16.0 \mathrm{~Hz},-\mathrm{CH}=\mathrm{CHCO}-$ overlain by $\left.\mathrm{CDCl}_{3}\right), 7.36(1 \mathrm{H}, \mathrm{d}, J=15.5 \mathrm{~Hz},-\mathrm{CH}=\mathrm{CHCO}-), 7.44(1 \mathrm{H}, \mathrm{t}, J=7.5 \mathrm{~Hz}, \mathrm{ArH}), 7.52(1 \mathrm{H}, \mathrm{s}$, $\mathrm{ArH}), 7.54(1 \mathrm{H}, \mathrm{d}, J=6.5 \mathrm{~Hz}, \mathrm{ArH}), 7.61(1 \mathrm{H}, \mathrm{d}, J=8.5 \mathrm{~Hz}, \mathrm{ArH}), 7.72(1 \mathrm{H}, \mathrm{d}, J=7.0 \mathrm{~Hz}, \mathrm{ArH}), 7.74(1 \mathrm{H}$, $\mathrm{d}, J=2.0 \mathrm{~Hz}, \mathrm{ArH}), 7.82(1 \mathrm{H}, \mathrm{d}, J=8.5 \mathrm{~Hz}, \mathrm{ArH}), 7.84(1 \mathrm{H}, \mathrm{s},-\mathrm{CHN}-), 8.09(1 \mathrm{H}, \mathrm{br} \mathrm{s}, \mathrm{OH}), 8.26(1 \mathrm{H}, \mathrm{d}$, $J=7.5 \mathrm{~Hz}, \mathrm{ArH}) .{ }^{13} \mathrm{C}-\mathrm{NMR}\left(500 \mathrm{MHz}, \mathrm{CDCl}_{3}\right): \delta 49.9,55.8,63.2,67.5,111.4,112.7,113.6,113.9,115.1$, $116.1,118.2,120.6,121.1,124.0,124.5,125.2,125.4,129.6,131.1,133.0,133.5,135.4,137.9,144.6,145.0$, $148.7,149.4,151.4,155.2,155.7,173.1,190.2$. LCMS (ES +$) m / z=686.2\left([\mathrm{M}+\mathrm{H}]^{+}, t_{\mathrm{R}}=2.00 \mathrm{~min}\right) . \mathrm{HRMS}$ (ESI+) $m / z=684.0955[\mathrm{M}+\mathrm{H}]^{+}$found, $\mathrm{C}_{34} \mathrm{H}_{27} \mathrm{O}_{8} \mathrm{~N}_{3} \mathrm{Br}^{+}$required 684.0976.

6-(2-(4-(((4-Oxo-2-phenyl-4H-chromen-3-yl)oxy)methyl)-1H-1,2,3-triazol-1-yl)ethoxy)-2-phenyl-4H-chromen4-one (109). A mixture of alkyne flavone $30(277 \mathrm{mg}, 1.00 \mathrm{mmol})$, azide flavone 85 (307 mg, $0.998 \mathrm{mmol})$, $\mathrm{CuSO}_{4} \cdot 5 \mathrm{H}_{2} \mathrm{O}(303 \mathrm{mg}, 1.21 \mathrm{mmol})$ and sodium ascorbate $(492 \mathrm{mg}, 2.48 \mathrm{mmol})$ in $t-\mathrm{BuOH} / \mathrm{H}_{2} \mathrm{O}$ (1:1, $40 \mathrm{~mL}$ ) was reacted according to GP-A. The crude residue was purified by flash column chromatography $\left(\mathrm{SiO}_{2}, 1 \%-5 \% \mathrm{MeOH} / \mathrm{CH}_{2} \mathrm{Cl}_{2}\right)$ to afford triazole hybrid $109(534 \mathrm{mg}, 92 \%)$ as a white powdery solid. m.p. $234-236{ }^{\circ} \mathrm{C}$. TLC $R_{\mathrm{f}}=0.36\left(5 \% \mathrm{MeOH} / \mathrm{CH}_{2} \mathrm{Cl}_{2}\right) . \mathrm{IR} v_{\max }$ (neat) $/ \mathrm{cm}^{-1}: 3145 \mathrm{w}$ (C-H str), 2940w (C-H str), 1736w, 1643s (C=O str), 1627s, 1600m, 1570m (C=C str), 1561m (C=C str), 1482m, 1470m, 1455s, 1398m, 1361s, 1293m, 1197s, 1187m, 1148m, 1087m, 1047m, 1026w. ${ }^{1} \mathrm{H}-\mathrm{NMR}$ (500 $\left.\mathrm{MHz}, \mathrm{CDCl}_{3}\right): \delta 4.41\left(2 \mathrm{H}, \mathrm{t}, J=4.8 \mathrm{~Hz},-\mathrm{OCH}_{2} \mathrm{CH}_{2} \mathrm{~N}-\right), 4.73\left(2 \mathrm{H}, \mathrm{t}, J=4.8 \mathrm{~Hz},-\mathrm{OCH}_{2} \mathrm{CH}_{2} \mathrm{~N}-\right), 5.34(2 \mathrm{H}$, s, $\left.-\mathrm{OCH}_{2} \mathrm{CN}-\right), 6.83(1 \mathrm{H}, \mathrm{s},-\mathrm{C}=\mathrm{CH}), 7.25\left(1 \mathrm{H}, \mathrm{dd}, J=9.2,3.2 \mathrm{~Hz}, \mathrm{ArH}\right.$, overlain by $\left.\mathrm{CDCl}_{3}\right), 7.41-7.46$ $(4 \mathrm{H}, \mathrm{m}, \mathrm{ArH}), 7.51-7.55(5 \mathrm{H}, \mathrm{m}, \mathrm{ArH}), 7.58(1 \mathrm{H}, \mathrm{d}, J=3.2 \mathrm{~Hz}, \mathrm{ArH}), 7.70(1 \mathrm{H}, \mathrm{t}, J=7.2 \mathrm{~Hz}, \mathrm{ArH}), 7.81$ $(1 \mathrm{H}, \mathrm{s},-\mathrm{CHN}-), 7.92-7.94(2 \mathrm{H}, \mathrm{m}, \mathrm{ArH}), 8.01-8.04(2 \mathrm{H}, \mathrm{m}, \mathrm{ArH}), 8.29(1 \mathrm{H}, \mathrm{d}, J=7.2 \mathrm{~Hz}, \mathrm{ArH}) .{ }^{13} \mathrm{C}-\mathrm{NMR}$ (500 MHz, $\mathrm{CDCl}_{3}$ ): $\delta 49.4,65.1,66.7,105.9,106.9,118.0,119.9,123.8,124.1,124.5,124.8,125.7,126.2$, 128.3, 128.7, 129.0, 130.7, 131.6, 131.7, 133.5, 139.5, 144.0, 151.4, 155.1, 155.3, 156.4, 163.3, 175.1, 178.0. LCMS (ES+) $m / z=584.1\left([\mathrm{M}+\mathrm{H}]^{+}, t_{\mathrm{R}}=1.91 \mathrm{~min}\right)$. HRMS (ESI+) $m / z=606.1610[\mathrm{M}+\mathrm{Na}]^{+}$found, $\mathrm{C}_{35} \mathrm{H}_{25} \mathrm{O}_{6} \mathrm{~N}_{3} \mathrm{Na}^{+}$required 606.1636.

3-Hydroxy-2-(4-(2-(4-(((4-oxo-2-phenyl-4H-chromen-3-yl)oxy)methyl)-1H-1,2,3-triazol-1-yl)ethoxy)phenyl) -4H-chromen-4-one (110). A mixture of alkyne flavone $30(272 \mathrm{mg}, 0.984 \mathrm{mmol})$, azide flavonol 79 ( $311 \mathrm{mg}, 0.961 \mathrm{mmol}), \mathrm{CuSO}_{4} \cdot 5 \mathrm{H}_{2} \mathrm{O}(274 \mathrm{mg}, 1.10 \mathrm{mmol})$ and sodium ascorbate $(557 \mathrm{mg}, 2.81 \mathrm{mmol})$ in $t$ - $\mathrm{BuOH} / \mathrm{H}_{2} \mathrm{O}(1: 1,40 \mathrm{~mL})$ was reacted according to GP-A. The crude residue was purified by flash column chromatography $\left(\mathrm{SiO}_{2}, 1 \%-5 \% \mathrm{MeOH} / \mathrm{CH}_{2} \mathrm{Cl}_{2}\right)$ and recrystallized from $\mathrm{MeOH}$ to afford triazole hybrid $110(272 \mathrm{mg}, 47 \%)$ as an off-white powdery solid. m.p. $148-150{ }^{\circ} \mathrm{C}$. TLC $R_{\mathrm{f}}=0.34(5 \%$ $\mathrm{MeOH} / \mathrm{CH}_{2} \mathrm{Cl}_{2}$ ). IR $v_{\max }$ (neat)/ $\mathrm{cm}^{-1}: 3254 \mathrm{w}$ (br) (O-H str), 2964w (C-H str), 1602s (C=O str), 1564m $(\mathrm{C}=\mathrm{C}$ str), $1549 \mathrm{~m}$ ( $\mathrm{C}=\mathrm{C}$ str), 1509s (C=C str), 1481m, 1469s, 1427m, 1403s, 1282m, 1250s, 1198m, 1183s, $1150 \mathrm{w}, 1116 \mathrm{~m}, 1109 \mathrm{~m}, 1041 \mathrm{~m} .{ }^{1} \mathrm{H}-\mathrm{NMR}\left(500 \mathrm{MHz}, \mathrm{CDCl}_{3}\right): \delta 4.39\left(2 \mathrm{H}, \mathrm{t}, J=5.5 \mathrm{~Hz},-\mathrm{OCH}_{2} \mathrm{CH}_{2} \mathrm{~N}-\right)$, $4.73\left(2 \mathrm{H}, \mathrm{t}, J=5.5 \mathrm{~Hz},-\mathrm{OCH}_{2} \mathrm{CH}_{2} \mathrm{~N}-\right), 5.33\left(2 \mathrm{H}, \mathrm{s},-\mathrm{OCH}_{2} \mathrm{CN}-\right), 6.99(3 \mathrm{H}, \mathrm{d}, J=9.0 \mathrm{~Hz}, \mathrm{ArH}$ and $\mathrm{OH})$, 7.41-7.46 (5H, m, ArH), 7.53 (1H, dd, J = 8.0, 0.5 Hz, ArH), 7.59 (1H, d, J = 8.0 Hz, ArH), 7.68-7.72 (2H, $\mathrm{m}, \mathrm{ArH}), 7.85(1 \mathrm{H}, \mathrm{s},-\mathrm{CHN}-), 8.01-8.03(2 \mathrm{H}, \mathrm{m}, \mathrm{ArH}), 8.23(2 \mathrm{H}, \mathrm{d}, J=9.0 \mathrm{~Hz}, \mathrm{ArH}), 8.25(1 \mathrm{H}, \mathrm{dd}, J=8.0$, 
$1.5 \mathrm{~Hz}, \mathrm{ArH}), 8.29(1 \mathrm{H}, \mathrm{dd}, J=8.0,1.5 \mathrm{~Hz}, \mathrm{ArH}) .{ }^{13} \mathrm{C}-\mathrm{NMR}\left(500 \mathrm{MHz}, \mathrm{CDCl}_{3}\right): \delta 49.5,65.1,66.2,114.5$, $118.1,118.2$, 120.7, 124.1, 124.5, 124.5, 124.8, 125.0, 125.4, 125.7, 128.4, 128.7, 129.6, 130.7, 133.5, 133.6, 137.8, 139.5, 144.1, 144.8, 155.3, 155.3, 156.4, 159.1, 173.2, 175.1. LCMS (ES+) $m / z=600.0\left([\mathrm{M}+\mathrm{H}]^{+}\right.$, $t_{\mathrm{R}}=1.75 \mathrm{~min}$ ). HRMS (ESI+) $\mathrm{m} / z=600.1755[\mathrm{M}+\mathrm{H}]^{+}$found, $\mathrm{C}_{35} \mathrm{H}_{26} \mathrm{O}_{7} \mathrm{~N}_{3}{ }^{+}$required 600.1765.

3-Hydroxy-2-(4-((1-(2-(4-(3-hydroxy-4-oxo-4H-chromen-2-yl)phenoxy)ethyl)-1H-1,2,3-triazol-4-yl)-methoxy) phenyl)-4H-chromen-4-one (111). A mixture of alkyne flavonol $36(273 \mathrm{mg}, 0.933 \mathrm{mmol})$, azide flavonol 79 ( $310 \mathrm{mg}, 0.958 \mathrm{mmol}), \mathrm{CuSO}_{4} \cdot 5 \mathrm{H}_{2} \mathrm{O}(296 \mathrm{mg}, 1.19 \mathrm{mmol})$ and sodium ascorbate $(461 \mathrm{mg}, 2.33 \mathrm{mmol})$ in $t-\mathrm{BuOH} / \mathrm{H}_{2} \mathrm{O}(1: 1,40 \mathrm{~mL})$ was reacted according to GP-A. The crude residue was purified by flash column chromatography $\left(\mathrm{SiO}_{2}, 1 \%-5 \% \mathrm{MeOH} / \mathrm{CH}_{2} \mathrm{Cl}_{2}\right)$ and recrystallized from $\mathrm{MeOH}$ to afford triazole hybrid $111(82.7 \mathrm{mg}, 14 \%)$ as a dark brown powdery solid. m.p. 218-220 ${ }^{\circ} \mathrm{C}$. TLC $R_{\mathrm{f}}=0.41$ (5\% MeOH/ $\mathrm{CH}_{2} \mathrm{Cl}_{2}$ ). IR $v_{\max }$ (neat) $/ \mathrm{cm}^{-1}: 3284 \mathrm{w}$ (O-H str), 3087w (C-H str), 2924w (C-H str), 1600s ( $C=O$ str), $1563 \mathrm{~m}(\mathrm{C}=\mathrm{C}$ str), $1543 \mathrm{~m}$ ( $\mathrm{C}=\mathrm{C}$ str), 1508s ( $\mathrm{C}=\mathrm{C}$ str), 1491s, 1424s, 1409s, 1248s, $1209 \mathrm{~m}, 1180 \mathrm{~s}$, 1108s, 1043m, 1014w. ${ }^{1} \mathrm{H}-\mathrm{NMR}\left(500 \mathrm{MHz}\right.$, DMSO- $\left.d_{6}\right): \delta 4.53\left(2 \mathrm{H}, \mathrm{t}, J=4.5 \mathrm{~Hz},-\mathrm{OCH}_{2} \mathrm{CH}_{2} \mathrm{~N}-\right), 4.85$ $\left(2 \mathrm{H}, \mathrm{t}, J=4.5 \mathrm{~Hz},-\mathrm{OCH}_{2} \mathrm{CH}_{2} \mathrm{~N}-\right), 5.29\left(2 \mathrm{H}, \mathrm{s},-\mathrm{OCH}_{2} \mathrm{CN}-\right), 7.11(2 \mathrm{H}, \mathrm{d}, J=8.5 \mathrm{~Hz}, \mathrm{ArH}), 7.25(2 \mathrm{H}, \mathrm{d}$, $J=8.5 \mathrm{~Hz}, \mathrm{ArH}), 7.43-7.47(2 \mathrm{H}, \mathrm{m}, \mathrm{ArH}), 7.71-7.80(4 \mathrm{H}, \mathrm{m}, \mathrm{ArH}), 8.09(2 \mathrm{H}, \mathrm{t}, J=6.5 \mathrm{~Hz}, \mathrm{ArH}), 8.17(2 \mathrm{H}$, $\mathrm{d}, J=9.0 \mathrm{~Hz}, \mathrm{ArH}), 8.21(2 \mathrm{H}, \mathrm{d}, J=9.0 \mathrm{~Hz}, \mathrm{ArH}), 8.39(1 \mathrm{H}, \mathrm{s},-\mathrm{CHN}-), 9.47(1 \mathrm{H}, \mathrm{s}, \mathrm{OH}), 9.48(1 \mathrm{H}, \mathrm{s}, \mathrm{OH})$. ${ }^{13} \mathrm{C}-\mathrm{NMR}\left(500 \mathrm{MHz}\right.$, DMSO- $d_{6}$ ): $\delta$ 49.0, 61.1, 66.3, 114.6, 114.8, 115.4, 118.3, 121.3, 123.9, 124.1, 124.5, $124.7,125.4,129.4,129.6,133.4,133.5,138.2,142.4,145.3,145.4,154.4,158.9,159.1,172.6$. LCMS (ES+) $m / z=616.1\left([\mathrm{M}+\mathrm{H}]^{+}, t_{\mathrm{R}}=1.68 \mathrm{~min}\right)$. HRMS (ESI +$) \mathrm{m} / z=616.1722[\mathrm{M}+\mathrm{H}]^{+}$found, $\mathrm{C}_{35} \mathrm{H}_{26} \mathrm{~N}_{3} \mathrm{O}_{8}{ }^{+}$ required 616.1720.

3-(4-Methoxyphenyl)-7-((1-(2-((4-oxo-2-phenyl-4H-chromen-6-yl)oxy)ethyl)-1H-1,2,3-triazol-4-yl)-methoxy)$4 \mathrm{H}$-chromen-4-one (112). A mixture of alkyne isoflavone 46 (280 $\mathrm{mg}, 0.914 \mathrm{mmol})$, azide flavone 85 ( $282 \mathrm{mg}, 0.919 \mathrm{mmol}), \mathrm{CuSO}_{4} \cdot 5 \mathrm{H}_{2} \mathrm{O}(279 \mathrm{mg}, 1.12 \mathrm{mmol})$ and sodium ascorbate $(503 \mathrm{mg}, 2.54 \mathrm{mmol})$ in $t-\mathrm{BuOH} / \mathrm{H}_{2} \mathrm{O}(1: 1,40 \mathrm{~mL})$ was reacted according to GP-A. The crude residue was purified by flash column chromatography $\left(\mathrm{SiO}_{2}, 1 \%-5 \% \mathrm{MeOH} / \mathrm{CH}_{2} \mathrm{Cl}_{2}\right)$ and recrystallized from $\mathrm{MeOH}$ to afford triazole hybrid 112 (531 mg, 95\%) as a white powdery solid. m.p. $234-236{ }^{\circ} \mathrm{C}$. TLC $R_{\mathrm{f}}=(3 \%$ $\mathrm{MeOH} / \mathrm{CH}_{2} \mathrm{Cl}_{2}$ ). IR $v_{\max }$ (neat)/ $\mathrm{cm}^{-1}: 3082 \mathrm{w}$ (C-H str), 2941w (C-H str), 1641s, 1625s (C=O str), $1608 \mathrm{~s}, 1567 \mathrm{~s}$ (C=C str), 1515s (C=C str), 1497w, 1483w, 1456s, 1443s, 1359s, 1292s, 1252s, 1204m, 1192m, $1185 \mathrm{~s}, 1137 \mathrm{w}, 1099 \mathrm{~m}, 1084 \mathrm{~m}, 1047 \mathrm{~s}, 1032 \mathrm{~s} .{ }^{1} \mathrm{H}-\mathrm{NMR}\left(500 \mathrm{MHz}, \mathrm{CDCl}_{3}\right): \delta 3.83\left(3 \mathrm{H}, \mathrm{s},-\mathrm{OCH}_{3}\right), 4.50(2 \mathrm{H}$, $\left.\mathrm{t}, J=4.8 \mathrm{~Hz},-\mathrm{OCH}_{2} \mathrm{CH}_{2} \mathrm{~N}-\right), 4.86\left(2 \mathrm{H}, \mathrm{t}, J=4.8 \mathrm{~Hz},-\mathrm{OCH}_{2} \mathrm{CH}_{2} \mathrm{~N}-\right), 5.36\left(2 \mathrm{H}, \mathrm{s},-\mathrm{OCH}_{2} \mathrm{CN}-\right), 6.82(1 \mathrm{H}, \mathrm{s}$, $-\mathrm{C}=\mathrm{CH}), 6.95(2 \mathrm{H}, \mathrm{d}, J=8.4 \mathrm{~Hz}, \mathrm{ArH}), 7.01(1 \mathrm{H}, \mathrm{d}, J=2.0 \mathrm{~Hz}, \mathrm{ArH}), 7.07(1 \mathrm{H}, \mathrm{dd}, J=8.8,2.0 \mathrm{~Hz}, \mathrm{ArH})$, $7.18(1 \mathrm{H}, \mathrm{dd}, J=8.8,3.2 \mathrm{~Hz}, \operatorname{ArH}), 7.48-7.56(6 \mathrm{H}, \mathrm{m}, \mathrm{ArH}), 7.59(1 \mathrm{H}, \mathrm{d}, J=3.2 \mathrm{~Hz}, \operatorname{ArH}), 7.86(1 \mathrm{H}$, $\mathrm{s},-\mathrm{CHN}-), 7.90-7.92(3 \mathrm{H}, \mathrm{m},-\mathrm{C}=\mathrm{CH}$ and $\mathrm{ArH}), 8.22(1 \mathrm{H}, \mathrm{d}, J=8.4 \mathrm{~Hz}, \mathrm{ArH}) .{ }^{13} \mathrm{C}-\mathrm{NMR}(500 \mathrm{MHz}$, $\left.\mathrm{CDCl}_{3}\right): \delta 49.8,55.3,62.4,66.8,101.3,106.2,106.9,113.9,115.0,118.8,119.9,123.4,124.0,124.1,124.6$, 124.9, 126.3, 127.9, 129.1, 130.1, 131.7, 143.3, 151.5, 152.1, 155.0, 157.7, 159.6, 162.3, 163.5, 175.8, 178.0. LCMS (ES+) $m / z=614.2\left([\mathrm{M}+\mathrm{H}]^{+}, t_{\mathrm{R}}=1.94 \mathrm{~min}\right)$. HRMS (ESI +$) m / z=614.1942[\mathrm{M}+\mathrm{H}]^{+}$found, $\mathrm{C}_{36} \mathrm{H}_{28} \mathrm{~N}_{3} \mathrm{O}_{7}+$ required 614.1927.

(E)-7-Methoxy-3-(4-((1-(2-(2-methoxy-4-(3-(2,4,6-trimethoxyphenyl)acryloyl)phenoxy)ethyl)-1H-1,2,3-triazol4-yl)methoxy)phenyl)-4H-chromen-4-one (113). A mixture of alkyne isoflavone 45 (236 mg, $0.770 \mathrm{mmol}$ ), azide chalcone $69(308 \mathrm{mg}, 0.744 \mathrm{mmol}), \mathrm{CuSO}_{4} \cdot 5 \mathrm{H}_{2} \mathrm{O}(210 \mathrm{mg}, 0.843 \mathrm{mmol})$ and sodium ascorbate $(363 \mathrm{mg}, 1.83 \mathrm{mmol})$ in $t$ - $\mathrm{BuOH} / \mathrm{H}_{2} \mathrm{O}(1: 1,40 \mathrm{~mL})$ was reacted according to GP-A. The crude residue was purified by flash column chromatography $\left(\mathrm{SiO}_{2}, 1 \%-5 \% \mathrm{MeOH} / \mathrm{CH}_{2} \mathrm{Cl}_{2}\right)$ and recrystallized from MeOH/PE to afford triazole hybrid $113(167 \mathrm{mg}, 31 \%)$ as a pale yellow-orange powdery solid. m.p. $118-120^{\circ} \mathrm{C}$. TLC $R_{\mathrm{f}}=0.33\left(5 \% \mathrm{MeOH} / \mathrm{CH}_{2} \mathrm{Cl}_{2}\right)$. IR $v_{\max }$ (neat) $/ \mathrm{cm}^{-1}: 2942 \mathrm{w}$ (C-H str), $2840 \mathrm{w}$ (C-H str), 1637m (C=O str), 1595s (C=C str), 1559s (C=C str), 1511s (C=C str), 1438m, 1418m, 1336m, $1300 \mathrm{~m}, 1244 \mathrm{~s}, 1201 \mathrm{~m}, 1153 \mathrm{~s}, 1177 \mathrm{~s}, 1118 \mathrm{~s}, 1037 \mathrm{~s}, 1024 \mathrm{~s} .{ }^{1} \mathrm{H}-\mathrm{NMR}\left(500 \mathrm{MHz}, \mathrm{CDCl}_{3}\right): \delta 3.86(3 \mathrm{H}, \mathrm{s}$, $\left.-\mathrm{OCH}_{3}\right), 3.90\left(6 \mathrm{H}, \mathrm{s}, 2 \times-\mathrm{OCH}_{3}\right), 3.90\left(3 \mathrm{H}, \mathrm{s},-\mathrm{OCH}_{3}\right), 3.93\left(3 \mathrm{H}, \mathrm{s},-\mathrm{OCH}_{3}\right), 4.46(2 \mathrm{H}, \mathrm{t}, J=5.2 \mathrm{~Hz}$, $\left.-\mathrm{OCH}_{2} \mathrm{CH}_{2} \mathrm{~N}-\right), 4.84\left(2 \mathrm{H}, \mathrm{t}, J=5.2 \mathrm{~Hz},-\mathrm{OCH}_{2} \mathrm{CH}_{2} \mathrm{~N}-\right), 5.26\left(2 \mathrm{H}, \mathrm{s},-\mathrm{OCH}_{2} \mathrm{CN}-\right), 6.13(2 \mathrm{H}, \mathrm{s}, \mathrm{ArH}), 6.83$ $(1 \mathrm{H}, \mathrm{d}, J=0.4 \mathrm{~Hz}, \mathrm{ArH}), 6.84(1 \mathrm{H}, \mathrm{d}, J=6.0 \mathrm{~Hz}, \mathrm{ArH}), 6.97(1 \mathrm{H}, \mathrm{dd}, J=8.8,2.4 \mathrm{~Hz}, \mathrm{ArH}), 7.04(2 \mathrm{H}$, 
$\mathrm{d}, J=8.8 \mathrm{~Hz}, \mathrm{ArH}), 7.48(2 \mathrm{H}, \mathrm{d}, J=8.8 \mathrm{~Hz}, \mathrm{ArH}), 7.58(1 \mathrm{H}, \mathrm{dd}, J=8.4,2.0 \mathrm{~Hz}, \operatorname{ArH}), 7.62(1 \mathrm{H}, \mathrm{d}$, $J=2.0 \mathrm{~Hz}, \mathrm{ArH}), 7.84(1 \mathrm{H}, \mathrm{d}, J=15.6 \mathrm{~Hz},-\mathrm{CH}=\mathrm{CHCO}-), 7.88(1 \mathrm{H}, \mathrm{s},-\mathrm{CHN}-), 8.02(1 \mathrm{H}, \mathrm{s},-\mathrm{C}=\mathrm{CH}), 8.19$ $(1 \mathrm{H}, \mathrm{d}, J=8.8 \mathrm{~Hz}, \mathrm{ArH}), 8.21(1 \mathrm{H}, \mathrm{d}, J=15.6 \mathrm{~Hz},-\mathrm{CH}=\mathrm{CHCO}-) .{ }^{13} \mathrm{C}-\mathrm{NMR}\left(500 \mathrm{MHz}, \mathrm{CDCl}_{3}\right): \delta 49.7$, 55.0, 55.4, 55.8, 55.9, 62.1, 67.6, 90.5, 100.0, 106.6, 111.7, 112.5, 114.5, 114.8, 118.4, 121.6, 122.3, 122.9, 124.4, 127.7, 130.1, 133.7, 135.6, 144.3, 149.5, 150.7, 152.1, 157.9, 158.2, 158.3, 161.6, 163.0, 163.9, 175.7, 190.3. LCMS (ES+) $m / z=720.3\left([\mathrm{M}+\mathrm{H}]^{+}, t_{\mathrm{R}}=1.69 \mathrm{~min}\right)$. HRMS (ESI +$) m / z=720.2529[\mathrm{M}+\mathrm{H}]^{+}$ found, $\mathrm{C}_{40} \mathrm{H}_{38} \mathrm{O}_{10} \mathrm{~N}_{3}{ }^{+}$required 720.2552 .

(E)-7-((1-(2-(2-Methoxy-4-(3-(2,3,4-trimethoxyphenyl)acryloyl)phenoxy)ethyl)-1H-1,2,3-triazol-4-yl)-methoxy)3-(4-methoxyphenyl)-4H-chromen-4-one (114). A mixture of alkyne isoflavone 46 (226 $\mathrm{mg}, 0.738 \mathrm{mmol}$ ), azide chalcone 68 ( $301 \mathrm{mg}, 0.728 \mathrm{mmol}), \mathrm{CuSO}_{4} \cdot 5 \mathrm{H}_{2} \mathrm{O}(204 \mathrm{mg}, 0.818 \mathrm{mmol})$ and sodium ascorbate (393 mg, $1.98 \mathrm{mmol})$ in $t$ - $\mathrm{BuOH} / \mathrm{H}_{2} \mathrm{O}(1: 1,40 \mathrm{~mL})$ was reacted according to GP-A. The crude residue was purified by flash column chromatography $\left(\mathrm{SiO}_{2}, 1 \%-5 \% \mathrm{MeOH} / \mathrm{CH}_{2} \mathrm{Cl}_{2}\right)$ and recrystallized from $\mathrm{MeOH}$ to afford triazole hybrid $114(331 \mathrm{mg}, 63 \%)$ as a pale yellow-green powdery solid. m.p. $98-100{ }^{\circ} \mathrm{C}$. TLC $R_{\mathrm{f}}=0.39\left(5 \% \mathrm{MeOH} / \mathrm{CH}_{2} \mathrm{Cl}_{2}\right)$. IR $v_{\max }$ (neat) $/ \mathrm{cm}^{-1}: 2937 \mathrm{w}$ (C-H str), $2838 \mathrm{w}$ (C-H str), 1623s (C=O str), 1594s (C=C str), 1576s (C=C str), 1512s (C=C str), 1494s, 1463m, 1442m, 1416m, 1247s, 1196m, 1178m, 1094s, 1027m. ${ }^{1} \mathrm{H}-\mathrm{NMR}\left(500 \mathrm{MHz}, \mathrm{CDCl}_{3}\right): \delta 3.83\left(3 \mathrm{H}, \mathrm{s},-\mathrm{OCH}_{3}\right), 3.89$ $\left(3 \mathrm{H}, \mathrm{s},-\mathrm{OCH}_{3}\right), 3.91\left(3 \mathrm{H}, \mathrm{s},-\mathrm{OCH}_{3}\right), 3.93\left(3 \mathrm{H}, \mathrm{s},-\mathrm{OCH}_{3}\right), 3.95\left(3 \mathrm{H}, \mathrm{s},-\mathrm{OCH}_{3}\right), 4.47(2 \mathrm{H}, \mathrm{t}, J=4.8 \mathrm{~Hz}$, $\left.-\mathrm{OCH}_{2} \mathrm{CH}_{2} \mathrm{~N}-\right), 4.86\left(2 \mathrm{H}, \mathrm{t}, J=4.8 \mathrm{~Hz},-\mathrm{OCH}_{2} \mathrm{CH}_{2} \mathrm{~N}-\right), 5.32\left(2 \mathrm{H}, \mathrm{s},-\mathrm{OCH}_{2} \mathrm{CN}-\right), 6.72(1 \mathrm{H}, \mathrm{d}, J=8.4 \mathrm{~Hz}$, $\mathrm{ArH}), 6.85(1 \mathrm{H}, \mathrm{d}, J=8.4 \mathrm{~Hz}, \mathrm{ArH}), 6.95(2 \mathrm{H}, \mathrm{d}, J=8.8 \mathrm{~Hz}, \mathrm{ArH}), 7.01(1 \mathrm{H}, \mathrm{d}, J=2.0 \mathrm{~Hz}, \mathrm{ArH}), 7.04$ $(1 \mathrm{H}, \mathrm{dd}, J=8.8,2.0 \mathrm{~Hz}, \mathrm{ArH}), 7.38(1 \mathrm{H}, \mathrm{d}, J=8.8 \mathrm{~Hz}, \mathrm{ArH}), 7.49(2 \mathrm{H}, \mathrm{d}, J=8.8 \mathrm{~Hz}, \mathrm{ArH}), 7.53(1 \mathrm{H}, \mathrm{d}$, $J=15.6 \mathrm{~Hz},-\mathrm{CH}=\mathrm{CHCO}-), 7.60(1 \mathrm{H}, \mathrm{d}, J=8.4,1.6 \mathrm{~Hz}, \mathrm{ArH}), 7.62(1 \mathrm{H}, \mathrm{d}, J=0.8 \mathrm{~Hz}, \mathrm{ArH}), 7.90(1 \mathrm{H}$, $\mathrm{s},-\mathrm{C}=\mathrm{CH}), 7.98(1 \mathrm{H}, \mathrm{d}, J=16.0 \mathrm{~Hz},-\mathrm{CH}=\mathrm{CHCO}-), 8.06(1 \mathrm{H}, \mathrm{s},-\mathrm{CHN}-), 8.20(1 \mathrm{H}, \mathrm{d}, J=9.2 \mathrm{~Hz}, \mathrm{ArH})$. ${ }^{13} \mathrm{C}-\mathrm{NMR}\left(500 \mathrm{MHz}, \mathrm{CDCl}_{3}\right): \delta 49.8,55.3,56.0,56.0,60.9,61.4,62.4,67.5,101.2,107.6,111.5,112.4$, 113.9, 114.8, 118.7, 120.7, 122.0, 122.4, 123.9, 124.1, 124.7, 124.8, 127.9, 130.1, 133.0, 139.7, 142.4, 143.1, 149.6, 151.0, 152.1, 153.7, 155.7, 157.7, 159.5, 162.4, 175.7, 188.9. LCMS (ES+) $m / z=720.3\left([\mathrm{M}+\mathrm{H}]^{+}\right.$, $t_{\mathrm{R}}=1.84 \mathrm{~min}$ ). HRMS (ESI+) $\mathrm{m} / z=720.2533[\mathrm{M}+\mathrm{H}]^{+}$found, $\mathrm{C}_{40} \mathrm{H}_{38} \mathrm{O}_{10} \mathrm{~N}_{3}{ }^{+}$required 720.2552.

3-Hydroxy-2-(4-(2-(4-(((3-(4-methoxyphenyl)-4-oxo-4H-chromen-7-yl)oxy)methyl)-1H-1,2,3-triazol-1-yl)-etho xy)phenyl)-4H-chromen-4-one (115). A mixture of alkyne isoflavone 46 (183 $\mathrm{mg}, 0.597 \mathrm{mmol})$, azide flavonol 79 (189 mg, $0.585 \mathrm{mmol}), \mathrm{CuSO}_{4} \cdot 5 \mathrm{H}_{2} \mathrm{O}(271 \mathrm{mg}, 1.09 \mathrm{mmol})$ and sodium ascorbate $(493 \mathrm{mg}$, $2.49 \mathrm{mmol})$ in $t-\mathrm{BuOH} / \mathrm{H}_{2} \mathrm{O}(1: 1,40 \mathrm{~mL})$ was reacted according to GP-A. The crude residue was purified by flash column chromatography $\left(\mathrm{SiO}_{2}, 1 \%-5 \% \mathrm{MeOH} / \mathrm{CH}_{2} \mathrm{Cl}_{2}\right)$ and recrystallized from $\mathrm{MeOH}$ to afford triazole hybrid $115(42.9 \mathrm{mg}, 12 \%)$ as a yellow-green powdery solid. m.p. $252-254{ }^{\circ} \mathrm{C}$. TLC $R_{\mathrm{f}}=0.30\left(3 \% \mathrm{MeOH} / \mathrm{CH}_{2} \mathrm{Cl}_{2}\right)$. IR $v_{\max }$ (neat) $/ \mathrm{cm}^{-1}: 3371 \mathrm{w}(\mathrm{br})(\mathrm{O}-\mathrm{H} \mathrm{str}), 3064 \mathrm{w}(\mathrm{C}-\mathrm{H} \mathrm{str})$, 2928w (C-H str), 1640m, 1626m (C=O str), 1601s, 1563m (C=C str), 1512m (C=C str), 1484w, 1444m, 1409m, 1290w, 1247s, 1201m, 1183m, 1121m, 1049m, 1027m. ${ }^{1} \mathrm{H}-\mathrm{NMR}\left(500 \mathrm{MHz}, \mathrm{CDCl}_{3}\right): \delta 3.83(3 \mathrm{H}$, $\left.\mathrm{s},-\mathrm{OCH}_{3}\right), 4.48\left(2 \mathrm{H}, \mathrm{t}, J=5.0 \mathrm{~Hz},-\mathrm{OCH}_{2} \mathrm{CH}_{2} \mathrm{~N}-\right), 4.86\left(2 \mathrm{H}, \mathrm{t}, J=5.0 \mathrm{~Hz},-\mathrm{OCH}_{2} \mathrm{CH}_{2} \mathrm{~N}-\right), 5.36(2 \mathrm{H}, \mathrm{s}$, $\left.-\mathrm{OCH}_{2} \mathrm{CN}-\right), 6.92(2 \mathrm{H}, \mathrm{d}, J=9.0 \mathrm{~Hz}, \mathrm{ArH}), 6.97(3 \mathrm{H}, \mathrm{d}, J=9.0 \mathrm{~Hz}, \mathrm{ArH}$ and OH$), 7.00(1 \mathrm{H}, \mathrm{d}, J=1.5 \mathrm{~Hz}$, ArH), $7.07(1 \mathrm{H}, \mathrm{dd}, J=8.5,2.0 \mathrm{~Hz}, \mathrm{ArH}), 7.42(1 \mathrm{H}, \mathrm{t}, J=7.5 \mathrm{~Hz}, \mathrm{ArH}), 7.47(2 \mathrm{H}, \mathrm{d}, J=8.5 \mathrm{~Hz}, \mathrm{ArH}), 7.57$ $(1 \mathrm{H}, \mathrm{d}, J=8.0 \mathrm{~Hz}, \mathrm{ArH}), 7.70(1 \mathrm{H}, \mathrm{t}, J=8.5 \mathrm{~Hz}, \mathrm{ArH}), 7.89(2 \mathrm{H}, \mathrm{s},-\mathrm{C}=\mathrm{CH}$ and $-\mathrm{CHN}-), 8.21-8.25(4 \mathrm{H}, \mathrm{m}$, ArH). ${ }^{13} \mathrm{C}-\mathrm{NMR}\left(500 \mathrm{MHz}, \mathrm{CDCl}_{3}\right): \delta 49.8,55.3,62.4,66.3,101.3,113.9,114.5,115.0,118.2,118.8,120.6$, $124.1,124.5,124.7,124.9,125.4,127.9,129.6,130.0,133.5,137.8,144.6,152.1,155.3,157.7,159.0,159.5$, $162.3,173.2,175.8$. LCMS (ES+) $m / z=630.2\left([\mathrm{M}+\mathrm{H}]^{+}, t_{\mathrm{R}}=1.77 \mathrm{~min}\right.$ ). HRMS (ESI+) $\mathrm{m} / z=630.1861$ $[\mathrm{M}+\mathrm{H}]^{+}$found, $\mathrm{C}_{36} \mathrm{H}_{28} \mathrm{O}_{8} \mathrm{~N}_{3}{ }^{+}$required 630.1871.

2-((Z)-4-(2-(4-((2-Methoxy-4-((E)-3-(4-methoxyphenyl)acryloyl)phenoxy)methyl)-1H-1,2,3-triazol-1-yl)-etho xy)benzylidene)benzofuran-3(2H)-one (116). A mixture of alkyne chalcone 20 (192 $\mathrm{mg}, 0.595 \mathrm{mmol})$, azide aurone 88 (187 mg, $0.609 \mathrm{mmol}), \mathrm{CuSO}_{4} \cdot 5 \mathrm{H}_{2} \mathrm{O}(289 \mathrm{mg}, 1.16 \mathrm{mmol})$ and sodium ascorbate $(548 \mathrm{mg}$, $2.77 \mathrm{mmol})$ in $t-\mathrm{BuOH} / \mathrm{H}_{2} \mathrm{O}(1: 1,40 \mathrm{~mL})$ was reacted according to GP-A. The crude residue was purified by flash column chromatography $\left(\mathrm{SiO}_{2}, 1 \%-5 \% \mathrm{MeOH} / \mathrm{CH}_{2} \mathrm{Cl}_{2}\right)$ and recrystallized from $\mathrm{MeOH}$ to afford triazole hybrid $\mathbf{1 1 6}(148 \mathrm{mg}, 40 \%)$ as a bright yellow powdery solid. m.p. $128-130{ }^{\circ} \mathrm{C}$. 
TLC $R_{\mathrm{f}}=0.33\left(3 \% \mathrm{MeOH} / \mathrm{CH}_{2} \mathrm{Cl}_{2}\right)$. IR $v_{\max }$ (neat) $/ \mathrm{cm}^{-1}: 2941 \mathrm{w}(\mathrm{C}-\mathrm{H}$ str), $2835 \mathrm{w}$ (C-H str), $1695 \mathrm{~m}$ (C=O str), 1647m, 1590s ( $\mathrm{C}=\mathrm{C}$ str), 1571s (C=C str), 1509s (C=C str), 1461m, 1422m, 1296m, 1250s, 1174s, 1147s, 1128s, 1110s, 1098m, 1025s. ${ }^{1} \mathrm{H}-\mathrm{NMR}\left(500 \mathrm{MHz}, \mathrm{CDCl}_{3}\right): \delta 3.85\left(3 \mathrm{H}, \mathrm{s},-\mathrm{OCH}_{3}\right), 3.97(3 \mathrm{H}$, $\left.\mathrm{s},-\mathrm{OCH}_{3}\right), 4.43\left(2 \mathrm{H}, \mathrm{t}, J=5.0 \mathrm{~Hz},-\mathrm{OCH}_{2} \mathrm{CH}_{2} \mathrm{~N}-\right), 4.80\left(2 \mathrm{H}, \mathrm{t}, J=5.0 \mathrm{~Hz},-\mathrm{OCH}_{2} \mathrm{CH}_{2} \mathrm{~N}-\right), 5.42(2 \mathrm{H}, \mathrm{s}$, $\left.-\mathrm{OCH}_{2} \mathrm{CN}-\right), 6.83(1 \mathrm{H}, \mathrm{s},-\mathrm{C}=\mathrm{CH}), 6.89(2 \mathrm{H}, \mathrm{d}, J=9.0 \mathrm{~Hz}, \mathrm{ArH}), 6.91(2 \mathrm{H}, \mathrm{d}, J=9.0 \mathrm{~Hz}, \mathrm{ArH}), 7.12(1 \mathrm{H}$, $\mathrm{d}, J=8.0 \mathrm{~Hz}, \mathrm{ArH}), 7.22(1 \mathrm{H}, \mathrm{t}, J=8.0 \mathrm{~Hz}, \mathrm{ArH}), 7.33(1 \mathrm{H}, \mathrm{d}, J=8.0 \mathrm{~Hz}, \mathrm{ArH}), 7.39(1 \mathrm{H}, \mathrm{d}, J=15.5 \mathrm{~Hz}$, - $\mathrm{CH}=\mathrm{CHCO}-), 7.56(2 \mathrm{H}, \mathrm{d}, J=8.5 \mathrm{~Hz}, \mathrm{ArH}), 7.60(1 \mathrm{H}, \mathrm{dd}, J=8.5,2.0 \mathrm{~Hz}, \mathrm{ArH}), 7.64-7.67(2 \mathrm{H}, \mathrm{m}, \mathrm{ArH})$, $7.77(1 \mathrm{H}, \mathrm{d}, J=16.0 \mathrm{~Hz},-\mathrm{CH}=\mathrm{CHCO}-), 7.79-7.81(1 \mathrm{H}, \mathrm{m}, \mathrm{ArH}), 7.86(2 \mathrm{H}, \mathrm{d}, J=9.0 \mathrm{~Hz}, \mathrm{ArH}), 7.87(1 \mathrm{H}$, br s, $-\mathrm{CHN}-) .{ }^{13} \mathrm{C}-\mathrm{NMR}\left(500 \mathrm{MHz}, \mathrm{CDCl}_{3}\right): \delta 49.7,55.4,56.1,62.9,66.3,111.1,112.3,112.6,112.9,114.3$, 114.9, 119.1, 121.8, 122.6, 123.3, 124.3, 124.6, 126.1, 127.7, 130.1, 132.2, 133.1, 133.4, 136.6, 144.0, 146.1, $149.6,151.4,158.9,161.5,165.9,184.5,188.6$. LCMS (ES+) $m / z=630.0\left([\mathrm{M}+\mathrm{H}]^{+}, t_{\mathrm{R}}=1.72 \mathrm{~min}\right)$. HRMS (ESI+) $m / z=630.2217[\mathrm{M}+\mathrm{H}]^{+}$found, $\mathrm{C}_{37} \mathrm{H}_{32} \mathrm{O}_{7} \mathrm{~N}_{3}{ }^{+}$required 630.2235 .

2-((Z)-4-(2-(4-((2-((E)-3-(Ferrocenyl)acryloyl)phenoxy)methyl)-1H-1,2,3-triazol-1-yl)ethoxy)benzylidene)benzo furan-3(2H)-one (117). A mixture of alkyne chalcone 26 (286 mg, $0.772 \mathrm{mmol})$, azide aurone 88 (248 $\mathrm{mg}$, $0.806 \mathrm{mmol}), \mathrm{CuSO}_{4} \cdot 5 \mathrm{H}_{2} \mathrm{O}(236 \mathrm{mg}, 0.943 \mathrm{mmol})$ and sodium ascorbate $(426 \mathrm{mg}, 2.15 \mathrm{mmol})$ in $t$ - $\mathrm{BuOH} / \mathrm{H}_{2} \mathrm{O}(1: 1,40 \mathrm{~mL})$ was reacted according to GP-A. The crude residue was purified by flash column chromatography $\left(\mathrm{SiO}_{2}, 1 \%-5 \% \mathrm{MeOH} / \mathrm{CH}_{2} \mathrm{Cl}_{2}\right)$ to afford triazole hybrid $117(409 \mathrm{mg}, 78 \%)$ as a dark red-purple microcrystalline solid. m.p. $108-110{ }^{\circ} \mathrm{C}$. TLC $R_{\mathrm{f}}=0.48\left(3 \% \mathrm{MeOH} / \mathrm{CH}_{2} \mathrm{Cl}_{2}\right)$. IR $v_{\max }$ (neat) $/ \mathrm{cm}^{-1}$ : 3090w (C-H str), 1699m (C=O str), 1646m, 1594s (C=C str), 1569m (C=C str), $1509 \mathrm{~s}$ (C=C str), 1476w, 1459m, 1347w, 1297m, 1247m, 1208m, 1178s, 1127s, 1109s, 1097s, 1044m, 1025m, 1001w. ${ }^{1} \mathrm{H}-\mathrm{NMR}\left(500 \mathrm{MHz}, \mathrm{CDCl}_{3}\right): \delta 4.11\left(5 \mathrm{H}, \mathrm{s},-\mathrm{C}_{5} \mathrm{H}_{5}\right), 4.32\left(2 \mathrm{H}, \mathrm{t}, J=5.2 \mathrm{~Hz},-\mathrm{OCH}_{2} \mathrm{CH}_{2} \mathrm{~N}-\right), 4.42(2 \mathrm{H}, \mathrm{t}$, $\left.J=2.0 \mathrm{~Hz},-\mathrm{C}_{5} \mathrm{H}_{4}\right), 4.47\left(2 \mathrm{H}, \mathrm{t}, J=2.0 \mathrm{~Hz},-\mathrm{C}_{5} \mathrm{H}_{4}\right), 4.65\left(2 \mathrm{H}, \mathrm{t}, J=5.2 \mathrm{~Hz},-\mathrm{OCH}_{2} \mathrm{CH}_{2} \mathrm{~N}-\right), 5.32(2 \mathrm{H}, \mathrm{s}$, $\left.-\mathrm{OCH}_{2} \mathrm{CN}-\right), 6.85(1 \mathrm{H}, \mathrm{s},-\mathrm{C}=\mathrm{CH}), 6.88(2 \mathrm{H}, \mathrm{d}, J=8.8 \mathrm{~Hz}, \mathrm{ArH}), 6.95(1 \mathrm{H}, \mathrm{d}, J=15.6 \mathrm{~Hz},-\mathrm{CH}=\mathrm{CHCO}-)$, $7.08(1 \mathrm{H}, \mathrm{t}, J=7.6 \mathrm{~Hz}, \mathrm{ArH}), 7.13(1 \mathrm{H}, \mathrm{d}, J=8.4 \mathrm{~Hz}, \operatorname{ArH}), 7.22(1 \mathrm{H}, \mathrm{t}, J=7.6 \mathrm{~Hz}, \operatorname{ArH}), 7.34(1 \mathrm{H}, \mathrm{d}$, $J=8.4 \mathrm{~Hz}, \mathrm{ArH}), 7.44-7.46(1 \mathrm{H}, \mathrm{m}, \mathrm{ArH}), 7.49(1 \mathrm{H}, \mathrm{d}, J=15.6 \mathrm{~Hz},-\mathrm{CH}=\mathrm{CHCO}-), 7.60(1 \mathrm{H}, \mathrm{dd}, J=7.6$, $1.6 \mathrm{~Hz}, \mathrm{ArH}), 7.65(1 \mathrm{H}, \mathrm{t}, J=8.4 \mathrm{~Hz}, \mathrm{ArH}), 7.74(1 \mathrm{H}, \mathrm{s},-\mathrm{CHN}-), 7.81(1 \mathrm{H}, \mathrm{dd}, J=7.6,0.8 \mathrm{~Hz}, \mathrm{ArH}), 7.86$ $(2 \mathrm{H}, \mathrm{d}, J=8.8 \mathrm{~Hz}, \mathrm{ArH}) .{ }^{13} \mathrm{C}-\mathrm{NMR}\left(500 \mathrm{MHz}, \mathrm{CDCl}_{3}\right): \delta 49.5,62.9,66.1,68.9,69.8,71.2,79.0,112.8$, 112.9, 113.1, 115.0, 121.4, 121.8, 123.3, 123.9, 124.6, 124.7, 125.9, 130.1, 130.2, 132.4, 133.4, 136.6, 144.1, $145.4,146.0,156.3,159.0,165.8,184.5,192.3$. LCMS (ES +$) m / z=678.2\left([\mathrm{M}+\mathrm{H}]^{+}, t_{\mathrm{R}}=2.11 \mathrm{~min}\right)$. HRMS $(\mathrm{ESI}+) \mathrm{m} / z=678.1677[\mathrm{M}+\mathrm{H}]^{+}$found, $\mathrm{C}_{39} \mathrm{H}_{32} \mathrm{~N}_{3} \mathrm{O}_{5} \mathrm{Fe}^{+}$required 678.1691 .

6-Methoxy-2-((Z)-4-(2-(4-((2-methoxy-5-((E)-3-(1-methyl-1H-pyrrol-2-yl)acryloyl)phenoxy)methyl)-1H-1,2,3triazol-1-yl)ethoxy)benzylidene)benzofuran-3(2H)-one (118). A mixture of alkyne chalcone 22 (265 mg, $0.898 \mathrm{mmol})$, azide aurone $89(304 \mathrm{mg}, 0.900 \mathrm{mmol}), \mathrm{CuSO}_{4} \cdot 5 \mathrm{H}_{2} \mathrm{O}(264 \mathrm{mg}, 1.06 \mathrm{mmol})$ and sodium ascorbate $(474 \mathrm{mg}, 2.39 \mathrm{mmol})$ in $t-\mathrm{BuOH} / \mathrm{H}_{2} \mathrm{O}(1: 1,40 \mathrm{~mL})$ was reacted according to GP-A. The crude residue was purified by flash column chromatography $\left(\mathrm{SiO}_{2}, 1 \%-5 \% \mathrm{MeOH} / \mathrm{CH}_{2} \mathrm{Cl}_{2}\right)$ to afford triazole hybrid $118(270 \mathrm{mg}, 48 \%)$ as a bright yellow powdery solid. m.p. $138-140{ }^{\circ} \mathrm{C}$. TLC $R_{\mathrm{f}}=0.46$ (5\% MeOH $/ \mathrm{CH}_{2} \mathrm{Cl}_{2}$ ). IR $v_{\max }$ (neat) $/ \mathrm{cm}^{-1}: 2934 \mathrm{w}$ (C-H str), $2884 \mathrm{w}$ (C-H str), $1700 \mathrm{~m}$ (C=O str), $1656 \mathrm{~m}, 1638 \mathrm{~m}, 1596 \mathrm{~s}$ (C=C str), 1562s (C=C str), 1510m (C=C str), 1483m, 1412m, 1330m, 1267s, 1198m, 1152s, 1132s, 1099s, 1056m, 1042s, 1022s. ${ }^{1} \mathrm{H}-\mathrm{NMR}\left(500 \mathrm{MHz}, \mathrm{CDCl}_{3}\right): \delta 3.74\left(3 \mathrm{H}, \mathrm{s},-\mathrm{NCH}_{3}\right), 3.94$ $\left(3 \mathrm{H}, \mathrm{s},-\mathrm{OCH}_{3}\right), 3.96\left(3 \mathrm{H}, \mathrm{s},-\mathrm{OCH}_{3}\right), 4.42\left(2 \mathrm{H}, \mathrm{t}, J=4.8 \mathrm{~Hz},-\mathrm{OCH}_{2} \mathrm{CH}_{2} \mathrm{~N}-\right), 4.79(2 \mathrm{H}, \mathrm{t}, J=4.8 \mathrm{~Hz}$, $\left.-\mathrm{OCH}_{2} \mathrm{CH}_{2} \mathrm{~N}-\right), 5.41\left(2 \mathrm{H}, \mathrm{s},-\mathrm{OCH}_{2} \mathrm{CN}-\right), 6.19-6.21(1 \mathrm{H}, \mathrm{m}, \mathrm{ArH}), 6.74-6.80(4 \mathrm{H}, \mathrm{m}, \mathrm{ArH}), 6.78(1 \mathrm{H}$, $\mathrm{s},-\mathrm{C}=\mathrm{CH}), 6.87(2 \mathrm{H}, \mathrm{d}, J=8.8 \mathrm{~Hz}, \mathrm{ArH}), 7.11(1 \mathrm{H}, \mathrm{d}, J=8.4 \mathrm{~Hz}, \operatorname{ArH}), 7.27(1 \mathrm{H}, \mathrm{d}, J=15.2 \mathrm{~Hz}$, - $\mathrm{CH}=\mathrm{CHCO}-$, overlain by $\left.\mathrm{CDCl}_{3}\right), 7.58(1 \mathrm{H}, \mathrm{dd}, J=8.4,1.6 \mathrm{~Hz}, \mathrm{ArH}), 7.64(1 \mathrm{H}, \mathrm{d}, J=2.0 \mathrm{~Hz}, \mathrm{ArH}), 7.69$ $(1 \mathrm{H}, \mathrm{d}, J=8.8 \mathrm{~Hz}, \mathrm{ArH}), 7.78(1 \mathrm{H}, \mathrm{d}, J=15.2 \mathrm{~Hz},-\mathrm{CH}=\mathrm{CHCO}-), 7.82(2 \mathrm{H}, \mathrm{d}, J=8.8 \mathrm{~Hz}, \mathrm{ArH}), 7.87(1 \mathrm{H}$, s, -CHN-). ${ }^{13} \mathrm{C}-\mathrm{NMR}\left(500 \mathrm{MHz}, \mathrm{CDCl}_{3}\right): \delta 34.3,49.7,56.0,56.0,62.8,66.2,96.6,109.6,111.0,111.4,112.0$, $112.1,112.3,114.8,114.9,116.2,122.3,124.3,125.6,126.1,127.6,130.2,131.6,132.4,133.1,143.8,147.0$, 149.4, 151.2, 158.6, 167.2, 168.2, 182.8, 188.0. LCMS (ES+) $m / z=633.3\left([\mathrm{M}+\mathrm{H}]^{+}, t_{\mathrm{R}}=1.94 \mathrm{~min}\right)$. HRMS (ESI+) $m / z=633.2352[\mathrm{M}+\mathrm{H}]^{+}$found, $\mathrm{C}_{36} \mathrm{H}_{33} \mathrm{~N}_{4} \mathrm{O}_{7}{ }^{+}$required 633.2349 . 
2-((Z)-4-(2-(4-((4-Bromo-2-((E)-3-(1-methyl-1H-indol-3-yl)acryloyl)phenoxy)methyl)-1H-1,2,3-triazol-1-yl)eth oxy)benzylidene)benzofuran-3(2H)-one (119). A mixture of alkyne chalcone 21 (308 mg, $0.781 \mathrm{mmol}$ ), azide aurone $88(239 \mathrm{mg}, 0.779 \mathrm{mmol}), \mathrm{CuSO}_{4} \cdot 5 \mathrm{H}_{2} \mathrm{O}(261 \mathrm{mg}, 1.04 \mathrm{mmol})$ and sodium ascorbate $(397 \mathrm{mg}, 2.00 \mathrm{mmol})$ in $t-\mathrm{BuOH} / \mathrm{H}_{2} \mathrm{O}(1: 1,40 \mathrm{~mL})$ was reacted according to GP-A. The crude residue was purified by flash column chromatography $\left(\mathrm{SiO}_{2}, 1 \%-5 \% \mathrm{MeOH} / \mathrm{CH}_{2} \mathrm{Cl}_{2}\right)$ and recrystallized from $\mathrm{MeOH}$ to afford triazole hybrid $119(117 \mathrm{mg}, 21 \%)$ as a pale yellow-brown powdery solid. m.p. $138-140{ }^{\circ} \mathrm{C}$. TLC $R_{\mathrm{f}}=0.44\left(5 \% \mathrm{MeOH} / \mathrm{CH}_{2} \mathrm{Cl}_{2}\right)$. IR $v_{\max }$ (neat) $/ \mathrm{cm}^{-1}$ : 2930w (C-H str), $1699 \mathrm{~m}$ (C=O str), 1644m, 1590s (C=C str), 1525m (C=C str), 1508m (C=C str), 1460m, 1395w, 1373m, 1249m, 1177s, 1126m, 1096w, 1045w. ${ }^{1} \mathrm{H}-\mathrm{NMR}\left(500 \mathrm{MHz} \mathrm{CDCl}_{3}\right): \delta 3.74\left(3 \mathrm{H}, \mathrm{s},-\mathrm{NCH}_{3}\right), 4.03(2 \mathrm{H}, \mathrm{t}, J=5.2 \mathrm{~Hz}$, $\left.-\mathrm{OCH}_{2} \mathrm{CH}_{2} \mathrm{~N}-\right), 4.37\left(2 \mathrm{H}, \mathrm{t}, \mathrm{J}=5.2 \mathrm{~Hz},-\mathrm{OCH}_{2} \mathrm{CH}_{2} \mathrm{~N}-\right), 5.32\left(2 \mathrm{H}, \mathrm{s},-\mathrm{OCH}_{2} \mathrm{CN}-\right), 6.71(2 \mathrm{H}, \mathrm{d}, \mathrm{J}=8.8 \mathrm{~Hz}$, $\mathrm{ArH}), 6.82(1 \mathrm{H}, \mathrm{s},-\mathrm{C}=\mathrm{CH}), 7.04(1 \mathrm{H}, \mathrm{d}, J=8.8 \mathrm{~Hz}, \mathrm{ArH}), 7.16-7.21(1 \mathrm{H}, \mathrm{m}, \mathrm{ArH}), 7.23(1 \mathrm{H}, \mathrm{t}, J=7.6 \mathrm{~Hz}$, ArH), 7.29-7.30 (2H, m, ArH), 7.33-7.35 (2H, m, ArH), 7.38 (1H, d, J = 16.0 Hz, -CH=CHCO-), 7.54 $(1 \mathrm{H}, \mathrm{dd}, J=8.8,2.4 \mathrm{~Hz}, \mathrm{ArH}), 7.64-7.69(2 \mathrm{H}, \mathrm{m}, \mathrm{ArH}), 7.73(1 \mathrm{H}, \mathrm{s},-\mathrm{CHN}-), 7.77(2 \mathrm{H}, \mathrm{d}, J=8.8 \mathrm{ArH})$, 7.80-7.82 (2H, m, ArH), $7.85(1 \mathrm{H}, \mathrm{d}, J=15.6 \mathrm{~Hz},-\mathrm{CH}=\mathrm{CHCO}-) .{ }^{13} \mathrm{C}-\mathrm{NMR}\left(500 \mathrm{MHz}, \mathrm{CDCl}_{3}\right): \delta 33.2$, 49.2, 63.3, 65.7, 110.0, 112.7, 112.7, 112.9, 114.0, 114.7, 114.9, 120.6, 121.7, 121.7, 121.8, 123.1, 123.4, 124.1, 124.6, 125.7, 125.8, 132.0, 133.1, 133.2, 134.8, 135.0, 136.7, 138.1, 138.1, 143.7, 146.0, 155.5, 158.8, 165.8, 184.5, 190.8. LCMS (ES+) $m / z=703.1\left([\mathrm{M}+\mathrm{H}]^{+}, t_{\mathrm{R}}=5.05 \mathrm{~min}\right)$. HRMS (ESI+) $m / z=723.1189$ $[\mathrm{M}+\mathrm{Na}]^{+}$found, $\mathrm{C}_{38} \mathrm{H}_{29} \mathrm{O}_{5} \mathrm{~N}_{4} \mathrm{BrNa}^{+}$required 723.1214 .

(Z)-3-((1-(2-(4-((6-Methoxy-3-oxobenzofuran-2(3H)-ylidene)methyl)phenoxy)ethyl)-1H-1,2,3-triazol-4-yl)-meth oxy)-2-phenyl-4H-chromen-4-one (120). A mixture of alkyne flavone 30 (250 mg, $0.906 \mathrm{mmol})$, azide aurone 89 (316 mg, $0.936 \mathrm{mmol}), \mathrm{CuSO}_{4} \cdot 5 \mathrm{H}_{2} \mathrm{O}(284 \mathrm{mg}, 1.14 \mathrm{mmol})$ and sodium ascorbate $(447 \mathrm{mg}$, $2.26 \mathrm{mmol})$ in $t-\mathrm{BuOH} / \mathrm{H}_{2} \mathrm{O}(1: 1,40 \mathrm{~mL})$ was reacted according to GP-A. The crude residue was purified by flash column chromatography $\left(\mathrm{SiO}_{2}, 1 \%-5 \% \mathrm{MeOH} / \mathrm{CH}_{2} \mathrm{Cl}_{2}\right)$ and recrystallized from $\mathrm{MeOH}$ to afford triazole hybrid $120(351 \mathrm{mg}, 63 \%)$ as a pale yellow-white powdery solid. m.p. $186-188^{\circ} \mathrm{C}$. TLC $R_{\mathrm{f}}=0.38\left(5 \% \mathrm{MeOH} / \mathrm{CH}_{2} \mathrm{Cl}_{2}\right)$. IR $v_{\max }$ (neat) $/ \mathrm{cm}^{-1}$ : 3056w (C-H str), $1698 \mathrm{~m}$ (C=O str), 1654w, 1608s, 1595s (C=C str), 1509m (C=C str), 1470m, 1434m, 1397m, 1342m, 1272s, 1245s, 1198s, 1176m, 1147m, 1128s, 1109s, 1091s, 1042m. ${ }^{1} \mathrm{H}-\mathrm{NMR}\left(500 \mathrm{MHz}, \mathrm{CDCl}_{3}\right): \delta 3.93\left(3 \mathrm{H}, \mathrm{s},-\mathrm{OCH}_{3}\right)$, $4.35\left(2 \mathrm{H}, \mathrm{t}, J=5.0 \mathrm{~Hz},-\mathrm{OCH}_{2} \mathrm{CH}_{2} \mathrm{~N}-\right), 4.71\left(2 \mathrm{H}, \mathrm{t}, \mathrm{J}=4.5 \mathrm{~Hz},-\mathrm{OCH}_{2} \mathrm{CH}_{2} \mathrm{~N}-\right), 5.33\left(2 \mathrm{H}, \mathrm{s},-\mathrm{OCH} \mathrm{CN}^{-}\right)$, 6.74-6.77 (3H, m, ArH and $-\mathrm{C}=\mathrm{CH}), 6.90(2 \mathrm{H}, \mathrm{d}, J=9.0 \mathrm{~Hz}, \mathrm{ArH}), 7.40-7.45(4 \mathrm{H}, \mathrm{m}, \mathrm{ArH}), 7.52(1 \mathrm{H}, \mathrm{dd}$, $J=8.5,0.5 \mathrm{~Hz}, \mathrm{ArH}), 7.69(1 \mathrm{H}, \mathrm{t}, J=8.5 \mathrm{~Hz}, \mathrm{ArH}), 7.70(1 \mathrm{H}, \mathrm{d}, J=8.5 \mathrm{~Hz}, \mathrm{ArH}), 7.83(2 \mathrm{H}, \mathrm{d}, J=9.0 \mathrm{~Hz}$, $\mathrm{ArH}), 7.83(1 \mathrm{H}, \mathrm{s},-\mathrm{CHN}-), 8.01-8.03(2 \mathrm{H}, \mathrm{m}, \mathrm{ArH}), 8.28(1 \mathrm{H}, \mathrm{dd}, J=8.0,1.5 \mathrm{~Hz}, \mathrm{ArH}) .{ }^{13} \mathrm{C}-\mathrm{NMR}$ $\left(500 \mathrm{MHz}, \mathrm{CDCl}_{3}\right): \delta 49.5,56.0,65.1,66.2,96.6,111.5,112.0,114.9,115.0,118.1,124.1,124.8,124.9,125.7$, 126.1, 128.3, 128.7, 130.7, 133.1, 133.5, 139.5, 144.0, 147.0, 155.3, 156.4, 158.8, 167.3, 168.3, 175.1, 182.8. LCMS (ES+) $m / z=614.2\left([\mathrm{M}+\mathrm{H}]^{+}, t_{\mathrm{R}}=1.91 \mathrm{~min}\right)$. HRMS (ESI+) $m / z=614.1926[\mathrm{M}+\mathrm{H}]^{+}$found, $\mathrm{C}_{36} \mathrm{H}_{28} \mathrm{~N}_{3} \mathrm{O}_{7}^{+}$required 614.1927 .

(Z)-6-((1-(2-(4-((6-Methoxy-3-oxobenzofuran-2(3H)-ylidene)methyl)phenoxy)ethyl)-1H-1,2,3-triazol-4-yl)meth oxy)-2-phenyl-4H-chromen-4-one (121). A mixture of alkyne flavone 31 (249 $\mathrm{mg}, 0.900 \mathrm{mmol})$, azide aurone $89(309 \mathrm{mg}, 0.916 \mathrm{mmol}), \mathrm{CuSO}_{4} \cdot 5 \mathrm{H}_{2} \mathrm{O}(247 \mathrm{mg}, 0.989 \mathrm{mmol})$ and sodium ascorbate (482 $\mathrm{mg}$, $2.43 \mathrm{mmol})$ in $t-\mathrm{BuOH} / \mathrm{H}_{2} \mathrm{O}(1: 1,40 \mathrm{~mL})$ was reacted according to GP-A. The crude residue was purified by flash column chromatography $\left(\mathrm{SiO}_{2}, 1 \%-5 \% \mathrm{MeOH} / \mathrm{CH}_{2} \mathrm{Cl}_{2}\right)$ and recrystallized from $\mathrm{MeOH}$ to afford triazole hybrid 121 (269 $\mathrm{mg}, 49 \%)$ as a bright yellow-orange powdery solid. m.p. $148-150{ }^{\circ} \mathrm{C}$. TLC $R_{\mathrm{f}}=0.30\left(5 \% \mathrm{MeOH} / \mathrm{CH}_{2} \mathrm{Cl}_{2}\right)$. IR $v_{\max }$ (neat) $/ \mathrm{cm}^{-1}$ : $3065 \mathrm{w}(\mathrm{C}-\mathrm{H}$ str), 2925w (C-H str), 1695m (C=O str), 1640s, 1594s (C=C str), 1568s (C=C str), 1510m (C=C str), 1496m, $1481 \mathrm{~m}, 1454 \mathrm{~s}, 1442 \mathrm{~s}, 1360 \mathrm{~m}, 1270 \mathrm{~m}, 1250 \mathrm{~s}, 1181 \mathrm{~s}, 1131 \mathrm{~s}, 1110 \mathrm{~s}, 1095 \mathrm{~s}, 1043 \mathrm{~m}, 1027 \mathrm{~m} .{ }^{1} \mathrm{H}-\mathrm{NMR}$ $\left(500 \mathrm{MHz} \mathrm{CDCl}_{3}\right): \delta 3.92\left(3 \mathrm{H}, \mathrm{s},-\mathrm{OCH}_{3}\right), 4.43\left(2 \mathrm{H}, \mathrm{t}, \mathrm{J}=4.8 \mathrm{~Hz},-\mathrm{OCH}_{2} \mathrm{CH}_{2} \mathrm{~N}-\right), 4.82(2 \mathrm{H}, \mathrm{t}, J=4.8 \mathrm{~Hz}$, $\left.-\mathrm{OCH}_{2} \mathrm{CH}_{2} \mathrm{~N}-\right), 5.30\left(2 \mathrm{H}, \mathrm{s},-\mathrm{OCH}_{2} \mathrm{CN}-\right), 6.72-6.74(2 \mathrm{H}, \mathrm{m}, \mathrm{ArH}), 6.75(1 \mathrm{H}, \mathrm{s},-\mathrm{C}=\mathrm{CH}), 6.79(1 \mathrm{H}, \mathrm{s}$, $-\mathrm{C}=\mathrm{CH}), 6.89(2 \mathrm{H}, \mathrm{d}, J=8.8 \mathrm{~Hz}, \mathrm{ArH}), 7.33(1 \mathrm{H}, \mathrm{dd}, J=9.2,3.2 \mathrm{~Hz}, \mathrm{ArH}), 7.48-7.52(4 \mathrm{H}, \mathrm{m}, \mathrm{ArH}), 7.68$ $(1 \mathrm{H}, \mathrm{d}, J=9.2 \mathrm{~Hz}, \mathrm{ArH}), 7.71(1 \mathrm{H}, \mathrm{d}, J=3.2 \mathrm{~Hz}, \mathrm{ArH}), 7.80(2 \mathrm{H}, \mathrm{d}, J=8.8 \mathrm{~Hz}, \mathrm{ArH}), 7.88-7.90$ (3H, m, ArH and -CHN-). ${ }^{13} \mathrm{C}-\mathrm{NMR}\left(500 \mathrm{MHz} \mathrm{CDCl}_{3}\right): \delta 49.7,56.0,62.2,66.3,96.5,106.4,106.8,111.4,112.0$, 
$114.8,114.9,120.0,123.9,124.2,124.5,125.7,126.1,126.2,129.0,131.5,131.7,133.1,143.4,146.9,151.2$, $155.4,158.7,163.2,167.2,168.2,178.1,182.8$. LCMS (ES+) $m / z=614.2\left([\mathrm{M}+\mathrm{H}]^{+}, t_{\mathrm{R}}=1.77 \mathrm{~min}\right)$. HRMS $(\mathrm{ESI}+) \mathrm{m} / z=614.1906[\mathrm{M}+\mathrm{H}]^{+}$found, $\mathrm{C}_{36} \mathrm{H}_{28} \mathrm{O}_{7} \mathrm{~N}_{3}{ }^{+}$required 614.1922.

(Z)-3-Hydroxy-2-(4-((1-(2-(4-((3-oxobenzofuran-2(3H)-ylidene)methyl)phenoxy)ethyl)-1H-1,2,3-triazol-4-yl) methoxy)phenyl)-4H-chromen-4-one (122). A mixture of alkyne flavonol 36 (391 mg, $1.34 \mathrm{mmol})$, azide aurone $88(408 \mathrm{mg}, 1.33 \mathrm{mmol}), \mathrm{CuSO}_{4} \cdot 5 \mathrm{H}_{2} \mathrm{O}(435 \mathrm{mg}, 1.74 \mathrm{mmol})$ and sodium ascorbate $(682 \mathrm{mg}$, $3.44 \mathrm{mmol})$ in $t-\mathrm{BuOH} / \mathrm{H}_{2} \mathrm{O}(1: 1,40 \mathrm{~mL})$ was reacted according to GP-A. The crude residue was purified by flash column chromatography $\left(\mathrm{SiO}_{2}, 1 \%-5 \% \mathrm{MeOH} / \mathrm{CH}_{2} \mathrm{Cl}_{2}\right)$ and recrystallized from $\mathrm{MeOH}$ to afford triazole hybrid 122 (335 mg, 42\%) as a yellow-brown powdery solid. m.p. $208-210^{\circ} \mathrm{C}$. TLC $R_{\mathrm{f}}=0.32\left(5 \% \mathrm{MeOH} / \mathrm{CH}_{2} \mathrm{Cl}_{2}\right)$. IR $v_{\max }$ (neat) $/ \mathrm{cm}^{-1}: 3300 \mathrm{w}(\mathrm{O}-\mathrm{H}$ str), 3073w (C-H str), 2951w (C-H str), 1694m (C=O str), 1646w, 1604s, 1596s (C=C str), 1567w (C=C str), 1509s (C=C str), 1458w, 1426w, 1407w, 1299m, 1256s, 1178s, 1109s, 1051s. ${ }^{1} \mathrm{H}-\mathrm{NMR}\left(500 \mathrm{MHz}, \mathrm{CDCl}_{3}\right): \delta 4.46(2 \mathrm{H}, \mathrm{t}, J=4.8 \mathrm{~Hz}$, $\left.-\mathrm{OCH}_{2} \mathrm{CH}_{2} \mathrm{~N}-\right), 4.84\left(2 \mathrm{H}, \mathrm{t}, J=4.8 \mathrm{~Hz},-\mathrm{OCH}_{2} \mathrm{CH}_{2} \mathrm{~N}-\right), 5.34\left(2 \mathrm{H}, \mathrm{s},-\mathrm{OCH}_{2} \mathrm{CN}-\right), 6.82(1 \mathrm{H}, \mathrm{s},-\mathrm{C}=\mathrm{CH})$, $6.92(2 \mathrm{H}, \mathrm{d}, J=8.8 \mathrm{~Hz}, \mathrm{ArH}), 6.96(1 \mathrm{H}, \mathrm{br} \mathrm{s}, \mathrm{OH}), 7.15(2 \mathrm{H}, \mathrm{d}, J=8.8 \mathrm{~Hz}, \mathrm{ArH}), 7.22(1 \mathrm{H}, \mathrm{t}, J=7.6 \mathrm{~Hz}$, ArH), $7.31(1 \mathrm{H}, \mathrm{d}, J=8.4 \mathrm{~Hz}, \mathrm{ArH}), 7.39(1 \mathrm{H}, \mathrm{t}, J=7.6 \mathrm{~Hz}, \mathrm{ArH}), 7.56(1 \mathrm{H}, \mathrm{d}, J=8.8 \mathrm{~Hz}, \operatorname{ArH}), 7.63-7.71$ $(2 \mathrm{H}, \mathrm{m}, \mathrm{ArH}), 7.80(1 \mathrm{H}, \mathrm{d}, J=7.6 \mathrm{~Hz}, \mathrm{ArH}), 7.86(3 \mathrm{H}, \mathrm{d}, J=8.4 \mathrm{~Hz}, \mathrm{ArH}$ and $-\mathrm{CHN}-), 8.21-8.25(3 \mathrm{H}$, $\mathrm{m}, \mathrm{ArH}) .{ }^{13} \mathrm{C}-\mathrm{NMR}\left(500 \mathrm{MHz}, \mathrm{CDCl}_{3}\right): \delta 49.8,62.1,66.4,112.6,112.9,114.9,114.9,118.1,120.6,121.8$, 123.4, 124.1, 124.4, 124.6, 125.4, 126.1, 129.5, 133.0, 133.4, 136.5, 136.7, 137.7, 145.0, 146.1, 155.3, 157.4, $158.9,159.5,165.9,173.1,184.5$. LCMS (ES+) $m / z=600.0\left([\mathrm{M}+\mathrm{H}]^{+}, t_{\mathrm{R}}=4.68 \mathrm{~min}\right)$. HRMS (ESI+) $m / z$ $=600.1751[\mathrm{M}+\mathrm{H}]^{+}$found, $\mathrm{C}_{35} \mathrm{H}_{26} \mathrm{O}_{7} \mathrm{~N}_{3}{ }^{+}$required 600.1765 .

(Z)-2-(4-(2-(4-((3-((4,6-Dimethoxy-3-oxobenzofuran-2(3H)-ylidene)methyl)phenoxy)methyl)-1H-1,2,3-triazol-1yl)ethoxy)phenyl)-3-hydroxy-7-methoxy-4H-chromen-4-one (123). A mixture of alkyne aurone 56 (192 mg, $0.571 \mathrm{mmol})$, azide flavonol $80(210 \mathrm{mg}, 0.595 \mathrm{mmol}), \mathrm{CuSO}_{4} \cdot 5 \mathrm{H}_{2} \mathrm{O}(234 \mathrm{mg}, 0.939 \mathrm{mmol})$ and sodium ascorbate $(362 \mathrm{mg}, 1.83 \mathrm{mmol})$ in $t-\mathrm{BuOH} / \mathrm{H}_{2} \mathrm{O}(1: 1,40 \mathrm{~mL})$ was reacted according to GP-A. The crude residue was purified by flash column chromatography $\left(\mathrm{SiO}_{2}, 1 \%-5 \% \mathrm{MeOH} / \mathrm{CH}_{2} \mathrm{Cl}_{2}\right)$ and recrystallized from $\mathrm{MeOH}$ to afford triazole hybrid 123 (54.3 $\mathrm{mg}, 14 \%$ ) as a pale yellow-brown powdery solid. m.p. $178-180{ }^{\circ} \mathrm{C}$. TLC $R_{\mathrm{f}}=0.29\left(5 \% \mathrm{MeOH} / \mathrm{CH}_{2} \mathrm{Cl}_{2}\right)$. IR $v_{\max }$ (neat) $/ \mathrm{cm}^{-1}: 3302 \mathrm{w}$ (O-H str), 2939w (C-H str), 2845w (C-H str), 1692w, 1614s (C=O str), 1599s (C=C str), 1510m (C=C str), $1503 \mathrm{~m}(\mathrm{C}=\mathrm{C}$ str) $1452 \mathrm{~m}, 1403 \mathrm{w}, 1361 \mathrm{w}, 1346 \mathrm{w}, 1251 \mathrm{~m}, 1215 \mathrm{~s}, 1187 \mathrm{w}, 1156 \mathrm{~m}, 1121 \mathrm{w}, 1092 \mathrm{~s}, 1036 \mathrm{w}$. ${ }^{1} \mathrm{H}-\mathrm{NMR}\left(500 \mathrm{MHz}, \mathrm{CDCl}_{3}\right): \delta 3.91\left(3 \mathrm{H}, \mathrm{s},-\mathrm{OCH}_{3}\right), 3.92\left(3 \mathrm{H}, \mathrm{s},-\mathrm{OCH}_{3}\right), 3.95\left(3 \mathrm{H}, \mathrm{s},-\mathrm{OCH}_{3}\right), 4.46(2 \mathrm{H}, \mathrm{t}$, $\left.J=4.5 \mathrm{~Hz},-\mathrm{OCH}_{2} \mathrm{CH}_{2} \mathrm{~N}-\right), 4.83\left(2 \mathrm{H}, \mathrm{t}, J=4.5 \mathrm{~Hz},-\mathrm{OCH}_{2} \mathrm{CH}_{2} \mathrm{~N}-\right), 5.34\left(2 \mathrm{H}, \mathrm{s},-\mathrm{OCH}_{2} \mathrm{CN}-\right), 6.08(1 \mathrm{H}, \mathrm{s}$, $\mathrm{ArH}), 6.41(1 \mathrm{H}, \mathrm{s}, \mathrm{ArH}), 6.70(1 \mathrm{H}, \mathrm{s},-\mathrm{C}=\mathrm{CH}), 6.93-6.95(4 \mathrm{H}, \mathrm{m}, \mathrm{ArH}$ and $\mathrm{OH}), 6.99-7.03$ (2H, m, ArH), $7.34(1 \mathrm{H}, \mathrm{t}, J=8.0 \mathrm{~Hz}, \mathrm{ArH}), 7.40(1 \mathrm{H}, \mathrm{d}, J=7.5 \mathrm{~Hz}, \mathrm{ArH}), 7.55(1 \mathrm{H}, \mathrm{s}, \mathrm{ArH}), 7.85(1 \mathrm{H}$, br s, $-\mathrm{CHN}-)$, $8.12(1 \mathrm{H}, \mathrm{d}, J=9.0 \mathrm{~Hz}, \mathrm{ArH}), 8.16(2 \mathrm{H}, \mathrm{d}, J=8.5 \mathrm{~Hz}, \mathrm{ArH}) .{ }^{13} \mathrm{C}-\mathrm{NMR}\left(500 \mathrm{MHz}, \mathrm{CDCl}_{3}\right): \delta 49.8,55.9$, 56.2, 56.2, 62.2, 66.4, 89.3, 94.1, 99.9, 105.1, 110.3, 114.4, 114.6, 114.8, 116.2, 116.7, 121.9, 123.9, 124.5, 124.8, 126.7, 129.3, 129.8, 134.0, 137.4, 144.0, 148.0, 157.2, 158.3, 158.8, 159.3, 164.2, 169.1, 172.6, 180.6 . LCMS (ES+) $m / z=690.2\left([\mathrm{M}+\mathrm{H}]^{+}, t_{\mathrm{R}}=4.59 \mathrm{~min}\right)$. HRMS (ESI+) $m / z=690.2062[\mathrm{M}+\mathrm{H}]^{+}$found, $\mathrm{C}_{38} \mathrm{H}_{32} \mathrm{O}_{10} \mathrm{~N}_{3}{ }^{+}$required 690.2082.

(Z)-7-((1-(2-(4-((6-Methoxy-3-oxobenzofuran-2(3H)-ylidene)methyl)phenoxy)ethyl)-1H-1,2,3-triazol-4-yl)-met hoxy)-3-(4-methoxyphenyl)-4H-chromen-4-one (124). A mixture of alkyne isoflavone 46 (276 mg, $0.901 \mathrm{mmol})$, azide aurone $89(301 \mathrm{mg}, 0.892 \mathrm{mmol}), \mathrm{CuSO}_{4} \cdot 5 \mathrm{H}_{2} \mathrm{O}(348 \mathrm{mg}, 1.39 \mathrm{mmol})$ and sodium ascorbate $(496 \mathrm{mg}, 2.50 \mathrm{mmol})$ in $t-\mathrm{BuOH} / \mathrm{H}_{2} \mathrm{O}(1: 1,40 \mathrm{~mL})$ was reacted according to GP-A. The crude residue was purified by flash column chromatography $\left(\mathrm{SiO}_{2}, 1 \%-5 \% \mathrm{MeOH} / \mathrm{CH}_{2} \mathrm{Cl}_{2}\right)$ and recrystallized from $\mathrm{MeOH}$ to afford triazole hybrid $124(317 \mathrm{mg}, 55 \%)$ as a bright yellow powdery solid. m.p. $228-230{ }^{\circ} \mathrm{C}$. TLC $R_{\mathrm{f}}=0.43\left(5 \% \mathrm{MeOH} / \mathrm{CH}_{2} \mathrm{Cl}_{2}\right)$. IR $v_{\max }$ (neat) $/ \mathrm{cm}^{-1}: 3085 \mathrm{w}(\mathrm{C}-\mathrm{H} \mathrm{str})$, 2935w (C-H str), 1705m (C=O str), 1651m, 1629s, 1596s (C=C str), 1567m (C=C str), 1510s (C=C str), 1441s, 1347m, 1295m, 1249s, 1202m, 1179s, 1147s, 1098s, 1020s. ${ }^{1} \mathrm{H}-\mathrm{NMR}\left(500 \mathrm{MHz}, \mathrm{CDCl}_{3}\right): \delta 3.83$ $\left(3 \mathrm{H}, \mathrm{s},-\mathrm{OCH}_{3}\right), 3.93\left(3 \mathrm{H}, \mathrm{s},-\mathrm{OCH}_{3}\right), 4.44\left(2 \mathrm{H}, \mathrm{t}, \mathrm{J}=4.8 \mathrm{~Hz},-\mathrm{OCH}_{2} \mathrm{CH}_{2} \mathrm{~N}-\right), 4.83(2 \mathrm{H}, \mathrm{t}, J=4.8 \mathrm{~Hz}$, $\left.-\mathrm{OCH}_{2} \mathrm{CH}_{2} \mathrm{~N}-\right), 5.34\left(2 \mathrm{H}, \mathrm{s},-\mathrm{OCH}_{2} \mathrm{CN}-\right), 6.74-6.77(2 \mathrm{H}, \mathrm{m}, \mathrm{ArH}), 6.76(1 \mathrm{H}, \mathrm{s},-\mathrm{C}=\mathrm{CH}), 6.88(2 \mathrm{H}, \mathrm{d}$, 
$J=8.8 \mathrm{~Hz}, \mathrm{ArH}), 6.93(2 \mathrm{H}, \mathrm{d}, J=8.8 \mathrm{~Hz}, \mathrm{ArH}), 6.97(1 \mathrm{H}, \mathrm{d}, J=2.4 \mathrm{~Hz}, \mathrm{ArH}), 7.06(1 \mathrm{H}, \mathrm{dd}, J=8.8$, $2.4 \mathrm{~Hz}, \mathrm{ArH}), 7.47(2 \mathrm{H}, \mathrm{d}, J=8.8 \mathrm{~Hz}, \mathrm{ArH}), 7.69(1 \mathrm{H}, \mathrm{d}, J=8.4 \mathrm{~Hz}, \mathrm{ArH}), 7.82(2 \mathrm{H}, \mathrm{d}, J=8.8 \mathrm{~Hz}$, $\mathrm{ArH}), 7.87(1 \mathrm{H}, \mathrm{s},-\mathrm{CHN}-), 7.89(1 \mathrm{H}, \mathrm{s},-\mathrm{C}=\mathrm{CH}), 8.23(1 \mathrm{H}, \mathrm{d}, J=8.8 \mathrm{~Hz}, \mathrm{ArH}) .{ }^{13} \mathrm{C}-\mathrm{NMR}(500 \mathrm{MHz}$, $\left.\mathrm{CDCl}_{3}\right): \delta 49.8,55.3,56.0,62.4,66.3,96.7,101.3,111.3,112.1,113.9,114.8,114.9,118.8,124.1,124.2,124.9$, 125.7, 126.3, 127.9, 130.0, 133.1, 143.2, 147.0, 152.1, 157.7, 158.6, 159.5, 162.3, 167.3, 168.3, 175.8, 182.8 . LCMS (ES+) $m / z=644.2\left([\mathrm{M}+\mathrm{H}]^{+}, t_{\mathrm{R}}=1.86 \mathrm{~min}\right)$. HRMS (ESI+) $m / z=644.2011[\mathrm{M}+\mathrm{H}]^{+}$found, $\mathrm{C}_{37} \mathrm{H}_{30} \mathrm{O}_{8} \mathrm{~N}_{3}{ }^{+}$required 644.2027.

4,6-Dimethoxy-2-((Z)-3-((1-(2-(4-(((Z)-3-oxobenzofuran-2(3H)-ylidene)methyl)phenoxy)ethyl)-1H-1,2,3-triazol -4-yl)methoxy)benzylidene)benzofuran-3(2H)-one (125). A mixture of alkyne aurone 56 (303 mg, $0.902 \mathrm{mmol})$, azide aurone $88(278 \mathrm{mg}, 0.905 \mathrm{mmol}), \mathrm{CuSO}_{4} \cdot 5 \mathrm{H}_{2} \mathrm{O}(263 \mathrm{mg}, 1.05 \mathrm{mmol})$ and sodium ascorbate $(462 \mathrm{mg}, 2.33 \mathrm{mmol})$ in $t-\mathrm{BuOH} / \mathrm{H}_{2} \mathrm{O}(1: 1,20 \mathrm{~mL})$ was reacted according to GP-A. The crude residue was purified by flash column chromatography $\left(\mathrm{SiO}_{2}, 1 \%-5 \% \mathrm{MeOH} / \mathrm{CH}_{2} \mathrm{Cl}_{2}\right)$ and recrystallized from $\mathrm{MeOH}$ to afford triazole hybrid $125(491 \mathrm{mg}, 85 \%)$ as a bright yellow-brown powdery solid. m.p. $148-150{ }^{\circ} \mathrm{C}$. TLC $R_{\mathrm{f}}=0.36\left(5 \% \mathrm{MeOH} / \mathrm{CH}_{2} \mathrm{Cl}_{2}\right)$. IR $v_{\max }$ (neat) $/ \mathrm{cm}^{-1}: 2942 \mathrm{w}$ (C-H str), 2843w (C-H str), 1694m (C=O str), 1651m, 1587s (C=C str), 1508m (C=C str), 1458m, 1423w, 1345m, 1298m, 1251m, 1234m, 1214s, 1177s, 1154s, 1128s, 1091s, 1036m. ${ }^{1} \mathrm{H}-\mathrm{NMR}\left(500 \mathrm{MHz}, \mathrm{CDCl}_{3}\right)$ : $\delta 3.90\left(3 \mathrm{H}, \mathrm{s},-\mathrm{OCH}_{3}\right), 3.92\left(3 \mathrm{H}, \mathrm{s},-\mathrm{OCH}_{3}\right), 4.42\left(2 \mathrm{H}, \mathrm{t}, J=4.8 \mathrm{~Hz},-\mathrm{OCH}_{2} \mathrm{CH}_{2} \mathrm{~N}-\right), 4.81(2 \mathrm{H}, \mathrm{t}, J=4.8 \mathrm{~Hz}$, $\left.-\mathrm{OCH}_{2} \mathrm{CH}_{2} \mathrm{~N}-\right), 5.31\left(2 \mathrm{H}, \mathrm{s},-\mathrm{OCH}_{2} \mathrm{CN}-\right), 6.09(1 \mathrm{H}, \mathrm{d}, J=1.6 \mathrm{~Hz}, \mathrm{ArH}), 6.40(1 \mathrm{H}, \mathrm{d}, J=1.2 \mathrm{~Hz}, \mathrm{ArH})$, $6.68(1 \mathrm{H}, \mathrm{s},-\mathrm{C}=\mathrm{CH}), 6.81(1 \mathrm{H}, \mathrm{s},-\mathrm{C}=\mathrm{CH}), 6.88(2 \mathrm{H}, \mathrm{d}, J=8.4 \mathrm{~Hz}, \mathrm{ArH}), 7.00(1 \mathrm{H}, \mathrm{dd}, J=8.0,2.4 \mathrm{~Hz}$, $\operatorname{ArH}), 7.21(1 \mathrm{H}, \mathrm{t}, J=7.6 \mathrm{~Hz}, \operatorname{ArH}), 7.30-7.34(2 \mathrm{H}, \mathrm{m}, \operatorname{ArH}), 7.39(1 \mathrm{H}, \mathrm{d}, J=8.0 \mathrm{~Hz}, \operatorname{ArH}), 7.54(1 \mathrm{H}, \mathrm{s}$, $\mathrm{ArH}), 7.64(1 \mathrm{H}, \mathrm{t}, J=7.6 \mathrm{~Hz}, \mathrm{ArH}), 7.79(1 \mathrm{H}, \mathrm{d}, J=7.6 \mathrm{~Hz}, \mathrm{ArH}), 7.82-7.85(3 \mathrm{H}, \mathrm{m}, \mathrm{ArH}$ and $-\mathrm{CHN}-$ ). ${ }^{13} \mathrm{C}-\mathrm{NMR}\left(500 \mathrm{MHz}, \mathrm{CDCl}_{3}\right): \delta 49.7,56.2,62.2,66.3,89.3,94.1,105.1,110.3,112.7,112.9,114.3,114.8$, 116.3, 116.6, 121.8, 123.3, 123.9, 124.5, 124.5, 126.0, 129.8, 133.0, 133.4, 133.9, 136.7, 144.3, 146.1, 148.0, 158.3, 158.9, 159.3, 165.9, 169.0, 180.6, 184.5. LCMS (ES+) $m / z=644.2\left([\mathrm{M}+\mathrm{H}]^{+}, t_{\mathrm{R}}=2.02 \mathrm{~min}\right)$. HRMS (ESI+) $m / z=644.2006[\mathrm{M}+\mathrm{H}]^{+}$found, $\mathrm{C}_{37} \mathrm{H}_{30} \mathrm{O}_{8} \mathrm{~N}_{3}{ }^{+}$required 644.2027 .

(E)-4-((1-(2-(4-(3-(2-Hydroxyphenyl)-3-oxoprop-1-en-1-yl)phenoxy)ethyl)-1H-1,2,3-triazol-4-yl)methoxy)-2Hchromen-2-one (126). A mixture of alkyne coumarin 48 (207 mg, $1.03 \mathrm{mmol})$, azide chalcone 61 (302 mg, $0.975 \mathrm{mmol}), \mathrm{CuSO}_{4} \cdot 5 \mathrm{H}_{2} \mathrm{O}(292 \mathrm{mg}, 1.17 \mathrm{mmol})$ and sodium ascorbate (508 $\left.\mathrm{mg}, 2.56 \mathrm{mmol}\right)$ in $t-\mathrm{BuOH} / \mathrm{H}_{2} \mathrm{O}(1: 1,40 \mathrm{~mL})$ was reacted according to GP-A. The crude residue was purified by flash column chromatography $\left(\mathrm{SiO}_{2}, 1 \%-5 \% \mathrm{MeOH} / \mathrm{CH}_{2} \mathrm{Cl}_{2}\right)$ and recrystallized from $\mathrm{MeOH}$ to afford triazole hybrid 126 ( $387 \mathrm{mg}, 78 \%$ ) as a bright yellow powdery solid. m.p. $194-196{ }^{\circ} \mathrm{C}$. TLC $R_{\mathrm{f}}=0.43$ $\left(5 \% \mathrm{MeOH} / \mathrm{CH}_{2} \mathrm{Cl}_{2}\right)$. IR $v_{\max }$ (neat) $/ \mathrm{cm}^{-1}$ : 3083w (C-H str), 2929w (C-H str), 1725s (C=O str), 1641m, $1625 \mathrm{~m}, 1607 \mathrm{~m}, 1560 \mathrm{~s}$ (C=C str), 1511s (C=C str), 1489s, 1424m, 1382m, 1273m, 1249s, 1202s, 1175s, $1156 \mathrm{~s}, 1107 \mathrm{~m}, 1056 \mathrm{~m}, 1030 \mathrm{~m} .{ }^{1} \mathrm{H}-\mathrm{NMR}\left(500 \mathrm{MHz}, \mathrm{CDCl}_{3}\right): \delta 4.47\left(2 \mathrm{H}, \mathrm{t}, J=5.2 \mathrm{~Hz},-\mathrm{OCH}_{2} \mathrm{CH}_{2} \mathrm{~N}-\right)$, $4.87\left(2 \mathrm{H}, \mathrm{t}, J=5.2 \mathrm{~Hz},-\mathrm{OCH}_{2} \mathrm{CH}_{2} \mathrm{~N}-\right), 5.36\left(2 \mathrm{H}, \mathrm{s},-\mathrm{OCH}_{2} \mathrm{CN}-\right), 5.87(1 \mathrm{H}, \mathrm{s},-\mathrm{C}=\mathrm{CH}), 6.91-6.97(3 \mathrm{H}, \mathrm{m}$, $\mathrm{ArH}), 7.03(1 \mathrm{H}, \mathrm{d}, J=8.4 \mathrm{~Hz}, \mathrm{ArH}), 7.23(1 \mathrm{H}, \mathrm{t}, J=8.0 \mathrm{~Hz}, \mathrm{ArH}), 7.31(1 \mathrm{H}, \mathrm{d}, J=8.4 \mathrm{~Hz}, \mathrm{ArH}), 7.48-7.54$ $(2 \mathrm{H}, \mathrm{m}, \mathrm{ArH}), 7.54(1 \mathrm{H}, \mathrm{d}, J=15.2 \mathrm{~Hz},-\mathrm{CH}=\mathrm{CHCO}-), 7.61(2 \mathrm{H}, \mathrm{d}, J=8.4 \mathrm{~Hz}, \mathrm{ArH}), 7.78(1 \mathrm{H}, \mathrm{dd}, J=8.0$, $1.6 \mathrm{~Hz}, \mathrm{ArH}), 7.86(1 \mathrm{H}, \mathrm{d}, J=15.6 \mathrm{~Hz},-\mathrm{CH}=\mathrm{CHCO}-), 7.91(1 \mathrm{H}, \mathrm{dd}, J=8.0,1.2 \mathrm{~Hz}, \mathrm{ArH}), 7.94(1 \mathrm{H}, \mathrm{s}$, -CHN-), $12.87(1 \mathrm{H}, \mathrm{s}, \mathrm{OH}) .{ }^{13} \mathrm{C}-\mathrm{NMR}\left(500 \mathrm{MHz}, \mathrm{CDCl}_{3}\right): \delta 49.9,62.5,66.3,91.2,115.0,115.4,116.8,118.4$, 118.6, 118.8, 120.0, 123.1, 123.9, 124.7, 128.5, 129.5, 130.6, 132.6, 136.3, 141.6, 144.7, 153.3, 159.8, 162.6, 163.5, 164.9, 193.6. LCMS (ES+) $m / z=510.2\left([\mathrm{M}+\mathrm{H}]^{+}, t_{\mathrm{R}}=1.65 \mathrm{~min}\right.$ ). HRMS (ESI+) $\mathrm{m} / z=510.1651$ $[\mathrm{M}+\mathrm{H}]^{+}$found, $\mathrm{C}_{29} \mathrm{H}_{24} \mathrm{O}_{6} \mathrm{~N}_{3}{ }^{+}$required 510.1660.

4-((1-(2-(4-(3-Hydroxy-4-oxo-4H-chromen-2-yl)phenoxy)ethyl)-1H-1,2,3-triazol-4-yl)methoxy)-2H-chromen-2one (127). A mixture of alkyne coumarin $48(186 \mathrm{mg}, 0.931 \mathrm{mmol})$, azide flavonol $79(302 \mathrm{mg}$, $0.933 \mathrm{mmol}), \mathrm{CuSO}_{4} \cdot 5 \mathrm{H}_{2} \mathrm{O}(318 \mathrm{mg}, 1.27 \mathrm{mmol})$ and sodium ascorbate $(497 \mathrm{mg}, 2.51 \mathrm{mmol})$ in $t-\mathrm{BuOH} / \mathrm{H}_{2} \mathrm{O}(1: 1,40 \mathrm{~mL})$ was reacted according to GP-A. The crude residue was purified by flash column chromatography $\left(\mathrm{SiO}_{2}, 1 \%-5 \% \mathrm{MeOH} / \mathrm{CH}_{2} \mathrm{Cl}_{2}\right)$ and recrystallized from $\mathrm{MeOH}$ to afford triazole hybrid $127(77.9 \mathrm{mg}, 16 \%)$ as an off-white powdery solid. m.p. 228-230 ${ }^{\circ} \mathrm{C}$. TLC $R_{\mathrm{f}}=0.46$ $\left(5 \% \mathrm{MeOH} / \mathrm{CH}_{2} \mathrm{Cl}_{2}\right.$ ). IR $v_{\max }$ (neat) $/ \mathrm{cm}^{-1}$ : 3271w (O-H str), 3015w (C-H str), 2923w (C-H str), 1714s 
( $C=O$ str) $1622 \mathrm{~m}, 1603 \mathrm{~s}, 1565 \mathrm{~s}$ ( $\mathrm{C}=\mathrm{C}$ str), 1509m (C=C str), 1480m, 1470w, 1428m, 1406w, 1376w, 1262s, 1228m, 1177m, 1140w, 1106m, 1043m. ${ }^{1} \mathrm{H}-\mathrm{NMR}\left(500 \mathrm{MHz}, \mathrm{CDCl}_{3}\right): \delta 4.52(2 \mathrm{H}, \mathrm{t}, J=5.0 \mathrm{~Hz}$, $\left.-\mathrm{OCH}_{2} \mathrm{CH}_{2} \mathrm{~N}-\right), 4.89\left(2 \mathrm{H}, \mathrm{t}, \mathrm{J}=5.0 \mathrm{~Hz},-\mathrm{OCH}_{2} \mathrm{CH}_{2} \mathrm{~N}-\right), 5.37\left(2 \mathrm{H}, \mathrm{s},-\mathrm{OCH}_{2} \mathrm{CN}-\right), 5.89(1 \mathrm{H}, \mathrm{s},-\mathrm{C}=\mathrm{CH})$, $6.97(1 \mathrm{H}, \mathrm{br} \mathrm{s}, \mathrm{OH}), 7.03(2 \mathrm{H}, \mathrm{d}, J=9.0 \mathrm{~Hz}, \mathrm{ArH}), 7.24(1 \mathrm{H}, \mathrm{t}, J=8.0 \mathrm{~Hz}, \mathrm{ArH}), 7.31(1 \mathrm{H}, \mathrm{dd}, J=8.5$, $1.0 \mathrm{~Hz}, \mathrm{ArH}), 7.43(1 \mathrm{H}, \mathrm{t}, J=8.0 \mathrm{~Hz}, \mathrm{ArH}), 7.54(1 \mathrm{H}, \mathrm{t}, J=8.5 \mathrm{~Hz}, \mathrm{ArH}), 7.59(1 \mathrm{H}, \mathrm{d}, J=8.5 \mathrm{~Hz}, \mathrm{ArH})$, $7.72(1 \mathrm{H}, \mathrm{t}, J=8.5 \mathrm{~Hz}, \mathrm{ArH}), 7.78(1 \mathrm{H}, \mathrm{dd}, J=8.0,1.5 \mathrm{~Hz}, \mathrm{ArH}), 7.96(1 \mathrm{H}, \mathrm{s},-\mathrm{CHN}-), 8.23-8.27(3 \mathrm{H}, \mathrm{m}$, ArH). ${ }^{13} \mathrm{C}-\mathrm{NMR}\left(500 \mathrm{MHz}, \mathrm{CDCl}_{3}\right): \delta 49.9,62.6,66.3,91.2,114.5,115.4,116.8,118.2,120.7,123.1,123.9$, 124.5, 124.7, 124.8, 125.4, 129.7, 132.5, 133.5, 137.8, 141.7, 144.6, 153.3, 155.3, 159.0, 162.6, 164.9, 173.2. LCMS (ES+) $m / z=524.2\left([\mathrm{M}+\mathrm{H}]^{+}, t_{\mathrm{R}}=1.55 \mathrm{~min}\right)$. HRMS (ESI +$) m / z=524.1476[\mathrm{M}+\mathrm{H}]^{+}$found, $\mathrm{C}_{29} \mathrm{H}_{22} \mathrm{~N}_{3} \mathrm{O}_{7}{ }^{+}$required 524.1458.

4-(2-(4-(((4-Oxo-2-phenyl-4H-chromen-3-yl)oxy)methyl)-1H-1,2,3-triazol-1-yl)ethoxy)-2H-chromen-2-one (128). A mixture of alkyne flavone $30(310 \mathrm{mg}, 1.12 \mathrm{mmol})$, azide coumarin $58(256 \mathrm{mg}, 1.11 \mathrm{mmol})$, $\mathrm{CuSO}_{4} \cdot 5 \mathrm{H}_{2} \mathrm{O}(399 \mathrm{mg}, 1.60 \mathrm{mmol})$ and sodium ascorbate $(578 \mathrm{mg}, 2.92 \mathrm{mmol})$ in $t-\mathrm{BuOH} / \mathrm{H}_{2} \mathrm{O}$ (1:1, $40 \mathrm{~mL})$ was reacted according to GP-A. The crude residue was purified by flash column chromatography $\left(\mathrm{SiO}_{2}, 1 \%-5 \% \mathrm{MeOH} / \mathrm{CH}_{2} \mathrm{Cl}_{2}\right)$ to afford triazole hybrid 128 (354 mg, 63\%) as a white fluffy solid. m.p. $268-270{ }^{\circ} \mathrm{C}$. TLC $R_{\mathrm{f}}=0.30\left(5 \% \mathrm{MeOH} / \mathrm{CH}_{2} \mathrm{Cl}_{2}\right)$. IR $v_{\max }$ (neat) $/ \mathrm{cm}^{-1}: 3082 \mathrm{w}$ (C-H str), 2926w (C-H str), 1723s (C=O str), 1626s, 1567m (C=C str), 1494w, 1465m, 1400w, 1385m, $1274 \mathrm{~m}, 1239 \mathrm{~s}, 1197 \mathrm{~s}, 1182 \mathrm{~s}, 1148 \mathrm{~s}, 1140 \mathrm{~m}, 1111 \mathrm{~m}, 1058 \mathrm{w}, 1030 \mathrm{w} .{ }^{1} \mathrm{H}-\mathrm{NMR}\left(500 \mathrm{MHz}, \mathrm{CDCl}_{3}\right): \delta 4.47$ $\left(2 \mathrm{H}, \mathrm{t}, J=5.0 \mathrm{~Hz},-\mathrm{OCH}_{2} \mathrm{CH}_{2} \mathrm{~N}-\right), 4.85\left(2 \mathrm{H}, \mathrm{t}, J=5.0 \mathrm{~Hz},-\mathrm{OCH}_{2} \mathrm{CH}_{2} \mathrm{~N}-\right), 5.31\left(2 \mathrm{H}, \mathrm{s},-\mathrm{OCH}_{2} \mathrm{CN}-\right), 5.65$ $(1 \mathrm{H}, \mathrm{s},-\mathrm{C}=\mathrm{CH}), 7.24\left(1 \mathrm{H}, \mathrm{t}, J=7.5 \mathrm{~Hz}, \mathrm{ArH}\right.$, overlain by $\left.\mathrm{CDCl}_{3}\right), 7.33(1 \mathrm{H}, \mathrm{d}, J=8.5 \mathrm{~Hz}, \mathrm{ArH}), 7.41-7.46$ $(4 \mathrm{H}, \mathrm{m}, \mathrm{ArH}), 7.51(1 \mathrm{H}, \mathrm{d}, J=8.5 \mathrm{~Hz}, \mathrm{ArH}), 7.55(1 \mathrm{H}, \mathrm{t}, J=8.0 \mathrm{~Hz}, \mathrm{ArH}), 7.68-7.73(2 \mathrm{H}, \mathrm{m}, \mathrm{ArH}), 7.93$ $(1 \mathrm{H}, \mathrm{s},-\mathrm{CHN}-), 8.02-8.04(2 \mathrm{H}, \mathrm{m}, \mathrm{ArH}), 8.25(1 \mathrm{H}, \mathrm{d}, J=7.5 \mathrm{~Hz}, \mathrm{ArH}) .{ }^{13} \mathrm{C}-\mathrm{NMR}\left(500 \mathrm{MHz}, \mathrm{CDCl}_{3}\right): \delta$ 48.7, 65.2, 67.0, 91.1, 115.0, 116.8, 118.1, 122.9, 124.0, 124.2, 124.7, 124.9, 125.6, 128.4, 128.7, 130.7, 130.8, 132.8, 133.7, 139.7, 144.7, 153.3, 155.3, 156.3, 162.2, 164.6, 175.1. LCMS (ES+) $m / z=508.3\left([\mathrm{M}+\mathrm{H}]^{+}\right.$, $t_{\mathrm{R}}=1.81 \mathrm{~min}$ ). HRMS (ESI+) $m / z=508.1506[\mathrm{M}+\mathrm{H}]^{+}$found, $\mathrm{C}_{29} \mathrm{H}_{22} \mathrm{~N}_{3} \mathrm{O}_{6}{ }^{+}$required 508.1509.

(Z)-4-((1-(2-(4-((6-Methoxy-3-oxobenzofuran-2(3H)-ylidene)methyl)phenoxy)ethyl)-1H-1,2,3-triazol-4-yl)meth oxy)-2H-chromen-2-one (129). A mixture of alkyne coumarin 48 (181 $\mathrm{mg}, 0.902 \mathrm{mmol})$, azide aurone 89 ( $302 \mathrm{mg}, 0.895 \mathrm{mmol}), \mathrm{CuSO}_{4} \cdot 5 \mathrm{H}_{2} \mathrm{O}(250 \mathrm{mg}, 1.00 \mathrm{mmol})$ and sodium ascorbate ( $459 \mathrm{mg}, 2.32 \mathrm{mmol}$ ) in $t$ - $\mathrm{BuOH} / \mathrm{H}_{2} \mathrm{O}(1: 1,40 \mathrm{~mL})$ was reacted according to GP-A. The crude residue was purified by flash column chromatography $\left(\mathrm{SiO}_{2}, 1 \%-5 \% \mathrm{MeOH} / \mathrm{CH}_{2} \mathrm{Cl}_{2}\right)$ and recrystallized from $\mathrm{MeOH}$ to afford triazole hybrid $129(253 \mathrm{mg}, 53 \%)$ as a bright yellow-orange microcrystalline solid. m.p. $138-140{ }^{\circ} \mathrm{C}$. TLC $R_{\mathrm{f}}=0.40\left(5 \% \mathrm{MeOH} / \mathrm{CH}_{2} \mathrm{Cl}_{2}\right)$. IR $v_{\max }$ (neat) $/ \mathrm{cm}^{-1}: 3077 \mathrm{w}(\mathrm{C}-\mathrm{H} \mathrm{str}), 2926 \mathrm{w}(\mathrm{C}-\mathrm{H} \mathrm{str}), 1719 \mathrm{~s}$ (C=O str), 1650m, 1623m, 1593s ( $\mathrm{C}=\mathrm{C}$ str), $1565 \mathrm{~m}$ (C=C str), $1511 \mathrm{~m}$ (C=C str), $1442 \mathrm{~m}, 1399 \mathrm{~m}, 1269 \mathrm{~m}$, 1247s, 1182s, 1130s, 1096s, 1042m, 1019m. ${ }^{1} \mathrm{H}-\mathrm{NMR}\left(500 \mathrm{MHz}, \mathrm{CDCl}_{3}\right): \delta 3.94\left(3 \mathrm{H}, \mathrm{s},-\mathrm{OCH}_{3}\right), 4.48$ $\left(2 \mathrm{H}, \mathrm{t}, J=4.5 \mathrm{~Hz},-\mathrm{OCH}_{2} \mathrm{CH}_{2} \mathrm{~N}-\right), 4.87\left(2 \mathrm{H}, \mathrm{t}, J=4.5 \mathrm{~Hz},-\mathrm{OCH}_{2} \mathrm{CH}_{2} \mathrm{~N}-\right), 5.36\left(2 \mathrm{H}, \mathrm{s},-\mathrm{OCH}_{2} \mathrm{CN}-\right), 5.88$ $(1 \mathrm{H}, \mathrm{s},-\mathrm{C}=\mathrm{CH}), 6.75-6.78(2 \mathrm{H}, \mathrm{m}, \mathrm{ArH}), 6.76(1 \mathrm{H}, \mathrm{s},-\mathrm{C}=\mathrm{CH}), 6.94(2 \mathrm{H}, \mathrm{d}, J=9.0 \mathrm{~Hz}, \mathrm{ArH}), 7.23(1 \mathrm{H}, \mathrm{t}$, $J=8.0 \mathrm{~Hz}, \mathrm{ArH}), 7.31(1 \mathrm{H}, \mathrm{dd}, J=8.5,1.0 \mathrm{~Hz}, \mathrm{ArH}), 7.53(1 \mathrm{H}, \mathrm{t}, J=8.5 \mathrm{~Hz}, \mathrm{ArH}), 7.71(1 \mathrm{H}, \mathrm{d}, J=9.0 \mathrm{~Hz}$, $\mathrm{ArH}), 7.77(1 \mathrm{H}, \mathrm{dd}, J=8.0,1.5 \mathrm{~Hz}, \mathrm{ArH}), 7.84(2 \mathrm{H}, \mathrm{d}, J=8.5 \mathrm{~Hz}, \mathrm{ArH}), 7.95\left(1 \mathrm{H}, \mathrm{s},-\mathrm{CHN}-{ }^{13} \mathrm{C}-\mathrm{NMR}\right.$ $\left(500 \mathrm{MHz} \mathrm{CDCl}_{3}\right): \delta 49.9,56.0,62.5,66.3,91.2,96.6,111.3,112.1,114.8,115.0,115.4,116.8,123.1,123.9$, 124.7, 125.8, 126.3, 132.5, 133.1, 141.6, 147.1, 153.3, 158.6, 162.6, 164.9, 167.3, 168.3, 182.8. LCMS (ES+) $m / z=538.2\left([\mathrm{M}+\mathrm{H}]^{+}, t_{\mathrm{R}}=1.78 \mathrm{~min}\right)$. HRMS (ESI +$) m / z=538.1617[\mathrm{M}+\mathrm{H}]^{+}$found, $\mathrm{C}_{30} \mathrm{H}_{24} \mathrm{~N}_{3} \mathrm{O}_{7}{ }^{+}$ required 538.1614 .

4-(2-(4-(((3-(4-Methoxyphenyl)-4-oxo-4H-chromen-7-yl)oxy)methyl)-1H-1,2,3-triazol-1-yl)ethoxy)-2H-chromen -2-one (130). A mixture of alkyne isoflavone 46 (301 $\mathrm{mg}, 0.983 \mathrm{mmol})$, azide coumarin 58 (232 $\mathrm{mg}$, $1.00 \mathrm{mmol}), \mathrm{CuSO}_{4} \cdot 5 \mathrm{H}_{2} \mathrm{O}(288 \mathrm{mg}, 1.15 \mathrm{mmol})$ and sodium ascorbate $(503 \mathrm{mg}, 2.54 \mathrm{mmol})$ in $t-\mathrm{BuOH} / \mathrm{H}_{2} \mathrm{O}(1: 1,40 \mathrm{~mL})$ was reacted according to GP-A. The crude residue was purified by flash column chromatography $\left(\mathrm{SiO}_{2}, 1 \%-5 \% \mathrm{MeOH} / \mathrm{CH}_{2} \mathrm{Cl}_{2}\right)$ to afford triazole hybrid $130(363 \mathrm{mg}, 69 \%)$ as an off-white powdery solid. m.p. $208-210{ }^{\circ} \mathrm{C}$. TLC $R_{\mathrm{f}}=0.28\left(5 \% \mathrm{MeOH} / \mathrm{CH}_{2} \mathrm{Cl}_{2}\right)$. IR $v_{\max }$ (neat) $/ \mathrm{cm}^{-1}$ : 3085w (C-H str), 2927w (C-H str), 1730s (C=O str), 1621s, $1566 \mathrm{~m}(\mathrm{C}=\mathrm{C}$ str), $1513 \mathrm{~m}(\mathrm{C}=\mathrm{C}$ 
str), 1495w, 1444m, 1379m, 1330w, 1279w, 1241s, 1198m, 1181s, 1144m, 1109m, 1031m. ${ }^{1} \mathrm{H}-\mathrm{NMR}$ $\left(500 \mathrm{MHz}, \mathrm{CDCl}_{3}\right): \delta 3.85\left(3 \mathrm{H}, \mathrm{s},-\mathrm{OCH}_{3}\right), 4.58\left(2 \mathrm{H}, \mathrm{t}, \mathrm{J}=4.4 \mathrm{~Hz},-\mathrm{OCH}_{2} \mathrm{CH}_{2} \mathrm{~N}-\right), 4.95(2 \mathrm{H}, \mathrm{t}, J=4.4 \mathrm{~Hz}$, $\left.-\mathrm{OCH}_{2} \mathrm{CH}_{2} \mathrm{~N}-\right), 5.35\left(2 \mathrm{H}, \mathrm{s},-\mathrm{OCH}_{2} \mathrm{CN}-\right), 5.69(1 \mathrm{H}, \mathrm{s},-\mathrm{C}=\mathrm{CH}), 6.97-6.99(3 \mathrm{H}, \mathrm{m}, \mathrm{ArH}), 7.03(1 \mathrm{H}, \mathrm{dd}$, $J=8.8,2.0 \mathrm{~Hz}, \mathrm{ArH}), 7.23(1 \mathrm{H}, \mathrm{d}, J=7.6 \mathrm{~Hz}, \mathrm{ArH}), 7.31(1 \mathrm{H}, \mathrm{d}, J=8.0 \mathrm{~Hz}, \mathrm{ArH}), 7.50(2 \mathrm{H}, \mathrm{d}, J=8.4 \mathrm{~Hz}$, $\mathrm{ArH}), 7.56(1 \mathrm{H}, \mathrm{t}, J=8.0 \mathrm{~Hz}, \mathrm{ArH}), 7.65(1 \mathrm{H}, \mathrm{d}, J=8.0 \mathrm{~Hz}, \mathrm{ArH}), 7.84(1 \mathrm{H}, \mathrm{s},-\mathrm{CHN}-), 7.90(1 \mathrm{H}, \mathrm{s}$, $-\mathrm{C}=\mathrm{CH}), 8.20(1 \mathrm{H}, \mathrm{d}, J=8.8 \mathrm{~Hz}, \mathrm{ArH}) .{ }^{13} \mathrm{C}-\mathrm{NMR}\left(500 \mathrm{MHz}, \mathrm{CDCl}_{3}\right): \delta 49.0,55.3,62.4,67.0,91.3,101.2$, $114.0,114.8,114.9,117.0,118.9,120.2,122.4,123.5,124.0,124.1,125.0,128.0,130.1,132.9,143.7,152.1$, $153.3,157.7,159.6,162.2,164.4,175.7 . \mathrm{LCMS}(\mathrm{ES}+) \mathrm{m} / z=538.0\left([\mathrm{M}+\mathrm{H}]^{+}, t_{\mathrm{R}}=4.19 \mathrm{~min}\right)$. HRMS (ESI+) $m / z=538.1624[\mathrm{M}+\mathrm{H}]^{+}$found, $\mathrm{C}_{30} \mathrm{H}_{24} \mathrm{~N}_{3} \mathrm{O}_{7}{ }^{+}$required 538.1614.

(E)-4-((1-(2-(4-(3-Oxo-3-(2-(prop-2-yn-1-yloxy)phenyl)prop-1-en-1-yl)phenoxy)ethyl)-1H-1,2,3-triazol-4-yl) methoxy)-2H-chromen-2-one (131). A mixture of biflavonoid 126 (264 mg, $0.517 \mathrm{mmol})$, propargyl bromide $(0.130 \mathrm{~mL}, 1.46 \mathrm{mmol})$ and anhydrous $\mathrm{K}_{2} \mathrm{CO}_{3}(291 \mathrm{mg}, 2.10 \mathrm{mmol})$ in dry acetone $(50 \mathrm{~mL}) \mathrm{was}$ reacted according to GP-B. The crude residue was purified by flash column chromatography $\left(\mathrm{SiO}_{2}\right.$, $\left.1 \% \mathrm{MeOH} / \mathrm{CH}_{2} \mathrm{Cl}_{2}\right)$ to afford alkyne biflavonoid $131(229 \mathrm{mg}, 81 \%)$ as a pale yellow-white powdery solid. m.p. $164-166{ }^{\circ} \mathrm{C}$. TLC $R_{\mathrm{f}}=0.15\left(5 \% \mathrm{MeOH} / \mathrm{CH}_{2} \mathrm{Cl}_{2}\right)$. IR $v_{\max }$ (neat)/cm ${ }^{-1}: 3239 \mathrm{w}(\mathrm{C} \equiv \mathrm{C}-\mathrm{H}$ str), 3074w (C-H str), 2973w (C-H str), 2165w (C $\equiv \mathrm{C}$ str), 1723s (C=O str), 1651m, 1624m, 1600s (C=C str), 1566m (C=C str), 1510m (C=C str), 1482w, 1452m, 1401s, 1372m, 1328m, 1231s, 1175w, 1105m, 1028m. ${ }^{1} \mathrm{H}-\mathrm{NMR}\left(500 \mathrm{MHz}, \mathrm{CDCl}_{3}\right): \delta 2.54\left(1 \mathrm{H}, \mathrm{t}, J=2.0 \mathrm{~Hz},-\mathrm{OCH}_{2} \mathrm{C} \equiv \mathrm{CH}\right), 4.45(2 \mathrm{H}, \mathrm{t}, J=4.8 \mathrm{~Hz}$, $\left.-\mathrm{OCH}_{2} \mathrm{CH}_{2} \mathrm{~N}-\right), 4.79\left(2 \mathrm{H}, \mathrm{d}, \mathrm{J}=2.0 \mathrm{~Hz},-\mathrm{OCH}_{2} \mathrm{C} \equiv \mathrm{CH}\right), 4.85\left(2 \mathrm{H}, \mathrm{t}, J=4.8 \mathrm{~Hz},-\mathrm{OCH}_{2} \mathrm{CH}_{2} \mathrm{~N}-\right), 5.35(2 \mathrm{H}$, s, $\left.-\mathrm{OCH}_{2} \mathrm{CN}-\right), 5.87(1 \mathrm{H}, \mathrm{s},-\mathrm{C}=\mathrm{CH}), 6.88(2 \mathrm{H}, \mathrm{d}, J=8.8 \mathrm{~Hz}, \mathrm{ArH}), 7.09-7.13(2 \mathrm{H}, \mathrm{m}, \mathrm{ArH}), 7.23(1 \mathrm{H}, \mathrm{t}$, $J=7.6 \mathrm{~Hz}, \mathrm{ArH}), 7.29\left(1 \mathrm{H}, \mathrm{d}, J=13.2 \mathrm{~Hz},-\mathrm{CH}=\mathrm{CHCO}-\right.$, overlain by $\left.\mathrm{CDCl}_{3}\right), 7.32(1 \mathrm{H}, \mathrm{t}, J=4.4 \mathrm{~Hz}$, $\mathrm{ArH}), 7.49(1 \mathrm{H}, \mathrm{t}, J=8.8 \mathrm{~Hz}, \mathrm{ArH}), 7.53-7.58(3 \mathrm{H}, \mathrm{m}, \mathrm{ArH}), 7.56(1 \mathrm{H}, \mathrm{d}, J=15.6 \mathrm{~Hz},-\mathrm{CH}=\mathrm{CHCO}-)$, $7.64(1 \mathrm{H}, \mathrm{dd}, J=7.6,1.2 \mathrm{~Hz}, \mathrm{ArH}), 7.77(1 \mathrm{H}, \mathrm{dd}, J=8.4,0.8 \mathrm{~Hz}, \mathrm{ArH}), 7.93(1 \mathrm{H}, \mathrm{s},-\mathrm{CHN}-) .{ }^{13} \mathrm{C}-\mathrm{NMR}$ $\left(500 \mathrm{MHz}_{,} \mathrm{CDCl}_{3}\right): \delta 49.9,56.4,62.5,66.3,76.1,78.1,91.2,113.3,114.8,115.4,116.8,121.8,123.1,123.9$, 124.6, 125.6, 129.0, 130.0, 130.2, 130.5, 132.6, 132.6, 141.6, 142.7, 153.3, 155.8, 159.3, 162.5, 164.9, 192.5. LCMS (ES+) $m / z=548.0\left([\mathrm{M}+\mathrm{H}]^{+}, t_{\mathrm{R}}=4.38 \mathrm{~min}\right)$. HRMS (ESI+) $m / z=548.1803[\mathrm{M}+\mathrm{H}]^{+}$found, $\mathrm{C}_{32} \mathrm{H}_{26} \mathrm{O}_{6} \mathrm{~N}_{3}{ }^{+}$required 548.1816.

(Z)-2-(4-((1-(2-(4-((3-Oxobenzofuran-2(3H)-ylidene)methyl)phenoxy)ethyl)-1H-1,2,3-triazol-4-yl)methoxy)ph enyl)-3-(prop-2-yn-1-yloxy)-4H-chromen-4-one (132). A mixture of biflavonoid 122 (212 mg, $0.354 \mathrm{mmol}$ ), propargyl bromide $(0.063 \mathrm{~mL}, 0.707 \mathrm{mmol})$ and anhydrous $\mathrm{K}_{2} \mathrm{CO}_{3}(153 \mathrm{mg}, 1.11 \mathrm{mmol})$ in dry acetone $(20 \mathrm{~mL})$ was reacted according to GP-B. The crude residue was purified by flash column chromatography $\left(\mathrm{SiO}_{2}, 1 \% \mathrm{MeOH} / \mathrm{CH}_{2} \mathrm{Cl}_{2}\right)$ to afford alkyne biflavonoid $132(168 \mathrm{mg}, 74 \%)$ as a bright yellow powdery solid. m.p. $150-152{ }^{\circ} \mathrm{C}$. TLC $R_{\mathrm{f}}=0.50\left(5 \% \mathrm{MeOH} / \mathrm{CH}_{2} \mathrm{Cl}_{2}\right)$. IR $v_{\max }(\mathrm{neat}) / \mathrm{cm}^{-1}$ : 3233w (C $\equiv \mathrm{C}-\mathrm{H}$ str), 2939w (C-H str), 2115w (C $\equiv \mathrm{C}$ str), 1697s (C=O str), 1635m, 1606s, 1598s (C=C str), 1566m (C=C str), 1508s (C=C str), 1472m, 1395m, 1343w, 1301m, 1250s, 1198m, 1184s, 1148m, 1133w, 1047m, 1017w. ${ }^{1} \mathrm{H}-\mathrm{NMR}\left(500 \mathrm{MHz}, \mathrm{CDCl}_{3}\right): \delta 2.34\left(1 \mathrm{H}, \mathrm{t}, J=2.4 \mathrm{~Hz},-\mathrm{OCH}_{2} \mathrm{C} \equiv \mathrm{CH}\right), 4.46(2 \mathrm{H}, \mathrm{t}$, $\left.J=4.8 \mathrm{~Hz},-\mathrm{OCH}_{2} \mathrm{CH}_{2} \mathrm{~N}-\right), 4.84\left(2 \mathrm{H}, \mathrm{t}, J=4.8 \mathrm{~Hz},-\mathrm{OCH}_{2} \mathrm{CH}_{2} \mathrm{~N}-\right), 5.00\left(2 \mathrm{H}, \mathrm{d}, J=2.4 \mathrm{~Hz},-\mathrm{OCH}_{2} \mathrm{C} \equiv \mathrm{CH}\right)$, $5.33\left(2 \mathrm{H}, \mathrm{s},-\mathrm{OCH}_{2} \mathrm{CN}-\right), 6.83(1 \mathrm{H}, \mathrm{s},-\mathrm{C}=\mathrm{CH}), 6.93(2 \mathrm{H}, \mathrm{d}, J=8.8 \mathrm{~Hz}, \mathrm{ArH}), 7.12(2 \mathrm{H}, \mathrm{d}, J=9.2 \mathrm{~Hz}$, ArH), $7.22(1 \mathrm{H}, \mathrm{t}, J=7.6 \mathrm{~Hz}, \mathrm{ArH}), 7.31(1 \mathrm{H}, \mathrm{d}, J=8.4 \mathrm{~Hz}, \mathrm{ArH}), 7.38(1 \mathrm{H}, \mathrm{t}, J=7.6 \mathrm{~Hz}, \mathrm{ArH}), 7.51$ $(1 \mathrm{H}, \mathrm{d}, J=8.4 \mathrm{~Hz}, \mathrm{ArH}), 7.63-7.69(2 \mathrm{H}, \mathrm{m}, \mathrm{ArH}), 7.80(1 \mathrm{H}, \mathrm{d}, J=7.6 \mathrm{~Hz}, \mathrm{ArH}), 7.86(2 \mathrm{H}, \mathrm{d}, J=6.8 \mathrm{~Hz}$, $\mathrm{ArH}), 7.87(1 \mathrm{H}, \mathrm{s},-\mathrm{CHN}-), 8.16(2 \mathrm{H}, \mathrm{d}, J=8.8 \mathrm{~Hz}, \mathrm{ArH}), 8.22(1 \mathrm{H}, \mathrm{dd}, J=8.0,1.6 \mathrm{~Hz}, \mathrm{ArH}) .{ }^{13} \mathrm{C}-\mathrm{NMR}$ $\left(500 \mathrm{MHz}_{,} \mathrm{CDCl}_{3}\right): \delta 49.8,59.0,62.0,66.3,76.0,78.7,112.6,112.9,114.6,114.9,117.9,121.8,123.4,123.8$, $123.9,124.1,124.6,124.7,125.7,126.1,130.7,133.4,136.7,138.0,146.1,155.1,156.4,158.9,160.1,165.8$, 174.7, 184.5. LCMS (ES+) $m / z=638.1\left([\mathrm{M}+\mathrm{H}]^{+}, t_{\mathrm{r}}=4.82 \mathrm{~min}\right)$. HRMS (ESI+) $m / z=638.1902[\mathrm{M}+\mathrm{H}]^{+}$ found, $\mathrm{C}_{38} \mathrm{H}_{28} \mathrm{O}_{7} \mathrm{~N}_{3}{ }^{+}$required 638.1922 .

(E)-1-(4-Methoxy-2-(prop-2-yn-1-yloxy)phenyl)-3-(4-methoxy-3-((1-(2-(2-methoxy-4-((E)-3-(2,4,6-trimethoxy phenyl)acryloyl)phenoxy)ethyl)-1H-1,2,3-triazol-4-yl)methoxy)phenyl)prop-2-en-1-one (133). A mixture of biflavonoid 92 (111 mg, $0.148 \mathrm{mmol})$, propargyl bromide $(0.050 \mathrm{~mL}, 0.561 \mathrm{mmol})$ and anhydrous $\mathrm{K}_{2} \mathrm{CO}_{3}(113 \mathrm{mg}, 0.815 \mathrm{mmol})$ in dry acetone $(50 \mathrm{~mL})$ was reacted according to GP-B. The crude 
residue was purified by flash column chromatography $\left(\mathrm{SiO}_{2}, 1 \% \mathrm{MeOH} / \mathrm{CH}_{2} \mathrm{Cl}_{2}\right)$ to afford alkyne biflavonoid $133(107 \mathrm{mg}, 92 \%)$ as a bright yellow powdery solid. m.p. $170-172{ }^{\circ} \mathrm{C}$. TLC $R_{\mathrm{f}}=0.29(5 \%$ $\mathrm{MeOH} / \mathrm{CH}_{2} \mathrm{Cl}_{2}$ ). IR $v_{\max }$ (neat) $/ \mathrm{cm}^{-1}: 3286 \mathrm{w}$ (C $\equiv \mathrm{C}-\mathrm{H}$ str), 3002w (C-H str), 2936w (C-H str), 2841w (C-H str), 2160w (C $\equiv$ C str), 1650m (C=O str), 1599s (C=C str), 1511m (C=C str), 1458m, 1441w, 1432w, $1418 \mathrm{w}, 1399 \mathrm{w}, 1377 \mathrm{w}, 1337 \mathrm{~m}, 1321 \mathrm{~m}, 1301 \mathrm{~m}, 1259 \mathrm{~s}, 1205 \mathrm{w}, 1159 \mathrm{~m}, 1124 \mathrm{~m}, 1027 \mathrm{~m} .{ }^{1} \mathrm{H}-\mathrm{NMR}(500 \mathrm{MHz}$, $\left.\mathrm{CDCl}_{3}\right): \delta 2.64\left(1 \mathrm{H}, \mathrm{t}, J=2.4 \mathrm{~Hz},-\mathrm{OCH}_{2} \mathrm{C} \equiv \mathrm{CH}\right), 3.87\left(6 \mathrm{H}, \mathrm{s}, 2 \times-\mathrm{OCH}_{3}\right), 3.89\left(3 \mathrm{H}, \mathrm{s},-\mathrm{OCH}_{3}\right), 3.91$ $\left(9 \mathrm{H}, \mathrm{s}, 3 \times-\mathrm{OCH}_{3}\right), 4.45\left(2 \mathrm{H}, \mathrm{t}, J=4.8 \mathrm{~Hz},-\mathrm{OCH}_{2} \mathrm{CH}_{2} \mathrm{~N}-\right), 4.83\left(2 \mathrm{H}, \mathrm{t}, J=4.8 \mathrm{~Hz},-\mathrm{OCH}_{2} \mathrm{CH}_{2} \mathrm{~N}-\right), 4.84$ $\left(2 \mathrm{H}, \mathrm{d}, J=2.4 \mathrm{~Hz},-\mathrm{OCH}_{2} \mathrm{C} \equiv \mathrm{CH}\right), 5.33\left(2 \mathrm{H}, \mathrm{s},-\mathrm{OCH}_{2} \mathrm{CN}-\right), 6.15(2 \mathrm{H}, \mathrm{s}, \mathrm{ArH}), 6.61-6.63(2 \mathrm{H}, \mathrm{m}, \mathrm{ArH})$, $6.83(1 \mathrm{H}, \mathrm{d}, J=8.0 \mathrm{~Hz}, \mathrm{ArH}), 6.87(1 \mathrm{H}, \mathrm{d}, J=8.0 \mathrm{~Hz}, \mathrm{ArH}), 7.18(1 \mathrm{H}, \mathrm{dd}, J=8.4,2.0 \mathrm{~Hz}, \mathrm{ArH}), 7.39$ $(1 \mathrm{H}, \mathrm{d}, J=1.6 \mathrm{~Hz}, \mathrm{ArH}), 7.46(1 \mathrm{H}, \mathrm{d}, J=15.6 \mathrm{~Hz},-\mathrm{CH}=\mathrm{CHCO}-), 7.56-7.64(2 \mathrm{H}, \mathrm{m}, \mathrm{ArH}), 7.61(1 \mathrm{H}, \mathrm{d}$, $J=16.0 \mathrm{~Hz},-\mathrm{CH}=\mathrm{CHCO}-), 7.79(1 \mathrm{H}, \mathrm{d}, J=9.2 \mathrm{~Hz}, \mathrm{ArH}), 7.85(1 \mathrm{H}, \mathrm{d}, J=15.6 \mathrm{~Hz},-\mathrm{CH}=\mathrm{CHCO}-), 8.03$ $(1 \mathrm{H}, \mathrm{s},-\mathrm{CHN}-), 8.23(1 \mathrm{H}, \mathrm{d}, J=16.0 \mathrm{~Hz},-\mathrm{CH}=\mathrm{CHCO}-) .{ }^{13} \mathrm{C}-\mathrm{NMR}\left(500 \mathrm{MHz}, \mathrm{CDCl}_{3}\right): \delta 49.7,55.6,55.8$, 55.9, 55.9, 56.5, 63.1, 67.5, 76.3, 78.1, 90.5, 100.1, 106.4, 106.6, 111.5, 111.7, 112.4, 112.8, 121.6, 122.3, 122.8, 124.0, 124.6, 125.3, 128.5, 132.9, 133.7, 135.6, 142.1, 144.1, 147.7, 149.5, 150.6, 151.5, 158.2, 158.3, 161.6, 163.0, 163.8, 190.0, 190.3. LCMS (ES+) $m / z=790.3\left([\mathrm{M}+\mathrm{H}]^{+}, t_{\mathrm{R}}=4.71 \mathrm{~min}\right)$. HRMS (ESI+) $m / z=790.2949[\mathrm{M}+\mathrm{H}]^{+}$found, $\mathrm{C}_{44} \mathrm{H}_{44} \mathrm{O}_{11} \mathrm{~N}_{3}{ }^{+}$required 790.2970.

2-(4-(2-(4-(((4-Oxo-2-phenyl-4H-chromen-3-yl)oxy)methyl)-1H-1,2,3-triazol-1-yl)ethoxy)phenyl)-3-(prop-2-yn -1-yloxy)-4H-chromen-4-one (134). A mixture of biflavonoid 110 (161 mg, $0.268 \mathrm{mmol})$, propargyl bromide $(0.045 \mathrm{~mL}, 0.500 \mathrm{mmol})$ and anhydrous $\mathrm{K}_{2} \mathrm{CO}_{3}(111 \mathrm{mg}, 0.803 \mathrm{mmol})$ in dry acetone $(20 \mathrm{~mL})$ was reacted according to GP-B. The crude residue was purified by flash column chromatography $\left(\mathrm{SiO}_{2}, 1 \% \mathrm{MeOH} / \mathrm{CH}_{2} \mathrm{Cl}_{2}\right)$ to afford alkyne biflavonoid $134(161 \mathrm{mg}, 94 \%)$ as a pale yellow-white powdery solid. m.p. $118-120^{\circ} \mathrm{C}$. TLC $R_{\mathrm{f}}=0.34\left(5 \% \mathrm{MeOH} / \mathrm{CH}_{2} \mathrm{Cl}_{2}\right)$. IR $v_{\max }$ (neat) $/ \mathrm{cm}^{-1}: 3233 \mathrm{w}$ (C $\equiv \mathrm{C}-\mathrm{H}$ str), 2939w (C-H str), 2116w ( $\mathrm{C} \equiv \mathrm{C}$ str), 1631s (C=O str), 1613s, 1600s, 1559m (C=C str), 1508m (C=C str), 1467s, 1392s, 1287w, 1252m, 1239m, 1184s, 1146m, 1123w, 1109w, 1042w. ${ }^{1} \mathrm{H}-\mathrm{NMR}(500 \mathrm{MHz}$, $\left.\mathrm{CDCl}_{3}\right): \delta 2.33\left(1 \mathrm{H}, \mathrm{t}, J=2.4 \mathrm{~Hz},-\mathrm{OCH}_{2} \mathrm{C} \equiv \mathrm{CH}\right), 4.39\left(2 \mathrm{H}, \mathrm{t}, J=5.2 \mathrm{~Hz},-\mathrm{OCH}_{2} \mathrm{CH}_{2} \mathrm{~N}-\right), 4.73(2 \mathrm{H}, \mathrm{t}$, $\left.J=5.2 \mathrm{~Hz},-\mathrm{OCH}_{2} \mathrm{CH}_{2} \mathrm{~N}-\right), 4.99\left(2 \mathrm{H}, \mathrm{d}, J=2.4 \mathrm{~Hz},-\mathrm{OCH}_{2} \mathrm{C} \equiv \mathrm{CH}\right), 5.33\left(2 \mathrm{H}, \mathrm{s},-\mathrm{OCH}_{2} \mathrm{CN}-\right) 6.97(2 \mathrm{H}, \mathrm{d}$, $J=9.2 \mathrm{~Hz}, \mathrm{ArH}), 7.39-7.45(5 \mathrm{H}, \mathrm{m}, \mathrm{ArH}), 7.53(1 \mathrm{H}, \mathrm{d}, J=8.0 \mathrm{~Hz}, \mathrm{ArH}), 7.54(1 \mathrm{H}, \mathrm{d}, J=8.0 \mathrm{~Hz}, \mathrm{ArH})$, 7.67-7.72 (2H, m, ArH), $7.85(1 \mathrm{H}, \mathrm{s},-\mathrm{CHN}-), 8.02-8.04(2 \mathrm{H}, \mathrm{m}, \mathrm{ArH}), 8.15(2 \mathrm{H}, \mathrm{d}, J=9.2 \mathrm{~Hz}, \mathrm{ArH}), 8.25$ $(1 \mathrm{H}, \mathrm{dd}, J=8.0,1.2 \mathrm{~Hz}, \mathrm{ArH}), 8.28(1 \mathrm{H}, \mathrm{dd}, J=8.0,1.2 \mathrm{~Hz}, \mathrm{ArH}) .{ }^{13} \mathrm{C}-\mathrm{NMR}\left(500 \mathrm{MHz}, \mathrm{CDCl}_{3}\right): \delta 49.5$, 59.0, 65.1, 66.2, 76.1, 78.6, 114.3, 117.9, 118.1, 124.0, 124.1, 124.3, 124.7, 124.8, 124.9, 125.7, 128.3, 128.7, 130.7, 130.8, 133.4, 133.6, 138.1, 139.5, 144.1, 155.1, 155.3, 156.2, 156.4, 159.6, 174.7, 175.1. LCMS (ES+) $m / z=638.1\left([\mathrm{M}+\mathrm{H}]^{+}, t_{\mathrm{R}}=4.70 \mathrm{~min}\right)$. HRMS (ESI +$) m / z=638.1905[\mathrm{M}+\mathrm{H}]^{+}$found, $\mathrm{C}_{38} \mathrm{H}_{28} \mathrm{O}_{7} \mathrm{~N}_{3}{ }^{+}$ required 638.1922.

(E)-2-(4-(2-(4-((4-(3-(Ferrocenyl)acryloyl)-2-methoxyphenoxy)methyl)-1H-1,2,3-triazol-1-yl)ethoxy)phenyl)-3(prop-2-yn-1-yloxy)-4H-chromen-4-one (135). A mixture of biflavonoid 106 (190 mg, $0.263 \mathrm{mmol}$ ), propargyl bromide $(0.047 \mathrm{~mL}, 0.526 \mathrm{mmol})$ and anhydrous $\mathrm{K}_{2} \mathrm{CO}_{3}(127 \mathrm{mg}, 0.920 \mathrm{mmol})$ in dry acetone $(20 \mathrm{~mL})$ was reacted according to GP-B. The crude residue was purified by flash column chromatography $\left(\mathrm{SiO}_{2}, 1 \% \mathrm{MeOH} / \mathrm{CH}_{2} \mathrm{Cl}_{2}\right)$ to afford alkyne biflavonoid $135(95.5 \mathrm{mg}, 48 \%)$ as a dark red powdery solid. m.p. $108-110{ }^{\circ} \mathrm{C}$. TLC $R_{\mathrm{f}}=0.37\left(5 \% \mathrm{MeOH} / \mathrm{CH}_{2} \mathrm{Cl}_{2}\right)$. IR $v_{\max }$ (neat) $/ \mathrm{cm}^{-1}: 3292 \mathrm{w}$ ( $\mathrm{C} \equiv \mathrm{C}-\mathrm{H}$ str), 2937w (C-H str), 2245w ( $\equiv \mathrm{C}$ str), 1636m ( $\mathrm{C}=\mathrm{O}$ str), 1598s ( $\mathrm{C}=\mathrm{C}$ str), 1576s (C=C str), 1509s (C=C str), 1467m, 1419m, 1395m, 1349w, 1258s, 1198m, 1184m, 1150m, 1029w, 1000w. ${ }^{1} \mathrm{H}-\mathrm{NMR}$ $\left(500 \mathrm{MHz}, \mathrm{CDCl}_{3}\right): \delta 2.33\left(1 \mathrm{H}, \mathrm{t}, J=2.5 \mathrm{~Hz},-\mathrm{OCH}_{2} \mathrm{C} \equiv \mathrm{CH}\right), 3.96\left(3 \mathrm{H}, \mathrm{s},-\mathrm{OCH}_{3}\right), 4.17\left(5 \mathrm{H}, \mathrm{s},-\mathrm{C}_{5} \mathrm{H}_{5}\right)$, 4.45-4.47 $\left(4 \mathrm{H}, \mathrm{m},-\mathrm{C}_{5} \mathrm{H}_{4}\right.$ and $\left.-\mathrm{OCH}_{2} \mathrm{CH}_{2} \mathrm{~N}-\right), 4.57\left(2 \mathrm{H}, \mathrm{t}, J=2.0 \mathrm{~Hz},-\mathrm{C}_{5} \mathrm{H}_{4}\right), 4.82(2 \mathrm{H}, \mathrm{t}, J=5.0 \mathrm{~Hz}$, $\left.-\mathrm{OCH}_{2} \mathrm{CH}_{2} \mathrm{~N}-\right), 4.98\left(2 \mathrm{H}, \mathrm{d}, J=2.5 \mathrm{~Hz},-\mathrm{OCH}_{2} \mathrm{C} \equiv \mathrm{CH}\right), 5.42\left(2 \mathrm{H}, \mathrm{s},-\mathrm{OCH}_{2} \mathrm{CN}-\right), 6.96(2 \mathrm{H}, \mathrm{d}, J=9.0 \mathrm{~Hz}$, $\mathrm{ArH}), 7.13(1 \mathrm{H}, \mathrm{d}, J=15.5 \mathrm{~Hz},-\mathrm{CH}=\mathrm{CHCO}-), 7.15(1 \mathrm{H}, \mathrm{d}, J=8.5 \mathrm{~Hz}, \mathrm{ArH}), 7.41(1 \mathrm{H}, \mathrm{t}, J=8.5 \mathrm{~Hz}, \mathrm{ArH})$, $7.54(1 \mathrm{H}, \mathrm{dd}, J=8.5,0.5 \mathrm{~Hz}, \mathrm{ArH}), 7.58(1 \mathrm{H}, \mathrm{dd}, J=8.5,2.0 \mathrm{~Hz}, \mathrm{ArH}), 7.63(1 \mathrm{H}, \mathrm{d}, J=2.0 \mathrm{~Hz}, \mathrm{ArH})$, $7.69(1 \mathrm{H}, \mathrm{t}, J=8.5 \mathrm{~Hz}, \mathrm{ArH}), 7.73(1 \mathrm{H}, \mathrm{d}, J=15.0 \mathrm{~Hz},-\mathrm{CH}=\mathrm{CHCO}-), 7.90(1 \mathrm{H}, \mathrm{s},-\mathrm{CHN}-), 8.14(2 \mathrm{H}, \mathrm{d}$, $J=9.0 \mathrm{~Hz}, \mathrm{ArH}), 8.25(1 \mathrm{H}, \mathrm{dd}, J=8.0,1.5 \mathrm{~Hz}, \mathrm{ArH}) .{ }^{13} \mathrm{C}-\mathrm{NMR}\left(500 \mathrm{MHz}, \mathrm{CDCl}_{3}\right): \delta 49.7,56.1,59.0$, $62.8,66.2,68.9,69.7,71.2,76.1,78.6,79.3,111.2,112.3,114.2,117.9,118.5,122.4,124.0,124.3,124.4,124.7$, 
125.7, 130.8, 132.3, 133.4, 138.1, 143.8, 146.0, 149.5, 151.3, 155.1, 156.1, 159.5, 174.7, 187.9. LCMS (ES+) $m / z=762.1\left([\mathrm{M}+\mathrm{H}]^{+}, t_{\mathrm{R}}=4.88 \mathrm{~min}\right)$. HRMS (ESI+) $m / z=762.1878[\mathrm{M}+\mathrm{H}]^{+}$found, $\mathrm{C}_{43} \mathrm{H}_{36} \mathrm{O}_{7} \mathrm{~N}_{3} \mathrm{Fe}^{+}$ required 762.1897 .

(E)-4-((1-(2-(4-(3-Oxo-3-(2-((1-(2-((4-oxo-2-phenyl-4H-chromen-7-yl)oxy)ethyl)-1H-1,2,3-triazol-4-yl)methoxy) phenyl)prop-1-en-1-yl)phenoxy)ethyl)-1H-1,2,3-triazol-4-yl)methoxy)-2H-chromen-2-one (136). A mixture of alkyne biflavonoid 131 (205 mg, $0.375 \mathrm{mmol})$, azide flavone $86(123 \mathrm{mg}, 0.399 \mathrm{mmol}), \mathrm{CuSO}_{4} \cdot 5 \mathrm{H}_{2} \mathrm{O}$ $(115 \mathrm{mg}, 0.459 \mathrm{mmol})$ and sodium ascorbate $(192 \mathrm{mg}, 0.969 \mathrm{mmol})$ in $t-\mathrm{BuOH} / \mathrm{H}_{2} \mathrm{O}(1: 1,40 \mathrm{~mL})$ was reacted according to GP-A. The crude residue was purified by flash column chromatography $\left(\mathrm{SiO}_{2}\right.$, $\left.1 \%-5 \% \mathrm{MeOH} / \mathrm{CH}_{2} \mathrm{Cl}_{2}\right)$ to afford triazole hybrid $136(214 \mathrm{mg}, 67 \%)$ as a pale yellow-white powdery solid. m.p. $78-80{ }^{\circ} \mathrm{C}$. TLC $R_{\mathrm{f}}=0.37\left(7 \% \mathrm{MeOH} / \mathrm{CH}_{2} \mathrm{Cl}_{2}\right)$. IR $v_{\max }$ (neat) $/ \mathrm{cm}^{-1}: 3080 \mathrm{w}(\mathrm{C}-\mathrm{H} \mathrm{str})$, 2923w (C-H str), 1708s (C=O str), 1622s, 1597s (C=C str), 1567s (C=C str), 1509w (C=C str), 1493w, 1449m, 1356m, 1274w, 1237m, 1173m, 1139w, 1105w, 1043w, 1026w. ${ }^{1} \mathrm{H}-\mathrm{NMR}\left(500 \mathrm{MHz}, \mathrm{CDCl}_{3}\right): \delta$ $4.38\left(2 \mathrm{H}, \mathrm{t}, \mathrm{J}=5.2 \mathrm{~Hz},-\mathrm{OCH}_{2} \mathrm{CH}_{2} \mathrm{~N}-\right), 4.41\left(2 \mathrm{H}, \mathrm{t}, J=5.2 \mathrm{~Hz},-\mathrm{OCH}_{2} \mathrm{CH}_{2} \mathrm{~N}-\right), 4.70(2 \mathrm{H}, \mathrm{t}, J=5.2 \mathrm{~Hz}$, $\left.-\mathrm{OCH}_{2} \mathrm{CH}_{2} \mathrm{~N}-\right), 4.82\left(2 \mathrm{H}, \mathrm{t}, J=5.2 \mathrm{~Hz},-\mathrm{OCH}_{2} \mathrm{CH}_{2} \mathrm{~N}-\right), 5.34\left(2 \mathrm{H}, \mathrm{s},-\mathrm{OCH}_{2} \mathrm{CN}-\right), 5.34\left(2 \mathrm{H}, \mathrm{s},-\mathrm{OCH} \mathrm{CN}^{-}\right)$, $5.85(1 \mathrm{H}, \mathrm{s},-\mathrm{C}=\mathrm{CH}), 6.75(1 \mathrm{H}, \mathrm{s},-\mathrm{C}=\mathrm{CH}), 6.77-6.80(3 \mathrm{H}, \mathrm{m}, \mathrm{ArH}), 6.85(1 \mathrm{H}, \mathrm{d}, J=2.4 \mathrm{~Hz}, \mathrm{ArH}), 7.08(1 \mathrm{H}$, t, $J=7.6 \mathrm{~Hz}, \mathrm{ArH}), 7.14(1 \mathrm{H}, \mathrm{d}, J=8.4 \mathrm{~Hz}, \mathrm{ArH}), 7.20(1 \mathrm{H}, \mathrm{t}, J=8.0 \mathrm{~Hz}, \mathrm{ArH}), 7.26(1 \mathrm{H}, \mathrm{d}, J=16.0 \mathrm{~Hz}$, - $\mathrm{CH}=\mathrm{CHCO}-$, overlain by $\left.\mathrm{CDCl}_{3}\right), 7.28(1 \mathrm{H}, \mathrm{d}, J=4.0 \mathrm{~Hz}, \mathrm{ArH}), 7.38(2 \mathrm{H}, \mathrm{d}, J=8.4 \mathrm{~Hz}, \mathrm{ArH}), 7.46-7.54$ $(5 \mathrm{H}, \mathrm{m}, \mathrm{ArH}), 7.50(1 \mathrm{H}, \mathrm{d}, J=16.0 \mathrm{~Hz},-\mathrm{CH}=\mathrm{CHCO}-), 7.62(1 \mathrm{H}, \mathrm{dd}, J=8.8,1.6 \mathrm{~Hz}, \mathrm{ArH}), 7.74(1 \mathrm{H}, \mathrm{dd}$, $J=8.0,1.2 \mathrm{~Hz}, \mathrm{ArH}), 7.77(1 \mathrm{H}, \mathrm{s},-\mathrm{CHN}-), 7.89(2 \mathrm{H}, \mathrm{dd}, J=7.6,1.6 \mathrm{~Hz}, \mathrm{ArH}), 7.96(1 \mathrm{H}, \mathrm{s},-\mathrm{CHN}-), 8.03$ $(1 \mathrm{H}, \mathrm{d}, J=8.8 \mathrm{~Hz}, \mathrm{ArH}) .{ }^{13} \mathrm{C}-\mathrm{NMR}\left(500 \mathrm{MHz}, \mathrm{CDCl}_{3}\right): \delta 49.4,49.8,62.5,62.8,66.2,66.6,91.2,101.4$, $107.5,113.1,114.1,114.8,115.4,116.7,118.5,121.5,123.1,123.9,124.0,124.8,125.7,126.2,127.3,128.7$, $129.1,129.8,130.1,130.4,131.6,131.6,132.5,132.9,141.6,142.4,144.1,153.3,156.5,157.6,159.3,161.9$, $162.5,163.2,164.9,177.6,192.6$. LCMS (ES+) $m / z=855.3\left([\mathrm{M}+\mathrm{H}]^{+}, t_{\mathrm{R}}=4.57 \mathrm{~min}\right)$. HRMS (ESI+) $m / z=855.2743[\mathrm{M}+\mathrm{H}]^{+}$found, $\mathrm{C}_{49} \mathrm{H}_{39} \mathrm{O}_{9} \mathrm{~N}_{6}{ }^{+}$required 855.2773.

(Z)-7-(2-(4-((4-Oxo-2-(4-((1-(2-(4-((3-oxobenzofuran-2(3H)-ylidene)methyl)phenoxy)ethyl)-1H-1,2,3-triazol-4yl)methoxy)phenyl)-4H-chromen-3-yl)oxy)methyl)-1H-1,2,3-triazol-1-yl)ethoxy)-2-phenyl-4H-chromen-4-one (137). A mixture of alkyne biflavonoid 132 (145 mg, $0.227 \mathrm{mmol})$, azide flavone 86 (73.5 $\mathrm{mg}$, $0.239 \mathrm{mmol}), \mathrm{CuSO}_{4} \cdot 5 \mathrm{H}_{2} \mathrm{O}(70.1 \mathrm{mg}, 0.281 \mathrm{mmol})$ and sodium ascorbate $(129 \mathrm{mg}, 0.651 \mathrm{mmol})$ in $t-\mathrm{BuOH} / \mathrm{H}_{2} \mathrm{O}(1: 1,40 \mathrm{~mL})$ was reacted according to GP-A. The crude residue was purified by flash column chromatography $\left(\mathrm{SiO}_{2}, 1 \%-5 \% \mathrm{MeOH} / \mathrm{CH}_{2} \mathrm{Cl}_{2}\right)$ to afford triazole hybrid $137(106 \mathrm{mg}, 49 \%)$ as a bright yellow powdery solid. m.p. $198-200{ }^{\circ} \mathrm{C}$. TLC $R_{\mathrm{f}}=0.33\left(7 \% \mathrm{MeOH} / \mathrm{CH}_{2} \mathrm{Cl}_{2}\right)$. IR $v_{\max }$ (neat) $/ \mathrm{cm}^{-1}$ : 2931w (C-H str), 2874w (C-H str), 1698w, 1628s (C=O str), 1602s, 1594s (C=C str), 1508s (C=C str), 1466m, 1449m, 1423w, 1396w, 1374m, 1299w, 1288w, 1249s, 1178s, 1148w, 1128w, 1110w, 1096w, 1041m. ${ }^{1} \mathrm{H}-\mathrm{NMR}\left(500 \mathrm{MHz}, \mathrm{CDCl}_{3}\right): \delta 4.44-4.46\left(4 \mathrm{H}, \mathrm{m}, 2 \times-\mathrm{OCH}_{2} \mathrm{CH}_{2} \mathrm{~N}-\right), 4.78(2 \mathrm{H}, \mathrm{t}$, $\left.J=4.8 \mathrm{~Hz},-\mathrm{OCH}_{2} \mathrm{CH}_{2} \mathrm{~N}-\right), 4.83\left(2 \mathrm{H}, \mathrm{t}, J=4.8 \mathrm{~Hz},-\mathrm{OCH}_{2} \mathrm{CH}_{2} \mathrm{~N}-\right), 5.28\left(2 \mathrm{H}, \mathrm{s},-\mathrm{OCH}_{2} \mathrm{CN}-\right), 5.33(2 \mathrm{H}$, $\left.\mathrm{s},-\mathrm{OCH}_{2} \mathrm{CN}-\right), 6.75(1 \mathrm{H}, \mathrm{s},-\mathrm{C}=\mathrm{CH}), 6.81(1 \mathrm{H}, \mathrm{s},-\mathrm{C}=\mathrm{CH}), 6.91-6.94(4 \mathrm{H}, \mathrm{m}, \mathrm{ArH}), 7.04(2 \mathrm{H}, \mathrm{d}, J=9.2$ $\mathrm{Hz}, \mathrm{ArH}), 7.21(1 \mathrm{H}, \mathrm{d}, J=7.6 \mathrm{~Hz}, \mathrm{ArH}), 7.30(1 \mathrm{H}, \mathrm{d}, J=8.4 \mathrm{~Hz}, \mathrm{ArH}), 7.37(1 \mathrm{H}, \mathrm{d}, J=7.6 \mathrm{~Hz}, \mathrm{ArH})$, 7.47-7.53 (4H, m, ArH), 7.62-7.67 (2H, m, ArH), $7.79(1 \mathrm{H}, \mathrm{d}, J=7.6 \mathrm{~Hz}, \mathrm{ArH}), 7.84(2 \mathrm{H}, \mathrm{d}, J=8.8 \mathrm{~Hz}$, $\mathrm{ArH}), 7.87-7.90(3 \mathrm{H}, \mathrm{m},-\mathrm{CHN}-\mathrm{and} \mathrm{ArH}), 7.94(1 \mathrm{H}, \mathrm{s},-\mathrm{CHN}-), 8.04(2 \mathrm{H}, \mathrm{d}, J=8.8 \mathrm{~Hz}, \mathrm{ArH}), 8.11(1 \mathrm{H}$, $\mathrm{d}, J=8.8 \mathrm{~Hz}, \mathrm{ArH}), 8.23(1 \mathrm{H}, \mathrm{dd}, J=8.0,1.2 \mathrm{~Hz}, \mathrm{ArH}) .{ }^{13} \mathrm{C}-\mathrm{NMR}\left(500 \mathrm{MHz}, \mathrm{CDCl}_{3}\right): \delta 49.3,49.7$, $61.9,65.0,66.3,66.7,101.5,107.6,112.7,112.9,114.2,114.7,114.9,117.9,118.5,121.8,123.4,123.6,124.0$, $124.1,124.6,124.7,125.0,125.6,126.1,126.2,127.4,129.0,130.5,131.5,131.7,133.4,133.4,136.7,139.0$, 143.7, 144.3, 146.1, 155.1, 156.1, 157.7, 159.0, 160.0, 162.1, 163.2, 165.9, 174.9, 177.7, 184.5. LCMS (ES+) $m / z=945.4\left([\mathrm{M}+\mathrm{H}]^{+}, t_{\mathrm{R}}=4.89 \mathrm{~min}\right)$. HRMS (ESI+) $m / z=945.2851[\mathrm{M}+\mathrm{H}]^{+}$found, $\mathrm{C}_{55} \mathrm{H}_{41} \mathrm{O}_{10} \mathrm{~N}_{6}{ }^{+}$ required 945.2879 .

7-Methoxy-2-(4-(2-(4-((5-methoxy-2-((E)-3-(4-methoxy-3-((1-(2-(2-methoxy-4-((E)-3-(2,4,6-trimethoxyphenyl) acryloyl)phenoxy)ethyl)-1H-1,2,3-triazol-4-yl)methoxy)phenyl)acryloyl)phenoxy)methyl)-1H-1,2,3-triazol-1-yl) ethoxy)phenyl)-4H-chromen-4-one (138). A mixture of alkyne biflavonoid 133 (107 mg, $0.135 \mathrm{mmol}$ ), azide flavone $87(49.3 \mathrm{mg}, 0.146 \mathrm{mmol}), \mathrm{CuSO}_{4} \cdot 5 \mathrm{H}_{2} \mathrm{O}(66.0 \mathrm{mg}, 0.264 \mathrm{mmol})$ and sodium ascorbate 
(72.8 $\mathrm{mg}, 0.367 \mathrm{mmol})$ in $t-\mathrm{BuOH} / \mathrm{H}_{2} \mathrm{O}(1: 1,40 \mathrm{~mL})$ was reacted according to GP-A. The crude residue was purified by flash column chromatography $\left(\mathrm{SiO}_{2}, 1 \%-5 \% \mathrm{MeOH} / \mathrm{CH}_{2} \mathrm{Cl}_{2}\right)$ to afford triazole hybrid $138(8.00 \mathrm{mg}, 5 \%)$ as a pale yellow-white powdery solid. m.p. $128-130{ }^{\circ} \mathrm{C}$. TLC $R_{\mathrm{f}}=0.19(5 \%$ $\mathrm{MeOH} / \mathrm{CH}_{2} \mathrm{Cl}_{2}$ ). IR $v_{\max }$ (neat) $/ \mathrm{cm}^{-1}: 2927 \mathrm{w}$ (C-H str), 2846w (C-H str), 1736w, 1627m (C=O str), $1600 \mathrm{~s}$ (C=C str), 1510s (C=C str), 1454m, 1439m, 1423m, 1375w, 1355w, 1338w, 1258s, 1204m, 1162m, 1123s, 1023s. ${ }^{1} \mathrm{H}-\mathrm{NMR}\left(500 \mathrm{MHz}\right.$, DMSO-d $\left.d_{6}\right): \delta 3.76\left(6 \mathrm{H}, \mathrm{s}, 2 \times-\mathrm{OCH}_{3}\right), 3.85\left(6 \mathrm{H}, \mathrm{s}, 2 \times-\mathrm{OCH}_{3}\right), 3.90$ $\left(9 \mathrm{H}, \mathrm{s}, 3 \times-\mathrm{OCH}_{3}\right), 4.38\left(2 \mathrm{H}, \mathrm{t}, J=4.4 \mathrm{~Hz},-\mathrm{OCH}_{2} \mathrm{CH}_{2} \mathrm{~N}-\right), 4.50\left(2 \mathrm{H}, \mathrm{t}, J=4.8 \mathrm{~Hz},-\mathrm{OCH}_{2} \mathrm{CH}_{2} \mathrm{~N}-\right), 4.71$ $\left(2 \mathrm{H}, \mathrm{t}, J=4.8 \mathrm{~Hz},-\mathrm{OCH}_{2} \mathrm{CH}_{2} \mathrm{~N}-\right), 4.82\left(2 \mathrm{H}, \mathrm{t}, J=4.8 \mathrm{~Hz},-\mathrm{OCH}_{2} \mathrm{CH}_{2} \mathrm{~N}-\right), 5.24\left(2 \mathrm{H}, \mathrm{s},-\mathrm{OCH}_{2} \mathrm{CN}-\right), 5.38$ $\left(2 \mathrm{H}, \mathrm{s},-\mathrm{OCH}_{2} \mathrm{CN}-\right), 6.29(2 \mathrm{H}, \mathrm{s}, \mathrm{ArH}), 6.57-6.66(1 \mathrm{H}, \mathrm{m}, \mathrm{ArH}), 6.81(1 \mathrm{H}, \mathrm{s},-\mathrm{C}=\mathrm{CH}), 6.82-6.85(1 \mathrm{H}, \mathrm{m}$, ArH), 6.92-6.98 (3H, m, ArH), 7.02-7.11 (3H, m, ArH), $7.20(1 \mathrm{H}, \mathrm{d}, J=8.8 \mathrm{~Hz}, \mathrm{ArH}), 7.27(1 \mathrm{H}, \mathrm{dd}$, $J=5.6,2.4 \mathrm{~Hz}, \mathrm{ArH}), 7.47(1 \mathrm{H}, \mathrm{d}, J=15.6 \mathrm{~Hz},-\mathrm{CH}=\mathrm{CHCO}-), 7.48(1 \mathrm{H}, \mathrm{d}, J=8.0 \mathrm{~Hz}, \mathrm{ArH}), 7.54-7.64$ $(2 \mathrm{H}, \mathrm{m}, \mathrm{ArH}), 7.56(1 \mathrm{H}, \mathrm{d}, J=15.2 \mathrm{~Hz},-\mathrm{CH}=\mathrm{CHCO}-), 7.82(1 \mathrm{H}, \mathrm{d}, J=16.0 \mathrm{~Hz},-\mathrm{CH}=\mathrm{CHCO}-), 7.87-8.06$ $(3 \mathrm{H}, \mathrm{m}, \mathrm{ArH}$ and $-\mathrm{CHN}-), 8.02(1 \mathrm{H}, \mathrm{d}, J=16.0 \mathrm{~Hz},-\mathrm{CH}=\mathrm{CHCO}-), 8.28(1 \mathrm{H}, \mathrm{d}, J=6.4 \mathrm{~Hz}, \mathrm{ArH}), 8.31(1 \mathrm{H}$, s, -CHN-). ${ }^{13} \mathrm{C}-\mathrm{NMR}\left(500 \mathrm{MHz}\right.$, DMSO- $\left.d_{6}\right): \delta 48.9,49.0,55.5,55.7,56.0,61.5,61.7,66.2,67.1,91.0,99.8$, $100.9,105.1,105.4,106.6,111.0,111.3,111.8,112.0,112.7,114.5,114.9,114.9,115.0,115.0,117.1,120.2$, 122.1, 123.8, 125.3, 126.1, 127.6, 127.9, 128.0, 132.0, 132.1, 134.4, 137.4, 141.4, 142.3, 142.7, 147.7, 148.9, 149.8, 151.0, 151.1, 157.4, 157.9, 158.6, 160.4, 161.3, 162.0, 163.2, 163.7, 176.3, 188.3, 189.1. LCMS (ES+) $m / z=1128.0\left([\mathrm{M}+\mathrm{H}]^{+}, t_{\mathrm{R}}=1.87 \mathrm{~min}\right)$. HRMS (ESI+) $m / z=1127.4014[\mathrm{M}+\mathrm{H}]^{+}$found, $\mathrm{C}_{62} \mathrm{H}_{59} \mathrm{O}_{15} \mathrm{~N}_{6}{ }^{+}$ required 1127.4033 .

(Z)-3-((1-(2-(4-((6-Methoxy-3-oxobenzofuran-2(3H)-ylidene)methyl)phenoxy)ethyl)-1H-1,2,3-triazol-4-yl)met hoxy)-2-(4-(2-(4-((4-oxo-2-phenyl-4H-chr omen-3-yl)oxy)methyl)-1H-1,2,3-triazol-1-yl)ethoxy)phenyl)-4Hchromen-4-one (139). A mixture of alkyne biflavonoid $134(134 \mathrm{mg}, 0.210 \mathrm{mmol})$, azide aurone 89 ( $83.5 \mathrm{mg}, 0.248 \mathrm{mmol}), \mathrm{CuSO}_{4} \cdot 5 \mathrm{H}_{2} \mathrm{O}(70.8 \mathrm{mg}, 0.284 \mathrm{mmol})$ and sodium ascorbate $(131 \mathrm{mg}, 0.659 \mathrm{mmol})$ in $t-\mathrm{BuOH} / \mathrm{H}_{2} \mathrm{O}(1: 1,40 \mathrm{~mL})$ was reacted according to GP-A. The crude residue was purified by flash column chromatography $\left(\mathrm{SiO}_{2}, 1 \%-5 \% \mathrm{MeOH} / \mathrm{CH}_{2} \mathrm{Cl}_{2}\right)$ to afford triazole hybrid $139(73.9 \mathrm{mg}$, $36 \%)$ as a bright yellow powdery solid. m.p. $98-100{ }^{\circ} \mathrm{C}$. TLC $R_{\mathrm{f}}=0.39\left(7 \% \mathrm{MeOH} / \mathrm{CH}_{2} \mathrm{Cl}_{2}\right)$. IR $v_{\max }$ (neat) $/ \mathrm{cm}^{-1}$ : 3001w (C-H str), 2929w (C-H str), 2874w (C-H str), 1693m (C=O str), 1632m, 1600s (C=C str), 1509m (C=C str), 1466m, 1444w, 1395m, 1343w, 1248s, 1181s, 1146m, 1129m, 1110m, 1096w, 1044m. ${ }^{1} \mathrm{H}-\mathrm{NMR}\left(500 \mathrm{MHz}, \mathrm{CDCl}_{3}\right): \delta 3.93\left(3 \mathrm{H}, \mathrm{s},-\mathrm{OCH}_{3}\right), 4.34-4.37\left(4 \mathrm{H}, \mathrm{m}, 2 \times-\mathrm{OCH}_{2} \mathrm{CH}_{2} \mathrm{~N}-\right), 4.69-4.72$ $\left(4 \mathrm{H}, \mathrm{m}, 2 \times-\mathrm{OCH}_{2} \mathrm{CH}_{2} \mathrm{~N}-\right), 5.32\left(4 \mathrm{H}, \mathrm{s}, 2 \times-\mathrm{OCH}_{2} \mathrm{CN}-\right), 6.77(1 \mathrm{H}, \mathrm{d}, J=5.6 \mathrm{~Hz}, \mathrm{ArH}), 6.77(1 \mathrm{H}, \mathrm{s}$, $-\mathrm{C}=\mathrm{CH}), 6.88(2 \mathrm{H}, \mathrm{d}, J=8.8 \mathrm{~Hz}, \mathrm{ArH}), 6.90(2 \mathrm{H}, \mathrm{d}, J=8.8 \mathrm{~Hz}, \mathrm{ArH}), 7.40-7.45(6 \mathrm{H}, \mathrm{m}, \mathrm{ArH}), 7.51(1 \mathrm{H}$, $\mathrm{d}, J=3.6 \mathrm{~Hz}, \mathrm{ArH}), 7.53(1 \mathrm{H}, \mathrm{d}, J=3.6 \mathrm{~Hz}, \mathrm{ArH}), 7.67-7.72(3 \mathrm{H}, \mathrm{m}, \mathrm{ArH}), 7.81(2 \mathrm{H}, \mathrm{d}, J=9.2 \mathrm{~Hz}$, $\mathrm{ArH}), 7.84(1 \mathrm{H}, \mathrm{s},-\mathrm{CHN}-), 7.88(1 \mathrm{H}, \mathrm{s},-\mathrm{CHN}-), 8.01-8.05(4 \mathrm{H}, \mathrm{m}, \mathrm{ArH}), 8.27(2 \mathrm{H}, \mathrm{dd}, J=8.0,1.6 \mathrm{~Hz}$, ArH). ${ }^{13} \mathrm{C}-\mathrm{NMR}\left(500 \mathrm{MHz}, \mathrm{CDCl}_{3}\right): \delta 49.4,49.5,56.0,64.9,65.2,66.2,66.3,96.6,111.5,112.1,114.3$, 114.9, 115.0, 118.0, 118.1, 124.0, 124.1, 124.1, 124.8, 124.8, 124.9, 125.0, 125.7, 125.7, 125.7, 126.1, 128.4, 128.7, 130.6, 130.7, 133.1, 133.4, 133.6, 139.0, 139.6, 144.0, 144.1, 147.0, 155.2, 155.3, 156.0, 156.4, 158.8, $159.5,167.3,168.3,175.0,175.1,182.8$. LCMS (ES+) $m / z=975.4\left([\mathrm{M}+\mathrm{H}]^{+}, t_{\mathrm{R}}=4.94 \mathrm{~min}\right)$. HRMS (ESI +$)$ $m / z=975.2956[\mathrm{M}+\mathrm{H}]^{+}$found, $\mathrm{C}_{56} \mathrm{H}_{43} \mathrm{O}_{11} \mathrm{~N}_{6}{ }^{+}$required 975.2984.

2-(4-(2-(4-((4-((E)-3-(Ferrocenyl)acryloyl)-2-methoxyphenoxy)methyl)-1H-1,2,3-triazol-1-yl)ethoxy)phenyl)-3((1-(2-(4-(((Z)-6-methoxy-3-oxobenzofuran-2(3H)-ylidene)methyl)phenoxy)ethyl)-1H-1,2,3-triazol-4-yl)metho xy)-4H-chromen-4-one (140). A mixture of alkyne biflavonoid 135 (71.6 mg, $0.0940 \mathrm{mmol})$, azide aurone 89 (38.1 mg, $0.113 \mathrm{mmol}), \mathrm{CuSO}_{4} \cdot 5 \mathrm{H}_{2} \mathrm{O}(46.1 \mathrm{mg}, 0.185 \mathrm{mmol})$ and sodium ascorbate $(58.0 \mathrm{mg}$, $0.293 \mathrm{mmol})$ in $t-\mathrm{BuOH} / \mathrm{H}_{2} \mathrm{O}(1: 1,40 \mathrm{~mL})$ was reacted according to GP-A. The crude residue was purified by flash column chromatography $\left(\mathrm{SiO}_{2}, 1 \%-5 \% \mathrm{MeOH} / \mathrm{CH}_{2} \mathrm{Cl}_{2}\right)$ and further purified by preparative HPLC to afford triazole hybrid $140(2.80 \mathrm{mg}, 3 \%)$ as a bright red powdery solid. m.p. $158-160{ }^{\circ} \mathrm{C}$. TLC $R_{\mathrm{f}}=0.35\left(7 \% \mathrm{MeOH} / \mathrm{CH}_{2} \mathrm{Cl}_{2}\right)$. IR $v_{\max }$ (neat) $/ \mathrm{cm}^{-1}: 2917 \mathrm{w}(\mathrm{C}-\mathrm{H} \mathrm{str}), 2852 \mathrm{w}$ (C-H str), 1737w, 1605s (C=O str), 1509m (C=C str), 1465m, 1443w, 1378w, 1353w, 1260s, 1198m, 1151m, 1133w, 1113w, 1042w, 1015w. ${ }^{1} \mathrm{H}-\mathrm{NMR}\left(500 \mathrm{MHz}, \mathrm{CDCl}_{3}\right): \delta 3.94\left(3 \mathrm{H}, \mathrm{s},-\mathrm{OCH}_{3}\right), 3.96\left(3 \mathrm{H}, \mathrm{s},-\mathrm{OCH}_{3}\right)$, $4.17\left(5 \mathrm{H}\right.$, br s, $\left.-\mathrm{C}_{5} \mathrm{H}_{5}\right), 4.38\left(2 \mathrm{H}, \mathrm{t}, J=5.0 \mathrm{~Hz},-\mathrm{OCH}_{2} \mathrm{CH}_{2} \mathrm{~N}-\right), 4.42\left(2 \mathrm{H}, \mathrm{t}, J=5.0 \mathrm{~Hz},-\mathrm{OCH}_{2} \mathrm{CH}_{2} \mathrm{~N}-\right), 4.47$ 
$\left(2 \mathrm{H}\right.$, br s, $\left.-\mathrm{C}_{5} \mathrm{H}_{4}\right), 4.57\left(2 \mathrm{H}\right.$, br s, $\left.-\mathrm{C}_{5} \mathrm{H}_{4}\right), 4.73\left(2 \mathrm{H}, \mathrm{t}, \mathrm{J}=5.0 \mathrm{~Hz},-\mathrm{OCH}_{2} \mathrm{CH}_{2} \mathrm{~N}-\right), 4.80(2 \mathrm{H}, \mathrm{t}, J=4.5 \mathrm{~Hz}$, $\left.-\mathrm{OCH}_{2} \mathrm{CH}_{2} \mathrm{~N}-\right)$, 5.30 (2H, s, $\left.-\mathrm{OCH}_{2} \mathrm{CN}-\right), 5.41\left(2 \mathrm{H}, \mathrm{s},-\mathrm{OCH}_{2} \mathrm{CN}-\right)$, 6.76-6.79 (2H, m, ArH and $\left.-\mathrm{C}=\mathrm{CH}\right)$, $6.77(1 \mathrm{H}, \mathrm{d}, J=14.5 \mathrm{~Hz},-\mathrm{CH}=\mathrm{CHCO}-), 6.89(2 \mathrm{H}, \mathrm{d}, J=9.0 \mathrm{~Hz}, \mathrm{ArH}), 6.90(2 \mathrm{H}, \mathrm{d}, J=8.5 \mathrm{~Hz}, \mathrm{ArH})$, $7.14(1 \mathrm{H}, \mathrm{d}, J=8.0 \mathrm{~Hz}, \mathrm{ArH}), 7.43(1 \mathrm{H}, \mathrm{t}, J=8.0 \mathrm{~Hz}, \mathrm{ArH}), 7.53(1 \mathrm{H}, \mathrm{d}, J=8.5 \mathrm{~Hz}, \mathrm{ArH}), 7.58(1 \mathrm{H}, \mathrm{d}$, $J=8.2 \mathrm{~Hz}, \mathrm{ArH}), 7.62(1 \mathrm{H}, \mathrm{s}, \mathrm{ArH}), 7.70(1 \mathrm{H}, \mathrm{t}, J=8.5 \mathrm{~Hz}, \mathrm{ArH}), 7.70(1 \mathrm{H}, \mathrm{d}, J=16.0 \mathrm{~Hz},-\mathrm{CH}=\mathrm{CHCO}-)$, $7.71(2 \mathrm{H}, \mathrm{d}, J=8.5 \mathrm{~Hz}, \mathrm{ArH}), 7.84(2 \mathrm{H}, \mathrm{d}, J=9.0 \mathrm{~Hz}, \mathrm{ArH}), 7.89(1 \mathrm{H}, \mathrm{s},-\mathrm{CHN}-), 7.94(1 \mathrm{H}, \mathrm{s},-\mathrm{CHN}-)$, $8.05(2 \mathrm{H}, \mathrm{d}, J=9.0 \mathrm{~Hz}, \mathrm{ArH}), 8.27(1 \mathrm{H}, \mathrm{dd}, J=8.0,1.5 \mathrm{~Hz}, \mathrm{ArH}) .{ }^{13} \mathrm{C}-\mathrm{NMR}\left(500 \mathrm{MHz}, \mathrm{CDCl}_{3}\right): \delta 49.6$, $49.7,56.1,61.2,62.8,66.2,66.3,68.9,69.8,71.3,79.3,96.6,111.2,111.6,111.8,112.1,112.3,114.3,114.3$, $114.9,118.0,118.6,121.3,122.5,122.5,124.1,124.1,124.3,124.4,124.8,125.1,125.7,125.7,125.8,130.6$, 133.0, 133.2, 133.5, 138.3, 144.0, 146.0, 149.6, 151.4, 155.1, 157.4, 158.8, 159.5, 167.5, 167.7, 179.2, 182.9, 187.9. LCMS (ES+) $m / z=1098.8\left([\mathrm{M}+\mathrm{H}]^{+}, t_{\mathrm{R}}=2.02 \mathrm{~min}\right)$. HRMS (ESI+) $m / z=1099.2960[\mathrm{M}+\mathrm{H}]^{+}$ found, $\mathrm{C}_{61} \mathrm{H}_{51} \mathrm{FeN}_{6} \mathrm{O}_{11}{ }^{+}$required 1099.2921.

\subsection{Biological Screening}

\subsubsection{A $\beta$ Preparation}

$\mathrm{A} \beta_{42}(1 \mathrm{mg})$ was purchased from Eurogentec Ltd. (Hampshire, UK) as a lyophilised powder. The peptide was dissolved in trifluroacetic acid (TFA, $1 \mathrm{~mL}$ ), sonicated in an ice-water bath for $60 \mathrm{~s}$, then the TFA removed in a vacuum desiccator. Ice cold 1,1,1,3,3,3-hexafluro-2-propanol (HFIP, $1 \mathrm{~mL}$ ) was added to re-suspend the lyophilised peptide. The sample was sonicated for $60 \mathrm{~s}$ at $0{ }^{\circ} \mathrm{C}$, then aliquoted into $20 \mu \mathrm{L}$ portions. The HFIP was removed in the vacuum desiccator overnight and the lyophilised samples were stored at $-80{ }^{\circ} \mathrm{C}$ until use. The required concentration of $A \beta_{42}$ was prepared by dissolving the sample in dimethyl sulfoxide (DMSO) (5\% of total solvent volume), then adding sodium phosphate buffer $\left(50 \mathrm{mM}\right.$, pH 7.4). The solution was sonicated at $0{ }^{\circ} \mathrm{C}$ for 3 min, then centrifuged at $13,400 \mathrm{rpm}$ for $30 \mathrm{~min}$ at $0^{\circ} \mathrm{C}$ to separate any aggregated species.

\subsubsection{Thioflavin T (THT) Assay}

ThT was purchased from AbCam (Cambridge, UK). Final concentrations of $10 \mu \mathrm{M} \mathrm{A} \beta_{42}, 20 \mu \mathrm{M}$ ThT and $50 \mu \mathrm{M}$ compound in sodium phosphate buffer $(50 \mathrm{mM}, \mathrm{pH} 7.4)$ were used for all samples. The assay samples $(100 \mu \mathrm{L})$ were mixed in a black non-binding 96-well plate (Greiner Bio-One, Stonehouse, UK) which was sealed (Nunc ${ }^{\mathrm{TM}}$ polyolefin acrylate film Nunc, ThermoFisher) and loaded into the fluorescence plate reader (Tecan, Männedorf, Switzerland) at $37^{\circ} \mathrm{C}$. Fluorescence kinetics were measured at 5 min reading intervals, with 15 s shaking before each read. The excitation and emission wavelengths were 440 and $480 \mathrm{~nm}$ respectively.

\section{Conclusions}

Herein, we have described a highly modular branching-type strategy for the synthesis of biologically interesting and rare triazole-linked flavonoid dimers and trimers by the varied combination of readily-accessible flavonoid building blocks. Application of this strategy enabled concise and highly step-efficient access to a structurally diverse library of 46 final compounds, with six different biologically-relevant flavonoid structural subclasses (chalcone, flavonol, aurone, flavone, coumarin and isoflavone) successfully incorporated into the library. Each library member features structural motifs that are associated with biological activity (at least two flavonoid units and a 1,2,3-triazole linkage) and many also incorporate additional potential biomolecular-interacting elements (for example, hydrogen-bonding motifs). Many library compounds also feature groups that could provide synthetic handles for further elaboration or diversification. The synthetic strategy could conceivably be applied on a larger scale using a greater range of building blocks. However, this current strategy is limited to the installation of one linker type between the flavonoid units. It may be possible to adapt the strategy to allow for greater variation in the linker motif (for example, the use of an alternate type of building block may allow the alkyl chain length to be varied and 1,5-triazole linkages could conceivably be accessed 
though ruthenium-mediated 'click' cycloaddition conditions). Such variety may be of value in the context of biological screening; for example, previous studies of flavonoid dimers have suggested that linker length variation had a significant effect upon biological activity [14,16]. Preliminary biological screening of a representative sub-set of compounds has revealed that a selection of the triazole-linked dimers exhibit moderate inhibitory activity against the aggregation of $A \beta_{42}$, a process closely linked with the development of Alzheimer's disease. Such findings prompt for continued screening of the entire library and further study of the active scaffolds identified. Milligram (typically multimilligram) quantities of most final library compounds were isolated, which should provide ample material for screening in a wider range of biological assays; the systematic modification of any compounds with interesting properties should be facilitated by the conciseness and inherent modularity of the synthetic strategy [55]. More detailed biological assessment of the compound library is currently ongoing and notable results will be reported in due course.

Supplementary Materials: Supplementary materials can be accessed at: http://www.mdpi.com/1420-3049/21/ 9/1230/s1.

Acknowledgments: We thank the Cambridge Commonwealth Trust for the awards of scholarships to T.H.S. and T.J.S. The research leading to these results has received funding from the European Research Council under the European Union's Seventh Framework Programme (FP7/2007-2013)/ERC grant agreement No. [279337/DOS]. The authors also thank AstraZeneca, the European Union (EU), the Engineering and Physical Sciences Research Council (EPSRC), the Biotechnology and Biological Sciences Research Council (BBSRC), the Medical Research Council (MRC), and the Wellcome Trust for funding. Data accessibility: all data supporting this study are included in the paper and provided as Supporting Information accompanying this paper.

Author Contributions: D.R.S., T.H.S. and T.J.S. conceived and designed the synthetic experiments; F.H. and S.C. conceived and designed the biological experiments, T.H.S. and T.J.S. performed the synthetic experiments; S.C. performed the biological experiments; T.H.S. and T.J.S. analyzed the chemical data; D.R.S. supervised the project; T.H.S., T.J.S., W.R.J.D.G. and D.G.T. co-wrote the manuscript.

Conflicts of Interest: The authors declare no conflict of interest. The founding sponsors had no role in the design of the study; in the collection, analyses, or interpretation of data; in the writing of the manuscript, and in the decision to publish the results.

\section{References}

1. Sum, T.H.; Sum, T.J.; Stokes, J.E.; Galloway, W.R.J.D.; Spring, D.R. Divergent and concise total syntheses of dihydrochalcones and 5-deoxyflavones recently isolated from Tacca species and Mimosa diplotricha. Tetrahedron 2015, 71, 4557-4564. [CrossRef]

2. Wu, B.; Zhang, W.; Li, Z.; Gu, L.; Wang, X.; Wang, P.G. Concise synthesis of 5-methoxy-6-hydroxy-2methylchromone-7-O- and 5-hydroxy-2-methylchromone-7-O-rutinosides. Investigation of their cytotoxic activities against several human tumor cell lines. J. Org. Chem. 2011, 76, 2265-2268. [CrossRef] [PubMed]

3. Briot, A.; Baehr, C.; Brouillard, R.; Wagner, A.; Mioskowski, C. Concise synthesis of dihydrochalcones via palladium-catalyzed coupling of aryl halides and 1-aryl-2-propen-1-ols. J. Org. Chem. 2004, 69, 1374-1377. [CrossRef] [PubMed]

4. Silva, D.H.; Zhang, Y.; Santos, L.A.; Bolzani, V.S.; Nair, M.G. Lipoperoxidation and cyclooxygenases 1 and 2 inhibitory compounds from Iryanthera juruensis. J. Agric. Food Chem. 2007, 55, 2569-2574. [CrossRef] [PubMed]

5. Snijman, P.W.; Joubert, E.; Ferreira, D.; Li, X.C.; Ding, Y.; Green, I.R.; Gelderblom, W.C. Antioxidant activity of the dihydrochalcones Aspalathin and Nothofagin and their corresponding flavones in relation to other Rooibos (Aspalathus linearis) Flavonoids, Epigallocatechin Gallate, and Trolox. J. Agric. Food Chem. 2009, 57, 6678-6684. [CrossRef] [PubMed]

6. Hermoso, A.; Jimenez, I.A.; Mamani, Z.A.; Bazzocchi, I.L.; Pinero, J.E.; Ravelo, A.G.; Valladares, B. Antileishmanial activities of dihydrochalcones from piper elongatum and synthetic related compounds. Structural requirements for activity. Bioorg. Med. Chem. 2003, 11, 3975-3980. [CrossRef]

7. Sum, T.J.; Sum, T.H.; Galloway, W.R. J.D.; Spring, D.R. Divergent total syntheses of flavonoid natural products isolated from Rosa rugosa and Citrus unshiu. Synlett 2016, 27, 1725-1727. 
8. Meguellati, A.; Ahmed-Belkacem, A.; Nurisso, A.; Yi, W.; Brillet, R.; Berqouch, N.; Chavoutier, L.; Fortune, A.; Pawlotsky, J.-M.; Boumendjel, A.; et al. New pseudodimeric aurones as palm pocket inhibitors of Hepatitis C virus RNA-dependent RNA polymerase. Eur. J. Med. Chem. 2016, 115, 217-229. [CrossRef] [PubMed]

9. Sashidhara, K.V.; Kumar, A.; Kumar, M.; Sarkar, J.; Sinha, S. Synthesis and in vitro evaluation of novel coumarin-chalcone hybrids as potential anticancer agents. Bioorg. Med. Chem. Lett. 2010, 20, 7205-7211. [CrossRef] [PubMed]

10. Chow, L.M.C.; Chan, T.; Chan, K.F.; Wong, I.L.K.; Man, C. Preparation of Alkyne-, Azide- and Triazole-Containing Flavonoids as Modulators for Multidrug Resistance in Cancer. WO 2013127361 A1, 6 September 2013.

11. Pingaew, R.; Saekee, A.; Mandi, P.; Nantasenamat, C.; Prachayasittikul, S.; Ruchirawat, S.; Prachayasittikul, V. Synthesis, biological evaluation and molecular docking of novel chalcone-coumarin hybrids as anticancer and antimalarial agents. Eur. J. Med. Chem. 2014, 85, 65-76. [CrossRef] [PubMed]

12. Yan, C.S.; Wong, I.L.; Chan, K.F.; Kan, J.W.; Chong, T.C.; Law, M.C.; Zhao, Y.; Chan, S.W.; Chan, T.H.; Chow, L.M. A new class of safe, potent, and specific P-gp modulator: Flavonoid dimer FD18 reverses P-gp-mediated multidrug resistance in human breast xenograft in vivo. Mol. Pharm. 2015, 12, 3507-3517. [CrossRef] [PubMed]

13. Chan, T.-H.; Chow, L.M.-C. Preparation of Flavonoid Dimers for Reducing P-glycoprotein Based Multidrug Resistance. WO 2007135592 A1, 29 November 2007.

14. Wong, I.L.; Chan, K.F.; Chen, Y.F.; Lun, Z.R.; Chan, T.H.; Chow, L.M. In vitro and in vivo efficacy of novel flavonoid dimers against cutaneous leishmaniasis. Antimicrob. Agents Chemother. 2014, 58, 3379-3388. [CrossRef] [PubMed]

15. Chan, K.F.; Wong, I.L.; Kan, J.W.; Yan, C.S.; Chow, L.M.; Chan, T.H. Amine linked flavonoid dimers as modulators for P-glycoprotein-based multidrug resistance: Structure-activity relationship and mechanism of modulation. J. Med. Chem. 2012, 55, 1999-2014. [CrossRef] [PubMed]

16. Wong, I.L.K.; Chan, K.-F.; Burkett, B.A.; Zhao, Y.; Chai, Y.; Sun, H.; Chan, T.H.; Chow, L.M.C. Flavonoid dimers as bivalent modulators for pentamidine and sodium stiboglucanate resistance in leishmania. Antimirob. Agents Chemother. 2007, 51, 930-940. [CrossRef] [PubMed]

17. Chan, K.-F.; Zhao, Y.; Chow, T.W.S.; Yan, C.S.W.; Ma, D.L.; Burkett, B.A.; Wong, I.L.K.; Chow, L.M.C.; Chan, T.H. Flavonoid dimers as bivalent modulators for P-glycoprotein-based multidrug resistance: Structure-activity relationships. ChemMedChem 2009, 4, 594-614. [CrossRef] [PubMed]

18. Hou, J.; Liu, X.; Shen, J.; Zhao, G.; Wang, P.G. The impact of click chemistry in medicinal chemistry. Expert Opin. Drug Discov. 2012, 7, 489-501. [CrossRef] [PubMed]

19. Fujii, H.; Watanabe, A.; Nemoto, T.; Narita, M.; Miyoshi, K.; Nakamura, A.; Suzuki, T.; Nagase, H. Synthesis of novel twin drug consisting of 8-oxaendoethanotetrahydromorphides with a 1,4-dioxane spacer and its pharmacological activities: $\mu, \kappa$, and putative $\varepsilon$ opioid receptor antagonists. Bioorg. Med. Chem. Lett. 2009, 19, 438-441. [CrossRef] [PubMed]

20. Njogu, P.M.; Gut, J.; Rosenthal, P.J.; Chibale, K. Design, synthesis, and antiplasmodial activity of hybrid compounds based on (2R,3S)-N-benzoyl-3-phenylisoserine. ACS Med. Chem. Lett. 2013, 4, 637-641. [CrossRef] [PubMed]

21. Hein, J.E.; Fokin, V.V. Copper-catalyzed azide-alkyne cycloaddition (CuAAC) and beyond: New reactivity of copper(I) acetylides. Chem. Soc. Rev. 2010, 39, 1302-1315. [CrossRef]

22. Zhang, J.; Fu, X.-L.; Yang, N.; Wang, Q.-A. Synthesis and cytotoxicity of chalcones and 5-deoxyflavonoids. Sci. World J. 2013, 2013, 649485. [CrossRef] [PubMed]

23. Xiong, Y.; Schaus, S.E.; Porco, J.A., Jr. Metal-catalyzed cascade rearrangements of 3-alkynyl flavone ethers. Org. Lett. 2013, 15, 1962-1965. [CrossRef] [PubMed]

24. Liu, J.; Taylor, S.F.; Dupart, P.S.; Arnold, C.L.; Sridhar, J.; Jiang, Q.; Wang, Y.; Skripnikova, E.V.; Zhao, M.; Foroozesh, M. Pyranoflavones: A group of small-molecule probes for exploring the active site cavities of cytochrome P450 enzymes 1A1, 1A2, and 1B1. J. Med. Chem. 2013, 56, 4082-4092. [CrossRef] [PubMed]

25. Yeap, G.-Y.; Yam, W.-S.; Takeuchi, D.; Osakada, K.; Gorecka, E.; Mahmood, W.A.K.; Boey, P.-L.; Hamid, S.A. Synthesis, thermal stabilities, and anisotropic properties of some new isoflavone-based esters 7-decanoyloxy-3-(4'-substitutedphenyl)-4H-1-benzopyran-4-ones. Liq. Cryst. 2008, 35, 315-323. [CrossRef]

26. Beney, C.; Mariotte, A.-M.; Boumendjel, A. An efficient synthesis of 4.6-dimethoxyaurones. Heterocycles 2001, 55, 967-972. 
27. Zheng, Y.C.; Duan, Y.C.; Ma, J.L.; Xu, R.M.; Zi, X.; Lv, W.L.; Wang, M.M.; Ye, X.W.; Zhu, S.; Mobley, D.; et al. Triazole-dithiocarbamate based selective lysine specific demethylase 1 (LSD1) inactivators inhibit gastric cancer cell growth, invasion, and migration. J. Med. Chem. 2013, 56, 8543-8560. [CrossRef] [PubMed]

28. Li, S.Y.; Wang, X.B.; Xie, S.S.; Jiang, N.; Wang, K.D.; Yao, H.Q.; Sun, H.B.; Kong, L.Y. Multifunctional tacrine-flavonoid hybrids with cholinergic, $\beta$-amyloid-reducing, and metal chelating properties for the treatment of Alzheimer's disease. Eur. J. Med. Chem. 2013, 69, 632-646. [CrossRef] [PubMed]

29. Detsi, A.; Majdalani, M.; Kontogiorgis, C.A.; Hadjipavlou-Litina, D.; Kefalas, P. Natural and synthetic 2'-hydroxy-chalcones and aurones: Synthesis, characterization and evaluation of the antioxidant and soybean lipoxygenase inhibitory activity. Bioorg. Med. Chem. 2009, 17, 8073-8085. [CrossRef] [PubMed]

30. Querfurth, H.W.; LaFerla, F.M. Alzheimer's disease. N. Engl. J. Med. 2010, 362, 329-344. [CrossRef] [PubMed]

31. Citron, M. Alzheimer's disease: Strategies for disease modification. Nat. Rev. Drug Discov. 2010, 9, 387-398. [CrossRef] [PubMed]

32. Ehrnhoefer, D.E. EGCG redirects amyloidogenic polypeptides into unstructured, off-pathway oligomers. Nat. Struct. Mol. Biol. 2008, 15, 558-566. [CrossRef] [PubMed]

33. Necula, M.; Kayed, R.; Milton, S.; Glabe, C.G. Small molecule inhibitors of aggregation indicate that amyloid $\beta$ oligomerization and fibrillization pathways are independent and distinct. J. Biol. Chem. 2007, 282, 10311-10324. [CrossRef] [PubMed]

34. Ladiwala, A.R.; Dordick, J.S.; Tessier, P.M. Aromatic small molecules remodel toxic soluble oligomers of amyloid beta through three independent pathways. J. Biol. Chem. 2011, 286, 3209-3218. [CrossRef] [PubMed]

35. Ono, K.; Yoshiike, Y.; Takashima, A.; Hasegawa, K.; Naiki, H.; Yamada, M. Potent anti-amyloidogenic and fibril-destabilizing effects of polyphenols in vitro: Implications for the prevention and therapeutics of Alzheimer's disease. J. Neurochem. 2003, 87, 172-181. [CrossRef] [PubMed]

36. Thapa, A.; Woo, E.-R.; Chi, E.Y.; Sharoar, G.; Jin, H.-G.; Shin, S.Y.; Park, I.-S. Biflavonoids Are Superior to Monoflavonoids in Inhibiting Amyloid-B Toxicity and Fibrillogensesi via Accumulation of Nontoxic Oligomer-like Structures. Biochemistry 2011, 50, 2445-2455. [CrossRef] [PubMed]

37. Zammit, S.C.; Cox, A.J.; Gow, R.M.; Zhang, Y.; Gilbert, R.E.; Krum, H.; Kelly, D.J.; Williams, S.J. Evaluation and optimization of antifibrotic activity of cinnamoyl anthranilates. Bioorg. Med. Chem. Lett. 2009, 19, 7003-7006. [CrossRef] [PubMed]

38. Sagrera, G.; Bertucci, A.; Vazquez, A.; Seoane, G. Synthesis and antifungal activities of natural and synthetic biflavonoids. Bioorg. Med. Chem. 2011, 19, 3060-3073. [CrossRef] [PubMed]

39. Kakade, K.P. Synthesis and characterization of some bromo substituted chalcone by the green synthesis way (grinding method) and aurones 2-benzylidine-1-benzofuran-3-one by cyclization method. World J. Pharm. Pharm. Sci. 2015, 4, 1591-1597.

40. Tiwari, N.T.; Monserrat, J.-P.; de Montigny, F.; Jaouen, G.; Rager, M.-N.; Hillard, E. Synthesis and structural characterization of ferrocenyl-substituted aurones, flavones, and flavonols. Organometallics 2011, 30, 5424-5432. [CrossRef]

41. Hans, R.H.; Guantai, E.M.; Lategan, C.; Smith, P.J.; Wan, B.; Franzblau, S.G.; Gut, J.; Rosenthal, P.J.; Chibale, K. Synthesis, antimalarial and antitubercular activity of acetylenic chalcones. Bioorg. Med. Chem. Lett. 2010, 20, 942-944. [CrossRef] [PubMed]

42. Yadav, D.K.; Gautam, A.K.; Kureel, J.; Srivastava, K.; Sahai, M.; Singh, D.; Chattopadhyay, N.; Maurya, R. Synthetic analogs of daidzein, having more potent osteoblast stimulating effect. Bioorg. Med. Chem. Lett. 2011, 21, 677-681. [CrossRef] [PubMed]

43. Vontzalidou, A.; Zoidis, G.; Chaita, E.; Makropoulou, M.; Aligiannis, N.; Lambrinidis, G.; Mikros, E.; Skaltsounis, A.L. Design, synthesis and molecular simulation studies of dihydrostilbene derivatives as potent tyrosinase inhibitors. Bioorg. Med. Chem. Lett. 2012, 22, 5523-5526. [CrossRef] [PubMed]

44. Yadav, S.K. Process for the preparation of chromones, isoflavones and homoisoflavones using Vilsmeier reagent generated from phthaloyl dichloride and DMF. Int. J. Org. Chem. 2014, 4, 236-246. [CrossRef]

45. Daniel, V.; Rao, Y.J.; Kumar, K.S.; Krupadanam, G.L.D. A facile synthesis of angular and linera 8/2-methyl-furo[2,3- $h] /[3,2-g]$ chromones and angular pyrano[2,3-f] isoflavones from 7-propargyloxy chromones and isoflavones. Heterocycl. Commun. 2008, 14, 337-344. [CrossRef]

46. Rao, Ch.P.; Srimannarayana, G. Claisen rearrangement of 4-propargloxycoumarins: Formation of $2 \mathrm{H}, 5 \mathrm{H}$ pyrano[3,2-c][1]benzopyran-5-ones. Synth. Commun. 1990, 20, 535-540. [CrossRef] 
47. Bolek, D.; Gutschow, M. Preparation of 4,6,3',4'-tetrasubstituted aurones via aluminium oxide-catalyzed condensation. J. Heterocycl. Chem. 2005, 42, 1399-1403. [CrossRef]

48. Haudecoeur, R.; Ahmed-Belkacem, A.; Yi, W.; Fortune, A.; Brillet, R.; Belle, C.; Nicolle, E.; Pallier, C.; Pawlotsky, J.M.; Boumendjel, A. Discovery of naturally occurring aurones that are potent allosteric inhibitors of hepatitis C virus RNA-dependent RNA polymerase. J. Med. Chem. 2011, 54, 5395-5402. [CrossRef] [PubMed]

49. Leonetti, F.; Favia, A.; Rao, A.; Aliano, R.; Paluszcak, A.; Hartmann, R.W.; Carotti, A. Design, synthesis, and 3D QSAR of novel potent and selective aromatase inhibitors. J. Med. Chem. 2004, 47, 6792-6803. [CrossRef] [PubMed]

50. Zhang, C.; Zhao, J.; Wu, S.; Wang, Z.; Wu, W.; Ma, J.; Guo, S.; Huang, L. Intramolecular RET enhanced visible light-absorbing bodipy organic triplet photosensitizers and application in photooxidation and triplet-triplet annihilation upconversion. J. Am. Chem. Soc. 2013, 135, 10566-10578. [CrossRef] [PubMed]

51. Chen, C.Y.; Chen, C.T. A PNIPAM-based fluorescent nanothermometer with ratiometric readout. Chem. Commun. 2011, 47, 994-996. [CrossRef] [PubMed]

52. Rao, N.S.; Kistareddy, C.; Bhavani, B.; Bhavani, R. Synthesis, antibacterial and antifungal activity of some novel chalcone derivatives derived from Apocynin. Chem. J. 2013, 3, 143-148.

53. Senthilkumar, N.; Somannavar, W.S.; Reddy, S.B.; Sinha, B.K.; Narayan, G.K.A.S.S.; Dandala, R.; Mukkanti, K. Synthesis of active metabolites of Carvedilol, an antihypertensive drug. Synth. Commun. 2011, 41, 268-276. [CrossRef]

54. Sridhar, J.; Ellis, J.; Dupart, P.; Liu, J.; Stevens, C.L.; Foroozesh, M. Development of flavone propargyl ethers as potent and selective inhibitors of cytochrome P450 enzymes 1A1 and 1A2. Drug Metab. Lett. 2012, 6, 275-284. [CrossRef] [PubMed]

55. Isidro-Llobet, A.; Hadje Georgiou, K.; Galloway, W.R.J.D.; Giacomini, E.; Hansen, M.R.; Mendez-Abt, G.; Tan, Y.S.; Carro, L.; Sore, H.F.; Spring, D.R. A diversity-oriented synthesis strategy enabling the combinatorial-type variation of macrocyclic peptidomimetic scaffolds. Org. Biomol. Chem. 2015, 13, 4570-4580. [CrossRef] [PubMed]

Sample Availability: Samples of final library compounds are available from the authors.

(c) 2016 by the authors; licensee MDPI, Basel, Switzerland. This article is an open access article distributed under the terms and conditions of the Creative Commons Attribution (CC-BY) license (http://creativecommons.org/licenses/by/4.0/). 